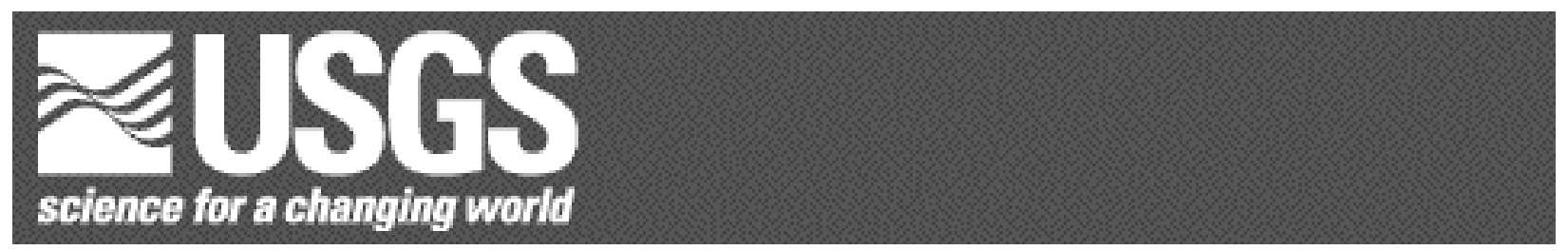

\title{
Conodont and Radiolarian Data from \\ the De Long Mountains Quadrangle and Adjacent Areas, Northern Alaska
}

By Julie A. Dumoulin, ${ }^{1}$ Anita G. Harris, ${ }^{2}$ Charles D. Blome, ${ }^{3}$ and Lorne E. Young 4 Open-File Report 2006-1068

2006

Any use of trade, firm, or product names is for descriptive purposes only and does not imply endorsement by the U.S. Government.

U.S. DEPARTMENT OF THE INTERIOR

U.S. GEOLOGICAL SURVEY

${ }^{1}$ U.S. Geological Survey, 4200 University Drive, Anchorage, AK 99508.

${ }^{2}$ U.S. Geological Survey (retired), 1523 E. Hillsboro Blvd., Apt. 1031, Deerfield Beach, FL 33441.

${ }^{3}$ U.S. Geological Survey, Box 25046, MS 973, Denver, CO 80225.

${ }^{4} 12015$ North Fairwood Drive, Spokane, WA 99218. 


\section{CONTENTS}

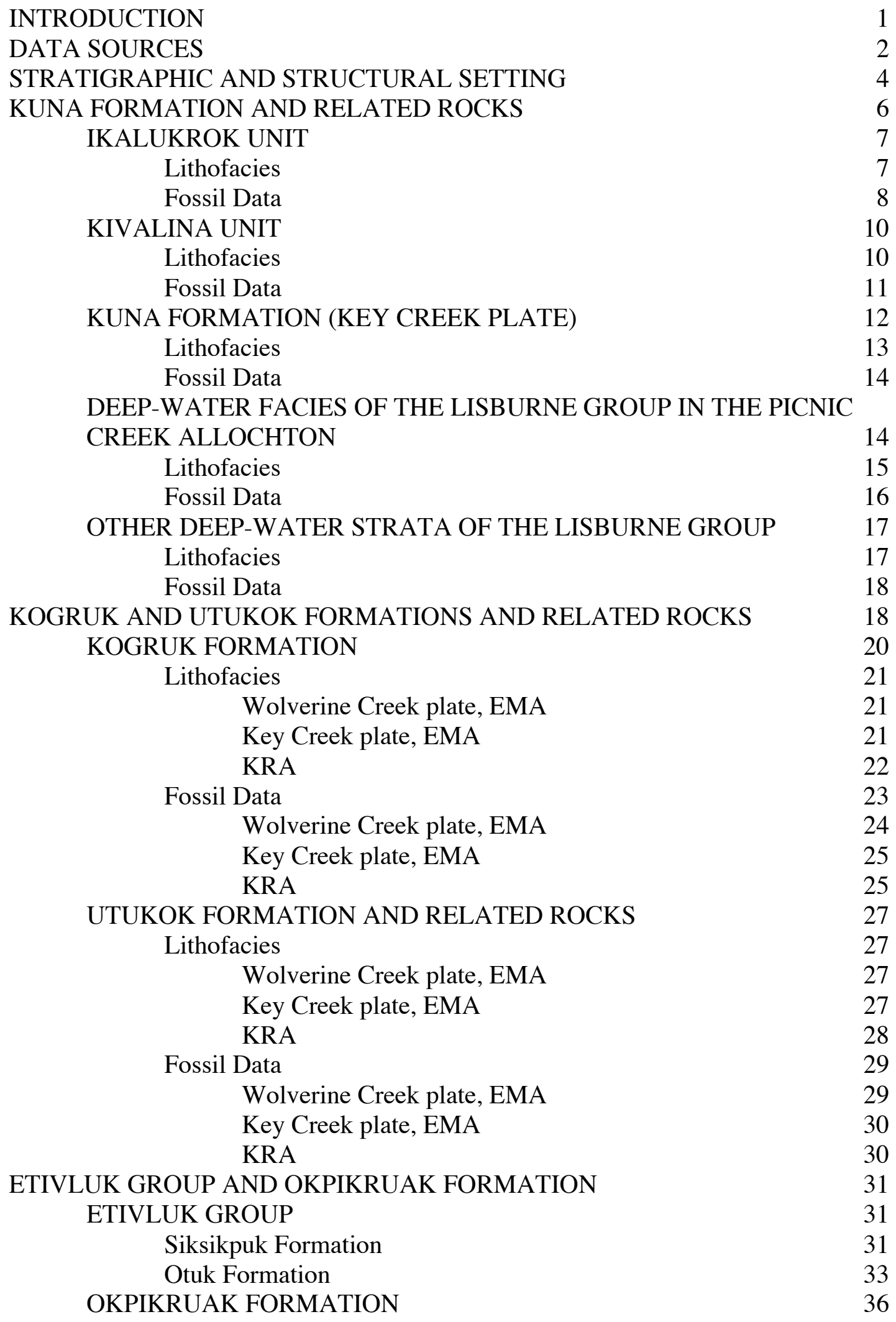


NUKA FORMATION AND RELATED ROCKS

ENDICOTT GROUP 38

DEVONIAN CARBONATE ROCKS $\quad 39$

THERMAL DATA $\quad 42$

ACKNOWLEDGMENTS $\quad 44$

REFERENCES $\quad 44$

FIGURES

Figure 1. Distribution of the Lisburne Group in northern Alaska.

Figure 2. Generalized stratigraphic column for the Endicott Mountains allochthon in the western Brooks Range.

Figure 3. Structural hierarchy for allochthons and plates in the Red Dog area.

Figure 4. Depositional setting, fossil control, and stratigraphic context of Lisburne Group successions in the Red Dog area.

Figure 5. Age range of most biostratigraphically useful conodont and radiolarian collections from deep-water facies of the Lisburne Group.

Figure 6. Age range of most biostratigraphically useful conodont collections from mainly shallow-water facies of the Lisburne Group and Kayak Shale.

Figure 7. Age range of most biostratigraphically useful radiolarian and conodont collections from the Etivluk Group and Nuka Formation.

Figure 8. Age range of most biostratigraphically useful conodont collections from Devonian carbonate rocks.

\section{PLATES}

Plate 1. Conodonts from the Kuna Formation and related deep-water facies of the Lisburne Group.

Plate 2. Conodonts from deep- and shallow-water facies of the Lisburne Group.

Plate 3. Conodonts from various units of the Lisburne Group and from the Kayak Shale of the Endicott Group.

Plate 4. Conodonts from the Lisburne Group, Howard Pass quadrangle.

\section{MAPS}

Map 1. Outcrop fossil localities in the De Long Mountains and Misheguk Mountain quadrangles.

Map 2. Drill hole fossil localities in the Su-Lik and Anarraaq areas.

Map 3. Drill hole fossil localities in the Red Dog Mine area.

Map 4. Outcrop and drill hole fossil localities in the Noatak quadrangle.

\section{TABLES}

Table 1. Conodont samples from the Ikalukrok unit of the Kuna Formation.

Table 2. Conodont samples from the Kivalina unit of the Kuna Formation.

Table 3. Conodont samples from the Kuna Formation.

Table 4. Conodont samples from deep-water facies of the Lisburne Group in the Picnic Creek allochthon.

Table 5. Conodont samples from other deep-water strata of the Lisburne Group.

Table 6. Conodont samples from the Kogruk Formation. 
Table 7. Conodont samples from the Utukok Formation.

Table 8. Conodont samples from unnamed strata that may correlate with the Utukok Formation.

Table 9. Conodont samples from the Etivluk Group and Okpikruak Formation.

Table 10. Conodont samples from the Nuka Formation and related rocks.

Table 11. Conodont samples from the Endicott Group.

Table 12. Conodont samples from Devonian carbonate rocks.

Table 13. Conodont samples from the Noatak quadrangle.

Table 14. Radiolarian samples from the De Long Mountains quadrangle

Table 15. Conodonts illustrated in plates 3 and 4 from the Howard Pass quadrangle. 
Red Dog Fossils OF 2/24/06 4:55 PM p. 1

\title{
CONODONT AND RADIOLARIAN DATA FROM THE DE LONG MOUNTAINS QUADRANGLE AND ADJACENT AREAS, NORTHERN ALASKA
}

\author{
By \\ Julie A. Dumoulin, Anita G. Harris, Charles D. Blome, and Lorne E. Young
}

\section{INTRODUCTION}

This report presents biostratigraphic data from 289 collections at 189 localities in the De Long Mountains, Misheguk Mountain, and Noatak quadrangles (fig. 1); most of these data have never been previously published. The collections were made during studies of the Red Dog massive sulfide deposit in 1998-2004 and in support of regional mapping projects in 1979, 1981, 1983, and 1997-98. The collections-mostly conodonts and some radiolarians - tightly constrain the age of many stratigraphic units of Devonian through Triassic age exposed within the study area, and provide additional data on the depositional environments and thermal history of these rocks.

The data are presented in a series of tables, organized by fossil type, stratigraphic unit, and location. Tables 1-12 contain conodont data, mostly from the De Long Mountains quadrangle. All of these collections were initially examined, or were reevaluated, from 1997 through 2004, and complete faunal lists are given for all samples. Table 13 lists ages and conodont color alteration indices (CAIs) of 27 collections from 24 localities in the Noatak quadrangle; updated faunal lists were not prepared for these samples. Radiolarian data - all from the De Long Mountains quadrangle - are given in table 14; these collections were analyzed between 1998 and 2003. 
Red Dog Fossils OF 2/24/06 4:55 PM p. 2

Collection localities are shown in four maps (sheets 1,2). Map 1 (sheet 1) shows all outcrop samples from the De Long Mountains and western Misheguk Mountain quadrangle (locs. 1-121). Maps 2-4 (sheets 1,2) show all drill hole sample localities; samples come from the Su-Lik deposit and in and around the Anarraaq deposit (map 2, locs. 122-135), in and adjacent to the Red Dog deposits (Paalaaq, Aqqaluk, Main, and Qanaiyaq) (map 3, locs. 136-158), and from drill holes along the Port Road in the Noatak quadrangle (map 4, locs. 159-160). Map 4 (sheet 2) also shows all outcrop samples from the Noatak quadrangle (locs. 161-189).

The text summarizes the lithofacies, age, and biofacies of the Paleozoic and Mesozoic stratigraphic units that produced the fossil collections presented in the tables. Many of the data for the Lisburne Group are taken from Dumoulin and others (2004). Plates 1-4 illustrate important conodonts from the collections listed herein, as well as from coeval collections in the Howard Pass quadrangle; information about the Howard Pass conodonts is given in Table 15.

\section{DATA SOURCES}

The biostratigraphic samples tabulated in this report were collected during a series of investigations spanning 25 years. Irving L. Tailleur and his co-workers Steven M. Curtis, Inyo Ellersieck, and Charles F. Mayfield collected conodont samples during regional mapping of the De Long Mountains quadrangle in 1979 and the Noatak quadrangle in 1981. General ages of some of these collections were given in Curtis and others (1990), Ellersieck and others (1990), and Mayfield and others (1987, 1990), but these ages are herein updated and refined and faunal lists are published for these 
collections for the first time. In 1983, Anita G. Harris collected additional conodont samples from the De Long Mountains area, and Lorne E. Young, Tailleur, and David W. Moore collected a few conodont samples from diamond drill cores of the Su and Red Dog deposits. John S. Kelley and his co-workers Kevin Evans, Jeanine M. Schmidt, and Kirk W. Sherwood collected conodont and radiolarian samples as part of regional mapping and structural investigations in the De Long Mountains quadrangle in 1997 and 1998. Most recently, Julie A. Dumoulin, Anita G. Harris, Karen D. Kelley, and David Leach collected conodont and radiolarian samples from outcrops and drill holes in the De Long Mountains, Misheguk Mountain, and Noatak quadrangles in support of ore deposit studies from 1998 through 2004. Faunal lists from the 1997-2004 collections have never been previously published, although age and biofacies data from some of these localities were summarized in Dumoulin and others (2004).

Other fossil data from the study area are relatively sparse. Megafossil and microfossil data from the De Long Mountains, western Misheguk Mountain, and western Noatak quadrangles are summarized in Curtis and others (1984, 1990), Ellersieck and others (1990), and Mayfield and others (1987, 1990). Radiolarian collections made prior to 1984 are listed in Murchey and others (1988), and Blome and others (1988). Fossil collections (chiefly megafossils listed in unpublished U.S. Geological Survey fossil reports) can be found online in the Alaska Paleontological database at http://alaskafossil.org/.

All tables in this report include lithologic descriptions of the rocks that contained the fossils where these data were available. Petrographic descriptions based on thin section examinations of the sampled lithologies (by J.A. Dumoulin) are also provided for 
most of the collections. Structural level of these collections (allochthon, plate, and locally, subplate) is given where known; most of these determinations were made by L.E. Young and may differ from those previously published by Tailleur and his co-workers. Conodont data tables 1-12 give biofacies and conodont color alteration index (CAI) data wherever these could be determined. These tables also include weights of most of the samples processed for conodonts and any unusual features noted in the heavy-mineral residues. All barren conodont samples collected from 1997 through 2004 are also included in these tables in order to demonstrate the relative productivity of various stratigraphic units. Locations of all fossil collections presented here were verified using original field maps and notes of the geologists that collected them; the verified points were then digitized and latitudes and longitudes of the locations were generated.

\section{STRATIGRAPHIC AND STRUCTURAL SETTING}

Paleozoic and Mesozoic strata of the western Brooks Range are characterized by both stratigraphic and structural complexity; a general stratigraphic column for this area is shown in figure 2. The Kuna Formation of the Lisburne Group hosts all massive sulfide deposits in the Red Dog district, so the Lisburne Group was the chief focus of the sampling reported here. The Lisburne is a chiefly Carboniferous succession that includes both shallow- and deep-water facies and is widely distributed in outcrop and the subsurface of northern Alaska (fig. 1). It is generally underlain by shallow-marine to non-marine siliciclastic rocks of the Devonian-Mississippian Endicott Group. Devonian carbonate rocks (called Baird Group by some previous workers) underlie the Endicott in some areas, but elsewhere directly underlie the Lisburne. Deep-water shale, chert, and 
Red Dog Fossils OF 2/24/06 4:55 PM p. 5

minor carbonate of the Pennsylvanian-Jurassic Etivluk Group (which includes the Siksikpuk and Otuk Formations) overlie the Lisburne and are in turn overlain by JurassicCretaceous flysch of the Okpikruak Formation.

In the western Brooks Range, Paleozoic and Mesozoic strata are exposed in a series of discrete structural allochthons that are in turn made up of plates, subplates, and duplexes (fig. 3). Many nomenclatural schemes have been proposed to encompass the structural and stratigraphic complexity of these strata; in this report, we use the terminology of Young (2004). The Kuna Formation is mainly confined to the Endicott Mountains allochthon (EMA; Brooks Range allochthon of some authors). Deep-water facies of the Lisburne Group that are similar in age and lithology to the Kuna Formation also occur in the structurally higher Picnic Creek (PCA) and Ipnavik River (IRA) allochthons. Coeval shallow-water Lisburne facies (e.g., Utukok and Kogruk Formations) are found mostly in parts of the Endicott Mountains allochthon and in the structurally higher Kelly River allochthon (KRA). A fifth allochthon, the Nuka Ridge (NRA), contains carbonate-rich strata (Nuka Formation) that are coeval with, but are not included in, the Lisburne Group.

The disparate facies of the Lisburne Group outlined above are parts of a single depositional system that has been structurally dismembered; Mayfield et al. (1988) and Young (2004) have proposed the most detailed palinspastic restorations of this system. In this paper, we use the reconstruction of Young (2004), who suggested that deep-water facies of the Lisburne Group in the Red Dog area were deposited in an extensional basin roughly $200 \mathrm{~km}$ wide by $600 \mathrm{~km}$ long that was flanked on the north and southwest by carbonate platforms. 
Conodont collections from the Kuna Formation and related deep-water facies of the Lisburne Group are in tables 1-5. Tables 6-8 contain collections from chiefly shallow-water strata of the Lisburne Group (Utukok and Kogruk Formations and related rocks). Samples from younger strata (Etivluk Group and Okpikruak Formation) are in table 9. Nuka Formation samples are in table 10. Collections from older strata are found in table 11 (Endicott Group) and table 12 (Devonian carbonate rocks). Table 13 contains additional conodont samples from the Lisburne Group, Nuka Formation, Endicott Group, and Devonian carbonate rocks. Radiolarian samples, all from the Kuna Formation and related rocks and the Etivluk Group, are in table 14.

In the following sections, we briefly discuss the lithofacies, age, and biofacies of the Devonian through Triassic strata that were sampled for this report. For a more detailed description of the lithologies and depositional setting of the Lisburne Group, see Dumoulin and others (2004). For a synthesis of regional tectonics and paleogeography, see Young (2004).

\section{KUNA FORMATION AND RELATED ROCKS}

Deep-water facies of the Kuna Formation comprise the entire Lisburne Group in the Red Dog plate and in much of the Key Creek plate of the Endicott Mountains allochthon. In the Red Dog plate, the Kuna Formation is subdivided into informal lower and upper units called the Kivalina and Ikalukrok, respectively (Moore et al., 1986), but subdivisions of the Kuna are not recognized in the Key Creek plate. Deep-water facies also comprise most or all of the Lisburne Group in the Picnic Creek and Ipnavik River 
allochthons. These strata are not generally included in the Kuna Formation and have no formal names.

\section{IKALUKROK UNIT (TABLES 1, 14; PLATE 1)}

\section{Lithofacies}

The Ikalukrok unit makes up the upper part of the Kuna Formation in the Red Dog plate and hosts all massive sulfide deposits in the Red Dog district. It consists mainly of black shale and mudstone, with locally abundant intercalations of carbonate and barite, and greatly subordinate intervals of mainly felsic volcanic rocks. The thickness of the Ikalukrok unit ranges from $<30$ to $>240 \mathrm{~m}$. Much of the variation in total thickness reflects variation in the amount of interbedded carbonate.

Thin-bedded to fissile shale comprises most of the lower part of the Ikalukrok unit, and thicker bedded blocky mudstone and chert predominate in the upper beds. Some chert and siliceous mudstone contain abundant radiolarians and (or) siliceous sponge spicules and probably originated as biosiliceous oozes.

Two main types of carbonate layers, calcareous radiolarite and less abundant lithic turbidite, occur in the Ikalukrok unit. Calcareous radiolarite consists of rare to very abundant radiolarians in a carbonate matrix. Lithic turbidite consists of $<5$ to $>50$ percent carbonate, 5 to 30 percent detrital quartz, and 20 to $>50$ percent other noncarbonate material, including mud, lithic clasts, feldspar, mica, chlorite, and phosphatic grains. We interpret most carbonate layers in the Ikalukrok as mass flow deposits, although some thick sections of calcareous radiolarite could have formed as in situ slope or slope-basin deposits. 
Red Dog Fossils OF 2/24/06 4:55 PM p. 8

The top of the Ikalukrok unit is generally marked by a shift from black to gray chert. In many sections, this color change coincides with a horizon of mammiform structures that can reach $75 \mathrm{~cm}$ in maximum horizontal dimension, exceed $1 \mathrm{~m}$ in height, and may represent fluid- and/or gas-escape conduits (Young, 2004).

The internal stratigraphy of the Ikalukrok unit has been studied in detail in the Anarraaq deposit (Kelley and others, 2004). Three subdivisions of the Ikalukrok are recognized in this area: (i) an upper barite interval, (ii) a middle interval of shale with interbeds of calcareous radiolarite, lithic turbidite, and chert, and (iii) a lower sulfide zone.

\section{Fossil Data}

Conodont and radiolarian collections indicate that the age of the Ikalukrok is late Early-Late Mississippian (Osagean to Chesterian) (figs. 4, 5). This age is defined both by samples from the Ikalukrok itself and from the underlying Kivalina unit and Kayak Shale and the overlying Siksikpuk Formation (discussed below). Table 1 lists 39 conodont samples of the Ikalukrok collected from 10 outcrop localities and 27 drill holes. Outcrop samples produced significantly more age-diagnostic faunas than did drill hole collections ( $>60 \%$ versus $<25 \%$ ). Additional age constraints for this unit are provided by 5 radiolarian samples from 2 outcrops and 3 drill holes (table 14, locs. 41, 65, 131, 136, and 151). Conodont biofacies denote platform margin, slope, and (or) basinal settings for the Ikalukrok unit. Sponge spicules and (or) radiolarians replaced by pyrite and (or) phosphate occur in a number of the heavy-mineral concentrates derived from this unit; possible barite, fluorite, and sphalerite were also observed in a few concentrates. 
Red Dog Fossils OF 2/24/06 4:55 PM p. 9

Conodont faunas of late Early Mississippian (Osagean) age were obtained from carbonate layers and concretions in the Ikalukrok unit at several localities. A limestone lens (loc. 4) yielded early to middle Osagean conodonts. Additional samples of lithic turbidite and calcareous radiolarite from outcrop and Su-Lik and Paalaaq drill holes (locs. $47,123,139)$ produced conodonts with a more restricted age of middle Osagean (Sc. anchoralis-D. latus Zone), and a fourth collection (loc. 48) has an age confined to the upper half of that zone. All five of these assemblages denote postmortem transport from or within outer platform, slope, and (or) basinal depositional settings. A lithic turbidite from Anarraaq (loc. 128, sample 3) contains conodonts no older than late Osagean, as well as redeposited older forms (middle Osagean and Kinderhookian).

The Ikalukrok unit has also yielded conodonts and radiolarians of Late Mississippian age. Limestone turbidites(?) in outcrop produced conodonts of probable late Meramecian-earliest Chesterian age (loc. 76). Conodonts from the upper Ikalukrok at Su-Lik (loc. 124) are early Chesterian. Both faunas represent the gnathodid biofacies and imply a slope depositional setting. Combined constraints from conodonts and radiolarians in interbedded calcareous and siliceous radiolarite $\leq 15 \mathrm{~m}$ below the top of the Ikalukrok unit (table 14, loc. 41) imply that these beds are also early Chesterian. The age of the uppermost Ikalukrok unit in drill core is limited by two samples from DDH 50 (table 14, loc. 136). In this section, radiolarians $4 \mathrm{ft}$ below the contact with the overlying Siksikpuk Formation are no older than Chesterian and radiolarians just above the contact are no younger than Morrowan.

Limestone turbidites in a section that may be part of the Ikalukruk unit (loc. 92) yielded a diverse conodont fauna of middle Late Mississippian (early late Meramecian) 
age with several interesting aspects. This section has some lithologic features characteristic of the Kuna Formation in the EMA, but others more typical of the Lisburne Group in the PCA, and its structural level is ambiguous. The fauna, which contains redeposited elements of Osagean age, is likewise transitional between that of the Ikalukrok unit and the PCA Lisburne. It includes the largest single collection of the relatively rare conodont Embsaygnathus asymmetricus Metcalfe, which has been reported only from central Texas, northern and southwestern England, eastern Ireland, west Malaysia, and northern Alaska. Other occurrences of Embsaygnathus and (or) the allied form species Geniculatus claviger (Roundy) in the Brooks Range include a sample from the Kogruk Formation in the EMA (table 6, loc. 160, sample 1) and several collections from the Howard Pass quadrangle to the east (fig. 1). One of these samples is from shallow-water strata of the Lisburne Group in the EMA (table 1, loc. 71 [sample 92AD33-0.2] of Dover and others, 2004); the others are all from deep-water facies of the Lisburne in the PCA and IRA (table 1, loc. 16 of Dumoulin and others, 1993; table 1, locs. 60, 89 [sample 92AD214-44], 91, and 92 of Dover and others, 2004).

Embsaygnathus is a shallow-water, likely warm-water conodont that may be redeposited into deeper water settings; its rarity likely reflects its narrow stratigraphic range in the middle Mississippian

\section{KIVALINA UNIT (TABLE 2; PLATE 1)}

\section{Lithofacies}

The Kivalina unit of the Kuna Formation consists of rhythmically interbedded, black to gray, variably calcareous shale and gray limestone with minor intervals of 
volcanic rocks near the base of the unit. It crops out only in a 19 by $90 \mathrm{~km}$ area around the Red Dog Mine, is a defining feature of the Red Dog plate, and has a maximum thickness in outcrop of at least $122 \mathrm{~m}$ (Moore et al., 1986; Young, 2004). Continuous intervals of the Kivalina unit that are $>500 \mathrm{~m}$ thick have been drilled in the structural hanging wall at Paalaaq (e.g., DDH 777, map 3, loc. 137), but these sections are folded, faulted, and appear to be structurally thickened. Carbonate layers in the Kivalina unit are mainly bioclastic supportstone (grainstone and packstone), calcareous spiculite, and calcareous siltstone.

Depositional patterns, lithofacies, and fossils suggest that the Kivalina unit grades downward and laterally into the Kayak Shale and upward, and perhaps laterally, into the Ikalukrok unit. The Kivalina also grades laterally and (or) upward into unnamed units of the Lisburne Group that are more carbonate-rich than the Kivalina sensu stricto and (or) may have formed in somewhat shallower water. Near the southern limit of the Red Dog plate (loc. 78, map 1), for example, strata lithologically and faunally transitional between typical Kivalina and shallow-water facies of the Utukok Formation crop out.

\section{Fossil Data}

The Kivalina unit in both outcrop and core has yielded few age-diagnostic conodont collections (figs. 4, 5; table 2). More than half of the 28 samples from 5 outcrops and 14 drill holes (table 2) were barren, and many productive samples contain only meager long-ranging faunas. The most precise ages for the Kivalina unit come from three drill holes at and north of Paalaaq (locs. 137, 138, and 142) that yielded conodonts of middle Osagean Sc. anchoralis-D. latus Zone age. All three samples come from 
structurally complex (fault-bounded) sections, so their stratigraphic level within the Kivalina unit is uncertain. The assemblages are all postmortem winnows, and the collection from locality 142 indicates transport into a slope or deeper water depositional setting. Several faunas with broader age ranges also suggest outer shelf, slope, or basin environments (locs. 17, 122, 143).

Bioturbated bioclastic packstone at locality 78 that is transitional between typical Kivalina unit and shallow-water facies of the Utukok Formation produced conodonts of late Osagean-early Chesterian age. The conodonts are a postmortem winnow from a shelf environment.

Phosphatic brachiopod fragments or pyritized bioclasts occur in one quarter of the Kivalina unit heavy-mineral concentrates. A few concentrates contained possible fluorite and (or) sulfide minerals.

The sparse conodont faunas that typify the Kivalina unit may, at least in part, reflect the abundant influx of noncarbonate detritus into the Kuna basin and its environs during Early Mississippian time. Influx of siliciclastic material generally inhibits growth and development of normal marine faunas in carbonate platform settings (e.g., Wilson, 1975). Correlative shallow-marine strata (Utukok Formation and related rocks, tables 7 , 8) are also moderately to very poorly productive of conodonts.

\section{KUNA FORMATION (KEY CREEK PLATE) (TABLE 3; PLATE 1)}

The Kuna Formation is widely exposed in the Key Creek plate of the EMA. It crops out discontinuously from the central De Long Mountains quadrangle south into the Baird Mountains quadrangle and as far east as the western part of the Killik River 
quadrangle (fig. 1). Throughout this extent, it is relatively uniform in character and thickness. In the Red Dog area, it is about 60 to 110 m thick (Young, 2004). No strata similar to the Kivalina unit are seen, and the entire formation resembles the Ikalukrok unit of the Kuna in the Red Dog plate. A gradational depositional contact between the Kuna Formation and the underlying Kayak Shale is widely preserved in the Key Creek plate, both in outcrop (map 1, loc. 103) and subsurface (e.g., DDH 587, northeast of the Main deposit, map 3, loc. 158). The upper contact of the Kuna with the Siksikpuk Formation is also conformable and marked, as in the Red Dog plate, by a color change and, commonly, a horizon of mammiform structures (Young, 2004).

\section{Lithofacies}

The Kuna Formation in the Key Creek plate consists chiefly of siliceous carbonaceous shale and mudstone with subordinate intervals of carbonate and rare volcanic rocks. Mudrocks grade locally to vitreous cherts and are petrographically and chemically similar to mudrocks of the Ikalukrok unit (Slack and others, 2004). Much chert likely originated as radiolarite or siliceous spiculite, but some may be silicified carbonate. Carbonate layers are mostly organic-rich dolostone and lesser calcareous radiolarite and spiculite. Some dolostone layers could be detrital concentrations of dolomite crystals derived from dolomitized platform margin sediments, but most layers probably formed during diagenesis.

\section{Fossil Data}


Conodont and radiolarian collections define an age range of Osagean into the Chesterian (i.e., late Early to late Late Mississippian) for the Kuna Formation of the Key Creek plate in the Red Dog area (figs. 4, 5; table 3; table 14, locs. 72, 100, 107, 112). Calcareous spiculite and organic-rich dolostone from the lower and middle parts of the Kuna at two localities (locs. 24, 103) yielded conodonts of middle Osagean (Sc. anchoralis-D. latus Zone) age. Assemblages from locality 103 (2 samples) suggest a slope depositional environment and include a few redeposited shallow-water forms (Eotaphrus burlingtonensis). The dolostone sample, taken about $115 \mathrm{ft}$ below the top of the Kuna Formation (loc. 24), produced a collection that likely denotes a basinal setting. Overlapping ranges of conodonts and radiolarians in interbeds of calcareous and siliceous radiolarite $\sim 50 \mathrm{ft}$ below the top of the Kuna Formation (loc. 107 in tables 3 and 14) indicate that these beds are early Chesterian. At this same locality, greenish-gray chert of the overlying Siksikpuk Formation was sampled $\sim 150 \mathrm{ft}$ above the contact with the Kuna Formation, which is marked here both by mammiform structures and a color shift, and contained radiolarians of probable Morrowan (Early-early Middle Pennsylvanian) age (discussed further below under "Etivluk Group").

\section{DEEP-WATER FACIES OF THE LISBURNE GROUP IN THE PICNIC CREEK}

\section{ALLOCHTON (TABLE 4, PLATES 1, 2)}

Strata of the Picnic Creek allochthon in the Red Dog area occur in two plates, the Wulik and Amaruk (Young, 2004), which are generally equivalent to the sequences of these names of Mayfield and others (1988) (fig. 3). The Lisburne Group in both plates is in part similar to the Kuna Formation and consists mainly of chert, carbonate, and shale, 
but no formal formation names have been applied to most of these rocks. In both plates, the Lisburne gradationally overlies (and may in part laterally grade into) the Kayak Shale and conformably or disconformably underlies the Siksikpuk Formation (fig 4; Curtis and others, 1984; Ellersieck and others, 1990).

\section{Lithofacies}

The Lisburne Group of the Wulik plate is well exposed northeast of Sheep Mountain in the southeastern De Long Mountains quadrangle and in the western Misheguk Mountain quadrangle (map 1, locs. $34,35,117$ ). The Lisburne in this plate is at least $140 \mathrm{~m}$ thick and resembles the Kuna Formation but contains more carbonate than is typical of most Kuna sections. We interpret the carbonate beds as turbidites derived from a carbonate platform margin.

According to Mayfield and others (1988) and Ellersieck and others (1990), the Lisburne Group of the Amaruk plate consists of $80 \mathrm{~m}$ or less of limestone, sandy limestone, and local sandstone (which they call Utukok and Kogruk Formations) overlain by as much as $200 \mathrm{~m}$ of chert and carbonate. At locality 7 (map 1), the upper part of this sequence is spiculitic siliceous mudstone and chert with carbonate supportstone interbeds. Field relations and sedimentary structures suggest that the carbonates formed as turbidites and (or) grain-flow deposits.

Additional strata in the southern De Long Mountains quadrangle are included in the Picnic Creek allochthon based on recent mapping (De Vera and others, 2004; Young, 2004), although they were previously considered part of the Ipnavik River allochthon (Mayfield and others, 1990). These rocks are probably part of the Amaruk plate. At 
Red Dog Fossils OF 2/24/06 4:55 PM p. 16

localities 19 and 20 (map 1), the Lisburne Group is 150 to $200 \mathrm{~m}$ thick and consists of interbedded chert and dolostone

Fossil Data

Conodont collections indicate that in the Red Dog area, the Lisburne Group of the PCA is roughly coeval with the Kuna Formation in the Endicott Mountains allochthon (figs. 4, 5). Twenty-one samples from 11 outcrop localities are listed in table 4.

Diagnostic assemblages come chiefly from the Wulik plate (locs. $34,35,37,38,117$ ) but also occur in Lisburne strata in the Amaruk plate (locs. 10, 19, 20). Conodonts from a measured section (loc. 34) indicate that the basal $360 \mathrm{ft}(\sim 120 \mathrm{~m})$ of the Lisburne in the Wulik plate is Early Mississippian (late Kinderhookian-Osagean). More precise ages of middle Osagean (Sc. anchoralis-D. latus Zone) come from lithologically similar strata to the south and east in this plate (locs. 37, 38, 117). A sample near the top of the Lisburne at loc. 34 contains conodonts of middle Late Mississippian (late Meramecian) age. A fauna from the highest carbonate beds of the Lisburne in this area (loc. 35) is no older than early Chesterian and includes reworked conodonts of early and middle Osagean age. Conodonts indicate that at least part of the Lisburne section in the Amaruk plate is no younger than Osagean (loc. 10) whereas other parts of the section in this plate are late Meramecian (locs. 19, 20).

Conodont assemblages from the Lisburne Group in both the Wulik and Amaruk plate generally represent postmortem winnows into quiet, deep-water environments. Some samples (e.g., loc. 34, sample KE98-24 (+382 ft)) contain conodonts derived from a shallow-water setting, but they occur with rare radiolarians and common sponge 
spicules that indicate a deep-water depositional environment. Phosphatic and phosphatized rock fragments and bioclasts, including locally notable sponge spicules and (or) radiolarians, occur in many of the heavy-mineral concentrates from this unit. Several concentrates contained minor to abundant fluorite.

\section{OTHER DEEP-WATER STRATA OF THE LISBURNE GROUP (TABLES 5, 14;}

\section{PLATE 2)}

In addition to strata of the PCA just described, deep-water facies of the Lisburne Group that resemble the Kuna Formation also occur in the Wolverine Creek plate of the EMA and throughout the IRA. These facies are a subordinate component of the Lisburne in the Wolverine Creek plate, where they are intercalated between shallow-water strata of the Utukok and Kogruk Formations, but make up the entire Lisburne Group in the IRA (fig. 4).

\section{Lithofacies}

An interval of black shale similar to the Ikalukrok unit of the Kuna Formation locally overlies the Utukok Formation in exposures of the Wolverine Creek plate in the Rok window. This unit is at least $30 \mathrm{~m}$ thick at localities 88 and 89 (map 1) but thins to the north and west. The shale contains thin interbeds of finely crystalline organic-rich dolostone and calcareous and siliceous spiculite with rare radiolarians.

In the Red Dog area, Lisburne Group strata of the IRA occur only in the northwestern Misheguk Mountain quadrangle (De Vera and others, 2004; Nachralik Pass sequence of Curtis and others, 1984) (fig. 3). Formal formation names have not been 
applied to these rocks, which comprise less than $100 \mathrm{~m}$ of chert and lesser dolostone intruded by rare diabase sills and dikes (Curtis and others, 1984). These strata were sampled at locality 120 (map 1), where they are mainly spiculitic chert with rare interbeds of dolomitic calcareous radiolarite. As in other allochthons described above, the Lisburne Group here overlies the Kayak Shale and underlies the Siksikpuk Formation (Fig. 4, Mayfield et al., 1988).

\section{Fossil Data}

Lisburne Group facies in the Wolverine Creek plate that resemble the Kuna Formation are of Osagean to at least Meramecian age (figs. 4, 5; tables 5, 14). Organicrich dolostone contains conodonts that are no younger than Osagean (loc. 85). Calcareous spiculite produced late Meramecian-Chesterian conodonts; the assemblage includes transported shallow-water forms that probably accumulated in a middle platform or deeper water environment (loc. 89). Poorly preserved radiolarians from cherty carbonate and siliceous mudstone in this section are of poorly constrained late Paleozoic age (table 14, loc. 88).

Only a single diagnostic fossil collection is known from the Lisburne Group of the IRA in the Red Dog area (figs. 4, 5). Dolomitic calcareous radiolarite (loc. 120) produced middle Osagean (Sc. anchoralis-D. latus Zone) conodonts that have been transported from or within a platform and/or slope setting.

\section{KOGRUK AND UTUKOK FORMATIONS AND RELATED ROCKS}


In the Red Dog area, shallow-water facies of the Lisburne Group occur in the Wolverine Creek plate and locally in the Key Creek plate of the EMA, and throughout the KRA, and comprise two formations. The lower unit, the Utukok Formation, is mostly impure limestone that weathers a distinctive yellowish orange. Abundant noncarbonate detritus, chiefly mud and quartz silt and sand, occurs throughout the Utukok, both as discrete beds and disseminated within carbonate layers, but the unit also contains intervals of relatively pure limestone. The overlying Kogruk Formation is a clean gray carbonate that is locally extensively altered to dolostone and chert. A thin interval of deep-water strata similar to the Kuna Formation makes up the uppermost part of the Lisburne in much of the EMA and KRA; these rocks are generally (and herein) included in the Kogruk Formation, but have been called Kuna or Tupik Formation in parts of the KRA (Sable and Dutro, 1961; Curtis and others, 1984; Mayfield and others, 1990).

The stratigraphic context of shallow-water Lisburne Group sections differs somewhat between allochthons (fig. 4). Whereas the Lisburne Group in the EMA overlies the Endicott Group, coeval strata in the KRA overlie Devonian carbonate rocks. The Lisburne Group in both allochthons underlies the Siksikpuk Formation.

Lisburne Group strata of the Wolverine Creek plate are exposed chiefly in two windows (Young, 1992): the Mt. Raven window north of Red Dog and the Rok window to the south, west, and northwest. Lisburne Group rocks from this plate also form large olistoliths along the boundary between the Wolverine Creek and Red Dog plates; several of these olistoliths are exposed adjacent to the Red Dog Mine, and others have been penetrated by drill holes in the Main and Aqqaluk deposits. 
Shallow-water facies of the Lisburne Group occur in the Key Creek plate west, south, and east of the Red Dog Mine. At all of the localities we studied, deep-water strata similar to the Kuna Formation overlie and (or) grade laterally into the shallow-water facies.

Five plates are recognized in the KRA at three structural levels; these are, from structurally lowest to highest, the Chimney, Amphitheatre, Wulik Peaks, Kelly, and Eli (figs. 3, 4; Young, 2004). Our reconnaissance studies suggest that the lithofacies, biofacies, and thickness of the Lisburne differ somewhat from plate to plate; these differences are summarized below.

Pyrobitumen is a notable component of the Kogruk Formation, particularly in the Wolverine Creek plate, and it has also been noted locally in the Utukok Formation. It commonly occurs along fractures, in vugs, and between dolomite crystals in Kogruk samples from the Rok and Mt. Raven windows and in Kogruk(?) olistoliths in the Red Dog mine area. In some olistolith samples pyrobitumen fills fossil molds in dolostone and chert; textural evidence suggests two episodes of hydrocarbon migration may have affected these rocks (R. Burruss, written commun., 2002). Pyrobitumen lines vugs and fractures in some Kogruk samples from the Key Creek plate (Port Road succession, fig. 1) and occurs in fractures and vugs in the Kogruk of the Kelly River allochthon at a few localities in the eastern De Long Mountains quadrangle. Fractures filled with pyrobitumin and calcite have also been found in the Utukok Formation of the Wulik Peaks plate (J. Kelley, written commun., 1997). 


\section{Lithofacies}

Wolverine Creek plate, EMA: The Kogruk Formation in the Rok window consists of $\sim 70$ to $100 \mathrm{~m}$ of limestone, dolomitic limestone, and dolostone (map 1, locs. 73, 75, $82,84,87)$. Similar strata occur as olistoliths that were penetrated in more than 50 drill holes in the Main and Aqqaluk deposits (map 3, locs. 147, 148).

In the Mt. Raven window only the upper half of the Lisburne Group (Kogruk Formation) is exposed. The section is greatly thickened by folding and repeated in multiple thrust imbricates. Most of the section contains sedimentary features and bioclasts indicative of a shallow-water setting (map 1, locs. 44, 45, 68). The uppermost Kogruk in this area (map 1, locs. 42, 43?) consists of thinly interbedded dark-gray locally phosphatic carbonate and black spiculitic chert.

Key Creek plate, EMA: In the subsurface Port Road succession (fig. 1; map 4, locs. 159, 160), the Kogruk Formation is 100 to $150 \mathrm{~m}$ thick and is mainly white to lightgray dolostone with a 30-cm thick layer of green mudstone near the base. An interval of cavernous porosity several meters thick was encountered $9 \mathrm{~m}$ below the top of the unit in DDH 1104 (loc. 160). In this drill hole, the Kogruk is overlain by $14 \mathrm{~m}$ of black noncalcareous shale that resembles the Kuna Formation; the nature of the contact is uncertain but could be conformable. Brownish-gray shale of the Siksikpuk Formation is faulted above the top of the black shale.

The Kogruk and Kuna Formations appear to interfinger in the southwestern Misheguk Mountain quadrangle (map 1, loc. 121). The Kogruk here is massive, light- 
gray-weathering, dolomitic limestone with local chert; pelmatozoan supportstone is the predominate lithology.

$K R A$ : The Chimney plate is exposed chiefly as large olistoliths that underlie much of the Wulik Peaks plate along the south and north flanks of the Wulik Peaks (map 1). Mayfield and others (1990) included these strata in their Kelly sequence. The Lisburne Group in this plate has been little studied; most outcrops are limestone, dolostone, and chert of the Kogruk Formation (map 1, locs. 2, 21-23).

Rocks of the Amphitheatre plate (Amphitheatre sequence of Curtis and others, 1990) are exposed northeast of the Red Dog Mine and may be a lateral structural equivalent of the Chimney plate (fig. 3). The Kogruk Formation in this succession (map 1, loc. 99) is more than $600 \mathrm{~m}$ thick (Armstrong, 1970) and consists of limestone that is locally dolomitic and (or) cherty. Sparsely exposed black shale and subordinate phosphatic limestone (Kuna Formation of Curtis and others, 1990) forms the uppermost part of the Lisburne in this plate (map 1, loc. 94).

Strata referred to as Wulik Peaks plate by Young (2004) were included in the Kelly River sequence by Mayfield and others (1990) and crop out throughout the Wulik Peaks (map 1, locs. 6, 32, 40, 46, 56, 60, 62). This plate may be a lateral structural equivalent of the Kelly plate (fig. 3). The Kogruk Formation is $>550$ m thick and is similar to the Kogruk in the Amphitheatre plate but contains slightly less dolomite and more chert overall. The uppermost Kogruk is only locally exposed (map 1, locs. 5, 8) and consists of a thin interval of phosphatic black shale and chert (Kuna Formation of Mayfield and others, 1990). 
In the Red Dog area, the Kelly plate is exposed in several thrust sheets northeast of the Red Dog Mine. These sheets are south of, and locally thrust above the Amphitheater plate. The Kogruk Formation (200 to 600 m thick) resembles the Kogruk in other plates of the Kelly River allochthon (map 1, loc. 69, 110). The top of the Kogruk is generally a fault or a recent erosional surface, but where the contact with the overlying Etivluk Group is exposed, the uppermost Kogruk consists of black shale interbedded with phosphatic limestone (map 1, loc. 116).

Strata of the Eli plate (Eli sequence of Mayfield et al., 1988) crop out southwest, east, and southeast of the Red Dog Mine, in the Noatak, Misheguk Mountain, and Baird Mountains quadrangles (Fig. 1). The Lisburne Group in this plate has been studied in detail only in the Baird Mountains quadrangle (Dumoulin and Harris, 1992), where it is most similar to the Lisburne of the Kelly plate.

\section{Fossil Data}

Conodonts indicate an age of Osagean-Chesterian for the Kogruk Formation in the Red Dog area (figs. 4, 6). Forty-eight conodont samples were taken from 41 localities in the Kogruk (table 6; table 13, locs. 161, 162). In addition, carbonate breccia in the Okpikruak Formation produced a conodont assemblage of mixed age derived in part from a Mississippian source (table 9, loc. 62), most likely the Kogruk Formation or related rocks. Kogruk collections were made from outcrop and drill holes in both the Key Creek and Wolverine Creek plates of the EMA and from outcrops of the Chimney, Amphitheatre, Wulik Peaks, and Kelly plates of the KRA. Only 3 of these samples were barren, and more than half produced biostratigraphically useful faunas. Phosphatic and 
phosphatized rock fragments and bioclasts (including phosphatic brachiopods and ichthyoliths, as well as phosphatized bryozoan and crinoid fragments and spine, ostracode, gastropod, and pelecypod steinkerns) are a notable component of many of the heavy-mineral concentrates from this unit, particularly those from the uppermost part of the Kogruk. Minor glauconite also occurs in two concentrates from the uppermost Kogruk in the KRA.

Wolverine Creek plate, EMA: Table 6 includes 13 samples from 12 localities in the Kogruk of the Wolverine Creek plate; conodonts indicate that the unit is largely Meramecian-early Chesterian but is, in part, locally Osagean (figs. 4, 6). Olistoliths of brecciated dolostone that resemble the Kogruk Formation were sampled in the Aqqaluk (DDH 550, loc. 147) and Main (DDH 605, loc. 148) deposits and produced conodonts of Kinderhookian-Osagean age that are typical of a high-energy, shallow-water environment. Dolomitic skeletal packstone at the base of the Kogruk Formation in the northwestern part of the Rok window yielded late middle-early late Osagean conodonts characteristic of a platform depositional setting (loc. 73 [KE98-25B]). Crinoidal dolostone in the upper part of the Kogruk in both the Mt. Raven and Rok windows contains conodonts that denote shallow-water, partly restricted to high-energy environments (locs. 45, 75). Dolostone with phosphatized bioclasts at the top of the unit in both windows (locs. 42, 73[KE98-25A]) yielded conodont faunas that indicate slope and outer platform or deeper settings, respectively. The age of the uppermost Kogruk is best constrained in the Rok window, where both shallow-water and overlying deeper water conodont faunas are likely of very early Chesterian age (figs. 4, 6). Equivalent 
collections from the Mt. Raven window produced less precise ages of late Meramecianearly Chesterian. A single sample from the Rok window, from strata thought to be upper Kogruk, yielded atypically old conodonts of late Late Devonian-early Early Mississippian age that may have been reworked from underlying strata (loc. 82).

Key Creek plate, EMA: Four samples from 3 localities in table 6 (locs. 121, 159, 160) constrain the age of the Kogruk Formation in the Key Creek plate. Conodonts from the upper part of the Kogruk in the Port Road succession (loc. 160) are late Osageanearly Meramecian, possibly early Meramecian; taphonomy of this assemblage implies a relatively shallow-water setting. In the southwestern Misheguk Mountain quadrangle (loc. 121), dolomitic pelmatozoan supportstone (Kogruk Formation?) that appears to grade laterally into the Kuna Formation yielded a Meramecian-early Chesterian conodont assemblage that suggests an inner to middle platform depositional setting.

KRA: Tables 6 and 13 include 31 conodont samples from 26 localities in the Kogruk Formation of the KRA. Collections represent the Chimney (5), Amphitheatre (3), Wulik Peaks (10), and Kelly (4) plates; 7 samples were taken from probable KRA strata for which the plate is uncertain. Fossil data presented here and elsewhere denote an age of late Osagean to Meramecian for most of the Kogruk in the KRA (Dumoulin and others, 2004), but the upper part of the unit is at least locally Chesterian (figs. 4, 6). Age-diagnostic conodont faunas from the Kogruk Formation in the KRA are chiefly Late Mississippian. Conodonts from the basal(?) Kogruk of the Chimney plate are no younger than Osagean (loc. 21), but higher beds in this plate are of probable late 
Meramecian age (loc. 22). Late Meramecian conodonts occur in samples from the Wulik Peaks plate (locs. 6, 40) and faunas of late Meramecian-Chesterian age were obtained from the Wulik Peaks (locs. 32, 46, 62) and Kelly (loc. 69; table 13, loc. 162) plates. KRA strata for which the structural plate is uncertain also produced conodonts of late Meramecian-Chesterian (locs. 113, 114) age. Conodont assemblages from the Kogruk in the Chimney, Wulik Peaks, and Kelly plates suggest deposition in shallow to moderate water depths with locally restricted circulation and, in the Chimney plate, some highenergy conditions.

The uppermost Kogruk Formation in the Kelly River allochthon is at least in part of late Late Mississippian (Chesterian) age. Dark limestone, chert, and (or) shale that locally occur at the top of the unit contain early Chesterian conodonts in the Amphitheater and Kelly plates (locs. 94, 116). Conodonts of late Meramecian-very early Chesterian age that indicate the gnathodid biofacies are found at the top of the Kogruk in the Wulik Peaks plate (loc. 8). All of these strata, like equivalent rocks in the upper Kogruk of the EMA discussed above, formed in a relatively deep-water setting (Dumoulin and others, 2004). The facies shift recorded in these beds is part of a regional drowning of carbonate platforms that occurred throughout the western and west-central Brooks Range in latest Meramecian to earliest Chesterian time (Dumoulin and Harris, 1992; Dumoulin and others, 2004).

A single collection from bioclastic supportstone confidently assigned to the Kogruk Formation but of uncertain structural position (loc. 93) produced conodonts of late Chesterian (Rh. muricatus Zone through Rh. primus Zone) age that suggest derivation from a relatively shallow-water setting. These rocks are on trend with and 
adjacent to strata of the KRA, but occur close to a major fault zone (Curtis and others, 1990). The CAI of this assemblage (3.5-4) is considerably higher than that from collections on trend to the southwest and northeast (e.g. CAI=2-2.5 and $\leq 2.5$ at locs. 94 and 116). Whatever the structural level of these late Chesterian strata, they indicate that shallow-water platform carbonate deposition recurred in, or perhaps persisted into, latest Mississippian time in at least some parts of the De Long Mountains area.

\section{UTUKOK FORMATION AND RELATED ROCKS (TABLES 7, 8, 13; PLATES 2, 3)}

\section{Lithofacies}

Wolverine Creek plate, EMA: In the Rok window, the Utukok Formation consists of $\sim 120 \mathrm{~m}$ of limestone, locally argillaceous and (or) sandy, interbedded with shale, calcareous shale, and siltstone (map 1, locs. $63,64,73,74,83$ ). Limestone, in wavy to nodular partly bioturbated beds 5 to $20 \mathrm{~cm}$ thick, includes skeletal wackestone, packstone, and grainstone. Some samples contain $>10-15$ percent non-calcareous mud, silt, and sand; coarser detritus is mainly quartz and lesser white mica. Throughout the Utukok in this plate, zones rich in lime mud are partly altered to chert and (or) dolomite, and the uppermost beds of the formation are often pervasively dolomitized and (or) silicified.

Key Creek plate, EMA: In the Port Road succession, the Utukok Formation is about $150 \mathrm{~m}$ thick and conformably overlies the Kayak Shale (DDH 1103, map 4, loc. 159). It comprises light- to medium-gray limestone that is locally partly silicified and (or) partly dolomitized and has 5-15 percent gray to black shale interbeds that are $2 \mathrm{~mm}$ - 
$2.5 \mathrm{~m}$ thick. Skeletal packstone and grainstone makes up much of the unit, but sparsely bioclastic wackestone is common in shale-rich intervals. Shale layers contain trace amounts of quartz silt and sand.

The Utukok Formation also occurs in the Key Creek plate west of the Red Dog Mine (map 1, loc. 31). Here, it consists of orange-weathering limestone with notable interbeds of shale and (or) sandstone and overlies the Kayak Shale. Similar rocks in the Key Creek plate south of the Red Dog Mine (map 5, loc. 172) were provisionally included in the Utukok by Dumoulin and others (2004).

$K R A$ : No strata equivalent to the Utukok Formation have been recognized in the Chimney plate. The lower part of the Lisburne Group in the Amphitheatre plate is a distinctive sequence of dark-gray, argillaceous limestone and carbonaceous shale (micritic limestone unit of Curtis et al., 1990). These rocks (map 1, locs. 95, 96, 98, and 99) are coeval with and resemble the Utukok in some ways but have not been formally included in that unit. The Utukok in the Wulik Peaks plate is mostly limestone with locally abundant shale and sandstone interbeds (map 1, locs. 40, 52-56, 62, 81). Shale is less carbonaceous than that in the Utukok of the Amphitheater plate. Sandstone generally contains $\leq 30-40$ percent non-carbonate detritus, but siliciclastic-dominated sandstones occur locally in the southern Wulik Peaks (Mayfield et al., 1990). The Lisburne Group in the Kelly plate (map 1, locs. 9, 70,102) generally resembles that in the Wulik Peaks plate. However, the Utukok in the Kelly plate has less interbedded shale, more noncarbonate silt and sand, and more diverse bioclasts than coeval Wulik Peaks sections. The Utukok in the Kelly plate may reach $1000 \mathrm{~m}$ in thickness, according to Curtis and 
others (1990). The Utukok in the Eli plate is at least $180 \mathrm{~m}$ thick and consists of limestone, quartzose to calcareous sandstone, and subordinate siltstone and shale. In the Baird Mountains, siliciclastic rocks make up about 40 percent of the section and include an interval of relatively pure quartz arenite that is $10 \mathrm{~m}$ thick and at least $12 \mathrm{~km}$ long.

\section{Fossil Data}

Conodonts and other fossils suggest that the Utukok Formation in the Red Dog area is chiefly Kinderhookian and Osagean (figs. 4, 6; Dumoulin and others, 2004). Thirty-three conodont samples from 28 localities in the Utukok are included in this study (table 7; table 13, loc. 166). These collections come from the Wolverine Creek and Key Creek plates of the EMA, the Wulik Peaks, Kelly, and Eli plates of the KRA, and six localities for which the structural level is unknown. An additional 6 samples from 5 localities (table 8) were taken from rocks that may correlate with the Utukok Formation in the Key Creek plate (EMA) and Amphitheatre plate (KRA). About one-quarter of our samples from the Utukok and related strata were barren, and less than half produced relatively tightly constrained ages. Almost two-thirds of the heavy-mineral concentrates from the Utukok samples contained phosphatic, phosphatized, and (or) pyritized bioclasts including ichthyoliths and phosphatic brachiopods, and phosphatized foraminifers, ostracodes, spines, and bryozoan and pelmatozoan fragments. A few concentrates contained fluorite.

Wolverine Creek plate, EMA: Table 7 includes 6 samples from the Utukok Formation in the northwestern part of the Rok window. The most diagnostic collections 
are of Osagean age and indicate a shallow-water, locally restricted depositional setting (locs. 63, 64, 73).

Key Creek plate, EMA: Five samples (tables 7, 8) constrain the age of the Utukok Formation and possibly stratigraphically equivalent rocks in the Key Creek plate. A collection $\sim 30$ m below the top of the Utukok in the subsurface Port Road succession (DDH 1103, sample 3, loc. 159) is middle to middle late Osagean and represents postmortem transport within or from a high-energy depositional setting. Conodonts from skeletal supportstone near the base of the Utukok in outcrop west of the Red Dog Mine (loc. 31) are also middle to middle late Osagean. Pelmatozoan-bryozoan grainstone from a similar stratigraphic level in the Noatak quadrangle (table 8 , loc. 172) produced Osagean, possibly middle to late Osagean, conodonts.

KRA: Conodont collections from the Utukok Formation in the KRA reported here include 10 samples from the Wulik Peaks plate, 3 samples from the Kelly plate, and 2 samples from the Eli plate. The oldest faunas, of middle to late Kinderhookian age, come from the Eli plate (loc. 167; table 13, loc. 166). Several olistoliths of uncertain structural provenance (locs. 18, 25) yielded coeval conodonts. One of the Eli plate collections (loc. 167) indicates a high-energy, shallow-water depositional regime, whereas the olistolith faunas suggest postmortem transport into middle shelf or deeper, relatively quiet water. Most Utukok Formation conodont assemblages from the KRA are of Osagean age and imply shallow-water, locally restricted to high-energy environments. A fauna from the Kelly plate indicates an early Osagean age (loc. 102). Other collections, from the Kelly (loc. 9) and Wulik Peaks (loc. 52) plates, are middle-late Osagean and one Wulik Peaks sample (loc. 62) is no older than late Osagean. Foraminifers imply that the uppermost 
Red Dog Fossils OF 2/24/06 4:55 PM p. 31

Utukok in parts of the Wulik Peaks plate could be as young as early Meramecian (Dumoulin and others, 2004) and a lithostrotionid coral from correlative beds of uncertain structural position (loc. 57) is also Late Mississippian. Strata equivalent to the Utukok in the Amphitheater plate (table 8, locs. 95, 96, 98, and 99) have produced no precisely dated conodonts or other fossils (Dumoulin and others, 2004).

\section{ETIVLUK GROUP AND OKPIKRUAK FORMATION}

\section{ETIVLUK GROUP (TABLES 9, 14)}

The Pennsylvanian-Jurassic Etivluk Group (Mull and others, 1982) overlies the Lisburne Group in all allochthons that contain sedimentary rocks (figs. 2, 4) and includes the Siksikpuk and overlying Otuk Formations (fig. 2). These formations are relatively easy to differentiate in the EMA, but can be difficult to distinguish in other allochthons. Mull and others (1987) defined the Imnaitchiak Chert to encompass chert-dominated sections correlative with both the Siksikpuk and the Otuk Formations in these higher allochthons.

\section{Siksikpuk Formation}

In the western Brooks Range, the Siksikpuk Formation in the EMA (Red Dog and Key Creek plates) is $\sim 70$ to $100 \mathrm{~m}$ thick and consists of four informal subunits (Young, 2004). The lower subunit (20 to $40 \mathrm{~m}$ thick) is bright-orange-weathering, dark-gray to blue-gray chert that grades upward to black shale and chert overlain by light-gray siliceous shale and chert. Local layers of brown-weathering carbonate rock-mostly coarse-crystalline iron-rich dolostone-also occur in this subunit. Laminated to thin- 
bedded greenish-gray and maroon aluminous shale and lesser chert (23 to $46 \mathrm{~m}$ thick) makes up the middle subunit. The upper subunit ( 9 to $46 \mathrm{~m}$ thick) comprises greenishgray and maroon chert. A transitional subunit $(\leq 8 \mathrm{~m})$ at the top of the Siksikpuk consists of laminated green-gray, brown-gray, and lesser maroon shale and subordinate chert with a few thin lenses of chert-rich siltstone; these rocks conformably underlie and locally are interbedded with the basal Otuk Formation. Minor barite, as layers, rosettes, and veins, occurs throughout the Siksikpuk. The Siksikpuk in the Wolverine Creek plate of the EMA resembles correlative strata in the Red Dog and Key Creek plates but is thicker (up to $165 \mathrm{~m}$ ) and may contain siltstone in the lower subunit (Young, 2004).

The informal subunits of the Siksikpuk Formation described by Young (2004) can also be recognized in at least some parts of the Kelly River allochthon. In other allochthons, however, the Siksikpuk generally consists of 10 to $30 \mathrm{~m}$ of chert that cannot be readily subdivided and is difficult to distinguish from the Otuk Formation.

Mainly Pennsylvanian and Permian ages have been reported from the Siksikpuk Formation and equivalent strata of the lower Etivluk Group (or lower Imnaitchiak Chert) in the western Brooks Range (Curtis and others, 1984, 1990; Ellersieck and others, 1984, 1990; Mayfield and others, 1984; Dover and others, 2004; Young, 2004). Our data constrain the age of the lower subunit of the Siksikpuk in the EMA (Key Creek and Red Dog plates) and the middle(?) subunit of the Siksikpuk in the KRA (Wulik Peaks plate) (fig. 7). Radiolarians (table 14, locs. 72, 107 [97AK74O-S]) indicate an age of late Late Mississippian to early Middle Pennsylvanian (Chesterian to Morrowan) for samples of predominantly gray chert from the base to $\sim 10 \mathrm{~m}$ above the base of the lower subunit in the Key Creek plate. Grayish-green chert $46 \mathrm{~m}$ above the base of the Siksikpuk, from 
Red Dog Fossils OF 2/24/06 4:55 PM p. 33

strata lithologically transitional between the lower and middle subunits (table 14, loc. 107 [97AK74T]), contains radiolarians of probable Early-early Middle Pennsylvanian (Morrowan) age. Roughly coeval but less diagnostic radiolarian faunas indicate a slightly broader age range of Late Mississippian-early Middle Pennsylvanian (Meramecian to Morrowan) for drill core samples from equivalent strata (gray to black chert) in the Red Dog plate (table 14, locs. 136, 150). Brownish-gray carbonate in the basal Siksikpuk Formation in the Wolverine Creek plate of the EMA (table 9, loc. 67) was sampled for conodonts but was barren; this carbonate layer resembled altered (calcitized) volcanic rock in thin section. A section of maroon and light-greenish-gray shale and lesser chert that may represent the middle subunit of the Siksikpuk in the KRA (Wulik Peaks plate) (table 14, loc. 61) produced late Early-middle Middle Permian (middle Leonardian-middle Guadalupian) radiolarians.

\section{Otuk Formation}

The Otuk Formation in the EMA in the western Brooks Range is $\sim 45 \mathrm{~m}$ thick and, like the Siksikpuk Formation, can be divided into four informal subunits (Young, 2004) that generally correlate with the shale, chert, limestone, and Blankenship members of Mull and others (1982) and Blome and others (1988). The lower subunit (generally 9 to $26 \mathrm{~m}$ thick) consists of color-banded black and greenish-gray shale, lesser chert, and minor rusty-weathering dolostone that contains beds, rosettes, and needles of barite. Gray chert makes up most of the middle subunit, ( $\sim 20 \mathrm{~m}$ thick) with a distinctive recessive zone ( 1 to $5 \mathrm{~m}$ thick) of laminated black chert and shale in the middle of the subunit. The upper subunit is generally thin or absent in the Red Dog plate, but may 
reach a thickness of $15 \mathrm{~m}$ in the Wolverine Creek plate (Young, 2004). It is mainly yellow- to buff-weathering dolostone, limestone, and limy chert with locally abundant monotid pelecypods. The Blankenship member, the uppermost subunit of the Otuk Formation, occurs locally in the Red Dog plate and comprises 3 to $6 \mathrm{~m}$ of laminated black chert and shale.

In higher allochthons, the Otuk Formation generally consists mainly of chert and the subunits recognized in the EMA can be difficult to discern. In the Kelly River allochthon, however, the recessive zone of the middle subunit is present at least locally and the subunits described above can be distinguished.

Fossils of Triassic and (locally) Jurassic age are known from the Otuk Formation and correlative strata assigned to the upper Etivluk Group or upper Imnaitchiak Chert (Curtis and others, 1984, 1990; Ellersieck and others, 1984, 1990; Mayfield and others, 1984, 1990; Blome and others, 1988; Dover and others, 2004). Young (2004) reported inferred age ranges of Early-Middle Triassic (early Smithian to Ladinian) for the lower subunit, Middle(?)-Late Triassic (Ladinian? to Norian) for the middle subunit, middle Late Triassic (middle to late Norian) for the upper subunit, and Early-early Middle Jurassic (Sinemurian to middle Bajocian) for the Blankenship member. The uppermost part of the Imnaitchiak Chert yields radiolarians of Early Jurassic (Hettangian or Sinemurian and Pliensbachian or Toaracian) age in the Howard Pass quadrangle (Dover and others, 2004).

Data presented in this report limit the age of the Otuk Formation in the EMA, PCA, and KRA, and agree with the age distributions outlined above (fig. 7). In the Red Dog plate of the EMA, dark-gray chert interbedded with shale in the middle(?) subunit of 
the Otuk Formation produced radiolarians of late Carnian to middle Norian age (table 14, loc,. 152). Interbedded chert and dolostone in the upper subunit in this plate yielded Monotis(?) sp., conodonts, and radiolarians; the radiolarians restrict the age of these strata to middle Late Triassic (late middle-early late Norian) (tables 9, 14, loc. 66). Radiolarians of late Middle-early Late Triassic (late Ladinian to early Carnian) age were obtained from a drill core sample of gray chert from the middle subunit of the Otuk (below the recessive marker) in the Wolverine Creek plate (table 14, loc. 126). Conodont samples from cherty carbonate in the upper subunit of the Otuk at this locality and a nearby drill hole (table 9, locs. 126, 127) were barren.

Etivluk Group sections from the PCA sampled for this report consist mainly of chert and produced faunas correlative with those from the middle and upper subunits of the Otuk Formation in the EMA. Samples of gray-green chert from the Wulik Peak plate of the PCA yielded radiolarians of late Middle Triassic (probable middle to late Ladinian) and early Late Triassic (probable early to middle Carnian) ages (table 14, loc. 35). Late Late Triassic (late Norian or Rhaetian) radiolarians were found in grayish-black siliceous mudstone in this plate (table 14, loc. 33). Buff-colored bivalve wackestone that contains Late Triassic bivalves (monotids and Palaeocardita(?) sp.) as well as conodonts of probable middle Late Triassic age (table 9, loc. 36) is also most likely part of the PCA; the conodonts in this collection indicate a slope or basin depositional setting.

A section of Otuk Formation in the Wulik Peaks plate of the KRA was also sampled for this study (table 14, loc. 5). Gray chert from the middle subunit, below the recessive marker, produced Middle Triassic (middle to late Ladinian) radiolarians; gray 
and white chert from the middle(?) subunit, above the recessive marker, contained early Late Triassic (early to middle Carnian) radiolarians.

Heterolithic carbonate breccia (table 9, loc. 62) from the Cretaceous-Jurassic Okpikruak Formation (further discussed below) yielded a mixed-age conodont collection derived in part from a Triassic source that is most likely the lower subunit of the Otuk Formation. The conodonts are of middle Early Triassic (Smithian) age and normalmarine, middle-shelf or deeper water biofacies. Slightly younger (latest Early Triassic; latest Spathian) conodonts are the oldest fossils known from the Otuk in the De Long Mountains area (Mayfield and others, 1990); this fauna comes from the EMA (Red Dog plate) (Young, 2004). However, Bodnar (1984) reported conodonts of latest Dienerianearliest Smithian, middle Smithian, and late Smithian age from the lower (shale) subunit of the Otuk (EMA) in the north-central Brooks Range. The outcrop of the Okpikruak that yielded the carbonate breccia appears to be part of the KRA, but the structural unit that produced the Triassic conodonts is unknown.

\section{OKPIKRUAK FORMATION (TABLE 9)}

The Okpikruak Formation, of Jurassic-Cretaceous age, is a sequence of mudstone, siltstone, sandstone, and local polymict conglomerate at least $300 \mathrm{~m}$ thick that was deposited during the Brookian orogeny in a deep-marine setting (Young, 2004). It overlies, disconformably to unconformably, the Etivluk Group and older rocks. Some parts of the Okpikruak contain large (meter- to outcrop-sized) blocks of various lithologies that have been interpreted as olistoliths (Young, 2004). Okpikruak clasts and blocks reflect a diverse provenance including numerous stratigraphic units and 
allochthons. A single carbonate boulder from this unit (table 9, loc, 62) produced conodonts derived from strata of at least three ages: Mississippian, earliest Early-Middle Pennsylvanian, and middle Early Triassic. The most likely sources for these conodonts are, respectively, the Kogruk, Nuka, and Otuk Formations; details of these correlations are discussed in the sections of this paper devoted to these units.

\section{NUKA FORMATION AND RELATED ROCKS (TABLES 10, 13)}

The Nuka Formation (Carboniferous) consists of medium- to coarse-grained, variably glauconitic limestone, arkosic limestone, and variably arkosic sandstone (Curtis and others, 1984; Young, 2004). It is recognized only in the Bogie plate (sequence) of the NRA (Mayfield and others, 1988), but a somewhat similar unnamed unit (map unit $\mathrm{MDl}_{5}$ of Curtis and others, 1984) occurs in the Bastille plate (sequence) of the NRA (fig. 3). Unit $\mathrm{MDl}_{5}$ (Mississippian and (or) Devonian limestone) consists mainly of locally glauconitic limestone with some interbeds of fine- to coarse-grained feldspathic sandstone.

Studies of conodonts and foraminifers suggest that the depositional age of the Nuka is Late Mississippian-Middle Pennsylvanian (late Meramecian-early Atokan) (Curtis and others, 1984; Mayfield and others, 1984, 1987, 1990; Young, 2004). Only fossils of Devonian age, including Middle-early Late Devonian corals and brachiopods and Late Devonian foraminifers, have been reported from unit $\mathrm{MDl}_{5}\left(\mathrm{Curtis}_{\text {and }}\right.$ others, 1984).

Tables 10 and 13 contain conodont data from the Nuka Formation and unit $\mathrm{MDl}_{5}$ (fig. 7). Two Nuka localities in the Noatak quadrangle yielded faunas of late 
Red Dog Fossils OF 2/24/06 4:55 PM p. 38

Meramecian-early Atokan age (table 13, locs. 163, 165). A Nuka sample from the De

Long Mountains (table 10, loc. 16) produced a more definitive fauna no older than early Middle Pennsylvanian (late Morrowan) in age that also contained redeposited late Late Mississippian (Chesterian) forms. Two samples of skeletal supportstone from unit $\mathrm{MDl}_{5}$ were barren (table 10, locs. 118, 119).

A heterolithic carbonate boulder in the Okpikruak Formation (table 9, loc. 62) produced some conodonts of earliest Early-Middle Pennsylvanian (earliest Morrowanearly Desmoinesian) age. The Nuka Formation is the only carbonate-rich unit in the western Brooks Range that has yielded conodonts of this age and is thus the most likely source for this fauna.

\section{ENDICOTT GROUP (TABLES 11, 13; PLATE 3)}

The Endicott Group is a chiefly siliciclastic succession deposited mainly in shallow-marine to non-marine settings. It comprises, in ascending order, the Hunt Fork Shale, Noatak Sandstone, Kanayut Conglomerate, and Kayak Shale (fig. 2). Much of the Endicott consists of fluvial and marginal marine sandstones and conglomerates that contain few fossils, but marine shales in the Hunt Fork and Kayak include some bioclastic limestone layers. Megafossils (chiefly brachiopods) indicate a Late Devonian age for the Hunt Fork and the Noatak in the western and west-central Brooks Range; the Kanayut is considered Late Devonian-Mississippian(?) (Ellersieck and others, 1984; Karl and others, 1989; Dutro and others, 1994). The Kayak is well-dated as early Early Mississippian (Kinderhookian) in the west-central Brooks Range (e.g., Dumoulin and 
Harris, 1997; Mull and others, 1997) but few definitive age data have been published previously from the De Long Mountains.

Tables 11 and 13 contain 15 samples from 14 locations in the Kayak Shale. Collections are chiefly from the EMA (Key Creek and Wolverine Creek plates) but several are from the PCA (Amaruk plate). Six collections (table 11, locs. 15, 158, 175, 177, 178; table 13, loc. 174) from the Key Creek and Amaruk plates produced diagnostic faunas; all are Kinderhookian, and three are middle-late Kinderhookian (figs. 4, 6). Kayak lithofacies sampled for this report range from skeletal wackestone to grainstone with a variety of bioclasts. Most samples contain notable (10 to 25\%) quartz and other noncarbonate detritus. Conodont biofacies from the EMA suggest shallow-water settings locally characterized by high-energy conditions and restricted circulation. Two samples, one from the Port Road succession of the Key Creek plate (loc. 159) and the other from the Amaruk plate (loc. 15) are phosphatic lag concentrates; the latter was deposited in a middle-shelf or deeper setting. Phosphatic, phosphatized, and (or) pyritic bioclasts are a notable component of heavy-mineral concentrates from most Kayak samples.

Very fine to fine-grained sandstones in the Hunt Fork Shale and Noatak Sandstone (table 11, locs. 90, 105) contained sparse brachiopod fragments but were barren of conodonts. Clasts in both samples are chiefly monocrystalline quartz with subordinate polycrystalline quartz and sedimentary lithoclasts. The Hunt Fork sample also contains mafic volcanic lithoclasts including possible tuff fragments. 
Carbonate strata of Devonian age are found in a number of thrust sheets in the western Brooks Range and consist of locally fossiliferous limestone and dolostone with some siliciclastic interbeds (e.g., Mayfield and others, 1987, 1988). Samples of these rocks in this study come from the EMA, PCA (Amaruk plate), KRA (Amphitheatre, Kelly, and Wulik Peaks plates), and NRA, as well as some strata of uncertain structural position; collections are from the De Long Mountains (table 12, 15 samples from 13 localities) and Noatak (table 13, 12 samples from 11 localities) quadrangles. Many of these rocks have been called Baird Group by previous authors (e.g., Mayfield and others, 1984, 1988) but most differ from the Baird Group in its type area in the Baird Mountains quadrangle in age, metamorphic grade, and stratigraphic and structural position. The type Baird Group is Ordovician to early Middle Devonian (mostly Early Devonian and older), metamorphosed (conodont CAI values $\geq 5$ ), grades upward into DevonianMississippian siliciclastic strata of the Endicott Group, and may be part of the EMA. All of the Devonian carbonate rocks listed in table 12 are unmetamorphosed (CAI 3-4), part of the PCA or KRA, and nowhere contiguous with the type Baird Group, and most are of Middle or Late Devonian age. These De Long Mountains strata are similar to Devonian limestone (map unit Dlk) in the Howard Pass quadrangle that was not included in the Baird Group (Dover and others, 2004). We follow that precedent here for Devonian carbonate rocks in the De Long Mountains quadrangle, and suggest that some coeval carbonate strata in the Noatak quadrangle (table 13, locs. 168, 170, 180-184) should also be excluded from the Baird Group because of their age, metamorphic grade, and (or) stratigraphic, structural, and (or) spatial position. However, other collections in the eastern and southern Noatak quadrangle (table 13, locs. 185-188) come from an outcrop 
belt contiguous (Mayfield and others, 1988), and perhaps correlative, with the type Baird Group.

Eight samples from Devonian carbonate rocks herein excluded from the Baird Group yielded relatively diagnostic conodont collections (fig. 8). The oldest of these (loc. 26) is latest Emsian-early Eifelian (late Early-early Middle Devonian) and the youngest (loc. 104) is Famennian (late Late Devonian). Six collections (locs. 11, 28, 101; table 13, locs. 168, 181, 182) indicate ages within the Eifelian, Givetian, and (or) Frasnian (Middle-early Late Devonian); limestone at localities 181 and 182 may correlate with limestone of the Nakolik River (map unit Dnl) of Karl and others (1989) in the Baird Mountains quadrangle. Other samples produced mostly meager faunas with relatively long ranges; three samples (table 12) were barren. All collections that yielded biofacies data denote a shallow-water setting, and several faunas imply deposition in or near a high-energy environment. Lithofacies of tightly age-constrained samples range from locally fossiliferous carbonate mudstone to dolomitic crinoidal grainstone. Barren and poorly productive collections come chiefly from limestone rich in peloids, calcispheres, and (or) micritized clasts, suggesting a very shallow water depositional setting that may have had restricted circulation. Data are too scarce to infer differences in lithofacies or biofacies in these rocks on the basis of structural position.

Rocks in the Noatak quadrangle that may correlate with the Baird Group are partly dolomitic and contain local black chert nodules; they produced mostly longranging faunas with CAI values of 4 to 5.5. The most tightly dated collection (loc. 185) is of possible Middle Devonian age; another fauna is Late Silurian-Middle Devonian. No detailed lithofacies or biofacies data are available from these rocks. 


\section{THERMAL DATA}

Color changes in conodonts provide data on the thermal history of the rocks that contain them (Epstein et al., 1977). CAI values from the Lisburne Group in the Red Dog area are mostly 2.5-3.5 (tables 1-8), indicating temperatures of at least $100-200^{\circ} \mathrm{C}$ (Watts et al., 1994). Temperature ranges for CAI values were determined from plots of experimental data of Epstein et al. (1977); a CAI value of 3 indicates a temperature range of $120-190^{\circ} \mathrm{C}$. CAI temperatures can be equated to burial depths for a given geothermal gradient; for example, in the Appalachian basin, overburdens of $\sim 3,600-5,500 \mathrm{~m}$ produced CAI values of 3 (Harris et al., 1978). Total stratigraphic thickness of the Pennsylvanian through Lower Cretaceous section overlying Mississippian strata in the Red Dog area was estimated as generally less than 1,500 m (Mayfield et al., 1988). Thus, stratigraphic overburden under normal geothermal gradients (such as that of the Appalachian basin) is not great enough to account for most Lisburne CAI values in the western Brooks Range.

CAI values in Carboniferous and older rocks in the Red Dog area probably reflect tectonic burial and (or) an elevated geothermal gradient. Harris and others (1987) interpreted CAI values in the western parts of the Misheguk Mountain and Baird Mountains quadrangles as related to tectonic burial rather than prethrust burial metamorphism because CAI values in coeval rocks in these areas increase downward through the stack of thrust sheets. CAI values from the Red Dog area show no such simple patterns, however, and are not consistently higher in lower thrust sheets. Instead, CAI values in Carboniferous and Devonian rocks from the Endicott Mountains, Picnic 
Creek, and Kelly River allochthons in the De Long Mountains quadrangle are essentially identical; 90 percent of $\sim 150 \mathrm{CAI}$ values from these strata in all three allochthons are 2.54 , and more than 80 percent are 2.5 to 3.5 (tables $1-8,11,12$ ). Igneous activity appears to have produced some anomalously high CAIs in parts of the Howard Pass area (Dover and others, 2004). It is possible that elevated CAI values in the Lisburne Group of the Red Dog area reflect, at least in part, regional increases in heat flow related to extensional tectonics, local volcanism, and/or mineralization.

CAI values from Carboniferous and Devonian strata in the Noatak quadrangle show a greater range (from 1 to 5.5) than do values from coeval rocks in the De Long Mountains quadrangle, but the Noatak CAI values also are not uniformly higher in lower allochthons (table 13; table 6, locs. 159, 160; table 7, locs. 159, 167; table 8, loc. 172; table 11, locs. 159, 175, 177, 178). For example, both low (1-2.5) and high (4-5) CAI values occur in thrust sheets in the Noatak quadrangle interpreted by Mayfield and others $(1987,1988)$ as representing low (EMA) and high (NRA) structural levels (table 13, locs. $163,173,184$; table 6 , loc. 159). Conodont values in the Noatak quadrangle do appear to reflect a rough geographic gradient. CAI values from all samples southeast of a southwest-northeast trending line that extends from Ipiavik Lagoon to Kikmiksot and Sivukat Mountains (map 5; samples from EMA, EMA? and NRA) are 4-5.5, whereas values northwest of this line are 1-3 (samples from EMA, PCA, KRA, NRA), and most values northwest of the Wulik River are 1-2. Data from the De Long Mountains quadrangle suggest a comparable pattern in that the lowest CAI values $(1.5,2,2-2.5)$ occur chiefly in the northernmost and far west parts of the quadrangle (e.g., map 1; table 1, locs. 91, 92; table 3, loc. 103; table 6, locs. 2, 3, 94, 116; table 7, loc. 9). A similar 
increase in CAI values for a given unit at the same structural level from south to northnorthwest was seen in CAI values from the Howard Pass quadrangle (Dover and others, 2004).

\section{ACKNOWLEDGMENTS}

Our work at Red Dog was part of a cooperative research project between Teck Cominco American Inc. and the U.S. Geological Survey (1998-2001); additional studies in the Red Dog area were carried out as part of the U.S. Geological Survey North Alaska Fluid Flow project (2002-2004). We thank all of the Teck Cominco staff that participated in the joint project, particularly Jeff Clark, Scott Jennings, Adrian King, Tom Krolak, and John Robinson, and our U.S. Geological colleagues, especially Karen Kelley, Dave Leach, and John Slack, for useful discussions and logistic support. We also thank John Kelley and his co-workers Kevin Evans, Jeanine Schmidt, and Kirk Sherwood for providing us with field notes, maps, and information about the samples they collected. Nora Shew and Daniel Grunwald deftly handled the GIS needs of the project.

\section{REFERENCES}

Armstrong, A.K., 1970, Carbonate facies and lithostrotionid corals of the Mississippian Kogruk Formation, De Long Mountains, Northwestern Alaska: U.S. Geological Survey Professional Paper 664, 38 p.

Blodgett, R.B., and Dutro, J.T., Jr., 1992, Stringocephalus (Brachiopoda) from Middle Devonian (Givetian) rocks of the Baird Group, western Brooks Range, Alaska: Oklahoma Geological Survey Bulletin 145, p. 91-111. 
Blome, C. D., 1983, Upper Triassic Capnuchosphaeridae and Capnodocinae from eastern Oregon: Micropaleontology, v. 29, no. 1, p. 11-49.

Blome, C.D., 1984, Upper Triassic radiolarians and radiolarian zonation for western North America: Bulletins of American Paleontology, v. 85, no. 318, 88 p.

Blome, C.D., Reed, K.M., and Harris, A.G., 1998, Radiolarian and conodont biostratigraphy of the type section of the Akmalik Chert (Mississippian), Brooks Range, Alaska, in Gray, J.E., and Riehle, J., eds., Geologic Studies in Alaska by the U.S. Geological Survey, 1996: U.S. Geological Survey Prof. Paper 1595, p. 51-69.

Blome, C.D., Reed, K.M., and Tailleur, I.L., 1988, Radiolarian biostratigraphy of the Otuk Formation in and near the National Petroleum Reserve in Alaska, in Gryc, G., ed., Geology and exploration of the National Petroleum Reserve in Alaska, 1974-1982: U.S. Geological Survey Professional Paper 1399, p. 725-776.

Bodnar, D.A., 1984, Stratigraphy, age, depositional environments and hydrocarbon source rock evaluation of the Otuk Formation, north-central Brooks Range, Alaska: Fairbanks, University of Alaska, M.S. thesis, 232 p.

Carter, E.S., 1993, Biochronology and paleontology of uppermost Triassic (Rhaetian) radiolarians, Queen Charlotte Islands, British Columbia, Canada: Mémoires de Géologie (Lusanne), no 11, 175 p.

Curtis, S.M., Ellersieck, I., Mayfield, C.F., and Tailleur, I.L., 1984, Reconnaissance geologic map of southwestern Misheguk Mountain quadrangle, Alaska: U.S. Geological Survey Miscellaneous Investigations Series Map I-1502, 2 sheets, scale 1:63,360.

Curtis, S.M., Ellersieck, I., Mayfield, C.F., and Tailleur, I.L., 1990, Reconnaissance geologic map of the De Long Mountains A-1 and B-1 quadrangles and part of the C-1 quadrangle, Alaska: 
Red Dog Fossils OF 2/24/06 4:55 PM p. 46

U.S. Geological Survey Miscellaneous Investigations Series Map I-1930, 2 sheets, scale $1: 63,360$.

De Vera, J., McClay, K.R., and King, A.R., 2004, Structure of the Red Dog district, western Brooks Range, Alaska: Economic Geology, v. 99, p.

DeWever, P., 1979, Systematic section, in DeWever, P., Sanfilipo, A., Riedel, W. R., and Gruber, B., Triassic radiolarians from Greece, Sicily, and Turkey: Micropaleontology, v. 25, no. 1, p. 75-109.

Dover, J.H., Tailleur, I.L., and Dumoulin, J.A., 2004, Geologic and fossil locality maps of the west-central part of the Howard Pass quadrangle and part of the adjacent Misheguk Mountain quadrangle, western Brooks Range, Alaska: U.S. Geological Survey Miscellaneous Field Studies Map MF-2413, 2 sheets with 25 p. of explanatory text, scale 1:100,000.

Dumoulin, J.A., and Harris, A.G., 1992, Devonian-Mississippian carbonate sequence in the Maiyumerak Mountains, western Brooks Range, Alaska: U.S. Geological Survey Open-File Report 92-3, 83 p.

Dumoulin, J.A., and Harris, A.G., 1997, Kinderhookian (Lower Mississippian) calcareous rocks of the Howard Pass quadrangle, western Brooks Range, in Dumoulin, J.A., and Gray, J.E., eds., Geologic Studies in Alaska by the U.S. Geological Survey, 1995: U.S. Geological Survey Professional Paper 1574, p. 243-268.

Dumoulin, J.A., Harris, A.G., Blome, C.D., and Young, L.E., 2004, Depositional settings, correlation, and age of Carboniferous rocks in the western Brooks Range, Alaska: Economic Geology, v. 99, p. 1355-1384. 
Dumoulin, J.A., Harris, A.G., and Schmidt, J.M., 1993, Deep-water lithofacies and conodont faunas of the Lisburne Group, western Brooks Range, Alaska, in Dusel-Bacon, C., and Till, A.B., eds., Geologic Studies in Alaska by the U.S. Geological Survey during 1992: U.S. Geological Survey Bulletin 2068, p. 12-30.

Dutro, J.T., Jr., Blodgett, R,B., and Mull, C.G., 1994, Cyrtospirifer from Upper Devonian rocks of the Endicott Group, west-central Brooks Range, Alaska: in Till, A.B., and Moore, T.E., eds., Geologic Studies in Alaska by the U.S. Geological Survey, 1993: U.S. Geological Survey Bulletin 2107, p. 133-142.

Ellersieck, I., Curtis, S.M., Mayfield, C.F., and Tailleur, I.L., 1984, Reconnaissance geologic map of south-central Misheguk Mountain quadrangle, Alaska: U.S. Geological Survey Miscellaneous Investigations Series Map I-1504, 2 sheets, scale 1:63,360.

Ellersieck, I., Curtis, S.M., Mayfield, C.F., and Tailleur, I.L., 1990, Reconnaissance geologic map of the De Long Mountains A-2 and B-2 quadrangles and part of the C-2 quadrangle, Alaska: U.S. Geological Survey Miscellaneous Investigations Series Map I-1931, 2 sheets, scale 1:63,360.

Epstein, A.G., Epstein, J.B., and Harris, L.D., 1977, Conodont color alteration-an index to organic metamorphism: U.S. Geological Survey Professional Paper 995, 27 p.

Gradstein, F.M., Ogg, J.G., and 37 others, 2004, A Geologic Time Scale 2004: Cambridge, Cambridge University Press, 589 p.

Harris, A.G., Harris, L.D., and Epstein, J.B., 1978, CAI and gas data from Paleozoic rocks in the Appalachian basin: maps for assessing hydrocarbon potential and thermal maturity (conodont color alteration isograds and overburden isopachs): U.S. Geological Survey Miscellaneous Investigations Series Map I-917-B, 4 sheets, scale 1:2,500,000. 
Harris, A.G., Lane, H.R., Tailleur, I.L., and Ellersieck, I., 1987, Conodont thermal maturation patterns in Paleozoic and Triassic rocks, northern Alaska-Geologic and exploration implications, in Tailleur, I.L., and Weimer, Paul, eds., Alaskan North Slope Geology: Bakersfield, Calif., Pacific Section, Society of Economic Paleontologists and Mineralogists, Book 50, p. 181-194.

Holdsworth, B.K., and Murchey, B.L., 1988, Paleozoic radiolarian biostratigraphy of the northern Brooks Range, Alaska, in Gryc, G., ed, Geology and Exploration of the National Petroleum Reserve in Alaska, 1974-1982: U.S. Geological Survey Professional Paper 1399, p. 777-792. Ishiga, Hiroaki, 1986, Late Carboniferous and Permian radiolarian biostratigraphy of southwest Japan: Journal of Geosciences, Osaka City University, v. 29, p. 89-100.

Karl, S.M., Dumoulin, J.A., Ellersieck, I., Harris, A.G., and Schmidt, J.M., 1989, Preliminary geologic map of the Baird Mountains and part of the Selawik quadrangles, Alaska: U.S. Geological Survey Open-File Report 89-551, 65 p., 1 pl. scale:1:250,000.

Kelley, K.D., Dumoulin, J.A., and Jennings, Scott, 2004, The Anarraaq Zn-Pb-Ag and barite deposit, northern Alaska; Evidence for replacement of carbonate by barite and sulfides: Economic Geology, v. 99, p.

Mayfield, C.F., Curtis, S.M., Ellersieck, I., and Tailleur, I.L., 1984, Reconnaissance geologic map of southeastern Misheguk Mountain quadrangle, Alaska: U.S. Geological Survey Miscellaneous Investigations Map I-1503, 2 sheets, scale 1:63,360.

Mayfield, C.F., Curtis, S.M., Ellersieck, I., and Tailleur, I.L., 1990, Reconnaissance geologic map of the De Long Mountains A-3 and B-3 quadrangles and parts of the A-4 and B-4 quadrangles, Alaska: U.S. Geological Survey Miscellaneous Investigations Series Map I-1929, 2 sheets, scale $1: 63,360$. 
Mayfield, C.F., Ellersieck, I., and Tailleur, I.L., 1987, Reconnaissance geologic map of the Noatak C-5, D-5, D-6, and D-7 quadrangles, Alaska: U.S. Geological Survey Miscellaneous Investigations Series Map I-1814, 1 sheet, scale 1:63,360.

Mayfield, C.F., Tailleur, I.L., and Ellersieck, I., 1988, Stratigraphy, structure, and palinspastic synthesis of the western Brooks Range, northwestern Alaska, in Gryc, G., ed., Geology and exploration of the National Petroleum Reserve in Alaska, 1974-1982: U.S. Geological Survey Professional Paper 1399, p. 143-186.

Moore, D.W., Young, L.E., Modene, J.S., and Plahuta, J.T., 1986, Geologic setting and genesis of the Red Dog Zinc-Lead-Silver Deposit, western Brooks Range, Alaska: Economic Geology, v. 81, p. 1696-1727.

Mull, C.G., Crowder, R.K., Adams, K.E., Siok, J.P., Bodnar, D.A., Harris, E.E., Alexander, R.R., and Solie, D.N., 1987, Stratigraphy and structural setting of the Picnic Creek allochthon, Killik River quadrangle, central Brooks Range, Alaska, in Tailleur I.L., and Weimer, P., eds., Alaskan North Slope geology: Society of Economic Paleontologists and Mineralogists, Pacific Section, and Alaska Geological Society, Book 50, p. 650-662.

Mull, C.G., Harris, A.G., and Carter, J.L., 1997, Lower Mississippian (Kinderhookian) biostratigraphy and lithostratigraphy of the western Endicott Mountains, Brooks Range, Alaska, in Dumoulin, J.A., and Gray, J.E., eds., Geological studies in Alaska by the U.S. Geological Survey, 1995: U.S. Geological Survey Prof. Paper 1574, p. 221-242.

Mull, C.G., Tailleur, I.L., Mayfield, C.F., Ellersieck, I., Curtis, S., 1982, New upper Paleozoic and Mesozoic stratigraphic units, central and western Brooks Range, Alaska: American Association of Petroleum Geologists Bulletin, v. 66, p. 348-362. 
Murchey, B.L., 1990, Age and depositional setting of siliceous sediments in the upper Paleozoic Havallah sequence near Battle Mountain, Nevada: implications for the paleogeography and structural evolution of the western margin of North America, in Harwood, D.S., and Miller, M.M., eds, Paleozoic and early Mesozoic paleogeographic relations: Sierra Nevada, Klamath Mountains, and related terranes: Geological Society of America Special Paper 255, p. 137-155. Murchey, B.L., Jones, D.L., Holdsworth, B.K., and Wardlaw, B.R., 1988, Distribution patterns of facies, radiolarians, and conodonts in the Mississippian to Jurassic siliceous rocks of the northern Brooks Range, Alaska, in Gryc, G., ed., Geology and exploration of the National Petroleum Reserve in Alaska, 1974-1982: U.S. Geological Survey Professional Paper 1399, p. 697-724.

Pessagno, E.A., Jr., Finch, J.W., and Abbott, P.L., 1979, Upper Triassic Radiolaria from the San Hipolito Formation, Baja California: Micropaleontology, v. 25, no. 2, p. 160-197.

Purnell, M.A. and von Bitter, P.H., 1992, Vogelgnathus Norby and Rexroad (Conodonta): new species from the lower Carboniferous of Atlantic Canada and northern England: Journal of Paleontology, v. 66, no. 2, p. 311-331.

Sable, E.G., and Dutro, J.T., Jr., 1961, New Devonian and Mississippian formations in the De Long Mountains, northern Alaska: American Association of Petroleum Geologists Bulletin, v. 45, p. 585-593.

Slack, J.F., Dumoulin, J.A., Schmidt, J.M., Young, L.E., and Rombach, C.S., 2004, Paleozoic sedimentary rocks in the Red Dog $\mathrm{Zn}-\mathrm{Pb}-\mathrm{Ag}$ district and vicinity, western Brooks Range, Alaska: Provenance, deposition, and metallogenic significance: Economic Geology, v. 99, p. $1385-1414$. 
Watts, K.F., Harris, A.G., Carlson, R.C., Eckstein, M.K., Gruzlovic, P.D., Imm, T.A., Krumhardt, A.P., Lasota, D.K., Morgan, S.K., Enos, Paul, Goldstein, R.H., Dumoulin, J.A., and Mamet, B.L., 1994, Analysis of reservoir heterogeneities due to shallowing-upward cycles in carbonate rocks of the Upper Mississippian and Pennsylvanian Wahoo Limestone of northeastern Alaska: Department of Energy Open-File Report, Contract DE-AC22-89BC14471, 433 p.

Wilson, J.L., 1975, Carbonate Facies in Geologic History: Springer-Verlag, New York, 470 p. Young, L.E., 1992, The Wolverine Creek sequence: evidence for an allochthon below the Brooks Range allochthon, western Brooks Range, Alaska: Alaska Division of Geological and Geophysical Surveys Report of Investigations 92-4, 19 p.

Young, L.E., 2004, A geologic framework for mineralization in the western Brooks Range, Alaska: Economic Geology, v. 99, p. 1281-1306. 


\section{FIGURE CAPTIONS}
Figure 1. Distribution of the Lisburne Group (stippled) in northern Alaska and location of quadrangles and localities mentioned in text (from Dumoulin and others, 2004). PR=Port Road. SR=Spiny Ridge.

Figure 2. Generalized stratigraphic column for the Endicott Mountains allochthon in the western Brooks Range showing principal Paleozoic and Mesozoic formations. Note: in some allochthons, the Utukok Formation directly overlies Devonian carbonate rocks. Unit thicknesses are schematic. Modified from Young (2004).

Figure 3. Structural hierarchy for allochthons and plates in the Red Dog area, based mainly on Young (2004).

Figure 4. Depositional setting, fossil control, and stratigraphic context of Lisburne Group successions in the Red Dog area. Only fossil groups that most narrowly restrict the age of the collection or unit are listed. Data sources: Curtis and others. $(1984,1990)$, Dumoulin and Harris (1992), Ellersieck and others (1990), Mayfield and others (1990), this paper. Absolute ages are from Gradstein and others (2004). Amph.=Amphitheater plate, IR=Ipnavik River allochthon, Nach. Pass=Nachralik Pass plate, $\mathrm{SD}=$ S. anchoralisD. latus conodont zone, Wolv.=Wolverine Creek plate,;11, 12, and 15-18 are Mamet foraminifer zones.

Figure 5. Age range of most biostratigraphically useful conodont and radiolarian collections from deep-water facies of the Lisburne Group; data from tables 1-5, 14 of this report. Locations shown on maps (sheets 1 and 2). Absolute ages are from Gradstein and others (2004). Ik.=Ikalukrok unit, Key Ck.=Key Creek plate, Kiv.=Kivalina unit, Kuna=Kuna Formation, LG=Lisburne Group, Nach. Pass $=$ Nachralik Pass plate, Wolv. $=$ Wolverine Creek plate. ${ }^{*}$, range of older, redeposited 
Red Dog Fossils OF 2/23/06 5:23 PM p. 2

conodonts in samples from locs. 35, 92, and 128 (sample 3). C, R indicates age is based on combined constraints from conodonts and radiolarians.

Figure 6. Age range of most biostratigraphically useful conodont collections from mainly shallow-water facies of the Lisburne Group (Kogruk and Utukok Formations and related strata) and Kayak Shale; data from tables 6-8, 9, 11, 13 of this report. Absolute ages are from Gradstein and others (2004). Dashed part of bar indicates age range less likely based on overall assemblage in this collection or other collections from this unit. A=Amphitheatre plate, Am.=Amaruk plate, $\mathrm{C}=$ Chimney plate, $\mathrm{E}=$ Eli plate, $\mathrm{K}=$ Kelly plate, $\mathrm{KC}=$ Key Creek plate, $\mathrm{PC}=$ Picnic Creek allochthon, $\mathrm{U}=$ plate uncertain, $\mathrm{Wolv} .=\mathrm{Wolverine}$ Creek plate, $\mathrm{WP}=\mathrm{Wulik}$ Peaks plate.

Figure 7. Age range of most biostratigraphically useful radiolarian and conodont collections from the Etivluk Group and Nuka Formation; data from tables 9, 10, 13, and 14 of this report. Dashed part of bar indicates age range less likely based on overall assemblage in this collection. $\mathrm{b}=$ Pantanellium silberlingi subzone of Betraccium deweveri radiolarian zone, $\mathrm{c}=$ Capnodoce radiolarian zone, ,KC=Key Creek plate, $\mathrm{KR}=$ Kelly River allochthon, $\mathrm{PC}=$ Picnic Creek allochthon, $\mathrm{RD}=$ Red Dog plate, $\mathrm{U}=$ plate uncertain, $\mathrm{W}=$ Wulik plate, Wolv. $=$ Wolverine Creek plate, $\mathrm{WP}=\mathrm{Wulik}$ Peaks plate, ${ }^{*}$, range of older, redeposited conodonts in samples from loc. 16.

Figure 8. Age range of most biostratigraphically useful conodont collections from Devonian carbonate rocks; data from tables 12 and 13 of this report. See text for discussion of stratigraphic nomenclature. EM=Endicott Mountains allochthon, $\mathrm{KR}=$ Kelly River allochthon, $\mathrm{PC}=\mathrm{Picnic}$ Creek allochthon, $\mathrm{U}=$ allochthon uncertain. 
Figure 1

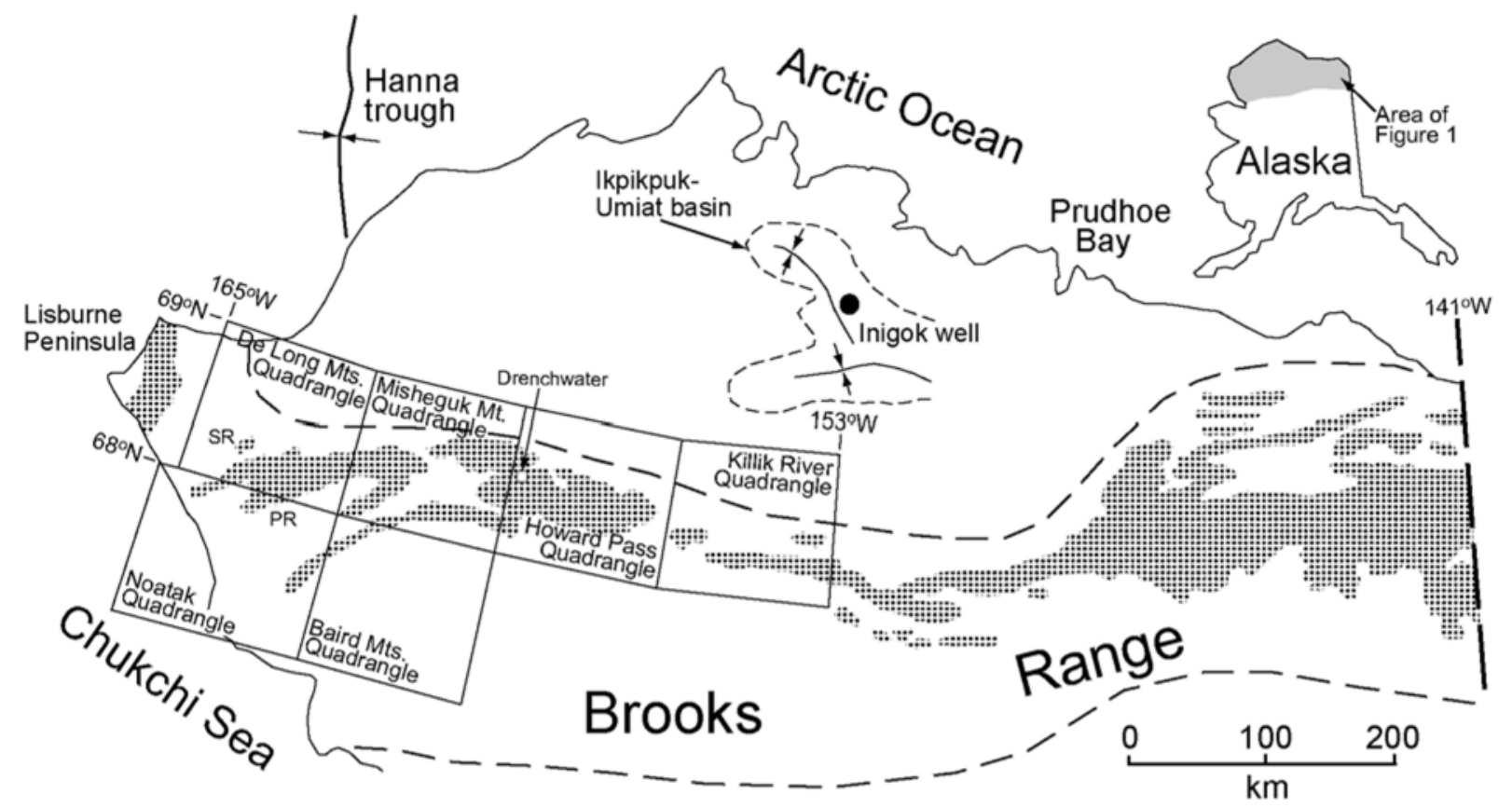


Figure 2

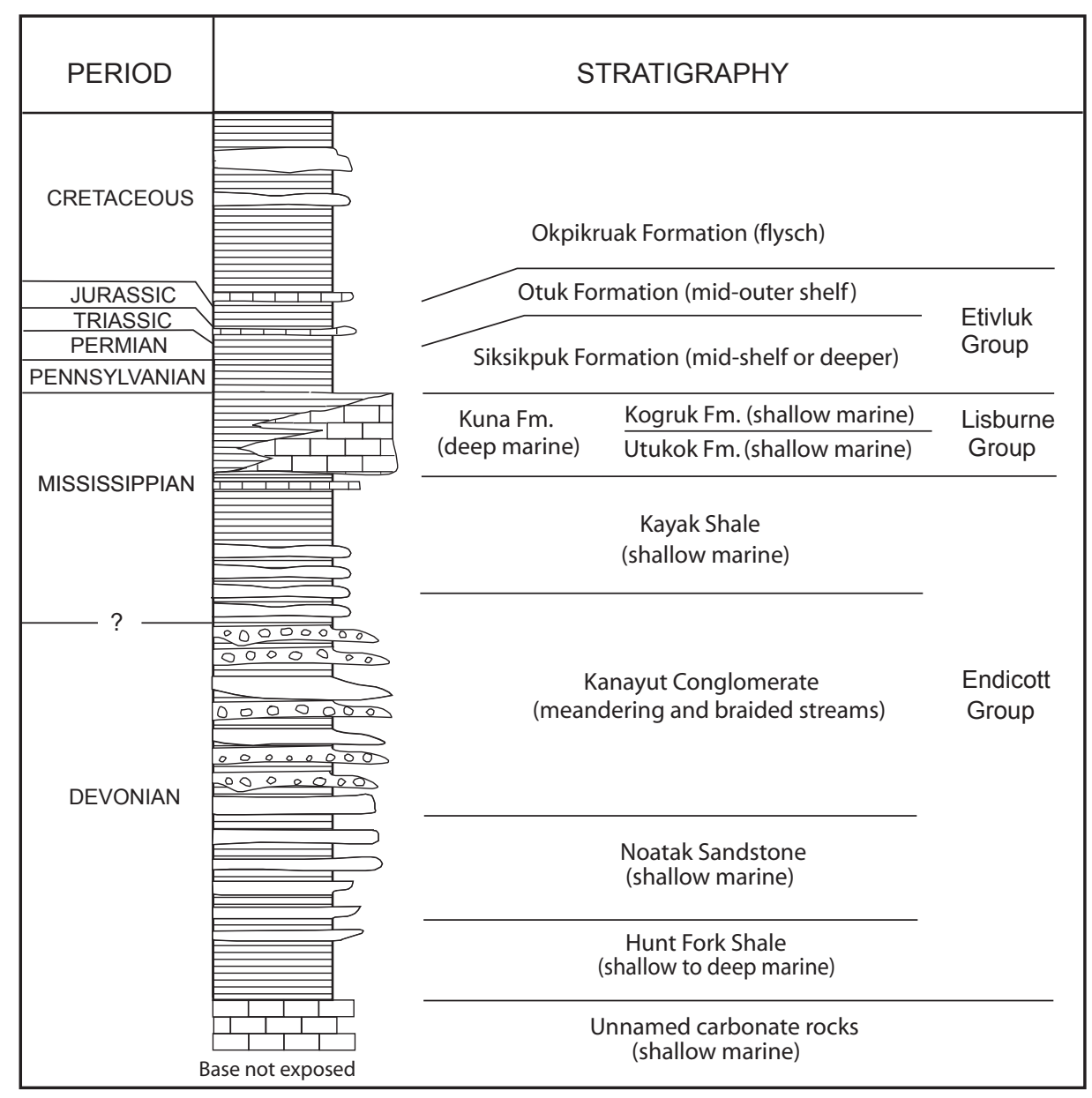


Figure 3

\begin{tabular}{|c|c|}
\hline Allochthon & Plate \\
\hline \multirow{2}{*}{$\begin{array}{c}\text { Nuka } \\
\text { Ridge }\end{array}$} & Bogie \\
\cline { 2 - 2 } Ipnavik River & Bastille \\
\hline \multirow{2}{*}{$\begin{array}{c}\text { Nachralik } \\
\text { Pass } \\
\text { River }\end{array}$} & Eli \\
\cline { 2 - 2 } & $\begin{array}{c}\text { Wulik Peaks- } \\
\text { Kelly }\end{array}$ \\
\cline { 2 - 2 } $\begin{array}{c}\text { Chimney- } \\
\text { Amphitheatre }\end{array}$ \\
\hline \multirow{2}{*}{$\begin{array}{c}\text { Picnic } \\
\text { Creek }\end{array}$} & Amaruk \\
\cline { 2 - 2 } & Wulik \\
\hline \multirow{3}{*}{$\begin{array}{c}\text { Endicott } \\
\text { Mountains }\end{array}$} & Key Creek \\
\cline { 2 - 2 } & Red Dog \\
\cline { 2 - 2 } & Wolverine Creek \\
\hline
\end{tabular}


Figure 4

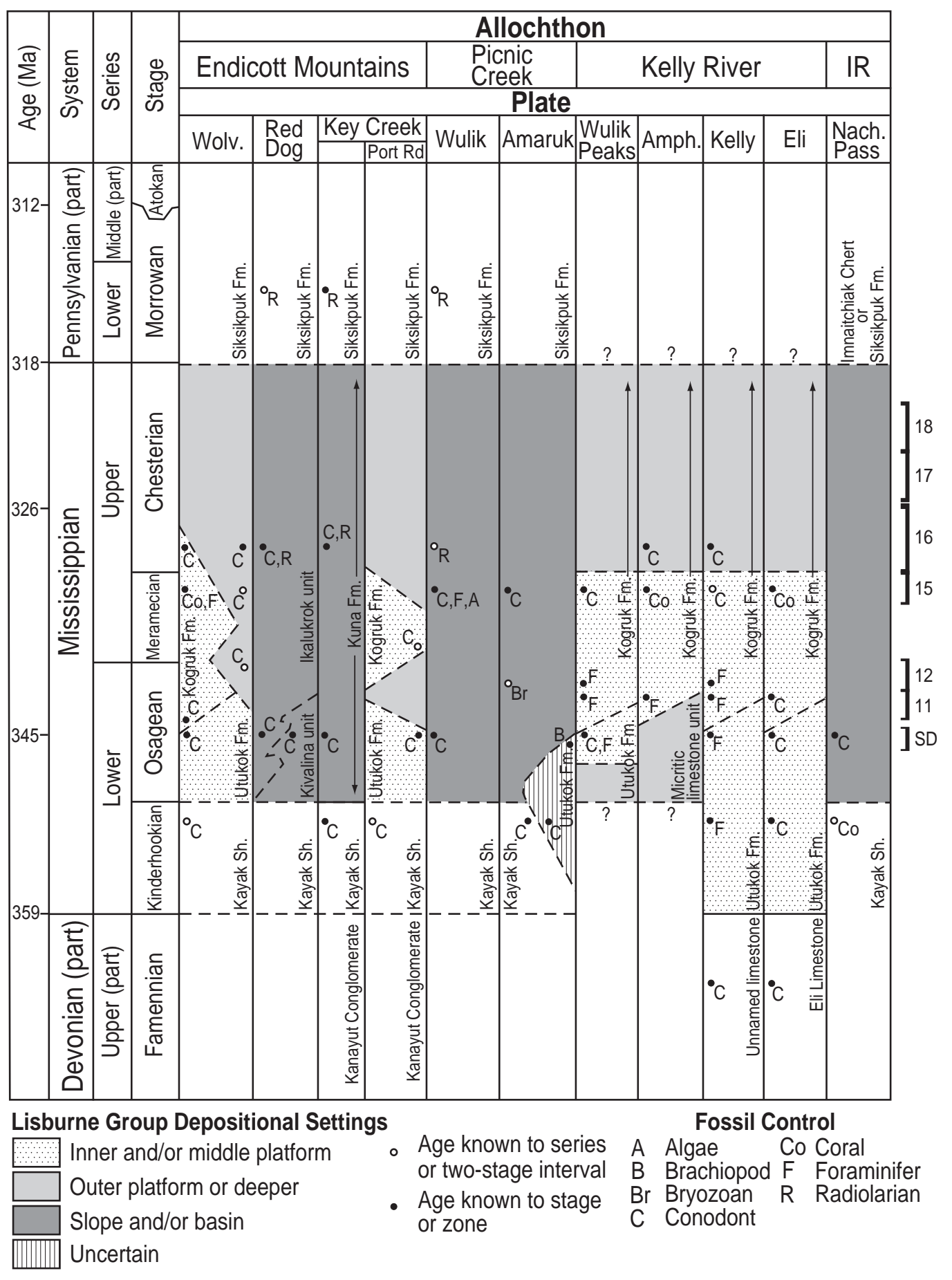


Figure 5

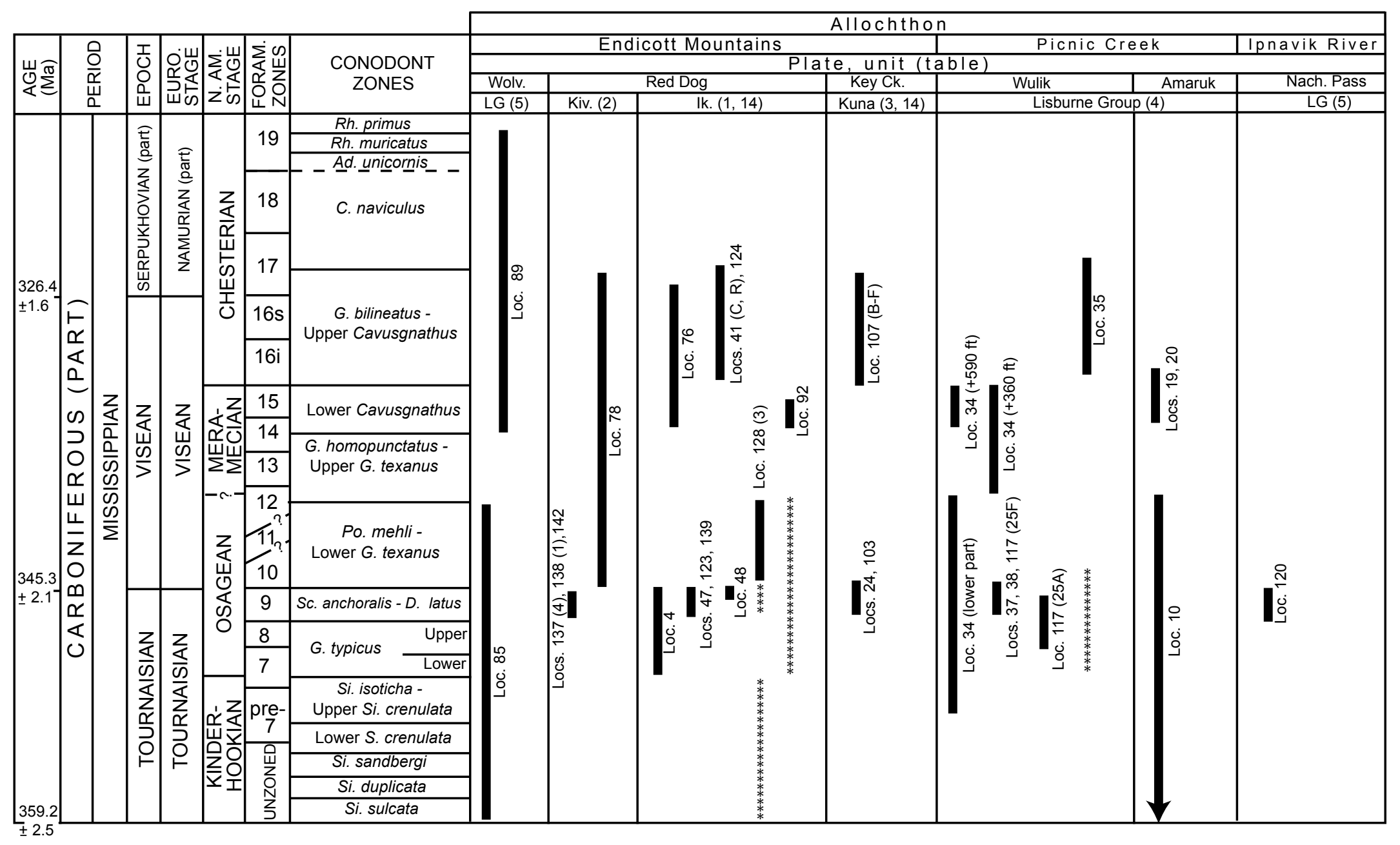


Figure 6

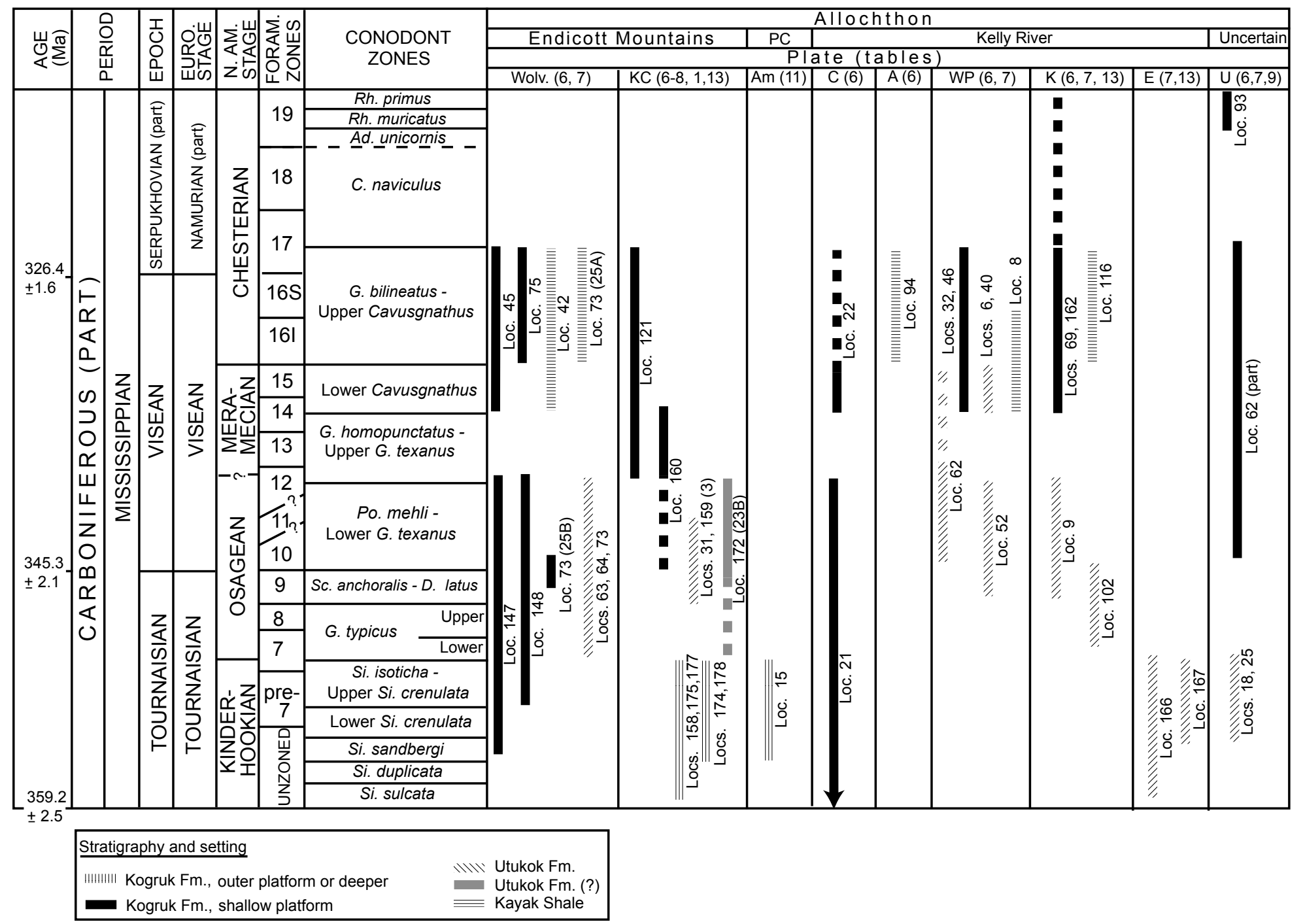


Figure 7

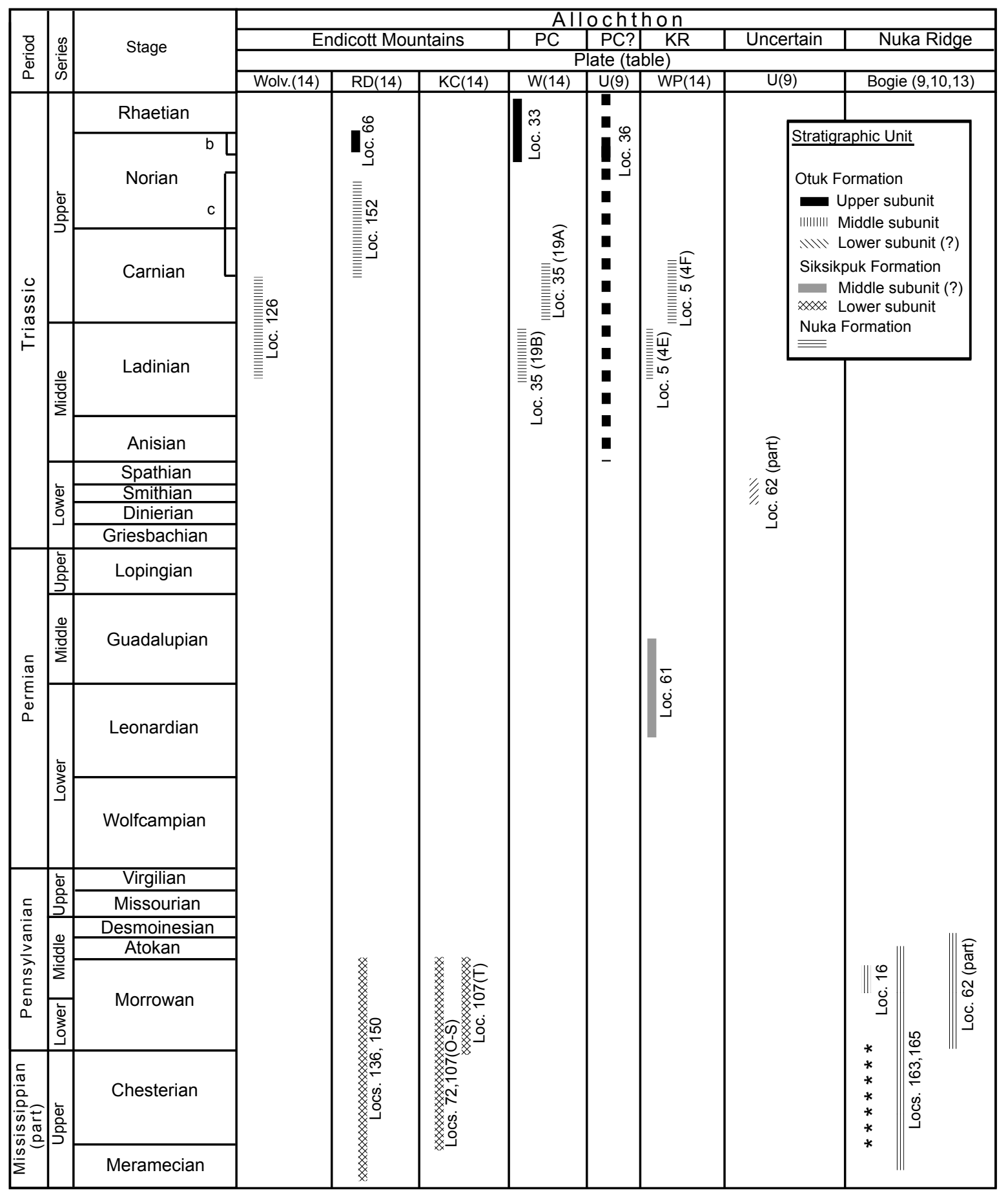


Figure 8

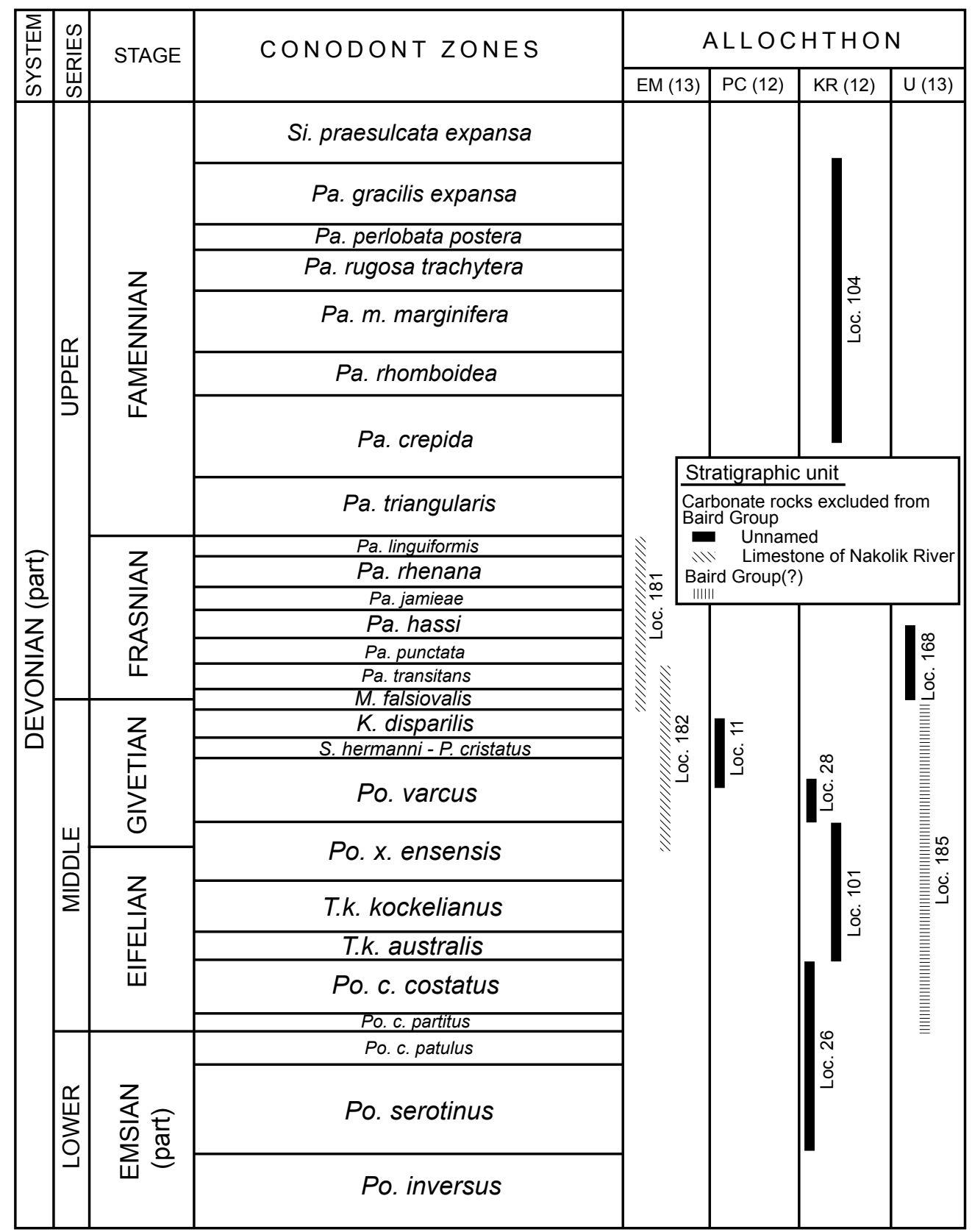


Table 1. Conodont samples from the Ikalukrok unit of the Kuna Formation

[All faunas identified by A.G. Harris. Structural units determined by L.E. Young. EMA, Endicott Mountains allochthon; PCA, Picnic Creek allochthon. CAI, conodont color alteration index. Letters in field number refer to collector: AD, J.A. Dumoulin EK, I. Ellerick; and KE, K. Evans. DDH, dimond drill hole. Interval thicknesses in otherwise indicated. Lithologic data under remarks are field descriptions unless otherwise indicated; thin section observations by J.A. Dumoulin. No., number; indet., indeterminate; loc., locality]

\begin{tabular}{|c|c|c|c|c|c|}
\hline $\begin{array}{l}\text { Locality No., } \\
\text { structural unit }\end{array}$ & $\begin{array}{l}\text { Quadrangle, } \\
\text { latitude n./ } \\
\text { longitude w. }\end{array}$ & $\begin{array}{c}\text { Conodont fauna and CAI } \\
\text { [field No.; USGS collection No.] }\end{array}$ & Age & $\begin{array}{c}\text { Conodont biofacies and } \\
\text { depositional } \\
\text { environment }\end{array}$ & Remarks \\
\hline $\begin{array}{l}4 \\
\text { EMA (Red Dog } \\
\text { plate) }\end{array}$ & $\begin{array}{l}\text { De Long Mtns. } \\
\text { B-3 } \\
68^{\circ} 22^{\prime} 12^{\prime \prime} / \\
163^{\circ} 22^{\prime} 00^{\prime \prime}\end{array}$ & $\begin{array}{l}1 \text { juvenile Pa element Bispathodus utahensis } \\
\text { Sandberg and Gutschick } \\
1 \text { juvenile Pa element Gnathodus sp. indet. } \\
1 \text { S element bar fragment of Scaliognathus } \\
\text { anchoralis Branson and Mehl or its ancestor } \\
22 \text { indet. bar, blade, and platform fragments } \\
\text { CAI=2.5-3 } \\
\text { [79EK189C-2; 27555-PC] }\end{array}$ & $\begin{array}{l}\text { late Early Mississippian } \\
\text { (early-middle Osagean; } G \text {. } \\
\text { typicus Zone-Sc. anchoralis- } \\
\text { Do. latus Zone). }\end{array}$ & $\begin{array}{l}\text { Indeterminate; } \\
\text { conodonts indicate a } \\
\text { distal winnow from or } \\
\text { within a slope or basin } \\
\text { depositional setting. }\end{array}$ & $\begin{array}{l}\text { Sample from a lens of light-gray-weathering limestone } \\
\text { within an interval of shaly limestone. } \\
\text { Fossil loc. 26, Mayfield and others (1990). } \\
\text { Loc. } 7 \text { (app. 1) of Dumoulin and others (2004). }\end{array}$ \\
\hline \multirow[t]{2}{*}{$\begin{array}{l}1 \\
\text { EMA (Red Dog } \\
\text { plate) }\end{array}$} & \multirow[t]{2}{*}{$\begin{array}{l}\text { De Long Mtns. } \\
\text { A-2 } \\
68^{\circ} 14 ' 22^{\prime \prime} / \\
162^{\circ} 54^{\prime} 33.8^{\prime \prime}\end{array}$} & $\begin{array}{l}1 \text { mid Pa element fragment Gnathodus texanus } \\
\text { Roundy? } \\
1 \text { juvenile Pa element Gnathodus sp. indet. of post- } \\
\text { Kinderhookian morphotype } \\
2 \text { Sc elements Gnathodus? sp. indet. } \\
24 \text { indet. bar, blade, and platform fragments } \\
\text { CAI }=\sim 3 \text { (most conodonts covered with organic } \\
\text { matter or very small) } \\
\text { [98AD10A; 33416-PC] }\end{array}$ & $\begin{array}{l}\text { late Early-middle Late } \\
\text { Mississippian (late Osagean- } \\
\text { early Chesterian). } \\
\text { Constraints from } \\
\text { radiolarians at this locality } \\
\text { restrict age to early } \\
\text { Chesterian. }\end{array}$ & $\begin{array}{l}\text { Indeterminate (too few } \\
\text { conodonts); conodonts } \\
\text { present indicate } \\
\text { postmortem transport } \\
\text { within or from a } \\
\text { normal-marine } \\
\text { depositional setting. }\end{array}$ & $\begin{array}{l}\text { Sample from chert-rich upper part of Ikalukrok unit; } \\
\text { see table } 14 \text { for radiolarian sample from this locality. } \\
1 \mathrm{~m} \text { of medium-brown-gray-weathering, dark-gray, } \\
\text { fetid, cross-laminated(?), very fine grained limestone } \\
\text { in } 0.25 \text { to } 1 \mathrm{~cm} \text { thick beds, interbedded with thin- } \\
\text { bedded black siliceous mudstone. Thin section is } \\
\text { packed, calcareous radiolarite with minor barite; test } \\
\text { structure of some radiolarians quite well-preserved. } \\
\text { Heavy-mineral concentrate includes muscovitic and } \\
\text { (or) chloritic schist fragments. } \\
8.3 \mathrm{~kg} \text { of rock was processed. } \\
\text { Loc. } 3 \text { (fig. } 2 \text { ) of Dumoulin and others (2004). }\end{array}$ \\
\hline & & $\begin{array}{l}1 \text { Pa element fragment Gnathodus texanus Roundy } \\
1 \text { unassigned Pb element } \\
9 \text { indet. bar, blade, and platform fragments } \\
\text { CAI }=4 \\
{[8-16-83 \mathrm{C} ; 29229-\mathrm{PC}]}\end{array}$ & $\begin{array}{l}\text { late Early-middle Late } \\
\text { Mississippian (late Osagean- } \\
\text { early Chesterian). }\end{array}$ & $\begin{array}{l}\text { Indeterminate (too few } \\
\text { conodonts). }\end{array}$ & $\begin{array}{l}\text { Lower part of chert-rich interval, upper Ikalukrok unit. } \\
\text { Stratigraphic level probably close to that of } \\
\text { 98AD10A. } \\
\text { Massive-bedded and laminated lenses of chert, } \\
\text { limestone, and dolostone. Sampled dark-gray, very } \\
\text { fine grained limestone that weathers moderate } \\
\text { yellowish brown. Thin section is packed, calcareous } \\
\text { radiolarite with minor barite; test structure of some } \\
\text { radiolarians very well preserved. } \\
\text { 5.0 kg of rock was processed. } \\
\text { Sample collected by A.G. Harris. }\end{array}$ \\
\hline
\end{tabular}


Table 1. Conodont samples from the Ikalukrok unit of the Kuna Formation-Continued.

[All faunas identified by A.G. Harris. Structural units determined by L.E. Young. EMA, Endicott Mountains allochthon; PCA, Picnic Creek allochthon. CAI, conodont color alteration index. Letters in field number refer to collector: AD, J.A. Dumoulin EK, I. Ellersick; and KE, K. Evans. DDH, dimond drill hole. Interval thicknes in drill hos otherwise indicated. Lithologic data under remarks are field descriptions unless otherwise indicated; thin section observations by J.A. Dumoulin. No., number; indet., indeterminate; loc., localityl

\begin{tabular}{|c|c|c|c|c|c|}
\hline $\begin{array}{l}\text { Locality No., } \\
\text { structural unit }\end{array}$ & $\begin{array}{l}\text { Quadrangle, } \\
\text { latitude n./ } \\
\text { longitude w. }\end{array}$ & $\begin{array}{c}\text { Conodont fauna and CAI } \\
\text { [field No.; USGS collection No.] }\end{array}$ & Age & $\begin{array}{c}\text { Conodont biofacies and } \\
\text { depositional } \\
\text { environment }\end{array}$ & Remarks \\
\hline $\begin{array}{l}47 \\
\text { EMA (Red Dog } \\
\text { plate) }\end{array}$ & $\begin{array}{l}\text { De Long Mtns. } \\
\text { A-2 } \\
68^{\circ} 13^{\prime} 10.3^{\prime \prime} / \\
163^{\circ} 3{ }^{\prime \prime} \\
\text { (location } \\
\text { approximate on } \\
\text { long line of } \\
\text { traverse) }\end{array}$ & $\begin{array}{l}19 \text { Pa elements Bispathodus utahensis Sandberg } \\
\text { and Gutschick } \\
1 \mathrm{P} \text { element Eotaphrus sp. indet. } \\
1 \text { juvenile Pa element Polygnathus communis } \\
\text { Branson and Mehl } \\
3 \mathrm{~S} \text { element fragments Scaliognathus anchoralis } \\
\text { Branson and Mehl } \\
\text { Unassigned elements: } \\
9 \mathrm{~Pb} \text { ( } 3 \text { morphotypes), } 2 \mathrm{M} \text { (2 morphotypes), } 1 \mathrm{Sb} \\
\text { and } 6 \mathrm{Sc} \text { (4 morphotypes) } \\
148 \text { indet. bar, blade, and platform fragments } \\
\text { CAI=2.5 } \\
\text { [KE98-17; 33503-PC] }\end{array}$ & $\begin{array}{l}\text { middle late Early } \\
\text { Mississippian (middle } \\
\text { Osagean; Sc. anchoralis-Do. } \\
\text { latus Zone). }\end{array}$ & $\begin{array}{l}\text { Indeterminate (too few } \\
\text { generically identifiable } \\
\text { conodonts. } \\
\text { Off-platform conodont } \\
\text { species and an } \\
\text { abundance of small } \\
\text { delicate conodont } \\
\text { fragments occurring } \\
\text { with radiolarians and } \\
\text { sponge spicules } \\
\text { indicate a slope or } \\
\text { basin depositional } \\
\text { setting. }\end{array}$ & $\begin{array}{l}\text { Concretion, } 20 \times 40 \mathrm{~cm} \text {, in Ikalukrok unit. Brown- } \\
\text { gray-weathering black lime mudstone. Thin section } \\
\text { is calcareous radiolarite with lesser calcareous } \\
\text { sponge spicules; some radiolarians filled with } \\
\text { chalcedony. } \\
\text { Heavy-mineral concentrate includes minor sponge } \\
\text { spicules, pyritized and calcitized radiolarians, and } \\
\text { muscovite. } \\
6.42 \mathrm{~kg} \text { of rock was processed. }\end{array}$ \\
\hline \multirow[t]{2}{*}{$\begin{array}{l}48 \\
\text { EMA (Red Dog } \\
\text { plate) }\end{array}$} & \multirow[t]{2}{*}{\begin{tabular}{|l|} 
De Long Mtns. \\
A-2 \\
$68^{\circ} 12^{\prime} 45.6^{\prime \prime} /$ \\
$163^{\circ} 04^{\prime} 17^{\prime \prime}$ \\
(location \\
approximate on \\
long line of \\
traverse)
\end{tabular}} & $\begin{array}{l}\text { 1 Pa element fragment Bispathodus utahensis } \\
\text { Sandberg and Gutschick } \\
1 \text { Pa element (mostly complete) Gnathodus } \\
\text { cuneiformis Mehl and Thomas } \\
1 \text { Pa element Gnathodus pseudosemiglaber } \\
\text { Thompson and Fellows } \\
1 \mathrm{M} \text { element Kladognathus } \text { sp. indet. } \\
1 \text { Pa element Polygnathus } \text { sp. indet. } \\
63 \text { indet. bar, blade, and platform fragments } \\
\text { CAI=2.5 } \\
\text { [KE98-19A; 33504-PC] }\end{array}$ & $\begin{array}{l}\text { middle late Early } \\
\text { Mississippian (late middle } \\
\text { Osagean; upper half } S c \text {. } \\
\text { anchoralis-Do. latus Zone). }\end{array}$ & $\begin{array}{l}\text { Mixed biofacies; } \\
\text { postmortem transport } \\
\text { from outer shelf or } \\
\text { slope depositional } \\
\text { setting. }\end{array}$ & $\begin{array}{l}\text { Turbidite in Ikalukrok unit. } \\
\text { Medium-gray to medium-dark-gray, fine-grained } \\
\text { limestone. Thin section is sandstone made up of } \\
\text { quartz, crinoid ossicles, noncarbonate mud, and } \\
\text { phosphate clasts. } \\
6.1 \text { kg of rock was processed. }\end{array}$ \\
\hline & & $\begin{array}{l}11 \text { juvenile Pa elements Gnathodus spp. indet. [pl. } \\
1 \text {, fig. } 8] \\
2 \text { Sb-Sc elements Kladognathus sp. } \\
\text { Unassigned elements: } \\
1 \mathrm{M} \text { and } 1 \mathrm{Sc} \\
51 \text { indet. bar, blade, and platform fragments } \\
\text { CAI=2 } \\
\text { [KE98-19B; 33644-PC] }\end{array}$ & $\begin{array}{l}\text { Mississippian, but not very } \\
\text { early Early Mississippian } \\
\text { (late Kinderhookian- } \\
\text { Chesterian). }\end{array}$ & $\begin{array}{l}\text { Indeterminate (too few } \\
\text { conodonts); conodonts } \\
\text { present indicate a } \\
\text { postmortem winnow } \\
\text { into off-shelf or deeper } \\
\text { water depositional } \\
\text { setting. }\end{array}$ & $\begin{array}{l}\text { Concretion, } 10 \times 10 \times 20 \mathrm{~cm} \text {, in Ikalukrok unit. } \\
\text { Partly silicified carbonate, medium-dark-gray to } \\
\text { medium-gray, weathers brown. Thin section is } \\
\text { calcareous radiolarite. Radiolarian test structure } \\
\text { locally well preserved; some tests partly pyritized. } \\
\text { Heavy-mineral concentrate includes minor pyritized } \\
\text { sponge spicules and phosphatic brachiopod } \\
\text { fragments. } \\
2.76 \mathrm{~kg} \text { of rock was processed. }\end{array}$ \\
\hline
\end{tabular}


Table 1. Conodont samples from the Ikalukrok unit of the Kuna Formation - Continued.

[All faunas identified by A.G. Harris. Structural units determined by L.E. Young. EMA, Endicott Mountains allochthon; PCA, Picnic Creek allochthon. CAI, conodont color alteration index. Letters in field number refer to collector: AD, J.A. Dumoulin EK, I Ellersieck; and KE K. Evans. DDH, diamond drill hole. Interval thicknesses in drill holes are drilled hickes otherwise indicated. Lithologic data under remarks are field descriptions unless otherwise indicated; thin section observations by J.A. Dumoulin. No., number; indet., indeterminate; loc., locality]

\begin{tabular}{|c|c|c|c|c|c|}
\hline $\begin{array}{l}\text { Locality No., } \\
\text { structural unit }\end{array}$ & $\begin{array}{l}\text { Quadrangle, } \\
\text { latitude n./ } \\
\text { longitude w. }\end{array}$ & $\begin{array}{c}\text { Conodont fauna and CAI } \\
\text { [field No.; USGS collection No.] }\end{array}$ & Age & $\begin{array}{l}\text { Conodont biofacies and } \\
\text { depositional } \\
\text { environment }\end{array}$ & Remarks \\
\hline \multirow[t]{2}{*}{$\begin{array}{l}49 \\
\text { EMA (Red Dog } \\
\text { plate) }\end{array}$} & \multirow[t]{2}{*}{$\begin{array}{l}\text { De Long Mtns. } \\
\text { A-2 } \\
68^{\circ} 12^{\prime} 12^{\prime \prime} / \\
163^{\circ} 03^{\prime} 48^{\prime \prime}\end{array}$} & \begin{tabular}{|l} 
Barren \\
{$[$ KE98-20A] }
\end{tabular} & & & $\begin{array}{l}\text { Limestone turbidite in Ikalukruk unit, } 21 \mathrm{ft} \text { above } \\
\text { base of } 310 \text {-ft-thick measured section; section may } \\
\text { be faulted and is not stratigraphically complete. } \\
\text { Yellow-brown-weathering, medium-dark-gray, fine- } \\
\text { grained limestone bed ( } \sim 1 \mathrm{ft} \text { thick) in black shale. } \\
\text { Thin section is skeletal packstone; bioclasts mainly } \\
\text { calcareous spicules and rare calcareous radiolarians } \\
\text { in a matrix of noncarbonate mud with rare quartz } \\
\text { silt. } \\
\text { Heavy-mineral concentrate includes rare phosphatic } \\
\text { brachiopod fragments. } \\
3.0 \mathrm{~kg} \text { of rock was processed. }\end{array}$ \\
\hline & & $\begin{array}{l}15 \text { Pa elements (mainly fragments) Bispathodus } \\
\text { utahensis Sandberg and Gutschick } \\
2 \text { juvenile to subadult Pa element fragments } \\
\text { Gnathodus pseudosemiglaber Thompson and } \\
\text { Fellows } \\
1 \text { unassigned M element } \\
74 \text { indet. bar, blade, and platform fragments } \\
\text { CAI=3 } \\
{[\text { KE98-20C; 33505-PC ] }}\end{array}$ & $\begin{array}{l}\text { middle late Early-early Late } \\
\text { Mississippian (middle } S c \text {. } \\
\text { anchoralis-Do. latus Zone } \\
\text { through Meramecian). }\end{array}$ & $\begin{array}{l}\text { Postmortem transport } \\
\text { (winnow) from the } \\
\text { bispathodid biofacies, } \\
\text { suggesting an outer } \\
\text { shelf or deeper water } \\
\text { depositional setting. }\end{array}$ & $\begin{array}{l}\text { Limestone turbidite in Ikalukruk unit, } \sim 123 \mathrm{ft} \text { above } \\
\text { base of measured section. } \\
\text { Brownish-gray limestone bed ( } \sim 1 \mathrm{ft} \mathrm{thick)} \mathrm{underlain} \\
\text { and overlain by black shale and siliceous mudstone. } \\
\text { Thin section is fine-grained skeletal grainstone; } \\
\text { bioclasts mostly crinoid ossicles, lesser ostracodes, } \\
\text { calcareous and siliceous spicules, productid spines, } \\
\text { and bryozoan fragments. } \\
2.1 \mathrm{~kg} \text { of rock was processed. }\end{array}$ \\
\hline $\begin{array}{l}50 \\
\text { EMA (Red Dog } \\
\text { plate) }\end{array}$ & $\begin{array}{l}\text { De Long Mtns. } \\
\text { A-2 } \\
68^{\circ} 11^{\prime} 48^{\prime \prime} / \\
163^{\circ} 03^{\prime} 00^{\prime \prime}\end{array}$ & $\begin{array}{l}1 \text { juvenile Pa element Bispathodus utahensis } \\
\text { Sandberg and Gutschick } \\
3 \text { Pa element fragments Gnathodus texanus Roundy } \\
1 \text { juvenile Pa element fragment Gnathodus sp. } \\
\text { indet. } \\
1 \text { Sc element fragment Kladognathus sp. indet. } \\
100 \text { indet. bar, blade, and platform fragments } \\
\text { CAI=3-3.5 } \\
\text { [8-16-83E; 29231-PC] }\end{array}$ & $\begin{array}{l}\text { very late Early-middle Late } \\
\text { Mississippian (late Osagean- } \\
\text { early Chesterian, but } \\
\text { probably no younger than } \\
\text { Meramecian; P. mehli-lower } \\
\text { G. texanus Zone through at } \\
\text { least Lower Cavusgnathus } \\
\text { Zone) }\end{array}$ & $\begin{array}{l}\text { Indeterminate (too few } \\
\text { conodonts). Because } \\
\text { virtually all conodonts } \\
\text { are relatively small } \\
\text { fragments, this } \\
\text { collection has } \\
\text { undergone } \\
\text { considerable } \\
\text { postmortem transport. }\end{array}$ & $\begin{array}{l}\text { Cycles of sandy, thick-bedded fine-grained limestone } \\
\text { grading up into very dark gray to black argillaceous } \\
\text { limestone, and, finally, to carbonaceous shale. } \\
\text { Collection from near top of turbiditic coarse bed of } \\
\text { medium-dark-gray, medium-gray- to grayish- } \\
\text { orange-weathering, very fine grained silty limestone. } \\
\text { Thin section is fine- to coarse-grained sandstone; } \\
\text { clasts mostly calcareous and (or) pyritized and } \\
\text { include abundant (>20\%) radiolarians and lesser } \\
\text { spicules. Noncarbonate grains } ~ 20 \% \text {, mostly quartz, } \\
\text { minor white mica, and phosphate. } \\
6.0 \text { kg of rock was processed. } \\
\text { Sample collected by A.G. Harris. } \\
\text { Loc. } 10 \text { (fig. } 2 \text { ) of Dumoulin and others (2004). }\end{array}$ \\
\hline
\end{tabular}


Table 1. Conodont samples from the Ikalukrok unit of the Kuna Formation - Continued.

[All faunas identified by A.G. Harris. Structural units determined by L.E. Young. EMA, Endicott Mountains allochthon; PCA, Picnic Creek allochthon. CAI, conodont color alteration index. Letters in field number refer to collector: AD, J.A. Dumoulin EK, I. Ellerick; a KE, K. Evans. DDH, dimond drill hole. Interval thicknesses in drill holes are drilled thickes otherwise indicated. Lithologic data under remarks are field descriptions unless otherwise indicated; thin section observations by J.A. Dumoulin. No., number; indet., indeterminate; loc., locality]

\begin{tabular}{|c|c|c|c|c|c|}
\hline $\begin{array}{l}\text { Locality No., } \\
\text { structural unit }\end{array}$ & $\begin{array}{l}\text { Quadrangle, } \\
\text { latitude n./ } \\
\text { longitude w. }\end{array}$ & $\begin{array}{c}\text { Conodont fauna and CAI } \\
\text { [field No.; USGS collection No.] }\end{array}$ & Age & \begin{tabular}{|c|}
$\begin{array}{c}\text { Conodont biofacies and } \\
\text { depositional } \\
\text { environment }\end{array}$ \\
\end{tabular} & Remarks \\
\hline \multirow[t]{2}{*}{$\begin{array}{l}76 \\
\text { EMA (Red Dog } \\
\text { plate) }\end{array}$} & \multirow[t]{2}{*}{$\begin{array}{l}\text { De Long Mtns. } \\
\text { A-2 } \\
68^{\circ} 04^{\prime} 53.8^{\prime \prime} / \\
163^{\circ} 05^{\prime} 52.6^{\prime \prime}\end{array}$} & $\begin{array}{l}3 \text { Pa elements Cavusgnathus unicornis Youngquist } \\
\text { and Miller [pl. 1, fig. 6] } \\
\text { Gnathodus texanus Roundy (juveniles and adults): } \\
9 \mathrm{~Pa} \text { and } 2 \mathrm{M} \text { elements [pl. 1, fig. 5] } \\
6 \text { element fragments } \text { Idioprioniodus } \text { sp. indet. } \\
235 \text { indet. bar, blade, and platform fragments } \\
\text { CAI=3.5-4 } \\
\text { [99AD11BB; 33479-PC] }\end{array}$ & $\begin{array}{l}\text { middle late Mississippian } \\
\text { (late Meramecian-early, } \\
\text { probably earliest Chesterian) }\end{array}$ & $\begin{array}{l}\text { Indeterminate (too few } \\
\text { generically identifiable } \\
\text { conodonts); likely } \\
\text { postmortem transport } \\
\text { from or within the } \\
\text { gnathodid biofacies } \\
\text { (slope or toe of slope } \\
\text { depositional setting). }\end{array}$ & $\begin{array}{l}\text { Interval of limestone (turbidites?) }>10 \mathrm{~m} \text { thick; sample } \\
\text { from middle of good outcrop. Massive, medium- } \\
\text { gray- (slightly brownish) weathering, black, fine- to } \\
\text { medium-crystalline limestone that has parallel } \\
\text { lamination and is fetid. Thin section is anhedral } \\
\text { calcite crystal mosaic with rare calcite-replaced } \\
\text { radiolarians. } \\
\text { L. Young suggests this limestone interval is } \sim 40 \mathrm{~m} \\
\text { thick. Limestone float found } \sim 100 \mathrm{ft} \text { uphill supports } \\
\text { idea of a thick carbonate section here, but main } \\
\text { carbonate exposure is } 10 \mathrm{~m} \text { thick. } \\
+7.5 \mathrm{~kg} \text { of rock was processed. } \\
\end{array}$ \\
\hline & & $\begin{array}{l}\text { Barren } \\
{[99 \mathrm{AD} 11 \mathrm{~F}]}\end{array}$ & & & $\begin{array}{l}\text { Sample from limestone bed just below base of the } \\
\text { layer sampled in 11BB; a grayish shale break } \\
\text { separates the two carbonate intervals. } \\
\text { 1-m-thick interval of medium-gray- (slightly brownish) } \\
\text { weathering, grayish-black, medium crystalline } \\
\text { limestone in } 2 \text { to } 10 \mathrm{~cm} \text { thick uneven beds. Thin } \\
\text { section is calcareous radiolarite. } \\
\text { Heavy-mineral concentrate is chiefly composite rock } \\
\text { fragments of recrystallized barite(?), with organic } \\
\text { matter, and trace sphalerite(?). } \\
+9.0 \mathrm{~kg} \text { of rock was processed. } \\
\end{array}$ \\
\hline $\begin{array}{l}77 \\
\text { EMA (Red Dog } \\
\text { plate) }\end{array}$ & $\begin{array}{l}\text { De Long Mtns. } \\
\text { A-2 } \\
68^{\circ} 03^{\prime} 37.3^{\prime \prime} / \\
163^{\circ} 08^{\prime} 2.6^{\prime \prime}\end{array}$ & $\begin{array}{l}1 \text { juvenile Pa element Lochriea sp. }-L \text {. cf. } L \text {. } \\
\text { commutata (Branson and Mehl) } \\
1 \text { indet. blade fragment } \\
\text { CAI }=2.5-3 \\
\text { [79EK15B-2; 27505-PC] }\end{array}$ & Late Mississippian & $\begin{array}{l}\text { Indeterminate (too few } \\
\text { conodonts). }\end{array}$ & $\begin{array}{l}\text { Limestone bed about } 20 \mathrm{~m} \text { below top of Kuna } \\
\text { Formation (Ikalukrok unit). } \\
4 \mathrm{~kg} \text { of rock was processed. } \\
\text { Fossil loc. 16, Ellersieck and others (1990). }\end{array}$ \\
\hline
\end{tabular}


Table 1. Conodont samples from the Ikalukrok unit of the Kuna Formation - Continued.

[All faunas identified by A.G. Harris. Structural units determined by L.E. Young. EMA, Endicott Mountains allochthon; PCA, Picnic Creek allochthon. CAI, conodont color alteration index. Letters in field number refer to collector: AD, J.A. Dumoulin EK, I. Ellerick; and KE, K. Evans. DDH, ding otherwise indicated. Lithologic data under remarks are field descriptions unless otherwise indicated; thin section observations by J.A. Dumoulin. No., number; indet., indeterminate; loc., locality]

\begin{tabular}{|c|c|c|c|c|c|}
\hline $\begin{array}{l}\text { Locality No., } \\
\text { structural unit }\end{array}$ & $\begin{array}{l}\text { Quadrangle, } \\
\text { latitude n./ } \\
\text { longitude w. }\end{array}$ & $\begin{array}{c}\text { Conodont fauna and CAI } \\
\text { [field No.; USGS collection No.] }\end{array}$ & Age & \begin{tabular}{|c|} 
Conodont biofacies and \\
depositional \\
environment
\end{tabular} & Remarks \\
\hline \begin{tabular}{|l|}
91 \\
Uncertain; \\
EMA (Red Dog \\
plate) or PCA
\end{tabular} & \begin{tabular}{|l|} 
De Long Mtns. \\
C-1 \\
$68^{\circ} 30^{\prime} 28^{\prime \prime} /$ \\
$162^{\circ} 14^{\prime} 12.2^{\prime \prime}$
\end{tabular} & $\begin{array}{l}35 \text { Pa elements Bispathodus utahensis Sandberg } \\
\text { and Gutschick (most are large and incomplete) } \\
3 \text { incomplete Pa elements Cavusgnathus sp. } \\
20 \text { Pa (mostly incomplete) elements Gnathodus } \\
\text { pseudosemiglaber Thompson and Fellows } \\
1 \text { Pa element Gnathodus texanus Roundy } \\
5 \text { Idioprioniodus sp. indet. element fragments } \\
1 \text { Sa element fragment Kladognathus sp. } \\
3 \text { Pa elements (2 posterior platform fragments and } \\
1 \text { complete Pa (juveniles and } 1 \text { adult ) } \\
\text { Mestognathus beckmanni Bischoff or } M \text {. } \\
\text { praebeckmanni von Bitter, Sandberg, and Orchard } \\
1 \text { Pa element fragment Pseudopolygnathus sp. } \\
\text { indet. } \\
\text { Unassigned elements: } \\
1 \text { Pb, 5 M (4 morphotypes), and } 1 \text { Sa } \\
754 \text { indet. bar, blade, and platform fragmen s } \\
\text { CAI=2.0-2.5 } \\
\text { [03AD28A; 33759-PC] }\end{array}$ & $\begin{array}{l}\text { late Early-middle Late } \\
\text { Mississippian (late middle } \\
\text { Osagean-very early } \\
\text { Chesterian; Po. mehli-Lower } \\
\text { Gn. texanus Zone into } \\
\text { earliest Chesterian). }\end{array}$ & \begin{tabular}{|l} 
Conodonts are \\
hydraulically \\
transported and include \\
normal-marine shelf \\
and slope facies \\
elements as well as \\
very shallow water \\
genus Mestognathus.
\end{tabular} & $\begin{array}{l}\text { Kuna Formation (Ikalukrok unit?); sample taken } \sim 6 \mathrm{~m} \\
\text { below top of partial section of siliceous mudstone } \\
\text { that is } \sim 16 \mathrm{~m} \text { thick. Kuna here overlain (apparently } \\
\text { depositionally) by Okpikruak Formation, but contact } \\
\text { is not exposed. Underlying this mudstone section is } \\
\text { black sooty shale (Ikalukrok?), and then rubble of } \\
\text { black siliceous shale and orange-weathering } \\
\text { calcareous siltstone (Kivalina unit?). } \\
\text { Medium- to medium-light-gray (slightly brownish) } \\
\text { limestone turbidite, weathers very pale yellow } \\
\text { brown. Limy interval is } \sim 40 \text { cm thick; underlain and } \\
\text { overlain mostly by black siliceous mudstone and } \\
\text { chert. Limestone in irregular 5- to 10-cm-thick beds, } \\
\text { with black mud chips and small bioclasts. Thin } \\
\text { section is mostly fine-grained calcite with 10-30\% } \\
\text { dolomite, 5-10\% largely silica-replaced bioclasts } \\
\text { (crinoids, ostracodes, bryozoans, and radiolarians), } \\
5-10 \% \text { black mud clasts, and minor quartz silt. } \\
\text { Heavy-mineral concentrate is chiefly composite, } \\
\text { slightly carbonaceous, phosphatic carbonate grains, } \\
\text { white to translucent fluorite(?), and rare glauconite, } \\
\text { phosphatic brachiopod fragments and pelmatozoan } \\
\text { ossicles. } \\
10.1 \text { kg of rock was processed. }\end{array}$ \\
\hline
\end{tabular}


Table 1. Conodont samples from the Ikalukrok unit of the Kuna Formation - Continued.

[All faunas identified by A.G. Harris. Structural units determined by L.E. Young. EMA, Endicott Mountains allochthon; PCA, Picnic Creek allochthon. CAI, conodont color alteration index. Letters in field number refer to collector: AD, J.A. Dumoulin EK, I. Ellerick; a. KE, K. Evas. DDH, diumo otherwise indicated. Lithologic data under remarks are field descriptions unless otherwise indicated; thin section observations by J.A. Dumoulin. No., number; indet., indeterminate; loc., locality]

\begin{tabular}{|c|c|c|c|c|c|}
\hline $\begin{array}{l}\text { Locality No., } \\
\text { structural unit }\end{array}$ & $\begin{array}{l}\text { Quadrangle, } \\
\text { latitude n./ } \\
\text { longitude w. }\end{array}$ & $\begin{array}{c}\text { Conodont fauna and CAI } \\
\text { (field No.; USGS collection No.) }\end{array}$ & Age & \begin{tabular}{|c|} 
Conodont biofacies and \\
depositional \\
environment
\end{tabular} & Remarks \\
\hline $\begin{array}{l}92 \\
\text { Uncertain; } \\
\text { EMA (Red Dog } \\
\text { plate) or PCA }\end{array}$ & \begin{tabular}{|l|} 
De Long Mtns. \\
C-1 \\
$68^{\circ} 30^{\prime} 20^{\prime \prime} /$ \\
$162^{\circ} 14^{\prime} 9.5^{\prime \prime}$
\end{tabular} & $\begin{array}{l}\text { Bispathodus utahensis Sandberg and Gutschick } \\
144 \text { (mostly incomplete) Pa and } 1 \text { Sb elements } \\
15 \text { complete to incomplete Pa elements } \\
\text { Cavusgnathus unicornis Youngquist and Miller } \\
40 \text { Pa elements (nearly complete to mostly } \\
\text { incomplete) Embsaygnathus asymmetricus } \\
\text { Metcalfe } \\
\text { Idioprioniodus? sp. indet.: } \\
26 \mathrm{~Pa}, 12 \mathrm{~Pb}, 27 \mathrm{M}, 1 \mathrm{Sa}, 9 \mathrm{Sb} \text {, and } 4 \text { Sc elements } \\
\text { and } 24 \text { bar fragments } \\
\text { Kladognathus sp. indet.: } \\
1 \mathrm{M}, 1 \mathrm{Sa} \text {, and } 3 \text { Sb-Sc elements } \\
7 \text { subadult Pa elements (all somewhat incomplete) } \\
\text { Mestognathus beckmanni Bischoff } \\
\text { Redeposited Early Mississippian conodonts: } \\
1 \text { Pa element Gnathodus cuneiformis Mehl and } \\
\text { Thomas } \\
3 \text { Pa element fragments Pseudopolygnathus sp. } \\
\text { indet. } \\
1 \text { bar fragment Scaliognathus anchoralis Branson } \\
\text { and Mehl } \\
\text { Unassigned elements: } \\
7 \text { Pb (6 morphotypes), } 1 \mathrm{M}, 2 \text { Sc } \\
1202 \text { indet. bar, blade, and platform fragments } \\
\text { CAI=2.0-2.5 } \\
\text { [03AD28H; } 33760 \text {-PC] }\end{array}$ & $\begin{array}{l}\text { middle Late Mississippian } \\
\text { (early late Meramecian). } \\
\text { The occurrence of } \\
\text { Cavusgnathus unicornis } \\
\text { with what is now the largest } \\
\text { single collection of the rare } \\
\text { conodont Embsaygnathus } \\
\text { asymmetricus (known only } \\
\text { from northern England, } \\
\text { Dublin County of eastern } \\
\text { Ireland, and northern } \\
\text { Alaska) restricts the age of } \\
\text { this collection to the early } \\
\text { late Meramecian. In most } \\
\text { known occurrences, E. } \\
\text { asymmetricus occurs with } \\
\text { other shallow-water species } \\
\text { (e.g., mestognathids and } \\
\text { cavusgnathids) in the coarser } \\
\text { layers of turbiditic beds. } \\
\text { This collection also contains } \\
\text { relatively rare redeposited } \\
\text { Osagean conodonts. The } \\
\text { most abundant conodont } \\
\text { species in the collection (Bi. } \\
\text { utahensis) could be } \\
\text { indigenous as well as } \\
\text { redeposited. }\end{array}$ & \begin{tabular}{|l} 
Hydraulically \\
redeposited conodonts. \\
Virtually all specimens \\
are incomplete and \\
most are relatively \\
large (40- to 60-mesh \\
sieve fraction). \\
Conodonts are chiefly \\
surface swimmers (Bi. \\
utahensis is a globally \\
widespread eurytopic \\
species) and shallow- \\
water forms \\
(mestognathids \\
occupied one of the \\
shallowest warm-water \\
conodont biofacies). \\
The form species \\
Geniculatus claviger is \\
most likely the Pb \\
element of \\
Embsaygnathus? sp.
\end{tabular} & $\begin{array}{l}\text { Kuna Formation (Ikalukrok unit?). These rocks could } \\
\text { be part of a structural repeat of the Kuna section } \\
\text { sampled in 03AD28A, but the limy interval here is } \\
\text { thicker and contains thicker, more calcareous beds. } \\
\text { However, the limy beds are turbidites, and thus } \\
\text { could be irregularly distributed. Alternately (less } \\
\text { likely), this could be an interval of Lisburne Group } \\
\text { of the PCA in structural contact with Kuna of the } \\
\text { EMA. } \\
\text { Thirty-cm-thick bed of coarse-grained skeletal } \\
\text { grainstone/packstone; weathers light brownish gray } \\
\text { to medium light gray (fresh color is very slightly } \\
\text { darker). Contains abundant crinoid ossicles and } \\
\text { brachiopods to } 1.5 \text { cm in diameter. Thin section is } \\
\text { pelmatozoan grainstone, with abundant and diverse } \\
\text { bryozoans, brachiopods, and ostracodes, minor } \\
\text { foraminifers, algae, and phosphatic bioclasts, and a } \\
\text { few carbonate clasts (one contains calcareous } \\
\text { spicules, another has calcite-replaced radiolarians). } \\
\text { Heavy-mineral concentrate is chiefly phosphatized and } \\
\text { lesser dolomitized rock fragments, phosphatic and } \\
\text { phosphatized bioclasts (mainly conodonts, minor } \\
\text { phosphatic brachiopod fragments, and rare } \\
\text { phosphatized pelmatozoan ossicles and gastropod } \\
\text { steinkerns), and fluoritic carbonate grains. } \\
10.9 \text { kg of rock was processed. }\end{array}$ \\
\hline
\end{tabular}


Table 1. Conodont samples from the Ikalukrok unit of the Kuna Formation - Continued.

[All faunas identified by A.G. Harris. Structural units determined by L.E. Young. EMA, Endicott Mountains allochthon; PCA, Picnic Creek allochthon. CAI, conodont color alteration index. Letters in field number refer to collector: AD, J.A. Dumoulin EK, I. Ellersick; a A. KE, K. Evans. DDH, dimond drill hole. Interval thicknesses in drill holes are drilled thickes otherwise indicated. Lithologic data under remarks are field descriptions unless otherwise indicated; thin section observations by J.A. Dumoulin. No., number; indet., indeterminate; loc., localityl

\begin{tabular}{|c|c|c|c|c|c|}
\hline $\begin{array}{l}\text { Locality No., } \\
\text { structural unit }\end{array}$ & $\begin{array}{l}\text { Quadrangle, } \\
\text { latitude } \mathrm{n} . / \\
\text { longitude w. }\end{array}$ & $\begin{array}{c}\text { Conodont fauna and CAI } \\
\text { [field No.; USGS collection No.] }\end{array}$ & Age & \begin{tabular}{|c|} 
Conodont biofacies and \\
depositional \\
environment
\end{tabular} & Remarks \\
\hline $\begin{array}{l}123 \\
\text { EMA (Red Dog } \\
\text { plate) }\end{array}$ & $\begin{array}{l}\text { De Long Mtns. } \\
\text { A-3 } \\
68^{\circ} 09^{\prime} 45.4^{\prime \prime} / \\
163^{\circ} 12^{\prime} 30.7^{\prime \prime}\end{array}$ & $\begin{array}{l}2 \text { Pa elements Gnathodus cuneiformis Mehl and } \\
\text { Thomas [pl. 1, fig. 1] } \\
1 \text { Pa element Gnathodus sp. indet. } \\
1 \text { Pa element Eotaphrus? cf. E. bultyncki } \\
\text { (Groessens) } \\
1 \text { Pa element Polygnathus communis Branson and } \\
\text { Mehl } \\
60 \text { indet. bar, blade, and platform fragments } \\
\text { CAI=4 } \\
\text { [DDH Su } 31 \text { (Su deposit), 1667-1686 ft; 29280-PC] }\end{array}$ & \begin{tabular}{|l} 
late Early Mississippian \\
(middle Osagean; Sc. \\
anchoralis-Do. latus Zone).
\end{tabular} & $\begin{array}{l}\text { Indeterminate (too few } \\
\text { conodonts); the } \\
\text { diversity indicates } \\
\text { open-marine } \\
\text { conditions. }\end{array}$ & $\begin{array}{l}\text { Sample } \sim 101 \mathrm{ft} \text { below conformable(?) contact of } \\
\text { Siksikpuk Formation and Ikalukrok unit and } \sim 12 \mathrm{ft} \\
\text { above mineralized sulfide zone at Su deposit. } \\
\text { Samples lower half of } 48 \text {-ft-thick interval of } \\
\text { calcareous turbidites with shale interbeds as much as } \\
3 \mathrm{ft} \text { thick. See table } 2 \text { for Kivalina unit sample from } \\
\text { this drill hole. } \\
8.2 \mathrm{~kg} \text { of rock was processed. } \\
\text { Sample collected by D. Moore, I.L. Tailleur, and L.E. } \\
\text { Young. }\end{array}$ \\
\hline $\begin{array}{l}124 \\
\text { EMA (Red Dog } \\
\text { plate) }\end{array}$ & $\begin{array}{l}\text { De Long Mtns. } \\
\text { A-3 } \\
68^{\circ} 09^{\prime} 42.6^{\prime \prime} / \\
163^{\circ} 11^{\prime} 49.2^{\prime \prime}\end{array}$ & $\begin{array}{l}\text { Gnathodus texanus } \text { Roundy: } \\
12 \mathrm{~Pa}, 4 \mathrm{~Pb}, 1 \mathrm{M}, 1 \mathrm{Sb} \text {, and } 2 \mathrm{Sc} \text { elements [pl. } 1 \text {, } \\
\text { fig. 7] } \\
1 \text { juvenile Pa element Gnathodus sp. indet. } \\
1 \text { Pa element Rhachistognathus prolixus } \\
\text { Baesemann and Lane? } \\
1 \text { unassigned M element } \\
99 \text { indet. bar, blade, and platform fragments } \\
\text { CAI=3 } \\
\text { [DDH Su } 16 \text { (Su deposit), 558-577 ft; 29279-PC] }\end{array}$ & $\begin{array}{l}\text { middle Late Mississippian } \\
\text { (early Chesterian). }\end{array}$ & $\begin{array}{l}\text { Gnathodid biofacies: } \\
\text { open marine, likely } \\
\text { slope depositional } \\
\text { setting. }\end{array}$ & $\begin{array}{l}\text { Sample } \sim 109 \mathrm{ft} \text { below conformable contact of } \\
\text { Siksikpuk Formation and Ikalukrok unit and } \sim 421 \mathrm{ft} \\
\text { above main mineralized sulfide zone at Su deposit. } \\
\text { Samples several calcareous turbidites from lower } \\
\text { part of } \sim 100 \text {-ft-thick interval of interbedded } \\
\text { turbidites and black shale. See table } 2 \text { for Kivalina } \\
\text { unit sample from this drill hole. } \\
7.3 \text { kg of rock was processed. } \\
\text { Sample collected by D. Moore, I.L. Tailleur, and L.E. } \\
\text { Young. }\end{array}$ \\
\hline $\begin{array}{l}125 \\
\text { EMA (Red Dog } \\
\text { plate) }\end{array}$ & $\begin{array}{l}\text { De Long Mtns. } \\
\text { A-2 } \\
68^{\circ} 11^{\prime} 20^{\prime \prime /} \\
163^{\circ} 06^{\prime} 20^{\prime \prime}\end{array}$ & $\begin{array}{l}\text { Barren. No conodonts or other mineralized fossil } \\
\text { materials were found. } \\
\text { [DDH } 922 \text { (slightly west of Wulik deposit), } \\
\text { composite conodont sample } 1,45.5-46.5 \text { and 51.6- } \\
52.6 \mathrm{ft}]\end{array}$ & & & $\begin{array}{l}\text { Sample } \sim 32 \mathrm{ft} \text { below top of carbonate-rich partial } \\
\text { section of Ikalukrok unit that is at least } 950 \mathrm{ft} \text { thick } \\
\text { and not obviously structurally thickened. } \\
\text { Light-gray to medium-light-gray (outer), medium- } \\
\text { dark-gray (inner), medium-grained carbonate that is } \\
\text { laminated to very thin bedded with } 0.1 \text { to } 2 \mathrm{~mm} \text { dark } \\
\text { shale partings; some graded beds } \leq 2 \mathrm{~cm} \text { thick of } \\
\text { coarser grained carbonate turbidite, medium-light- } \\
\text { gray to light-gray (outer) and medium-gray (inner). } \\
\text { Thin section data: } \\
45.5,53 \mathrm{ft} \text { : Calcareous radiolarite; some radiolarians } \\
\text { preserved within calcite concretions } 1 \text { to } 2 \mathrm{~mm} \text { in } \\
\text { diameter. } \\
3.92 \mathrm{~kg} \text { of rock was processed. }\end{array}$ \\
\hline
\end{tabular}


Table 1. Conodont samples from the Ikalukrok unit of the Kuna Formation - Continued.

[All faunas identified by A.G. Harris. Structural units determined by L.E. Young. EMA, Endicott Mountains allochthon; PCA, Picnic Creek allochthon. CAI, conodont color alteration index. Letters in field number refer to collector: AD, J.A. Dumoulin EK, I El theck; and KE, K. Evans. DDH, diamond drill hole. Interval thicknesses in drill holes are drilled thicknesses and have not been corrected for dip of bedding. All drill hole samples collected by J.A. Dumoulin and (or) A.G. Harris unless otherwise indicated. Lithologic data under remarks are field descriptions unless otherwise indicated; thin section observations by J.A. Dumoulin. No., number; indet., indeterminate; loc., locality]

\begin{tabular}{|c|c|c|c|c|c|}
\hline $\begin{array}{l}\text { Locality No., } \\
\text { structural unit }\end{array}$ & $\begin{array}{l}\text { Quadrangle, } \\
\text { latitude n./ } \\
\text { longitude w. }\end{array}$ & $\begin{array}{c}\text { Conodont fauna and CAI } \\
\text { [field No.; USGS collection No.] }\end{array}$ & Age & $\begin{array}{c}\text { Conodont biofacies and } \\
\text { depositional } \\
\text { environment }\end{array}$ & Remarks \\
\hline \multirow[t]{2}{*}{$\begin{array}{l}125 \\
\text { EMA (Red Dog } \\
\text { plate) } \\
\text { [cont.] }\end{array}$} & \multirow[t]{2}{*}{$\begin{array}{l}\text { De Long Mtns. } \\
\text { A-2 } \\
68^{\circ} 11^{\prime} 20^{\prime \prime} / \\
163^{\circ} 06^{\prime} 20^{\prime \prime}\end{array}$} & $\begin{array}{l}\text { Barren. No conodonts or other mineralized fossil } \\
\text { materials were found. } \\
\text { [DDH 922, composite conodont sample 2, 163- } \\
164.5 \mathrm{ft}]\end{array}$ & & & $\begin{array}{l}\text { Sample } \sim 150 \mathrm{ft} \text { below top of carbonate-rich partial } \\
\text { section of Ikalukrok unit. } \\
\text { Medium-light-gray (outer), medium-dark-gray (inner), } \\
\text { very fine to fine-grained carbonate turbidite with } \\
15 \% \text { calcite veins and }<2 \% \text { mudstone layers. Thin } \\
\text { section at } 164.5 \mathrm{ft} \text { is calcareous radiolarite. } \\
\text { Heavy-mineral concentrate includes minor pyritized } \\
\text { triaxon sponge spicules. } \\
1.7 \mathrm{~kg} \text { of rock was processed. }\end{array}$ \\
\hline & & $\begin{array}{l}\text { Barren. No conodonts or other mineralized fossil } \\
\text { materials were found. } \\
\text { [DDH 922, composite conodont sample 3, 530-550 } \\
\text { ft] }\end{array}$ & & & $\begin{array}{l}\text { Sample } \sim 517 \mathrm{ft} \text { below top of carbonate-rich partial } \\
\text { section of Ikalukrok unit } \\
\text { Very light gray to light-gray (outer), medium-light- } \\
\text { gray to light-gray (inner) limestone in } 2 \mathrm{~mm} \text { to } 2 \mathrm{~cm} \\
\text { layers with mud breaks }<1 \mathrm{~mm} \text { thick. } \\
\text { Thin section data: } \\
538.5 \text { and } 545.5 \mathrm{ft} \text { : Calcitized radiolarians and } \\
\text { carbonate concretions, } 1 \text { to } 2 \mathrm{~mm} \text { in diameter, that } \\
\text { contain one or more radiolarians (some pyritized), in } \\
\text { a matrix of brown, noncarbonate mud. } \\
540.5,545 \mathrm{ft} \text {. Calcareous radiolarite; } 540.5 \text { is poorly } \\
\text { preserved and cross-cut by calcite veins; } 545 \\
\text { contains lenses of noncarbonate mud. } \\
547 \text { : Brown, noncarbonate mud with angular quartz silt } \\
\text { and locally abundant calcitized radiolarians. } \\
8.56 \mathrm{~kg} \text { of rock was processed. }\end{array}$ \\
\hline
\end{tabular}


Table 1. Conodont samples from the Ikalukrok unit of the Kuna Formation - Continued.

[All faunas identified by A.G. Harris. Structural units determined by L.E. Young. EMA, Endicott Mountains allochthon; PCA, Picnic Creek allochthon. CAI, conodont color alteration index. Letters in field number refer to collector: AD, J.A. Dumoulin

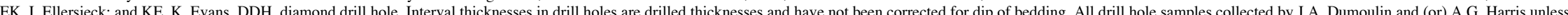
otherwise indicated. Lithologic data under remarks are field descriptions unless otherwise indicated; thin section observations by J.A. Dumoulin. No., number; indet., indeterminate; loc., locality]

\begin{tabular}{|c|c|c|c|c|c|}
\hline $\begin{array}{l}\text { Locality No., } \\
\text { structural unit }\end{array}$ & $\begin{array}{l}\text { Quadrangle, } \\
\text { latitude n./ } \\
\text { longitude w. }\end{array}$ & $\begin{array}{c}\text { Conodont fauna and CAI } \\
\text { [field No.; USGS collection No.] }\end{array}$ & Age & $\begin{array}{c}\text { Conodont biofacies and } \\
\text { depositional } \\
\text { environment }\end{array}$ & Remarks \\
\hline $\begin{array}{l}128 \\
\text { EMA (Red Dog } \\
\text { plate; } \\
\text { Competition } \\
\text { Creek subplate) }\end{array}$ & $\begin{array}{l}\text { De Long Mtns. } \\
\text { A-2 } \\
68^{\circ} 10^{\prime} 03^{\prime \prime} / \\
162^{\circ} 58^{\prime} 29^{\prime \prime}\end{array}$ & $\begin{array}{l}13 \text { indet. bar and blade fragments } \\
\text { CAI=3 } \\
\text { [DDH } 807 \text { (Anarraaq deposit), composite conodont } \\
\text { sample 1: 39-47.7 ft; 33491-PC] }\end{array}$ & $\begin{array}{l}\text { Unit known to be } \\
\text { Mississippian in age. }\end{array}$ & $\begin{array}{l}\text { Indeterminate (too few } \\
\text { conodonts). }\end{array}$ & $\begin{array}{l}\text { Sample } \sim 40 \mathrm{ft} \text { below top of highest of three structural } \\
\text { repeats of Ikalukrok unit (drill hole begins in } \\
\text { Ikalukrok). Sample is calcareous lithic turbidite } ~ 9 \mathrm{ft} \\
\text { thick, interbedded with siliceous mudstone and } \\
\text { chert. Subplate nomenclature from S. Jennings. } \\
39-47.7 \mathrm{ft} \text { : Light- to medium-light-gray (outer) and } \\
\text { medium-dark-gray (inner) calcareous sandstone or } \\
\text { sandy limestone containing very irregular lime mud } \\
\text { rip-up clasts as much as } 2 \mathrm{~cm} \text { long and some } \\
\text { burrows. No grading noted; could be more than one } \\
\text { event amalgamated and homogenized by burrowing. } \\
\text { Thin section data: } \\
40.8 \text { and } 42 \mathrm{ft} \text {. Lithic turbidites, with quartz, mica, } \\
\text { lithic clasts (mudstone, carbonate, chert, and } \\
\text { calcareous radiolarite), and productid spines (some } \\
\text { replaced by silica) in carbonate matrix. } \\
\text { Heavy-mineral concentrate includes rare tan to white } \\
\text { mica and phosphatized radiolarian steinkerns. } \\
\text { Light-mineral residue includes possible fluorite. } \\
4.2 \text { kg of rock was processed. }\end{array}$ \\
\hline $\begin{array}{l}\text { (Competition } \\
\text { Creek subplate) }\end{array}$ & & $\begin{array}{l}2 \text { indet. bar and blade fragments } \\
\text { CAI=3.5 } \\
\text { [DDH 807, composite conodont sample 2: 166.5- } \\
73.5 \text { and } 174-75 \mathrm{ft} ; 33492-\mathrm{PC} \text { ] }\end{array}$ & $\begin{array}{l}\text { Unit known to be } \\
\text { Mississippian in age. }\end{array}$ & $\begin{array}{l}\text { Indeterminate (too few } \\
\text { conodonts). }\end{array}$ & $\begin{array}{l}\text { Sample } \sim 167 \mathrm{ft} \text { below top of highest of three structural } \\
\text { repeats of Ikalukrok unit (drill hole begins in } \\
\text { Ikalukrok). Sample is calcareous lithic turbidite } \sim 10 \\
\mathrm{ft} \text { thick. } \\
166.5-175.0 \mathrm{ft} \text { : Carbonate turbidite, graded from } \\
\text { medium to fine sand and silt-size grains; calcareous } \\
\text { sandstone is medium dark gray (outer) and medium } \\
\text { gray and dark gray (inner). Locally calcareous black } \\
\text { mudstone lies above and below turbidite. } \\
\text { Thin section data: } \\
167.5,169,171.5 \mathrm{ft}: \text { Lithic turbidites, with clasts of } \\
\text { quartz, carbonate, chert, altered feldspar, and crinoid } \\
\text { ossicles in matrix of brown mud and local carbonate } \\
\text { cement. } \\
4.34 \mathrm{~kg} \text { of rock was processed. }\end{array}$ \\
\hline
\end{tabular}


Table 1. Conodont samples from the Ikalukrok unit of the Kuna Formation - Continued.

[All faunas identified by A.G. Harris. Structural units determined by L.E. Young. EMA, Endicott Mountains allochthon; PCA, Picnic Creek allochthon. CAI, conodont color alteration index. Letters in field number refer to collector: AD, J.A. Dumoulin EK, I. Ellerick; a otherwise indicated. Lithologic data under remarks are field descriptions unless otherwise indicated; thin section observations by J.A. Dumoulin. No., number; indet., indeterminate; loc., localityl

\begin{tabular}{|c|c|c|c|c|c|}
\hline $\begin{array}{l}\text { Locality No., } \\
\text { structural unit }\end{array}$ & $\begin{array}{l}\text { Quadrangle, } \\
\text { latitude n./ } \\
\text { longitude w. }\end{array}$ & $\begin{array}{c}\text { Conodont fauna and CAI } \\
\text { [field No.; USGS collection No.] }\end{array}$ & Age & \begin{tabular}{|c|}
$\begin{array}{c}\text { Conodont biofacies and } \\
\text { depositional } \\
\text { environment }\end{array}$ \\
\end{tabular} & Remarks \\
\hline \begin{tabular}{|l|}
128 \\
EMA (Red Dog \\
plate; Gul \\
subplate) \\
[cont.]
\end{tabular} & $\begin{array}{l}\text { De Long Mtns. } \\
\text { A-2 } \\
68^{\circ} 10^{\prime} 03^{\prime \prime} / \\
162^{\circ} 58^{\prime} 29^{\prime \prime}\end{array}$ & 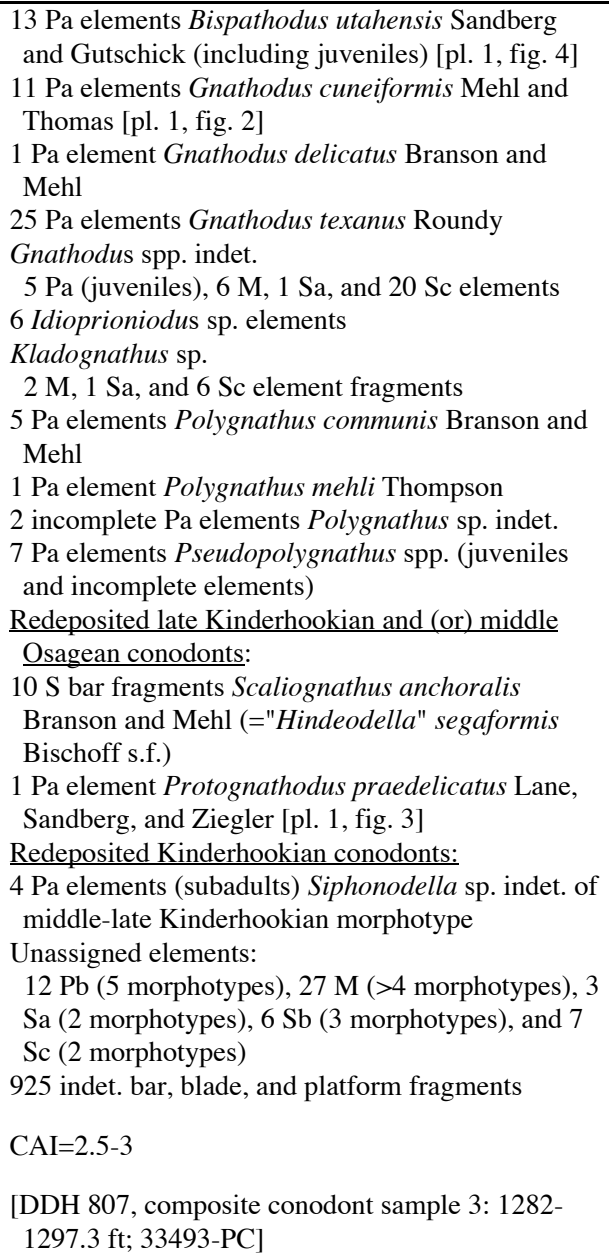 & $\begin{array}{l}\text { No older than late late Early } \\
\text { Mississippian (no older than } \\
\text { late Osagean, Po. mehli- } \\
\text { Lower G. texanus Zone); } \\
\text { contains redeposited older } \\
\text { Early Mississippian } \\
\text { (Kinderhookian and middle } \\
\text { Osagean) conodonts. }\end{array}$ & $\begin{array}{l}\text { Mixed biofacies; } \\
\text { postmortem transport } \\
\text { within or from the } \\
\text { gnathodid biofacies } \\
\text { suggesting at least a } \\
\text { slope or deeper water } \\
\text { depositional setting. } \\
\text { Fauna includes } \\
\text { redeposited conodonts } \\
\text { of mixed Early } \\
\text { Mississippian ages. }\end{array}$ & $\begin{array}{l}\text { Sample } \sim 72 \mathrm{ft} \text { below top of second of three structural } \\
\text { repeats of Ikalukrok unit; this interval of Ikalukrok } \\
\text { is fault-bounded above and below. Sample from } \\
\text { lower part of } \sim 25 \text {-ft-thick interval of calcareous } \\
\text { lithic turbidites. } \\
1282-1297.3 \mathrm{ft} \text { : Light-gray to medium-light-gray } \\
\text { (outer) and medium-dark-gray (inner) massive sandy } \\
\text { limestone, with fining-upward cycles of medium to } \\
\text { fine sand-sized carbonate, locally cross laminated, in } \\
\text { sets } 0.5 \text { to } 2 \mathrm{~cm} \text { thick; stylolites separate many } \\
\text { graded sets. Calcite spar veins }<1 \% \text {. } \\
\text { Thin section data: } \\
1287 \mathrm{ft}: \text { Lithic turbidite with } 0.5 \text {-cm-thick light and } \\
\text { dark layers and abundant radiolarians. Contains } \\
\text { calcite cement and common carbonate clasts; other } \\
\text { clasts include quartz, chert, siltstone, and zircon(?). } \\
1294 \mathrm{ft}: \text { Lithic turbidite with uniform texture. Contains } \\
\text { calcite cement and common carbonate clasts; other } \\
\text { clasts include quartz, chlorite, crinoid ossicles, and } \\
\text { productid spines. } \\
1298.7 \mathrm{ft} \text { : Mostly noncarbonate clasts in a brown } \\
\text { noncarbonate mud matrix. Clasts include brown } \\
\text { mudstone to } 1.4 \text { mm in diameter, quartz, productid } \\
\text { spines, and rare crinoid ossicles. } \\
\text { Heavy-mineral concentrate contains phosphatized } \\
\text { grains (including radiolarians?), lesser pyritized } \\
\text { radiolarians and sponge spicules, phosphatized } \\
\text { sponge spicules, and lesser sphalerite(?). } \\
\text { Light-mineral residue includes spiculitic carbonate } \\
\text { rock fragments. } \\
6.0 \text { kg of rock was processed. }\end{array}$ \\
\hline
\end{tabular}


Table 1. Conodont samples from the Ikalukrok unit of the Kuna Formation - Continued.

[All faunas identified by A.G. Harris. Structural units determined by L.E. Young. EMA, Endicott Mountains allochthon; PCA, Picnic Creek allochthon. CAI, conodont color alteration index. Letters in field number refer to collector: AD, J.A. Dumoulin EK, I. Ellersieck; and KE, K. Evans. DDH, diamond drill hole. Interval thicknesses in drill holes are drilled thicknesses and have not been cobrect for dip of bedding. All drill hole samples collected by J.A. Dumoulin and (or) A.G. Harris unless otherwise indicated. Lithologic data under remarks are field descriptions unless otherwise indicated; thin section observations by J.A. Dumoulin. No., number; indet., indeterminate; loc., locality]

\begin{tabular}{|c|c|c|c|c|c|}
\hline $\begin{array}{l}\text { Locality No., } \\
\text { structural unit }\end{array}$ & $\begin{array}{l}\text { Quadrangle, } \\
\text { latitude n./ } \\
\text { longitude w. }\end{array}$ & $\begin{array}{c}\text { Conodont fauna and CAI } \\
\text { [field No.; USGS collection No.] }\end{array}$ & Age & $\begin{array}{c}\text { Conodont biofacies and } \\
\text { depositional } \\
\text { environment }\end{array}$ & Remarks \\
\hline $\begin{array}{l}128 \\
\text { EMA (Red Dog } \\
\text { plate; Gul } \\
\text { subplate) } \\
\text { [cont.] }\end{array}$ & $\begin{array}{l}\text { De Long Mtns. } \\
\text { A-2 } \\
68^{\circ} 10^{\prime} 03^{\prime \prime} / \\
162^{\circ} 58^{\prime} 29^{\prime \prime}\end{array}$ & $\begin{array}{l}26 \text { small indet. bar and blade fragments } \\
\text { CAI=3.5 } \\
\text { [DDH 807, composite conodont sample 4: 1357.5, } \\
\text { 1358.8-60, 1360.6-62.0, 1362.6-64, 1367.0-67.5, } \\
1368-69.5,1370.5-71.5 \mathrm{ft} ; 33494-\mathrm{PC}]\end{array}$ & $\begin{array}{l}\text { Unit known to be } \\
\text { Mississippian in age. }\end{array}$ & $\begin{array}{l}\text { Indeterminate (too few } \\
\text { conodonts). Distal } \\
\text { winnow. }\end{array}$ & $\begin{array}{l}\text { Sample } ~ 147 \mathrm{ft} \text { below top of second of three structural } \\
\text { repeats of Ikalukrok unit; this interval of Ikalukrok } \\
\text { is fault-bounded above and below. Sample from 18- } \\
\text { ft-thick calcareous lithic turbidite. } \\
1357.5-1371.5 \mathrm{ft} \text { : Single carbonate turbidite grading } \\
\text { upward from 1) poorly sorted, medium-grained, } \\
\text { noncalcareous sandstone with 10-15\% mudstone } \\
\text { flasers, rip-ups, and laminae, to 2) alternations of } \\
\text { massive calcareous sandstone (in layers } \leq 4 \mathrm{ft} \text { ) and } \\
\text { graded intervals (up to } 2.5 \mathrm{ft} \text { ) of calcareous } \\
\text { sandstone to sandy limestone, to } 3 \text { ) bioturbated } \\
\text { medium-dark- to medium-gray siltstone and very } \\
\text { fine grained sandstone, and, finally, to } 4 \text { ) brownish- } \\
\text { black (outer) and dark-gray (inner) mudstone about } \\
8 \text { cm thick. } \\
\text { Thin section data: } \\
1370.7 \mathrm{ft}: \text { Lithic turbidite with abundant carbonate } \\
\text { clasts and calcite cement; also crinoid ossicles, } \\
\text { productid spines, phosphatic bioclasts, and clasts of } \\
\text { quartz, brown mud, and chlorite. } \\
1374 \mathrm{ft} \text { : Finely interlayered calcareous silty mudstone } \\
\text { with small bioclasts and quartz sandstone with minor } \\
\text { productid spines and crinoid ossicles. } \\
6.0 \text { kg of rock was processed. }\end{array}$ \\
\hline (Gul subplate) & & $\begin{array}{l}\text { Barren } \\
\text { [DDH 807, composite conodont sample 5: 1459.6- } \\
68.0 \mathrm{ft}]\end{array}$ & & & $\begin{array}{l}\text { Sample } 249 \mathrm{ft} \text { below top of second of three structural } \\
\text { repeats of Ikalukrok unit; this interval of Ikalukrok } \\
\text { is fault-bounded above and below. Sample from 12- } \\
\text { ft-thick calcareous lithic turbidite. } \\
\text { 1459.6-68.0 ft: Poorly sorted, calcareous siltstone to } \\
\text { fine-grained sandstone with remnant sedimentary } \\
\text { layering partly obscured by burrowing. } \\
\text { Thin section data: } \\
1462.8 \mathrm{ft}: \text { Lithic turbidite with clasts of quartz, } \\
\text { carbonate, and mudstone in calcite cement; bioclasts } \\
\text { include foraminifers and pyritized spines. } \\
7.28 \mathrm{~kg} \text { of rock was processed. }\end{array}$ \\
\hline
\end{tabular}


Table 1. Conodont samples from the Ikalukrok unit of the Kuna Formation - Continued.

[All faunas identified by A.G. Harris. Structural units determined by L.E. Young. EMA, Endicott Mountains allochthon; PCA, Picnic Creek allochthon. CAI, conodont color alteration index. Letters in field number refer to collector: AD, J.A. Dumoulin EK, I. Ellersick; a KE, K. Evans. DDH, diamond drill hole. Interval thicknesses in drill holes are drilled thickeses and have not been conected for dip of bedding. All drill hole samples collected by J.A. Dumoulin and (or) A. G. Harris unles otherwise indicated. Lithologic data under remarks are field descriptions unless otherwise indicated; thin section observations by J.A. Dumoulin. No., number; indet., indeterminate; loc., locality]

\begin{tabular}{|c|c|c|c|c|c|}
\hline $\begin{array}{l}\text { Locality No., } \\
\text { structural unit }\end{array}$ & $\begin{array}{l}\text { Quadrangle, } \\
\text { latitude n./ } \\
\text { longitude w. }\end{array}$ & $\begin{array}{c}\text { Conodont fauna and CAI } \\
\text { [field No.; USGS collection No.] }\end{array}$ & Age & $\begin{array}{c}\text { Conodont biofacies and } \\
\text { depositional } \\
\text { environment }\end{array}$ & Remarks \\
\hline $\begin{array}{l}129 \\
\text { EMA (Red Dog } \\
\text { plate) }\end{array}$ & $\begin{array}{l}\text { De Long Mtns. } \\
\text { A-2 } \\
68^{\circ} 09^{\prime} 50^{\prime \prime /} \\
162^{\circ} 57^{\prime} 35^{\prime \prime}\end{array}$ & $\begin{array}{l}\text { Barren. No conodonts or other mineralized fossil } \\
\text { materials were found. } \\
\text { [DDH 924 (Anarraaq deposit), composite conodont } \\
\text { sample: } 2008.5-2009.5,2013-2014.5,2015.7- \\
\text { 2017, 2017.5-2019 ft] }\end{array}$ & & & $\begin{array}{l}\text { Sample } ~ 330 \mathrm{ft} \text { below gradational contact of Siksikpuk } \\
\text { Formation and Ikalukrok unit, } \sim 80 \mathrm{ft} \text { below base of } \\
\text { thick barite interval at top of Ikalukrok, and } \sim 137 \mathrm{ft} \\
\text { above top of mineralized (sulfide) zone. Sample is } \\
\text { calcareous lithic turbidite }>12 \mathrm{ft} \text { thick. } \\
\text { 2008.5-2019: Medium- to medium-dark-gray (outer), } \\
\text { medium-dark- to dark-gray (inner) sandy limestone; } \\
\text { grades upward from very coarse grained to coarse- } \\
\text { grained, with pelmatozoan ossicles and round to } \\
\text { irregular pyrite and mud clasts, to medium- and fine- } \\
\text { grained. } \\
\text { Thin section data: } \\
\text { 2006.5, 2019 ft: Lithic turbidite with noncarbonate } \\
\text { mud matrix; clasts include quartz, carbonate, and } \\
\text { chert. } \\
6.75 \text { kg of rock was processed. } \\
\text { Sample from lithic turbidite zone of middle interval of } \\
\text { Ikalukrok unit of Kelley, Dumoulin, and Jennings } \\
\text { (2004). }\end{array}$ \\
\hline $\begin{array}{l}130 \\
\text { EMA (Red Dog } \\
\text { plate) }\end{array}$ & $\begin{array}{l}\text { De Long Mtns. } \\
\text { A-2 } \\
68^{\circ} 09^{\prime} 50^{\prime \prime /} / \\
162^{\circ} 57^{\prime} 22^{\prime \prime}\end{array}$ & $\begin{array}{l}\text { Barren } \\
\text { [DDH } 808 \text { (Anarraaq deposit), composite conodont } \\
\text { sample: 1935-36, 1938-39.5, 1941-41.3, 1943, } \\
\text { 1949, 1957, 1961, 1963, 1964.5, 1967, 1969, 1972, } \\
\text { 1973.5, and 1976 ft] }\end{array}$ & & & $\begin{array}{l}\text { Sample } \sim 367.5 \mathrm{ft} \text { below faulted contact of Siksikpuk } \\
\text { Formation and Ikalukrok unit, } \sim 14 \mathrm{ft} \text { below base of } \\
\text { thick barite interval at top of Ikalukrok, and } \sim 247 \mathrm{ft} \\
\text { above mineralized sulfide zone. Sample from } \\
\text { interval } \sim 50 \mathrm{ft} \text { thick of calcareous radiolarite and } \\
\text { lesser noncarbonate mudstone. } \\
\text { 1935-1976 ft: Varying proportions of medium- to dark- } \\
\text { gray (inner), very fine grained limestone with } \\
\text { scattered black mudstone(?) grains, and grayish- } \\
\text { black to black, locally siliceous mudstone. } \\
\text { Thin section data: } \\
1938,1955,1967,1976.7 \mathrm{ft} \text {. Calcareous radiolarite } \\
\text { with local siliceous laminae and barite veins. } \\
6.0 \text { kg of rock was processed. } \\
\text { Sample from chert and calcareous radiolarite zone of } \\
\text { middle interval of Ikalukrok unit of Kelley, } \\
\text { Dumoulin, and Jennings (2004). }\end{array}$ \\
\hline
\end{tabular}


Table 1. Conodont samples from the Ikalukrok unit of the Kuna Formation - Continued.

[All faunas identified by A.G. Harris. Structural units determined by L.E. Young. EMA, Endicott Mountains allochthon; PCA, Picnic Creek allochthon. CAI, conodont color alteration index. Letters in field number refer to collector: AD, J.A. Dumoulin EK, I. Ellersieck; and KE, K. Evans. DDH, diamond drill hole. Interval thicknesses in drill holes are drilled thicknesses and have not been corrected for dip of bedding. All drill hole samples collected by J.A. Dumoulin and (or) A.G. Harris unless otherwise indicated. Lithologic data under remarks are field descriptions unless otherwise indicated; thin section observations by J.A. Dumoulin. No., number; indet., indeterminate; loc., locality]

\begin{tabular}{|c|c|c|c|c|c|}
\hline $\begin{array}{l}\text { Locality No., } \\
\text { structural unit }\end{array}$ & $\begin{array}{l}\text { Quadrangle, } \\
\text { latitude n./ } \\
\text { longitude w. }\end{array}$ & $\begin{array}{c}\text { Conodont fauna and CAI } \\
\text { [field No.; USGS collection No.] }\end{array}$ & Age & \begin{tabular}{|c|} 
Conodont biofacies and \\
depositional \\
environment
\end{tabular} & Remarks \\
\hline \multirow[t]{2}{*}{$\begin{array}{l}131 \\
\text { EMA (Red Dog } \\
\text { plate) }\end{array}$} & \multirow[t]{2}{*}{$\begin{array}{l}\text { De Long Mtns. } \\
\text { A-2 } \\
68^{\circ} 09^{\prime} 46^{\prime \prime} / \\
162^{\circ} 57^{\prime} 44^{\prime \prime}\end{array}$} & $\begin{array}{l}\text { Barren. No conodonts or other mineralized fossil } \\
\text { materials were found. } \\
\text { [DDH } 813 \text { (Anarraaq deposit), composite conodont } \\
\text { sample 1: 2002.2-03.2 and 2004.8-06.0 ft] }\end{array}$ & & & $\begin{array}{l}\text { Sample } \sim 239 \mathrm{ft} \text { below conformable contact of } \\
\text { Siksikpuk Formation and Ikalukrok unit, } \sim 36 \mathrm{ft} \\
\text { below base of } 203 \text {-ft-thick barite interval, and } \sim 236 \\
\mathrm{ft} \text { above mineralized sulfide zone. Sample from } \sim 4- \\
\mathrm{ft} \text { interval of calcareous radiolarite and lesser } \\
\text { noncarbonate mudstone and chert. See table } 14 \text { for } \\
\text { radiolarian sample from this drill hole. } \\
\text { 2002.2-2006 ft: Mottled, medium- to medium-light- } \\
\text { gray (outer) and medium-dark-gray (inner) micrite } \\
\text { and carbonaceous micrite with probable radiolarians. } \\
\text { Thin section data: } \\
\text { 2002.5 ft: Calcareous radiolarite. } \\
\text { Sample weight not recorded. } \\
\text { Sample from chert and calcareous radiolarite zone of } \\
\text { middle interval of Ikalukrok unit of Kelley, } \\
\text { Dumoulin, and Jennings (2004). } \\
\end{array}$ \\
\hline & & $\begin{array}{l}3 \text { indet. bar or blade fragments } \\
\text { CAI cannot be readily determined because } \\
\text { conodonts are covered and (or) coated with } \\
\text { argillaceous and organic matter. CAI }=4 \text { or less } \\
\text { [DDH } 813 \text {, composite conodont sample 2: 2097.1- } \\
2098 \mathrm{ft}]\end{array}$ & $\begin{array}{l}\text { Mississippian (on the basis of } \\
\text { local stratigraphy). }\end{array}$ & $\begin{array}{l}\text { Indeterminate (too few } \\
\text { conodonts). }\end{array}$ & $\begin{array}{l}\text { Sample } \sim 131 \mathrm{ft} \text { below base of } 203-\mathrm{ft}-\text { thick barite } \\
\text { interval and } \sim 145 \mathrm{ft} \text { above mineralized sulfide zone. } \\
\text { Sample from } \sim 11 \mathrm{ft} \text { zone of carbonate turbidites. } \\
\text { 2097.1-2098 ft: Calcareous lithic turbidite with salt- } \\
\text { and-pepper texture; medium-light- to medium-dark- } \\
\text { gray (outer), medium-dark- and dark-gray (inner), } \\
\text { massive-bedded to mottled, medium- to fine- } \\
\text { grained. Contains rounded black chert(?) grains, } \\
\text { radiolarians(?), carbonate mud, and pyrite. } \\
\text { Thin section data: } \\
2098 \mathrm{ft} \text { Poorly preserved calcareous clasts in a } \\
\text { calcareous matrix, with some phosphate clasts and } \\
\text { pyritized radiolarians. } \\
0.92 \text { kg of rock was processed. } \\
\text { Sample from lithic turbidite zone of middle interval of } \\
\text { Ikalukrok unit of Kelley, Dumoulin, and Jennings } \\
\text { (2004). }\end{array}$ \\
\hline
\end{tabular}


Table 1. Conodont samples from the Ikalukrok unit of the Kuna Formation - Continued.

[All faunas identified by A.G. Harris. Structural units determined by L.E. Young. EMA, Endicott Mountains allochthon; PCA, Picnic Creek allochthon. CAI, conodont color alteration index. Letters in field number refer to collector: AD, J.A. Dumoulin EK, I. Eltersieck; and KE, K. Evans. DDH, diamond drill hole. Interval thicknesses in drill holes are drilled thicknesses and have not been corected for dip of bedding. All drill hole samples collected by J.A. Dumoulin and (or) A.G. Harris unless otherwise indicated. Lithologic data under remarks are field descriptions unless otherwise indicated; thin section observations by J.A. Dumoulin. No., number; indet., indeterminate; loc., locality]

\begin{tabular}{|c|c|c|c|c|c|}
\hline $\begin{array}{l}\text { Locality No., } \\
\text { structural unit }\end{array}$ & $\begin{array}{l}\text { Quadrangle, } \\
\text { latitude n./ } \\
\text { longitude w. }\end{array}$ & $\begin{array}{c}\text { Conodont fauna and CAI } \\
\text { [field No.; USGS collection No.] }\end{array}$ & Age & $\begin{array}{c}\text { Conodont biofacies and } \\
\text { depositional } \\
\text { environment }\end{array}$ & Remarks \\
\hline \multirow[t]{2}{*}{$\begin{array}{l}132 \\
\text { EMA (Red Dog } \\
\text { plate) }\end{array}$} & \multirow[t]{2}{*}{$\begin{array}{l}\text { De Long Mtns. } \\
\text { A-2 } \\
68^{\circ} 09^{\prime} 46^{\prime \prime} / \\
162^{\circ} 57^{\prime} 22^{\prime \prime}\end{array}$} & $\begin{array}{l}2 \text { indet. bar fragments } \\
\text { CAI=3.5 } \\
\text { [DDH } 810 \text { (Anarraaq deposit), composite conodont } \\
\text { sample 1: } 1919-40 \mathrm{ft} ; 33495-\mathrm{PC}]\end{array}$ & $\begin{array}{l}\text { Mississippian (on the basis of } \\
\text { local stratigraphy). }\end{array}$ & $\begin{array}{l}\text { Indeterminate (too few } \\
\text { conodonts). }\end{array}$ & $\begin{array}{l}\text { Sample } 360 \mathrm{ft} \text { below conformable contact of } \\
\text { Siksikpuk Formation and Ikalukrok unit, from basal } \\
\text { part of } 367 \text {-ft-thick barite interval, } \sim 216 \mathrm{ft} \text { above } \\
\text { mineralized sulfide zone. Sample from zone of } \\
\text { laminated barite with interbeds of calcareous } \\
\text { radiolarite and noncarbonate mudstone. } \\
\text { 1919-1940 ft: Laminated, medium-dark-gray, fine- } \\
\text { grained calcitized radiolarite (layers as much as 1- } \\
1.5 \mathrm{~cm} \text { thick), slightly calcareous mudstone, and } \\
\text { mudstone with local (<5\%) calcite spar veins. } \\
\text { Thin section data: } \\
\text { 1919, } 1920,1925,1940 \mathrm{ft} \text { : Calcareous radiolarite with } \\
\text { minor mud clasts and barite veins. Local fine } \\
\text { preservation of radiolarian test structure; some } \\
\text { radiolarians still silica, others pyritized. } \\
\text { Heavy-mineral concentrate chiefly composite } \\
\text { carbonaceous phosphatized grains with minor } \\
\text { fluorite(?). } \\
5.68 \mathrm{~kg} \text { of rock was processed. } \\
\text { Sample from basal part of barite interval of Ikalukrok } \\
\text { unit of Kelley, Dumoulin, and Jennings (2004). } \\
\end{array}$ \\
\hline & & $\begin{array}{l}1 \text { indet. bar fragment } \\
\text { CAI= 3 } \\
\text { [DDH 810, composite conodont sample 2: 2028- } \\
29.1 \mathrm{ft} ; 33496-\mathrm{PC}]\end{array}$ & $\begin{array}{l}\text { Mississippian (on the basis of } \\
\text { local stratigraphy). }\end{array}$ & $\begin{array}{l}\text { Indeterminate (too few } \\
\text { conodonts). }\end{array}$ & $\begin{array}{l}\text { Sample } \sim 102 \mathrm{ft} \text { below base of thick barite interval at } \\
\text { top of Ikalukrok unit and } \sim 127 \mathrm{ft} \text { above mineralized } \\
\text { sulfide zone; from } \sim 1.5 \text {-ft-thick lithic turbidite. } \\
2028.6-2029.1 \mathrm{ft}: \text { Medium-gray (outer) and dark-gray } \\
\text { (inner) calcareous sandstone bed with rounded black } \\
\text { chert(?) grains. } \\
\text { Thin section data: } \\
2028 \mathrm{ft} \text { : Lithic turbidite with quartz, carbonate and } \\
\text { mud clasts, crinoid ossicles, and calcite cement. } \\
1.3 \mathrm{~kg} \text { of rock was processed. } \\
\text { Sample from lithic turbidite zone of middle interval of } \\
\text { Ikalukrok unit of Kelley, Dumoulin, and Jennings } \\
\text { (2004). }\end{array}$ \\
\hline
\end{tabular}


Table 1. Conodont samples from the Ikalukrok unit of the Kuna Formation-Continued.

[All faunas identified by A.G. Harris. Structural units determined by L.E. Young. EMA, Endicott Mountains allochthon; PCA, Picnic Creek allochthon. CAI, conodont color alteration index. Letters in field number refer to collector: AD, J.A. Dumoulin EK, I Eller. otherwise indicated. Lithologic data under remarks are field descriptions unless otherwise indicated; thin section observations by J.A. Dumoulin. No., number; indet., indeterminate; loc., locality]

\begin{tabular}{|c|c|c|c|c|c|}
\hline $\begin{array}{l}\text { Locality No., } \\
\text { structural unit }\end{array}$ & $\begin{array}{l}\text { Quadrangle, } \\
\text { latitude n./ } \\
\text { longitude w. }\end{array}$ & $\begin{array}{c}\text { Conodont fauna and CAI } \\
\text { [field No.; USGS collection No.] }\end{array}$ & Age & \begin{tabular}{|c|}
$\begin{array}{c}\text { Conodont biofacies and } \\
\text { depositional } \\
\text { environment }\end{array}$ \\
\end{tabular} & Remarks \\
\hline $\begin{array}{l}133 \\
\text { EMA (Red Dog } \\
\text { plate) }\end{array}$ & $\begin{array}{l}\text { De Long Mtns. } \\
\text { A-2 } \\
68^{\circ} 09^{\prime} 41.8^{\prime \prime} / \\
162^{\circ} 57^{\prime} 22^{\prime \prime}\end{array}$ & $\begin{array}{l}\text { Barren. No conodonts or other mineralized fossil } \\
\text { materials were found } \\
\text { [DDH } 806 \text { (Anarraaq deposit), composite conodont } \\
\text { sample: } 1878,1881-82.5,1916,1940,1942 \text {, and } \\
1944.5 \mathrm{ft}]\end{array}$ & & & $\begin{array}{l}\text { Sample } \sim 282 \mathrm{ft} \text { below gradational contact of Siksikpuk } \\
\text { Formation and Ikalukrok unit, from basal part of } \\
365 \text {-ft-thick barite interval, } \sim 154 \mathrm{ft} \text { above } \\
\text { mineralized sulfide zone. Sample from zone of } \\
\text { calcareous and siliceous radiolarite interbedded with } \\
\text { locally calcareous barite and noncarbonate } \\
\text { mudstone. } \\
\text { 1878-1944.5 ft: Medium-dark-gray (outer) and dark- } \\
\text { gray (inner), laminated, fine-grained limestone } \\
\text { (calcareous radiolarite) and dark-gray (outer) } \\
\text { noncarbonate, partly burrowed siliceous mudstone. } \\
\text { Thin section data: } \\
1879,1942 \mathrm{ft}: \text { Calcareous radiolarite. } \\
1818.5 \mathrm{ft}: \text { Siliceous radiolarite with layers of } \\
\text { noncarbonate mud and pelloids. } \\
\text { Heavy-mineral concentrate contains abundant } \\
\text { composite phosphatized rock fragments. } \\
6.34 \mathrm{~kg} \text { of rock was processed. } \\
\text { Sample from basal part of barite interval of Ikalukrok } \\
\text { unit of Kelley, Dumoulin, and Jennings (2004). }\end{array}$ \\
\hline $\begin{array}{l}134 \\
\text { EMA (Red Dog } \\
\text { plate) }\end{array}$ & \begin{tabular}{|l} 
De Long Mtns. \\
A-2 \\
$68^{\circ} 09^{\prime} 40^{\prime \prime} /$ \\
$162^{\circ} 55^{\prime} 36.8^{\prime \prime}$
\end{tabular} & $\begin{array}{l}\text { 1 Pa element fragment of an ozarkodinid (likely } \\
\text { Bispathodus utahensis Sandberg and Gutschick) } \\
29 \text { indet. bar, blade, and platform fragments } \\
\text { CAI=3.5? or } 4 \text { ? but maybe less; conodonts have } \\
\text { adventitious organic matter, making CAI } \\
\text { determination difficult. } \\
\text { [DDH } 925 \text { (Anarraaq deposit), composite conodont } \\
\text { sample: } 877.5-878.5,879.5-880,882.5-883.5 \text {, } \\
884.5-885.5,886-886.7 \text {, and } 887-887.5 \mathrm{ft} \text {; 33622- } \\
\text { PC] }\end{array}$ & $\begin{array}{l}\text { Mississippian; likely late } \\
\text { Kinderhookian-Osagean. }\end{array}$ & $\begin{array}{l}\text { Indeterminate; small } \\
\text { size of conodont } \\
\text { fragments indicates a } \\
\text { postmortem winnow. }\end{array}$ & $\begin{array}{l}\text { Sample } \sim 180 \mathrm{ft} \text { below conformable contact of } \\
\text { Siksikpuk Formation and Ikalukrok unit. Sample is } \\
\text { fining upward calcareous lithic turbidite } \sim 15 \mathrm{ft} \text { thick. } \\
877.5-887.5: \text { Medium-light-gray at base and dark gray } \\
\text { at top (outer), medium-dark-gray at base and dark- } \\
\text { gray to grayish-black at top (inner), with graded } \\
\text { laminae, fine-scale cross laminations, and angular to } \\
\text { subrounded mud and pyrite clasts } \leq 1 \text { mm long. } \\
\text { Thin section at } 886.5 \mathrm{ft} \text { is diverse lithic turbidite with } \\
\text { abundant carbonate clasts and calcite cement; } \\
\text { bioclasts include crinoid ossicles, brachiopod(?) } \\
\text { fragments, phosphatic bioclasts, and silicified } \\
\text { productid spines. Other grains include angular quartz } \\
\text { and mud clasts. } \\
5.34 \text { kg of rock was processed. }\end{array}$ \\
\hline
\end{tabular}


Table 1. Conodont samples from the Ikalukrok unit of the Kuna Formation-Continued.

[All faunas identified by A.G. Harris. Structural units determined by L.E. Young. EMA, Endicott Mountains allochthon; PCA, Picnic Creek allochthon. CAI, conodont color alteration index. Letters in field number refer to collector: AD, J.A. Dumoulin

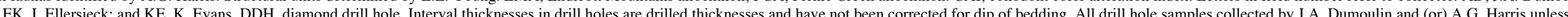
otherwise indicated. Lithologic data under remarks are field descriptions unless otherwise indicated; thin section observations by J.A. Dumoulin. No., number; indet., indeterminate; loc., locality]

\begin{tabular}{|c|c|c|c|c|c|}
\hline $\begin{array}{l}\text { Locality No., } \\
\text { structural unit }\end{array}$ & $\begin{array}{l}\text { Quadrangle, } \\
\text { latitude n./ } \\
\text { longitude w. }\end{array}$ & $\begin{array}{c}\text { Conodont fauna and CAI } \\
\text { [field No.; USGS collection No.] }\end{array}$ & Age & \begin{tabular}{|c|}
$\begin{array}{c}\text { Conodont biofacies and } \\
\text { depositional } \\
\text { environment }\end{array}$ \\
\end{tabular} & Remarks \\
\hline $\begin{array}{l}135 \\
\text { EMA (Red Dog } \\
\text { plate) }\end{array}$ & $\begin{array}{l}\text { De Long Mtns. } \\
\text { A-2 } \\
68^{\circ} 10^{\prime} 6.1^{\prime \prime} / \\
162^{\circ} 54^{\prime} 3.2^{\prime \prime}\end{array}$ & $\begin{array}{l}\text { All conodonts are completely or partly covered by } \\
\text { adventitious organic matter. } \\
1 \text { juvenile Pa element Bispathodus stabilis } \\
\text { (Branson and Mehl) or Bi. utahensis Sandberg and } \\
\text { Gutschick } \\
1 \text { unassigned incomplete Sb element } \\
15 \text { indet. bar and blade fragments } \\
\text { CAI=3 or } 4 \\
\text { [DDH } 780 \text { (east of Anarraaq deposit), } 760 \mathrm{ft} \text {; } \\
33483-\mathrm{PC}]\end{array}$ & $\begin{array}{l}\text { Early-middle Late } \\
\text { Mississippian } \\
\text { (Kinderhookian- } \\
\text { Meramecian). }\end{array}$ & $\begin{array}{l}\text { Indeterminate; too few } \\
\text { generically identifiable } \\
\text { conodonts. }\end{array}$ & $\begin{array}{l}\text { Samples upper } 1 \mathrm{ft} \text { of } 4 \text {-ft-thick carbonate turbidite in } \\
\text { upper part of Ikalukrok unit, } \sim 45-50 \text { feet below an } \\
\text { apparently unfaulted contact with the Siksikpuk } \\
\text { Formation and } 70 \text { feet above the mineralized sulfide } \\
\text { zone. } \\
\text { Medium- to dark-gray, finely laminated, very fine } \\
\text { grained limestone. Thin section is calcareous } \\
\text { radiolarite with abundant calcitized radiolarians. } \\
1.0 \text { kg of rock was processed. } \\
\text { Sample collected by D.L. Leach. }\end{array}$ \\
\hline $\begin{array}{l}139 \\
\text { EMA (Red Dog } \\
\text { plate) }\end{array}$ & $\begin{array}{l}\text { De Long Mtns. } \\
\text { A-2 } \\
68^{\circ} 05^{\prime} 0.1^{\prime \prime} / \\
162^{\circ} 49^{\prime} 23.4^{\prime \prime}\end{array}$ & $\begin{array}{l}\text { All conodonts have minor to extensive adventitious } \\
\text { carbonaceous matter and (or) pyrite making } \\
\text { taxonomic determination and CAI analysis } \\
\text { difficult. } \\
5 \text { P elements Bactrognathus sp. indet. } \\
3 \text { Hindeodella segaformis s.f. element fragments } \\
\text { (=S element bar fragments of Scaliognathus } \\
\text { anchoralis Branson and Mehl) } \\
3 \text { incomplete Pa elements Polygnathus communis } \\
\text { communis Branson and Mehl } \\
1 \text { Pa element fragment Polygnathus sp. indet. } \\
\text { Unassigned elements: } \\
5 \text { Pb (3 morphotypes), } 5 \mathrm{M} \text { ( } 2 \text { morphotypes), } 2 \mathrm{Sa} \text {, } \\
1 \mathrm{Sb} \text {, and } 6 \text { Sc (3 morphotypes) } \\
224 \text { indet. bar, blade, and platform fragments } \\
\text { CAI=2.5 or } 3 \\
\text { [DDH } 664 \text { (Paalaaq deposit), 1018.3-1020.5 ft; } \\
33621-\mathrm{PC}]\end{array}$ & $\begin{array}{l}\text { middle late Early } \\
\text { Mississippian (middle } \\
\text { Osagean; Sc. anchoralis-Do. } \\
\text { latus Zone) }\end{array}$ & $\begin{array}{l}\text { Postmortem transport } \\
\text { within or from a slope } \\
\text { or basin environment; } \\
\text { all conodonts are } \\
\text { incomplete and most } \\
\text { are relatively small } \\
\text { fragments suggesting, } \\
\text { at least in part, a } \\
\text { winnow. }\end{array}$ & $\begin{array}{l}\text { Sample near top of } \sim 65 \text {-ft-thick deformed, fault- } \\
\text { bounded interval of Ikalukrok unit. } \\
\text { 1018.3-1020.5: Medium-gray (outer), medium-dark- } \\
\text { gray (inner) calcareous radiolarite. } \\
\text { Thin section data: } \\
\text { 1018.5, } 1020.5: \text { calcareous radiolarite; relict } \\
\text { radiolarians preserved in concretions of radial } \\
\text { carbonate. } \\
3.3 \text { kg of rock was processed. }\end{array}$ \\
\hline $\begin{array}{l}144 \\
\text { EMA (Red Dog } \\
\text { plate) }\end{array}$ & $\begin{array}{l}\text { De Long Mtns. } \\
\text { A-2 } \\
68^{\circ} 04^{\prime} 37.6^{\prime \prime} / \\
162^{\circ} 49^{\prime} 8.7^{\prime \prime}\end{array}$ & $\begin{array}{l}\text { Barren } \\
\text { [DDH } 500 \text { (Aqqaluk deposit, } 360 \mathrm{ft}]\end{array}$ & & & $\begin{array}{l}\text { Sample from } \sim 20 \text {-ft-thick calcareous interval in a fault } \\
\text { bounded section of the Ikalukrok unit. } \\
\text { Very sooty, black, very fine grained limestone. Thin } \\
\text { section is calcareous radiolarite with }>30-40 \% \\
\text { radiolarians; some test structures well-preserved, but } \\
\text { most replaced by monocrystalline calcite. } \\
0.4 \text { kg of rock was processed. }\end{array}$ \\
\hline
\end{tabular}


Table 1. Conodont samples from the Ikalukrok unit of the Kuna Formation - Continued.

[All faunas identified by A.G. Harris. Structural units determined by L.E. Young. EMA, Endicott Mountains allochthon; PCA, Picnic Creek allochthon. CAI, conodont color alteration index. Letters in field number refer to collector: AD, J.A. Dumoulin EK, I. Ellersick; and KE, K. Evans. DDH, dimond drill hole. Interval thickneses in drill holes are drilled thicknesses and have not been corrected for dip of bedding. All drill hole samples collected by J.A. Dumoulin and (or) A.G. Harris unless otherwise indicated. Lithologic data under remarks are field descriptions unless otherwise indicated; thin section observations by J.A. Dumoulin. No., number; indet., indeterminate; loc., locality]

\begin{tabular}{|c|c|c|c|c|c|}
\hline $\begin{array}{l}\text { Locality No., } \\
\text { structural unit }\end{array}$ & $\begin{array}{l}\text { Quadrangle, } \\
\text { latitude n./ } \\
\text { longitude w. }\end{array}$ & $\begin{array}{c}\text { Conodont fauna and CAI } \\
\text { [field No.; USGS collection No.] }\end{array}$ & Age & \begin{tabular}{|c|} 
Conodont biofacies and \\
depositional \\
environment
\end{tabular} & Remarks \\
\hline $\begin{array}{l}149 \\
\text { EMA (Red Dog } \\
\text { plate) }\end{array}$ & $\begin{array}{l}\text { De Long Mtns. } \\
\text { A-2 } \\
68^{\circ} 04^{\prime} 26.5^{\prime \prime} / \\
162^{\circ} 50^{\prime} 06.7^{\prime \prime}\end{array}$ & $\begin{array}{l}\text { Unassigned elements: } \\
1 \mathrm{M} \text { and } 2 \mathrm{Sb} \\
7 \text { indet. bar fragments } \\
\mathrm{CAI}=4 \text { or less (CAI difficult to determine as all } \\
\text { conodonts are coated with organic matter). } \\
\text { [DDH } 205 \text { (Main deposit), } 239 \mathrm{ft} ; 33485-\mathrm{PC}]\end{array}$ & $\begin{array}{l}\text { Mississippian, as stratigraphic } \\
\text { identification is firm. }\end{array}$ & $\begin{array}{l}\text { Indeterminate (too few } \\
\text { conodonts). }\end{array}$ & $\begin{array}{l}\text { Sample } 10 \mathrm{ft} \text { below top of 14-ft interval of Ikalukrok } \\
\text { unit in fault contact below Kivalina unit. } \\
\text { One-ft-thick black limestone turbidite, interbedded } \\
\text { with black sooty mudstone. Thin section is } \\
\text { calcareous radiolarite; radiolarians } 80-90 \% \text { of thin } \\
\text { section. } \\
0.7 \mathrm{~kg} \text { of rock was processed. }\end{array}$ \\
\hline $\begin{array}{l}154 \\
\text { EMA (Red Dog } \\
\text { plate) }\end{array}$ & $\begin{array}{l}\text { De Long Mtns. } \\
\text { A-2 } \\
68^{\circ} 04^{\prime} 20.2^{\prime \prime} / \\
162^{\circ} 49^{\prime} 01.5^{\prime \prime}\end{array}$ & $\begin{array}{l}\text { Barren } \\
\text { [DDH } 8 \text { (Main Deposit), 95-100.5 ft] }\end{array}$ & & & $\begin{array}{l}\text { Chert and cherty shale with disseminated pyrite and } \\
\text { sphalerite, near top of zone of sulfide mineralization } \\
\text { in the Ikalukrok unit. See table } 2 \text { for Kivalina unit } \\
\text { sample from this drill hole. } \\
3 \mathrm{~kg} \text { of rock was processed in hydrofluoric acid. } \\
\text { Sample collected by D. Moore, I.L. Tailleur, and L.E. } \\
\text { Young. }\end{array}$ \\
\hline $\begin{array}{l}156 \\
\text { EMA (Red Dog } \\
\text { plate) }\end{array}$ & $\begin{array}{l}\text { De Long Mtns. } \\
\text { A-2 } \\
68^{\circ} 04^{\prime} 11^{\prime \prime} / \\
162^{\circ} 49^{\prime} 51^{\prime \prime}\end{array}$ & $\begin{array}{l}1 \text { Pa? element blade } \\
1 \text { indet. bar fragment } \\
\text { CAI= 3.5 } \\
\text { [DDH } 186 \text { (Main Deposit), } 94 \mathrm{ft} ; 33484-\mathrm{PC} \text { ) }\end{array}$ & $\begin{array}{l}\text { Mississippian, as stratigraphic } \\
\text { identification is firm. }\end{array}$ & $\begin{array}{l}\text { Indeterminate (too few } \\
\text { conodonts). }\end{array}$ & $\begin{array}{l}\text { Sample } 94 \mathrm{ft} \text { below top of } 125 \text {-ft-thick section of } \\
\text { Ikalukrok unit in fault contact above Kivalina unit. } \\
\text { Samples 1-ft-thick black limestone turbidite in 6-ft } \\
\text { interval of 2- to } 15 \text {-inch turbidites. Thin section is } \\
\text { calcareous radiolarite with very abundant } \\
\text { radiolarians and a slightly dolomitic matrix. } \\
0.62 \mathrm{~kg} \text { of rock was processed. }\end{array}$ \\
\hline $\begin{array}{l}157 \\
\text { EMA (Red Dog } \\
\text { plate) }\end{array}$ & $\begin{array}{l}\text { De Long Mtns. } \\
\text { A-2 } \\
68^{\circ} 03^{\prime} 24.3^{\prime \prime} / \\
162^{\circ} 49^{\prime} 40.6^{\prime \prime}\end{array}$ & $\begin{array}{l}\text { Barren } \\
\text { [DDH } 13 \text { (Qanaiyaq Deposit), 223-230 ft] }\end{array}$ & & & $\begin{array}{l}\text { Very pyritic bleached chert and shale from top of } \\
\text { mineralized interval in the Ikalukrok unit. } \\
1.1 \text { kg of rock was processed. } \\
\text { Sample collected by D. Moore, I.L. Tailleur, and L.E. } \\
\text { Young. }\end{array}$ \\
\hline
\end{tabular}


Table 2. Conodont samples from the Kivalina unit of the Kuna Formation

[All faunas identified by A.G. Harris. Structural units determined by L.E. Young. EMA, Endicott Mountains allochthon. CAI, conodont color alteration index. Letters in field number refer to collector: AD, J.A. Dumoulin; and Cx, S.M. Curtis. DDH, diamond drill hole. Interval thicknesses in drill holes are drilled thicknesses and have not been corrected for dip of bedding. All drill hole samples collected by J.A. Dumoulin and (or) A.G. Harris unless otherwise indicated. Lithologic data under remarks are field descriptions unless otherwise indicated; thin section observations by J.A. Dumoulin. No., number; indet., indeterminate; loc., locality]

\begin{tabular}{|c|c|c|c|c|c|}
\hline $\begin{array}{l}\text { Locality No., } \\
\text { structural unit }\end{array}$ & $\begin{array}{l}\text { Quadrangle, } \\
\text { latitude n./ } \\
\text { longitude w. }\end{array}$ & $\begin{array}{c}\text { Conodont fauna and CAI } \\
\text { [field No.; USGS collection No.] }\end{array}$ & Age & $\begin{array}{c}\text { Conodont biofacies and } \\
\text { depositional } \\
\text { environment }\end{array}$ & Remarks \\
\hline $\begin{array}{l}1 \\
\text { EMA (Red Dog } \\
\text { plate) }\end{array}$ & $\begin{array}{l}\text { De Long Mtns. } \\
\text { B-4 } \\
68^{\circ} 15^{\prime} 16.8^{\prime \prime} / \\
164^{\circ} 02^{\prime} 33^{\prime \prime}\end{array}$ & $\begin{array}{l}\text { Barren } \\
\text { [99AD23K] }\end{array}$ & & & $\begin{array}{l}\text { Sample near top of Kivalina unit, just below chert-rich } \\
\text { Ikalukrok unit. } \\
\text { Brown-gray, light-brown-gray-weathering, very fetid, } \\
\text { fine-grained limy dolostone in irregular } 5 \text { to } 7 \mathrm{~cm}- \\
\text { thick beds; local burrows, parallel laminae, and chert } \\
\text { bands. Some interbeds and layers of calcareous and } \\
\text { siliceous spiculite. Thin section is organic-rich, fine- } \\
\text { crystalline dolostone. } \\
\text { Heavy-mineral concentrate includes minor phosphatic } \\
\text { brachiopod fragments. } \\
\text { Weight of rock processed not recorded; likely 8-9 kg. }\end{array}$ \\
\hline \multirow[t]{2}{*}{$\begin{array}{l}17 \\
\text { EMA (Red Dog } \\
\text { plate) }\end{array}$} & \multirow[t]{2}{*}{$\begin{array}{l}\text { De Long Mtns. } \\
\text { A-3 } \\
68^{\circ} 07^{\prime} 48^{\prime \prime} / \\
163^{\circ} 33^{\prime} 00^{\prime \prime}\end{array}$} & $\begin{array}{l}\text { 10 Pa element fragments Bispathodus stabilis } \\
\text { (Branson and Mehl) or Bi. utahensis Sandberg and } \\
\text { Gutschick } \\
\text { Unassigned elements: } \\
1 \mathrm{M} \text { and } 1 \mathrm{Sa} \\
26 \text { indet. bar, blade, and platform fragments } \\
\text { CAI=3-4 } \\
\text { [79CX227 }+0 \mathrm{~m}]\end{array}$ & $\begin{array}{l}\text { latest Late Devonian-middle } \\
\text { Late Mississippian (late } \\
\text { Famennian-late } \\
\text { Meramecian). }\end{array}$ & $\begin{array}{l}\text { Postmortem transport } \\
\text { within or from a slope } \\
\text { or basin depositional } \\
\text { setting }\end{array}$ & $\begin{array}{l}\text { Submitted as Kivalina Limestone; mapped by Mayfield } \\
\text { and others (1990) as Kayak Shale (Mk1). Sample at } \\
\text { base of 47-m-thick section of carbonate and shale. } \\
\text { The conodonts are typical of the Kuna Formation but } \\
\text { are surface swimmers and occur in all environments. } \\
\text { Bi. stabilis and Bi. utahensis are most abundant in } \\
\text { deeper water deposits. Thus, their presence here } \\
\text { without other species strongly suggests a Kuna } \\
\text { depositional setting and not the Kayak Formation. } \\
\text { 3.3 kg of rock was processed. } \\
\text { Fossil loc. 33, Mayfield and others (1990). }\end{array}$ \\
\hline & & $\begin{array}{l}3 \text { indet. bar, blade, or platform fragments } \\
\text { CAI }=3-4 \\
{[79 \mathrm{CX} 227+20 \mathrm{~m}]}\end{array}$ & $\begin{array}{l}\text { Because sample is in the same } \\
\text { section and } 20 \mathrm{~m} \text { above } \\
79 \mathrm{CX} 227+0 \mathrm{~m} \text {, it is most } \\
\text { likely the same age. }\end{array}$ & $\begin{array}{l}\text { Indeterminate (too few } \\
\text { conodonts). }\end{array}$ & $\begin{array}{l}\text { Sample near mid-point of } 47-\mathrm{m} \text {-thick section of } \\
\text { carbonate and shale. } \\
2.72 \mathrm{~kg} \text { of rock was processed. }\end{array}$ \\
\hline $\begin{array}{l}30 \\
\text { EMA (Red Dog } \\
\text { plate) }\end{array}$ & $\begin{array}{l}\text { De Long Mtns. } \\
\text { A-3 } \\
68^{\circ} 04^{\prime} 01.8^{\prime \prime} / \\
163^{\circ} 12^{\prime} 30^{\prime \prime}\end{array}$ & $\begin{array}{l}1 \text { incomplete unassigned digyrate element } \\
2 \text { indet. bar and blade fragments } \\
\text { CAI }=\sim 4 \\
{[00 \mathrm{AD} 101 \mathrm{~A} ; 33640-\mathrm{PC}]}\end{array}$ & Mississippian & $\begin{array}{l}\text { Indeterminate (too few } \\
\text { conodonts). }\end{array}$ & $\begin{array}{l}\text { From type area of Kivalina unit of Kuna Formation. } \\
\text { Two-cm-thick nodular layers of orange-weathering, } \\
\text { medium- to dark-gray limestone containing sparse to } \\
\text { abundant crinoid ossicles and rare brachiopods. } \\
\text { Limestone beds separated by dark shale partings. } \\
\text { Thin section is skeletal wackestone with partly } \\
\text { dolomitized matrix; bioclasts include bryozoan, } \\
\text { pelmatozoan, and brachiopod fragments. } \\
\text { Heavy-mineral concentrate includes pyrite-bearing } \\
\text { silicified ostracodes (as single and complete valves) } \\
\text { and very scarce pyrite-bearing bryozoan fragments. } \\
12.2 \text { kg of rock was processed. }\end{array}$ \\
\hline
\end{tabular}


Table 2. Conodont samples from the Kivalina unit of the Kuna Formation-Continued.

[All faunas identified by A.G. Harris. Structural units determined by L.E. Young. EMA, Endicott Mountains allochthon. CAI, conodont color alteration index. Letters in field number refer to collector: AD, J.A. Dumoulin; and Cx, S.M. Curtis. DDH, diamond drill hole. Interval thicknesses in drill holes are drilled thicknesses and have not been corrected for dip of bedding. All drill hole samples collected by J.A. Dumoulin and (or) A.G. Harris unless otherwise indicated. Lithologic data under remarks are field descriptions unless otherwise indicated; thin section observations by J.A. Dumoulin. No., number; indet., indeterminate; loc., locality]

\begin{tabular}{|c|c|c|c|c|c|}
\hline $\begin{array}{l}\text { Locality No., } \\
\text { structural unit }\end{array}$ & $\begin{array}{l}\text { Quadrangle, } \\
\text { latitude n./ } \\
\text { longitude w. }\end{array}$ & $\begin{array}{c}\text { Conodont fauna and CAI } \\
\text { [field No.; USGS collection No.] }\end{array}$ & Age & $\begin{array}{c}\text { Conodont biofacies and } \\
\text { depositional } \\
\text { environment }\end{array}$ & Remarks \\
\hline $\begin{array}{l}1 \\
\text { EMA (Red Dog } \\
\text { plate) }\end{array}$ & $\begin{array}{l}\text { De Long Mtns. } \\
\text { A-2 } \\
68^{\circ} 08^{\prime} 18^{\prime \prime} / \\
162^{\circ} 48^{\prime} 36^{\prime \prime}\end{array}$ & $\begin{array}{l}3 \text { Pa element fragments Bispathodus stabilis } \\
\text { (Branson and Mehl) or Bi. utahensis Sandberg and } \\
\text { Gutschick } \\
1 \text { unassigned M element } \\
26 \text { indet. bar, blade, and platform fragments } \\
\text { CAI=3.5-4 } \\
\text { [8-16-83A; 29228-PC] }\end{array}$ & $\begin{array}{l}\text { late Late Devonian- early } \\
\text { Late Mississippian (late } \\
\text { Famennian-Meramecian). }\end{array}$ & $\begin{array}{l}\text { Indeterminate (too few } \\
\text { conodonts); normal- } \\
\text { marine depositional } \\
\text { setting. }\end{array}$ & $\begin{array}{l}\text { Kivalina unit, within } 100 \mathrm{ft} \text { of base of unit. } \\
\text { Chiefly black shale and scattered } 2 \text { to } 10 \mathrm{~cm} \text { beds of } \\
\text { very fine grained, silty, dark-gray limestone that } \\
\text { weathers light brown to grayish orange. } \\
8.2 \mathrm{~kg} \text { of rock was processed. } \\
\text { Sample collected by A.G. Harris. }\end{array}$ \\
\hline $\begin{array}{l}78 \\
\text { EMA (Red Dog } \\
\text { plate) }\end{array}$ & $\begin{array}{l}\text { De Long Mtns. } \\
\text { A-2 } \\
68^{\circ} 02^{\prime} 58^{\prime \prime} / \\
163^{\circ} 05^{\prime} 31^{\prime \prime}\end{array}$ & $\begin{array}{l}1 \text { incomplete juvenile Pa element Gnathodus sp. } \\
\text { indet. } \\
1 \text { juvenile Pa element Gnathodus texanus Roundy } \\
1 \text { Sb-Sc Kladognathus sp. indet. element } \\
1 \mathrm{M} \text { or S element fragment Synclydognathus sp. } \\
\text { indet. } \\
2 \text { juvenile P elements Vogelgnathus? sp. indet. } \\
19 \text { indet. bar, blade, and platform fragments } \\
\text { CAI=3.5 } \\
{[00 \mathrm{AD} 7 \mathrm{~A} ; 33628-\mathrm{PC}]}\end{array}$ & $\begin{array}{l}\text { late Early-middle Late } \\
\text { Mississippian (late } \\
\text { Osagean-early Chesterian). }\end{array}$ & $\begin{array}{l}\text { Indeterminate (too few } \\
\text { generically determinate } \\
\text { conodonts). Conodonts } \\
\text { indicate postmortem } \\
\text { winnow from a shelf } \\
\text { depositional setting. }\end{array}$ & $\begin{array}{l}\text { Sample from section of Kivalina unit that is } \\
\text { transitional, lithologically and faunally, to the } \\
\text { Utukok Formation. } \\
\text { Medium-light-gray- to orangish-light-gray-weathering, } \\
\text { dark- to medium-dark-gray wackestone/packstone } \\
\text { with pelmatozoan ossicles and columnals as much as } \\
3 \mathrm{~cm} \text { in diameter and large coral fragments as much } \\
\text { as } 40 \mathrm{~cm} \text { in size; bedding irregular, } 5 \text { to } 25 \mathrm{~cm} \text { thick, } \\
\text { with some minor bioturbation. } \\
\text { Thin section is bioturbated crinoidal packstone with } \\
\text { bryozoan and algal fragments; matrix partly } \\
\text { dolomitized. } \\
\text { Heavy-mineral concentrate contains fluorite and rare } \\
\text { ichthyoliths. } \\
\text { 10.46 kg of rock was processed. } \\
\text { Loc. } 18 \text { (fig. 2) of Dumoulin and others (2004). } \\
\text { Loc. } 42 \text { (Ellersieck and others, 1990) near here } \\
\text { contains foraminifers of late Early-Late } \\
\text { Mississippian (Zone } 11 \text { or younger) age. }\end{array}$ \\
\hline
\end{tabular}


Table 2. Conodont samples from the Kivalina unit of the Kuna Formation-Continued.

[All faunas identified by A.G. Harris. Structural units determined by L.E. Young. EMA, Endicott Mountains allochthon. CAI, conodont color alteration index. Letters in field number refer to collector: AD, J.A. Dumoulin; and Cx, S.M. Curtis. DDH, diamond drill hole. Interval thicknesses in drill holes are drilled thicknesses and have not been corrected for dip of bedding. All drill hole samples collected by J.A. Dumoulin and (or) A.G. Harris unless otherwise indicated. Lithologic data under remarks are field descriptions unless otherwise indicated; thin section observations by J.A. Dumoulin. No., number; indet., indeterminate; loc., locality]

\begin{tabular}{|c|c|c|c|c|c|}
\hline $\begin{array}{l}\text { Locality No., } \\
\text { structural unit }\end{array}$ & $\begin{array}{l}\text { Quadrangle, } \\
\text { latitude n./ } \\
\text { longitude w. }\end{array}$ & $\begin{array}{c}\text { Conodont fauna and CAI } \\
\text { [field No.; USGS collection No.] }\end{array}$ & Age & $\begin{array}{l}\text { Conodont biofacies and } \\
\text { depositional } \\
\text { environment }\end{array}$ & Remarks \\
\hline $\begin{array}{l}122 \\
\text { EMA (Red Dog } \\
\text { plate) }\end{array}$ & $\begin{array}{l}\text { De Long Mtns. } \\
\text { A-3 } \\
68^{\circ} 10^{\prime} 00^{\prime \prime} / \\
163^{\circ} 12^{\prime} 22^{\prime \prime}\end{array}$ & $\begin{array}{l}\text { Bispathodus stabilis (Branson and Mehl) or Bi. } \\
\text { utahensis Sandberg and Gutschick } \\
10 \mathrm{~Pa} \text { (juveniles and large adults), } 1 \mathrm{M} \text {, and } 1 \mathrm{Sa} \\
\text { elements } \\
1 \text { Idioprioniodus } \text { sp. indet. element fragment } \\
48 \text { indet. bar and blade fragments } \\
\text { CAI=3 } \\
\text { [DDH Su } 41 \text { (Su deposit), composite conodont } \\
\text { sample: } 1007.0-08.5,1018.0-21.5,1022.7-23.5 \text {, } \\
\text { and } 1024.0-24.4 \mathrm{ft} ; 33486-\mathrm{PC} \text { ] }\end{array}$ & $\begin{array}{l}\text { Early-early Late } \\
\text { Mississippian (middle } \\
\text { Kinderhookian- } \\
\text { Meramecian). }\end{array}$ & $\begin{array}{l}\text { Indeterminate (too few } \\
\text { conodonts); } \\
\text { postmortem transport } \\
\text { within or from the } \\
\text { bispathodid biofacies. } \\
\text { Conodonts suggest a } \\
\text { deep-water, off shelf or } \\
\text { off platform } \\
\text { depositional setting. }\end{array}$ & $\begin{array}{l}\text { Uppermost Kivalina unit, immediately below } \\
\text { gradational contact with overlying Ikalukrok unit. } \\
\text { 1007.0-1024. } 4 \mathrm{ft} \text { : Various proportions of laminated to } \\
\text { thin beds (up to } 3 \mathrm{~cm} \text { thick) of medium-light-gray } \\
\text { and medium- to dark-gray (outer) and medium-gray } \\
\text { to grayish-black (inner), chiefly very fine grained } \\
\text { limestone, calcareous mudstone, and noncalcareous } \\
\text { mudstone with <2 to 5\% calcite spar veins. Lower } \\
10 \mathrm{~cm} \text { of sampled interval grades upward from } \\
\text { coarse to very fine carbonate sand, silt(?), and, } \\
\text { finally, to micrite. Coarser intervals are skeletal } \\
\text { packstone-grainstone with dark-gray flattened mud } \\
\text { clasts several millimeters long; bioclasts mainly } \\
\text { pelmatozoan debris. } \\
\text { Thin section data: } \\
1008 \mathrm{ft} \text { : Fine-grained calcareous spiculite. } \\
1008.6,1016.3 \text {, and } 1020.8 \mathrm{ft} \text { : Finely interlayered } \\
\text { calcareous spiculite and skeletal packstone. } \\
1030 \mathrm{ft} \text { : Packed crinoidal packstone, partly silicified } \\
\text { and dolomitized, with crinoid ossicles to } 2 \text { mm. } \\
\text { Heavy-mineral concentrate contains phosphatic } \\
\text { brachiopod fragments and phosphatized brachiopod } \\
\text { spines. } \\
7.68 \text { kg of rock was processed. }\end{array}$ \\
\hline $\begin{array}{l}123 \\
\text { EMA (Red Dog } \\
\text { plate) }\end{array}$ & $\begin{array}{l}\text { De Long Mtns. } \\
\text { A-3 } \\
68^{\circ} 09^{\prime} 45.4^{\prime \prime} / \\
163^{\circ} 12^{\prime} 30.7^{\prime \prime}\end{array}$ & $\begin{array}{l}\text { Barren } \\
\text { [DDH Su } 31 \text { (Su deposit), 1806-1824ft] }\end{array}$ & & & $\begin{array}{l}\text { Uppermost Kivalina unit from just below mineralized } \\
\text { zone at base of Ikalukrok unit at Su deposit. Sample } \\
\text { from } 22 \text {-ft-thick interval of thinly interbedded black } \\
\text { limestone and dark gray to black shale. See table } 1 \\
\text { for Ikalukrok sample from this drill hole. } \\
7.8 \mathrm{~kg} \text { of rock was processed. } \\
\text { Sample collected by D. Moore, I.L. Tailleur, and L.E. } \\
\text { Young. }\end{array}$ \\
\hline $\begin{array}{l}124 \\
\text { EMA (Red Dog } \\
\text { plate) }\end{array}$ & $\begin{array}{l}\text { De Long Mtns. } \\
\text { A-3 } \\
68^{\circ} 09^{\prime} 42.6^{\prime \prime} / \\
163^{\circ} 11^{\prime} 49.2^{\prime \prime}\end{array}$ & $\begin{array}{l}\text { Barren } \\
\text { [DDH Su } 16 \text { (Su deposit), 1195-1216ft] }\end{array}$ & & & $\begin{array}{l}\text { Uppermost Kivalina unit, } 48 \mathrm{ft} \text { below mineralized } \\
\text { zone at base of Ikalukrok unit at Su deposit. Sample } \\
\text { from } 20 \text {-ft-thick interval of medium gray to black, } \\
\text { mostly limy shale, with pyrite and calcite veins. See } \\
\text { table } 1 \text { for Ikalukrok sample from this drill hole. } \\
7.46 \mathrm{~kg} \text { of rock was processed. } \\
\text { Sample collected by D. Moore, I.L. Tailleur, and L.E. } \\
\text { Young. }\end{array}$ \\
\hline
\end{tabular}


Table 2. Conodont samples from the Kivalina unit of the Kuna Formation-Continued.

[All faunas identified by A.G. Harris. Structural units determined by L.E. Young. EMA, Endicott Mountains allochthon. CAI, conodont color alteration index. Letters in field number refer to collector: AD, J.A. Dumoulin; and Cx, S.M. Curtis. DDH, diamond drill hole. Interval thicknesses in drill holes are drilled thicknesses and have not been corrected for dip of bedding. All drill hole samples collected by J.A. Dumoulin and (or) A.G. Harris unless otherwise indicated. Lithologic data under remarks are field descriptions unless otherwise indicated; thin section observations by J.A. Dumoulin. No., number; indet., indeterminate; loc., locality]

\begin{tabular}{|c|c|c|c|c|c|}
\hline $\begin{array}{l}\text { Locality No., } \\
\text { structural unit }\end{array}$ & $\begin{array}{l}\text { Quadrangle, } \\
\text { latitude } \mathrm{n} . / \\
\text { longitude w. }\end{array}$ & $\begin{array}{c}\text { Conodont fauna and CAI } \\
\text { [field No.; USGS collection No.] }\end{array}$ & Age & $\begin{array}{c}\text { Conodont biofacies and } \\
\text { depositional } \\
\text { environment }\end{array}$ & Remarks \\
\hline \multirow[t]{2}{*}{$\begin{array}{l}137 \\
\text { EMA (Red Dog } \\
\text { plate) }\end{array}$} & \multirow[t]{2}{*}{$\begin{array}{l}\text { DeLong Mtns. } \\
\text { A-2 } \\
68^{\circ} 05^{\prime} 22.8^{\prime \prime} / \\
162^{\circ} 50^{\prime} 29.4^{\prime \prime}\end{array}$} & $\begin{array}{l}\text { Barren. No conodonts or other mineralized fossil } \\
\text { materials were found. } \\
\text { [DDH } 777 \text { (north of Paalaaq deposit), composite } \\
\text { conodont sample 1: } 478-80.4 \text { and } 487.6-488 \mathrm{ft}]\end{array}$ & & & $\begin{array}{l}\text { Sample from upper part of } ~ 2500 \mathrm{ft} \text { thick interval of } \\
\text { Kivalina unit that is probably structurally thickened. } \\
478.0-488.0 \mathrm{ft} \text { : Light-brownish-gray and medium- } \\
\text { light-gray (outer), dark-gray (inner), fine-grained, } \\
\text { locally graded (in intervals } 0.5-4 \mathrm{~cm} \text { thick) } \\
\text { limestone and noncalcareous to weakly calcareous } \\
\text { mudstone and shale containing } ~ 5 \% \text { calcite spar } \\
\text { veins. } \\
\text { Thin section data: } \\
483.3 \mathrm{ft}: \text { Graded carbonate layers (to } 1 \mathrm{~cm} \text { thick), with } \\
\text { bioclasts (crinoids, spicules), peloids, and mud clasts } \\
\text { (to } 1.5 \mathrm{~mm} \text { ); thin interlayers of noncarbonate mud. } \\
486.9 \mathrm{ft} \text { : Fine-grained peloidal carbonate with minor } \\
\text { euhedral dolomite and quartz silt. } \\
489 \mathrm{ft} \text { : Dolomitic, calcareous spiculite with mud } \\
\text { intraclasts to } 1.5 \mathrm{~mm} \text { long. } \\
4.1 \mathrm{~kg} \text { of rock was processed. }\end{array}$ \\
\hline & & $\begin{array}{l}\text { Barren. No conodonts or other mineralized fossil } \\
\text { materials were found. } \\
\text { [DDH 777, composite conodont sample 2: 1088- } \\
89.5,1090-91,1096-97,1101-01.8,1104,1106- \\
07,1110.5-11.0 \text { and } 1117 \mathrm{ft} \text { ] }\end{array}$ & & & $\begin{array}{l}\text { Sample from middle part of } \sim 2500 \mathrm{ft} \text { thick interval of } \\
\text { Kivalina unit that is probably structurally thickened. } \\
1088.0-1117.0 \mathrm{ft} \text { : Interval is at least } 50 \% \text { carbonate. } \\
\text { Various proportions of medium-light- to medium- } \\
\text { gray (outer) and medium-dark-gray (inner) shaly } \\
\text { limestone to limestone and medium-dark- to dark- } \\
\text { gray (outer) and dark-gray to grayish-black (inner) } \\
\text { shale and calcareous shale. Coarsest layers are } \\
\text { medium- to coarse-grained supportstone with } \\
\text { pelmatozoan debris. Some layers bioturbated. } \\
\text { Thin section data: } \\
1088.5 \mathrm{ft}: \text { Interlayered noncarbonate mud, calcareous } \\
\text { spiculite, and crinoid grainstone with mud clasts and } \\
\text { quartz silt. } \\
1096.5 \mathrm{ft} \text { : Calcareous spiculite. } \\
1110 \mathrm{ft}: \text { Fine-grained bioturbated dolostone with minor } \\
\text { quartz silt. } \\
1111.3 \mathrm{ft} \text { : Cherty dolostone. } \\
\text { Heavy-mineral concentrate includes rare chalcopyrite. } \\
6.0 \mathrm{~kg} \text { of rock was processed. }\end{array}$ \\
\hline
\end{tabular}


Table 2. Conodont samples from the Kivalina unit of the Kuna Formation-Continued.

[All faunas identified by A.G. Harris. Structural units determined by L.E. Young. EMA, Endicott Mountains allochthon. CAI, conodont color alteration index. Letters in field number refer to collector: AD, J.A. Dumoulin; and Cx, S.M. Curtis. DDH, diamond drill hole. Interval thicknesses in drill holes are drilled thicknesses and have not been corrected for dip of bedding. All drill hole samples collected by J.A. Dumoulin and (or) A.G. Harris unless otherwise indicated. Lithologic data under remarks are field descriptions unless otherwise indicated; thin section observations by J.A. Dumoulin. No., number; indet., indeterminate; loc., locality]

\begin{tabular}{|c|c|c|c|c|c|}
\hline $\begin{array}{l}\text { Locality No., } \\
\text { structural unit }\end{array}$ & $\begin{array}{l}\text { Quadrangle, } \\
\text { latitude n./ } \\
\text { longitude w. }\end{array}$ & $\begin{array}{c}\text { Conodont fauna and CAI } \\
\text { [field No.; USGS collection No.] }\end{array}$ & Age & $\begin{array}{c}\text { Conodont biofacies and } \\
\text { depositional } \\
\text { environment } \\
\end{array}$ & Remarks \\
\hline \multirow[t]{3}{*}{$\begin{array}{l}137 \\
\text { EMA (Red Dog } \\
\text { plate) } \\
\text { [cont.] }\end{array}$} & \multirow[t]{3}{*}{$\begin{array}{l}\text { De Long Mtns. } \\
\text { A-2 } \\
68^{\circ} 05^{\prime} 22.8^{\prime \prime} / \\
162^{\circ} 50^{\prime} 29.4^{\prime \prime}\end{array}$} & $\begin{array}{l}\text { Barren. No conodonts or other mineralized fossil } \\
\text { materials were found. } \\
\text { [DDH 777, composite conodont sample } 3: 1370.5- \\
71.5,1372.5-75,1378-78.4,1382-82.2,1385.5- \\
86.5,1387.4-87.5 \text {, and } 1391-92 \mathrm{ft} \text { ] }\end{array}$ & & & $\begin{array}{l}\text { Sample from middle part of } ~ 2500 \mathrm{ft} \text { thick interval of } \\
\text { Kivalina unit that is probably structurally thickened. } \\
1370.5-1392.0 \mathrm{ft} \text { : Various proportions of medium- } \\
\text { dark- to dark-gray (outer), grayish-black (inner) } \\
\text { shale to slightly calcareous shale and very light- to } \\
\text { medium-gray (outer), medium-dark-gray (inner) } \\
\text { limestone (carbonate turbidite beds as much as } 4.5 \mathrm{ft} \\
\text { thick, graded and partly bioturbated). Thin section at } \\
1387.4 \mathrm{ft} \text { is fine-grained, euhedral to subhedral } \\
\text { dolomite(?) mosaic with sparse bioclasts (crinoids, } \\
\text { spicules). } \\
8.68 \mathrm{~kg} \text { of rock was processed. }\end{array}$ \\
\hline & & $\begin{array}{l}\text { 1 Pa element fragment Bispathodus utahensis } \\
\text { Sandberg and Gutschick } \\
1 \text { Pa element Polygnathus communis communis } \\
\text { Branson and Mehl } \\
1 \text { S element bar fragment Scaliognathus anchoralis } \\
\text { Branson and Mehl (="Hindeodella" segaformis } \\
\text { Bischoff s.f.) } \\
1 \text { Pb element of late Early Devonian-Early } \\
\text { Mississippian morphotype } \\
2 \text { Sc (2 morphotypes) elements of late Late } \\
\text { Devonian and Mississippian morphotype } \\
60 \text { indet. bar, blade, and platform fragments } \\
\text { CAI=3 } \\
\text { [DDH 777, composite conodont sample 4: 1961.2- } \\
62.0,1972.7-73.0,1974,1979,1980,1982,1989 \\
\text { ft; 33490-PC] }\end{array}$ & $\begin{array}{l}\text { middle late Early } \\
\text { Mississippian (middle } \\
\text { Osagean; Sc. anchoralis- } \\
\text { Do. latus Zone). }\end{array}$ & $\begin{array}{l}\text { Indeterminate (no } \\
\text { generically determinate } \\
\text { conodonts); forms } \\
\text { present indicate post- } \\
\text { mortem hydraulic } \\
\text { transport. }\end{array}$ & $\begin{array}{l}\text { Sample from lower part of } 2500 \mathrm{ft} \text { thick interval of } \\
\text { Kivalina unit that is probably structurally thickened. } \\
1961.2-1989.0 \mathrm{ft} \text { : Various proportions of medium- } \\
\text { dark-gray (outer), grayish-black (inner) shale to } \\
\text { calcareous shale, medium-dark-gray (inner) } \\
\text { laminated shaly limestone, and fine-grained } \\
\text { limestone with rare } 1 \text { to } 2 \mathrm{~mm} \text { coarser grained } \\
\text { laminae. A coarse-grained intraclast conglomerate } \\
\text { containing dark shale clasts up to } 7 \mathrm{~mm} \text { long is at } \\
1980 \mathrm{ft} \text {. Thin section at } 1979.9 \mathrm{ft} \text { is fine-grained } \\
\text { dolomite with } 3 \text {-cm-thick layer of coarse skeletal- } \\
\text { lithic clast supportstone. Bioclasts include crinoid } \\
\text { and bryozoan fragments. Other clasts (to } 4 \mathrm{~mm} \text { ) are } \\
\text { shale, micrite, and peloidal packstone. } \\
\text { Heavy-mineral concentrate includes scarce phosphatic } \\
\text { brachiopod fragments. } \\
7.25 \mathrm{~kg} \text { of rock was processed. }\end{array}$ \\
\hline & & $\begin{array}{l}\text { Barren. No conodonts or other mineralized fossil } \\
\text { materials were found. } \\
\text { [DDH 777, composite conodont sample 5: 2430.3- } \\
39.0 \mathrm{ft}]\end{array}$ & & & $\begin{array}{l}\text { Sample from lower part of } \sim 2500 \mathrm{ft} \text { thick interval of } \\
\text { Kivalina unit that is probably structurally thickened. } \\
2430.3-2439.0 \mathrm{ft} \text { : Medium-dark- to dark-gray (outer), } \\
\text { dark-gray to grayish black (inner) shaly limestone } \\
\text { and medium-light- to light-gray (outer) limestone. } \\
\text { Coarser grained carbonate intervals up to } 15 \mathrm{~cm} \\
\text { thick are fine to medium grained with crinoid debris. } \\
\text { Thin section data: } \\
2437 \mathrm{ft} \text {. Most of slide is fine laminae of noncarbonate } \\
\text { and carbonate mud with minor dolomite euhedra and } \\
\text { quartz silt; one layer of bioclasts (crinoid ossicles) } \\
\text { and clasts (micrite with calcareous spicules and } \\
\text { peloids) to } 1 \text { mm. Slide looks deformed. } \\
6.02 \mathrm{~kg} \text { of rock was processed. }\end{array}$ \\
\hline
\end{tabular}


Table 2. Conodont samples from the Kivalina unit of the Kuna Formation-Continued.

[All faunas identified by A.G. Harris. Structural units determined by L.E. Young. EMA, Endicott Mountains allochthon. CAI, conodont color alteration index. Letters in field number refer to collector: AD, J.A. Dumoulin; and Cx, S.M. Curtis. DDH, diamond drill hole. Interval thicknesses in drill holes are drilled thicknesses and have not been corrected for dip of bedding. All drill hole samples collected by J.A. Dumoulin and (or) A.G. Harris unless otherwise indicated. Lithologic data under remarks are field descriptions unless otherwise indicated; thin section observations by J.A. Dumoulin. No., number; indet., indeterminate; loc., locality]

\begin{tabular}{|c|c|c|c|c|c|}
\hline $\begin{array}{l}\text { Locality No., } \\
\text { structural unit }\end{array}$ & $\begin{array}{l}\text { Quadrangle, } \\
\text { latitude n./ } \\
\text { longitude w. }\end{array}$ & $\begin{array}{c}\text { Conodont fauna and CAI } \\
\text { [field No.; USGS collection No.] }\end{array}$ & Age & \begin{tabular}{|c|} 
Conodont biofacies and \\
depositional \\
environment
\end{tabular} & Remarks \\
\hline \multirow[t]{2}{*}{$\begin{array}{l}138 \\
\text { EMA (Red Dog } \\
\text { plate) }\end{array}$} & \multirow[t]{2}{*}{$\begin{array}{l}\text { De Long Mtns. } \\
\text { A-2 } \\
68^{\circ} 05^{\prime} 00.5^{\prime \prime} / \\
162^{\circ} 49^{\prime} 44.4^{\prime \prime}\end{array}$} & $\begin{array}{l}\text { 2 S element fragments Scaliognathus anchoralis } \\
\text { Branson and Mehl (="Hindeodella" segaformis } \\
\text { Bischoff s.f.) } \\
5 \text { indet. bar, blade, and platform fragments } \\
\text { CAI=3 } \\
\text { [DDH } 676 \text { (Paalaaq deposit), composite conodont } \\
\text { sample 1: 671.5-672.4 ft; 33489-PC] }\end{array}$ & $\begin{array}{l}\text { middle late Early } \\
\text { Mississippian (middle } \\
\text { Osagean; Sc. anchoralis- } \\
\text { Do. latus Zone). }\end{array}$ & $\begin{array}{l}\text { Indeterminate (too few } \\
\text { generically identifiable } \\
\text { conodonts). Conodonts } \\
\text { present indicate a post- } \\
\text { mortem winnow. }\end{array}$ & $\begin{array}{l}\text { Sample from lower part of } \sim 725 \mathrm{ft} \text { thick, highly } \\
\text { deformed interval of Kivalina unit, } \sim 213 \mathrm{ft} \text { below } \\
\text { altered keratophyre. } \\
671.5-672.4 \mathrm{ft} \text { : Graded turbidite bed made of light- to } \\
\text { medium-light-gray (outer), medium- to medium- } \\
\text { dark-gray (inner) carbonate sand, silt, and mud. } \\
\text { Thin section data: } \\
671.5 \mathrm{ft} \text { : Fine-grained dolostone. } \\
672.4 \mathrm{ft} \text { : Bioclastic supportstone with bryozoan and } \\
\text { pelmatozoan fragments and brown to black mud } \\
\text { clasts. } \\
\text { Heavy-mineral concentrate contains scarce phosphatic } \\
\text { brachiopod fragments. } \\
1.53 \mathrm{~kg} \text { of rock was processed. }\end{array}$ \\
\hline & & $\begin{array}{l}\text { Barren. No conodonts or other mineralized fossil } \\
\text { materials were found. } \\
\text { [DDH 676, composite conodont sample 2: 689.5- } \\
696.1 \mathrm{ft} \text { ] }\end{array}$ & & & $\begin{array}{l}\text { 689.5-696.1 ft: Chiefly a reverse-graded massive bed } \\
\text { (possibly partly homogenized by burrowing) of } \\
\text { light- to medium-gray (outer), medium-dark-gray } \\
\text { (inner), coarse- to fine-grained carbonate with local } \\
\text { dark-gray to black mud clasts. } \\
\text { Thin section data: } \\
689.6 \mathrm{ft} \text { : Diverse bioclasts and clasts of brown mud } \\
\text { and chert? (to } 1 \mathrm{~cm} \text { ) in fine-grained carbonate } \\
\text { matrix. } \\
690.2 \mathrm{ft} \text { : Like } 689.6 \text { but finer grained, deformed. } \\
692.9,694.1 \mathrm{ft} \text { : Fine-grained carbonate. } \\
4.92 \mathrm{~kg} \text { of rock was processed. }\end{array}$ \\
\hline \multirow[t]{2}{*}{$\begin{array}{l}140 \\
\text { EMA (Red Dog } \\
\text { plate) }\end{array}$} & \multirow[t]{2}{*}{$\begin{array}{l}\text { De Long Mtns. } \\
\text { A-2 } \\
68^{\circ} 04^{\prime} 55^{\prime \prime} / \\
162^{\circ} 49^{\prime} 55^{\prime \prime}\end{array}$} & $\begin{array}{l}1 \text { indet. bar or blade fragment } \\
\text { CAI }=\sim 1.5 \\
\text { [DDH } 580 \text { (Paalaaq deposit), 576- } 77 \mathrm{ft}]\end{array}$ & Middle Ordovician-Triassic. & $\begin{array}{l}\text { Indeterminate (too few } \\
\text { conodonts). }\end{array}$ & $\begin{array}{l}\text { Fault-bounded section of Kivalina unit, } 537 \mathrm{ft} \text { thick; } \\
\text { sample of thin limy layer } 20 \mathrm{ft} \text { above base. } \\
\text { Light-gray, very fine grained limestone. Thin section is } \\
\text { deformed calcisiltite, cut by numerous irregular } \\
\text { stylolites and sparry calcite veins; } 2-5 \% \text { quartz silt. } \\
1.2 \mathrm{~kg} \text { of rock was processed. }\end{array}$ \\
\hline & & $\begin{array}{l}\text { Barren } \\
\text { [DDH 580, 592-93 ft] }\end{array}$ & & & $\begin{array}{l}\text { Sample } 5 \mathrm{ft} \text { above base of } 537-\mathrm{ft} \text { Kivalina unit section } \\
\text { and } 11 \mathrm{ft} \text { below top of } 16-\mathrm{ft} \text {-thick limestone layer. } \\
\text { Light-gray, very fine grained limestone with possible } \\
\text { peloids. Thin section is very finely crystalline } \\
\text { carbonate (mostly dolomite?) with vague pelloidal } \\
\text { texture, minor quartz silt, and trace phosphatic } \\
\text { bioclasts. } \\
\text { Heavy-mineral concentrate includes fluorite(?). } \\
1.0 \mathrm{~kg} \text { of rock was processed. }\end{array}$ \\
\hline
\end{tabular}


Table 2. Conodont samples from the Kivalina unit of the Kuna Formation-Continued.

[All faunas identified by A.G. Harris. Structural units determined by L.E. Young. EMA, Endicott Mountains allochthon. CAI, conodont color alteration index. Letters in field number refer to collector: AD, J.A. Dumoulin; and Cx, S.M. Curtis. DDH, diamond drill hole. Interval thicknesses in drill holes are drilled thicknesses and have not been corrected for dip of bedding. All drill hole samples collected by J.A. Dumoulin and (or) A.G. Harris unless otherwise indicated. Lithologic data under remarks are field descriptions unless otherwise indicated; thin section observations by J.A. Dumoulin. No., number; indet., indeterminate; loc., locality]

\begin{tabular}{|c|c|c|c|c|c|}
\hline $\begin{array}{l}\text { Locality No., } \\
\text { structural unit }\end{array}$ & $\begin{array}{l}\text { Quadrangle, } \\
\text { latitude n./ } \\
\text { longitude w. }\end{array}$ & $\begin{array}{c}\text { Conodont fauna and CAI } \\
\text { [field No.; USGS collection No.] }\end{array}$ & Age & $\begin{array}{c}\text { Conodont biofacies and } \\
\text { depositional } \\
\text { environment }\end{array}$ & Remarks \\
\hline $\begin{array}{l}141 \\
\text { EMA (Red Dog } \\
\text { plate) }\end{array}$ & $\begin{array}{l}\text { De Long Mtns. } \\
\text { A-2 } \\
68^{\circ} 04^{\prime} 54.6^{\prime \prime} / \\
162^{\circ} 49^{\prime} 32.4^{\prime \prime}\end{array}$ & $\begin{array}{l}\text { Barren. No conodonts or other mineralized fossil } \\
\text { materials were found. } \\
\text { [DDH } 710 \text { (Paalaaq deposit), composite conodont } \\
\text { sample: } 346-357.6 \mathrm{ft} \text { ] }\end{array}$ & & & $\begin{array}{l}\text { Sample from middle part of } \sim 670 \mathrm{ft} \text { thick highly } \\
\text { deformed interval of Kivalina unit, } \sim 77 \mathrm{ft} \text { above } \\
\text { altered ash layer. } \\
346-357.6 \mathrm{ft} \text { : Interbedded dark-gray to grayish-black } \\
\text { (outer), dark-gray to black (inner) mudstone and } \\
\text { light- to medium-light-gray (outer) carbonate } \\
\text { (mostly micrite, lesser silt- and fine-sand size). } \\
\text { Interbedded chiefly on mm to cm scale, but } \\
\text { carbonate layers up to } 3 \mathrm{~cm} \text { thick. Interval includes } \\
\sim 10 \% \text { calcite spar veins and } 1 \text { to } 3 \% \text { fine calcite } \\
\text { veins. Black mud(?) rip-up clasts to } 3 \mathrm{~cm} \text { long. } \\
\text { Thin section data: } \\
346.9 \mathrm{ft} \text { : Thin laminae of micrite, noncarbonate mud, } \\
\text { and carbonate sand (micrite clasts to } 2 \text { mm) with } \\
\text { calcareous spicules, and rare radiolarians. } \\
348.6 \mathrm{ft}: \text { Calcareous, dolomitic(?) radiolarite, some } \\
\text { radiolarians are pyritized. } \\
\text { Heavy-mineral concentrate includes rare pyritized } \\
\text { radiolarian steinkerns. } \\
5.86 \mathrm{~kg} \text { of rock was processed. }\end{array}$ \\
\hline $\begin{array}{l}142 \\
\text { EMA (Red Dog } \\
\text { plate) }\end{array}$ & $\begin{array}{l}\text { De Long Mtns. } \\
\text { A-2 } \\
68^{\circ} 04^{\prime} 54.3^{\prime \prime} / \\
162^{\circ} 49^{\prime} 24.3^{\prime \prime}\end{array}$ & $\begin{array}{l}\text { Scaliognathus anchoralis Branson and Mehl } \\
2 \text { Sa elements and } 3 \text { bar fragments } \\
\text { (="Hindeodella" segaformis Bischoff s.f.) [pl. 1, } \\
\text { figs. } 9,10] \\
\text { Unassigned elements: } \\
2 \mathrm{~Pb}(2 \text { morphotypes) and } 2 \mathrm{M} \\
6 \text { indet. bar and blade fragments } \\
\text { CAI=3.5 } \\
\text { [DDH } 640 \text { (Paalaaq deposit), composite conodont } \\
\text { sample: } 429-453 \mathrm{ft} ; 33488-\mathrm{PC}]\end{array}$ & $\begin{array}{l}\text { middle late Early } \\
\text { Mississippian (middle } \\
\text { Osagean; Sc. anchoralis- } \\
\text { Do. latus Zone). }\end{array}$ & $\begin{array}{l}\text { Indeterminate (too few } \\
\text { generically identifiable } \\
\text { conodonts). Conodonts } \\
\text { present indicate } \\
\text { postmortem transport } \\
\text { to a slope or deeper } \\
\text { water depositional } \\
\text { setting. }\end{array}$ & $\begin{array}{l}\text { Sample from 34-ft-thick section of Kivalina unit } \\
\text { bounded above and below by mélange, within } \sim 750 \\
\mathrm{ft} \text { thick interval of deformed Kivalina. } \\
429-453 \mathrm{ft} \text { : Light- to medium-gray (outer), medium- } \\
\text { dark-gray (inner), amalgamated, graded, fine- to } \\
\text { medium-grained carbonate turbidite beds } 6 \text { to } 8 \mathrm{~cm} \\
\text { thick, interbedded with noncalcareous grayish-black } \\
\text { (outer), grayish black to black (inner) mudstone. } \\
\text { Limestone is in discrete beds and forms } 30-35 \% \text { of } \\
\text { interval. Bedding near vertical in lower half of } \\
\text { sampled interval. Thin section at } 640.5 \mathrm{ft} \text { is sparse } \\
\text { calcareous radiolarite with brown muddy clasts and } \\
\text { local patches of fine-grained silica. } \\
6.0 \mathrm{~kg} \text { of rock was processed. }\end{array}$ \\
\hline
\end{tabular}


Table 2. Conodont samples from the Kivalina unit of the Kuna Formation-Continued.

[All faunas identified by A.G. Harris. Structural units determined by L.E. Young. EMA, Endicott Mountains allochthon. CAI, conodont color alteration index. Letters in field number refer to collector: AD, J.A. Dumoulin; and Cx, S.M. Curtis. DDH, diamond drill hole. Interval thicknesses in drill holes are drilled thicknesses and have not been corrected for dip of bedding. All drill hole samples collected by J.A. Dumoulin and (or) A.G. Harris unless otherwise indicated. Lithologic data under remarks are field descriptions unless otherwise indicated; thin section observations by J.A. Dumoulin. No., number; indet., indeterminate; loc., locality]

\begin{tabular}{|c|c|c|c|c|c|}
\hline $\begin{array}{l}\text { Locality No., } \\
\text { structural unit }\end{array}$ & $\begin{array}{l}\text { Quadrangle, } \\
\text { latitude n./ } \\
\text { longitude w. }\end{array}$ & $\begin{array}{c}\text { Conodont fauna and CAI } \\
\text { [field No.; USGS collection No.] }\end{array}$ & Age & $\begin{array}{c}\text { Conodont biofacies and } \\
\text { depositional } \\
\text { environment }\end{array}$ & Remarks \\
\hline \multirow[t]{2}{*}{$\begin{array}{l}143 \\
\text { EMA (Red Dog } \\
\text { plate) }\end{array}$} & \multirow[t]{2}{*}{$\begin{array}{l}\text { De Long Mtns. } \\
\text { A-2 } \\
68^{\circ} 04^{\prime} 50 " / \\
162^{\circ} 49^{\prime} 24.4^{\prime \prime}\end{array}$} & $\begin{array}{l}4 \text { Pa element fragments Bispathodus utahensis } \\
\text { Sandberg and Gutschick } \\
1 \text { Kladognathus sp. indet. bar fragment } \\
11 \text { indet. bar, blade, and platform fragments } \\
\text { CAI= 2.5 or } 3 \\
\text { [DDH } 636 \text { (Paalaaq deposit), } 534 \mathrm{ft} ; 33422-\mathrm{PC}]\end{array}$ & $\begin{array}{l}\text { Early-early Late } \\
\text { Mississippian (late } \\
\text { Kinderhookian- } \\
\text { Meramecian) }\end{array}$ & $\begin{array}{l}\text { Indeterminate (too few } \\
\text { conodonts); conodonts } \\
\text { present indicate outer } \\
\text { shelf or deeper water } \\
\text { depositional setting. }\end{array}$ & $\begin{array}{l}\text { Fault-bounded section of Kivalina unit, } 44 \mathrm{ft} \text { thick; } \\
\text { sample } 10 \mathrm{ft} \text { below top. } \\
\text { Sample is 6-cm-thick layer of light-gray-weathering, } \\
\text { fine- to medium-grained limestone (skeletal } \\
\text { packstone to grainstone with abundant crinoids). } \\
\text { Thin section is packed skeletal grainstone; bioclasts } \\
\text { mostly crinoid ossicles (to } 1.5 \mathrm{~mm} \text { ) and lesser } \\
\text { foraminifers, bryozoan fragments, and rare ostracode } \\
\text { valves. Pyrite replaces some skeletal grains. } \\
\text { Heavy-mineral concentrate includes minor pyritized } \\
\text { bioclasts (mainly bryozoans). } \\
0.2 \text { kg of rock was processed. }\end{array}$ \\
\hline & & $\begin{array}{l}\text { Barren } \\
\text { [DDH 636, 536-37 ft] }\end{array}$ & & & $\begin{array}{l}\text { Sample } 13 \mathrm{ft} \text { below top of } 44-\mathrm{ft}-\text { thick, fault-bounded } \\
\text { section of Kivalina unit. } \\
\text { Interval of light-gray, very fine grained, silty(?) } \\
\text { limestone layers }(0.5-8 \mathrm{~cm} \text { thick) interbedded with } \\
\text { dark-gray limy shale. Three thin sections taken from } \\
\text { limestone layers in this interval. Two are white } \\
\text { sparry calcite with cone-in-cone structure; the third } \\
\text { is graded calcisiltite to calcisandstone with flattened } \\
\text { noncalcareous mud intraclasts and rare identifiable } \\
\text { bioclasts (pelmatozoan and bryozoan fragments, } \\
\text { calcareous spicules and calcitized radiolarians?). } \\
\text { Trace quartz silt. } \\
0.5 \text { kg of rock was processed. } \\
\end{array}$ \\
\hline $\begin{array}{l}145 \\
\text { EMA (Red Dog } \\
\text { plate) }\end{array}$ & $\begin{array}{l}\text { De Long Mtns. } \\
\text { A-2 } \\
68^{\circ} 04^{\prime} 34^{\prime \prime} / \\
162^{\circ} 49^{\prime} 28.5^{\prime \prime}\end{array}$ & $\begin{array}{l}\text { Barren. No conodonts or other mineralized fossil } \\
\text { materials were found. } \\
\text { [DDH } 495 \text { (Aqqaluk deposit), composite conodont } \\
\text { sample 1: } 488-488.3 \text { and } 493-495.7 \mathrm{ft} \text { ] }\end{array}$ & & & $\begin{array}{l}\text { Sample of Kivalina unit } \sim 5 \mathrm{ft} \text { below mélange zone at } \\
\text { contact with overlying Ikalukrok unit. } \\
488.0-495.7 \mathrm{ft} \text { : Laminated to very thin bedded }(0.1-1 \\
\mathrm{cm} \text { ) light-gray to medium-dark-gray (outer), } \\
\text { medium-dark-gray and grayish-black (inner) } \\
\text { limestone and subordinate }(<5 \text { to } 10 \%) \text { mudstone } \\
\text { with }<5 \% \text { calcite spar veins. Coarsest layers are } \\
\text { medium-grained skeletal supportstone with } \\
\text { pelmatozoan debris. Rare mud rip-up clasts to } 4 \mathrm{~cm} \\
\text { long. Thin section at } 493.5 \mathrm{ft} \text { has bioclasts and } \\
\text { peloids in deformed, laminated matrix of } \\
\text { noncarbonate and carbonate mud. } \\
4.94 \mathrm{~kg} \text { of rock was processed. }\end{array}$ \\
\hline
\end{tabular}


Table 2. Conodont samples from the Kivalina unit of the Kuna Formation-Continued.

[All faunas identified by A.G. Harris. Structural units determined by L.E. Young. EMA, Endicott Mountains allochthon. CAI, conodont color alteration index. Letters in field number refer to collector: AD, J.A. Dumoulin; and Cx, S.M. Curtis. DDH, diamond drill hole. Interval thicknesses in drill holes are drilled thicknesses and have not been corrected for dip of bedding. All drill hole samples collected by J.A. Dumoulin and (or) A.G. Harris unless otherwise indicated. Lithologic data under remarks are field descriptions unless otherwise indicated; thin section observations by J.A. Dumoulin. No., number; indet., indeterminate; loc., locality]

\begin{tabular}{|c|c|c|c|c|c|}
\hline $\begin{array}{l}\text { Locality No., } \\
\text { structural unit }\end{array}$ & $\begin{array}{l}\text { Quadrangle, } \\
\text { latitude n./ } \\
\text { longitude w. }\end{array}$ & $\begin{array}{c}\text { Conodont fauna and CAI } \\
\text { [field No.; USGS collection No.] }\end{array}$ & Age & $\begin{array}{c}\text { Conodont biofacies and } \\
\text { depositional } \\
\text { environment }\end{array}$ & Remarks \\
\hline $\begin{array}{l}145 \\
\text { EMA (Red Dog } \\
\text { plate) } \\
\text { [cont.] }\end{array}$ & $\begin{array}{l}\text { De Long Mtns. } \\
\text { A-2 } \\
68^{\circ} 04^{\prime} 34^{\prime \prime} / \\
162^{\circ} 49^{\prime} 28.5^{\prime \prime}\end{array}$ & $\begin{array}{l}\text { Barren. No conodonts or other mineralized fossil } \\
\text { materials were found. } \\
\text { [DDH 495, composite conodont sample 2: 510.4- } \\
511.0,511.5-513 \text {, and } 514-516 \mathrm{ft}]\end{array}$ & & & $\begin{array}{l}\text { Sample of Kivalina unit } 27 \mathrm{ft} \text { below mélange zone at } \\
\text { contact with overlying Ikalukrok unit. } \\
510.4-516.1 \mathrm{ft} \text { : Laminated to irregularly laminated very } \\
\text { thin beds }(1-3 \mathrm{~mm} \text { ) of light- to medium-dark-gray } \\
\text { (outer) and medium-dark- to dark-gray (inner) } \\
\text { limestone with }<5 \% \text { mudstone. Coarsest layers (to } 2 \\
\mathrm{~cm} \text { thick) are medium-grained with dark mud(?) } \\
\text { clasts. Thin section at } 516.4 \mathrm{ft} \text { is calcareous spiculite } \\
\text { with micrite clasts and quartz silt and sand. } \\
\text { Heavy-mineral concentrate contains rare sphalerite(?). } \\
5.76 \mathrm{~kg} \text { of rock was processed. }\end{array}$ \\
\hline $\begin{array}{l}146 \\
\text { EMA (Red Dog } \\
\text { plate) }\end{array}$ & $\begin{array}{l}\text { De Long Mtns. } \\
\text { A-2 } \\
68^{\circ} 04^{\prime} 33.7^{\prime \prime} / \\
162^{\circ} 49^{\prime} 20.7^{\prime \prime}\end{array}$ & $\begin{array}{l}\text { Barren. No conodonts or other mineralized fossil } \\
\text { materials were found. } \\
\text { [DDH } 499 \text { (Aqqaluk deposit), composite conodont } \\
\text { sample: } 447.8-457 \mathrm{ft} \text { ] }\end{array}$ & & & $\begin{array}{l}\text { Sample of Kivalina unit } 3 \mathrm{ft} \text { below gradational contact } \\
\text { with mineralized zone at base of Ikalukrok unit. } \\
447.8-457.0 \mathrm{ft} \text { : Various proportions of laminated to } \\
\text { very thin bedded }(0.1-0.5 \mathrm{~cm} \text { ), medium-light- to } \\
\text { light-gray (outer), medium-dark-gray (inner) } \\
\text { limestone, calcareous mudstone, and medium- to } \\
\text { dark-gray (outer) and medium-dark-gray (inner) } \\
\text { mudstone with } 1-2 \% \text { calcite spar veins. Coarsest } \\
\text { layers are fine to very fine grained, with } \\
\text { pelmatozoan(?) fragments. } \\
\text { Thin section data: } \\
447 \mathrm{ft} \text { : Fine-grained argillaceous rock with deformed } \\
\text { (flattened) fabric. } \\
454.2 \mathrm{ft} \text { : Thin layer of fine-grained carbonate with } \\
\text { pyritized bioclasts. } \\
6.0 \mathrm{~kg} \text { of rock was processed. }\end{array}$ \\
\hline
\end{tabular}


Table 2. Conodont samples from the Kivalina unit of the Kuna Formation-Continued.

[All faunas identified by A.G. Harris. Structural units determined by L.E. Young. EMA, Endicott Mountains allochthon. CAI, conodont color alteration index. Letters in field number refer to collector: AD, J.A. Dumoulin; and Cx, S.M. Curtis. DDH, diamond drill hole. Interval thicknesses in drill holes are drilled thicknesses and have not been corrected for dip of bedding. All drill hole samples collected by J.A. Dumoulin and (or) A.G. Harris unless otherwise indicated. Lithologic data under remarks are field descriptions unless otherwise indicated; thin section observations by J.A. Dumoulin. No., number; indet., indeterminate; loc., locality]

\begin{tabular}{|c|c|c|c|c|c|}
\hline $\begin{array}{l}\text { Locality No., } \\
\text { structural unit }\end{array}$ & $\begin{array}{l}\text { Quadrangle, } \\
\text { latitude } \mathrm{n} . / \\
\text { longitude w. }\end{array}$ & $\begin{array}{c}\text { Conodont fauna and CAI } \\
\text { [field No.; USGS collection No.] }\end{array}$ & Age & $\begin{array}{c}\text { Conodont biofacies and } \\
\text { depositional } \\
\text { environment }\end{array}$ & Remarks \\
\hline $\begin{array}{l}153 \\
\text { EMA (Red Dog } \\
\text { plate) }\end{array}$ & $\begin{array}{l}\text { De Long Mtns. } \\
\text { A-2 } \\
68^{\circ} 04^{\prime} 20.2^{\prime \prime} / \\
162^{\circ} 49^{\prime} 31.4^{\prime \prime}\end{array}$ & $\begin{array}{l}\text { Barren. No conodonts or other mineralized fossil } \\
\text { materials were found. } \\
\text { [DDH } 415 \text { (Main deposit); composite conodont } \\
\text { sample: } 110-148.5 \mathrm{ft} \text { ] }\end{array}$ & & & $\begin{array}{l}\text { Sample from lower part of 80-ft-thick fault-bounded } \\
\text { section of Kivalina unit that overlies another faulted } \\
\text { section of Kivalina(?) that contains altered ash beds. } \\
\text { 110-148.5 ft: Various proportions of laminated to very } \\
\text { thin bedded (up to } 5 \mathrm{~cm} \text { ), light- to medium-light- } \\
\text { gray (outer) and medium-dark-gray (inner) } \\
\text { limestone and calcareous mudstone, and black } \\
\text { (outer) and dark-gray (inner) noncalcareous } \\
\text { mudstone, with } 10-30 \% \text { calcite spar veins; rare cross } \\
\text { laminations and graded beds. Coarsest layers are } \\
\text { medium- to coarse-grained skeletal packstone and } \\
\text { grainstone. } \\
\text { Thin section data: } \\
115,116.5 \text {, and } 124.5 \mathrm{ft} \text { : Bioclasts (mainly crinoid } \\
\text { ossicles, lesser spicules and calcispheres) and local } \\
\text { mud clasts in finely laminated matrix of carbonate } \\
\text { and noncarbonate mud; all samples look deformed. } \\
6.24 \text { kg of rock was processed. }\end{array}$ \\
\hline $\begin{array}{l}154 \\
\text { EMA (Red Dog } \\
\text { plate) }\end{array}$ & $\begin{array}{l}\text { De Long Mtns. } \\
\text { A-2 } \\
68^{\circ} 04^{\prime} 20.2^{\prime \prime} / \\
162^{\circ} 49^{\prime} 01.5^{\prime \prime}\end{array}$ & $\begin{array}{l}\text { Barren } \\
\text { [DDH } 8 \text { (Main deposit), 285-293.5 ft] }\end{array}$ & & & $\begin{array}{l}\text { Limestone and shale of the Kivalina unit, } \sim 8 \mathrm{ft} \text { below } \\
\text { mineralized horizon at base of Ikalukrok unit. See } \\
\text { table } 1 \text { for Ikalukrok sample from this drill hole. } \\
3.9 \mathrm{~kg} \text { of rock was processed. } \\
\text { Sample collected by D. Moore, I.L. Tailleur, and L.E. } \\
\text { Young. }\end{array}$ \\
\hline $\begin{array}{l}155 \\
\text { EMA (Red Dog } \\
\text { plate) }\end{array}$ & $\begin{array}{l}\text { De Long Mtns. } \\
\text { A-2 } \\
68^{\circ} 04^{\prime} 14.7 " / \\
162^{\circ} 49^{\prime} 37.7^{\prime \prime}\end{array}$ & $\begin{array}{l}\text { Barren } \\
\text { [DDH } 77 \text { (Main deposit), 364-391 ft] }\end{array}$ & & & $\begin{array}{l}\text { Silicified shale and limestone of the Kivalina unit, just } \\
\text { below sulfide mineralization at base of Ikalukrok } \\
\text { unit; contains sphalerite and pyrite veins. } \\
8.4 \mathrm{~kg} \text { of rock was processed. } \\
\text { Sample collected by D. Moore, I.L. Tailleur, and L.E. } \\
\text { Young. }\end{array}$ \\
\hline
\end{tabular}


Table 3. Conodont samples from the Kuna Formation

[All faunas identified by A.G. Harris. Structural units determined by L.E. Young. EMA, Endicott Mountains allochthon. CAI, conodont color alteration index. Letters in field number refer to collector: AD, J.A. Dumoulin; AK, J.S. Kelley; and MD, C.F. Mayfield. DDH, diamond drill hole. Interval thicknesses in drill holes are drilled thicknesses and have not been corrected for dip of bedding. Lithologic data under remarks are field descriptions unless otherwise indicated; thin section observations by J.A. Dumoulin. No., number; indet., indeterminate; loc., locality]

\begin{tabular}{|c|c|c|c|c|c|}
\hline $\begin{array}{l}\text { Locality No., } \\
\text { structural unit }\end{array}$ & $\begin{array}{l}\text { Quadrangle, } \\
\text { latitude } \mathrm{n} . / \\
\text { longitude w. }\end{array}$ & $\begin{array}{c}\text { Conodont fauna and CAI } \\
\text { [field No.; USGS collection No.] }\end{array}$ & Age & $\begin{array}{c}\text { Conodont biofacies and } \\
\text { depositional } \\
\text { environment }\end{array}$ & Remarks \\
\hline $\begin{array}{l}24 \\
\text { EMA (Key } \\
\text { Creek plate) }\end{array}$ & $\begin{array}{l}\text { De Long Mtns. } \\
\text { A-3 } \\
68^{\circ} 07^{\prime} 08^{\prime \prime} / \\
163^{\circ} 17^{\prime} 14^{\prime \prime}\end{array}$ & $\begin{array}{l}\text { All conodonts are partly or completely coated with } \\
\text { organic matter. } \\
\text { Bispathodus utahensis Sandberg and Gutschick } \\
26 \mathrm{~Pa}, 3 \mathrm{~Pb}, 3 \mathrm{M}, 3 \mathrm{Sa} \text {, and } 2 \text { Sb elements } \\
\text { Idioprioniodus conjunctus } \text { (Gunnell) } \\
4 \mathrm{~Pa}, 1 \mathrm{M} \text {, and } 1 \mathrm{Sc} \text { element fragments } \\
\text { Kladognathus tenuis (Branson and Mehl) } \\
3 \mathrm{~Pa}-\mathrm{Pb}, 2 \mathrm{M} \text {, and } 6 \text { Sb-Sc elements } \\
1 \mathrm{Sc} \text { element Scaliognathus anchoralis Branson } \\
\text { and Mehl } \\
\text { Unassigned elements: } \\
2 \mathrm{~Pb}(2 \text { morphotypes) and } 2 \mathrm{Sc} \\
199 \text { indet. bar, blade, and platform fragments } \\
\text { CAI= 3 } \\
\text { [00AD24A; 33635-PC] }\end{array}$ & $\begin{array}{l}\text { middle late Early } \\
\text { Mississippian (middle } \\
\text { Osagean; Sc. anchoralis-Do. } \\
\text { latus Zone). }\end{array}$ & $\begin{array}{l}\text { Bispathodid-digyrate } \\
\text { biofacies: conodonts } \\
\text { indicate a basinal } \\
\text { depositional setting. }\end{array}$ & $\begin{array}{l}113.5 \mathrm{ft} \text { below top of Kuna Formation at IP Creek. } \\
\text { Very pale orange- to grayish-orange-weathering, } \\
\text { pinkish to medium-dark-gray, finely sucrosic, blocky } \\
\text { weathering dolostone. Thin section is organic-rich } \\
\text { fine-crystalline dolostone. } \\
13.1 \mathrm{~kg} \text { of rock was processed. } \\
\text { Loc. } 12 \text { (fig. 2) of Dumoulin and others (2004). }\end{array}$ \\
\hline $\begin{array}{l}103 \\
\text { EMA (Key } \\
\text { Creek plate) }\end{array}$ & $\begin{array}{l}\text { De Long Mtns. } \\
\text { B-1 } \\
68^{\circ} 21^{\prime} 21^{\prime \prime} / \\
162^{\circ} 02^{\prime} 16^{\prime \prime}\end{array}$ & $\begin{array}{l}1 \text { juvenile Pa element Eotaphrus sp. indet. } \\
3 \text { Pa elements Polygnathus communis Branson and } \\
\text { Mehl [pl. 1, fig. 14] } \\
\text { Scaliognathus anchoralis Branson and Mehl: } \\
3 \mathrm{Sc} \text { and } 31 \text { bar fragments [pl. 1, fig. 15] } \\
\text { Unassigned elements: } \\
10 \text { juvenile } \mathrm{Pa}, 4 \mathrm{~Pb} \text { (4 morphotypes), } 2 \mathrm{M}(2 \\
\text { morphotypes), } 1 \mathrm{Sa} \text {, and } 3 \mathrm{Sb} \text { (3 morphotypes) } \\
52 \text { indet. bar, blade, and platform fragments } \\
\mathrm{CAI}=2 \\
{[00 \mathrm{AD} 17 \mathrm{H} ; 33631-\mathrm{PC}]}\end{array}$ & $\begin{array}{l}\text { middle late Early } \\
\text { Mississippian (middle } \\
\text { Osagean; Sc. anchoralis-Do. } \\
\text { latus Zone). }\end{array}$ & $\begin{array}{l}\text { Postmortem transport } \\
\text { within or from a slope } \\
\text { depositional setting } \\
\text { with minor shallow } \\
\text { water components } \\
\text { (Eotaphrus sp.). }\end{array}$ & $\begin{array}{l}\text { Sample from middle(?) part of Kuna Formation at } \\
\text { August Creek; see table } 11 \text { for Kayak Shale sample } \\
\text { from this locality. } \\
\text { Medium-light-gray- and medium-gray-weathering, } \\
\text { medium-gray to medium-dark-gray, fine to very fine } \\
\text { grained limestone in beds } 3 \text { to } 8 \mathrm{~cm} \text { thick with 10- } \\
20 \% \text { chert. Thin-section is a calcareous spiculite. } \\
10.2 \mathrm{~kg} \text { of rock was processed. } \\
\text { Loc. } 2 \text { (app. 1) of Dumoulin and others (2004). }\end{array}$ \\
\hline
\end{tabular}


Table 3. Conodont samples from the Kuna Formation-Continued.

[All faunas identified by A.G. Harris. Structural units determined by L.E. Young. EMA, Endicott Mountains allochthon. CAI, conodont color alteration index. Letters in field number refer to collector: AD, J.A. Dumoulin; AK, J.S. Kelley; and MD, C.F. Mayfield. DDH, diamond drill hole. Interval thicknesses in drill holes are drilled thicknesses and have not been corrected for dip of bedding. Lithologic data under remarks are field descriptions unless otherwise indicated; thin section observations by J.A. Dumoulin. No., number; indet., indeterminate; loc., locality]

\begin{tabular}{|c|c|c|c|c|c|}
\hline $\begin{array}{l}\text { Locality No., } \\
\text { structural unit }\end{array}$ & $\begin{array}{l}\text { Quadrangle, } \\
\text { latitude n./ } \\
\text { longitude w. }\end{array}$ & $\begin{array}{c}\text { Conodont fauna and CAI } \\
\text { [field No.; USGS collection No.] }\end{array}$ & Age & $\begin{array}{c}\text { Conodont biofacies and } \\
\text { depositional } \\
\text { environment }\end{array}$ & Remarks \\
\hline $\begin{array}{l}103 \\
\text { [cont.] }\end{array}$ & $\begin{array}{l}\text { De Long Mtns. } \\
\text { B-1 } \\
68^{\circ} 21^{\prime} 21^{\prime \prime} / \\
162^{\circ} 02^{\prime} 16^{\prime \prime}\end{array}$ & $\begin{array}{l}\text { Pa element fragments Bispathodus utahensis } \\
\text { Sandberg and Gutschick } \\
1 \text { juvenile Pa element Dollymae hassi Voges? } \\
2 \text { Pa elements Doliognathus latus Branson and } \\
\text { Mehl } \\
6 \text { P elements Eotaphrus burlingtonensis Pierce and } \\
\text { Langenheim [pl. 1, figs. 11-13] } \\
2 \text { Pa elements Gnathodus cuneiformis Mehl and } \\
\text { Thomas [pl. 1, figs. 18, 19] } \\
\text { 32 Sb \& Sc element fragments Scaliognathus } \\
\text { anchoralis and (or) Doliognathus latus } \\
15 \text { Pa elements Polygnathus communis communis } \\
\text { Branson and Mehl } \\
\text { Unassigned elements: } \\
\text { 13 Pb (2 morphotypes, most are probably in the } \\
\text { apparatus of D. latus) and 17 M (3 morphotypes) } \\
\sim 100 \text { indet. bar, blade, and platform fragments } \\
\text { CAI=3 } \\
\text { [79MD44C; 27506-PC] }\end{array}$ & $\begin{array}{l}\text { middle late Early } \\
\text { Mississippian (middle } \\
\text { Osagean; } S c \text {. anchoralis-D. } \\
\text { latus Zone). } \\
\text { This sample correlates with } \\
\text { 92AD35 (31781-PC) from } \\
\text { near the base of the type } \\
\text { section of the Kuna } \\
\text { Formation in the Howard } \\
\text { Pass B-3 quadrangle. } \\
\text { Sample 92AD35, however, } \\
\text { is biostratigraphically more } \\
\text { diagnostic and indicates the } \\
\text { lower part of the } S c \text {. } \\
\text { anchoralis-Do. latus Zone. }\end{array}$ & $\begin{array}{l}\text { Mixed biofacies. } \\
\text { Diverse assemblage of } \\
\text { both shallow-water }(E \text {. } \\
\text { burlingtonensis) and } \\
\text { deep-water }(D \text {. hassi }) \\
\text { forms. Overall species } \\
\text { association indicates } \\
\text { outer shelf or slope } \\
\text { depositional setting. }\end{array}$ & $\begin{array}{l}\text { Sample from lower 1-m-thick carbonate zone in Kuna } \\
\text { Formation; stratigraphic position relative to } \\
\text { 00AD17H is uncertain. } \\
4.7 \mathrm{~kg} \text { of rock was processed. } \\
\text { Fossil loc. 11, Curtis and others (1990). }\end{array}$ \\
\hline $\begin{array}{l}106 \\
\text { EMA (Key } \\
\text { Creek plate) }\end{array}$ & $\begin{array}{l}\text { De Long Mtns. } \\
\text { B-1 } \\
68^{\circ} 16^{\prime} 04^{\prime \prime} / \\
162^{\circ} 09^{\prime} 27.5^{\prime \prime}\end{array}$ & $\begin{array}{l}1 \text { digyrate Pb element } \\
5 \text { indet. bar and blade fragments } \\
\mathrm{CAI}=3 \\
{[97 \mathrm{AK} 59 \mathrm{C}]}\end{array}$ & post-Devonian Paleozoic & $\begin{array}{l}\text { Indeterminate (too few } \\
\text { generically identifiable } \\
\text { conodonts). }\end{array}$ & $\begin{array}{l}\text { Sample taken near base of Kuna Formation, directly } \\
\text { above contact with Kayak Shale, from limestone } \\
\text { interval a few } \mathrm{ft} \text { thick. } \\
\text { Flaggy, sooty dark-gray limestone in beds } 3 \text { to } 5 \mathrm{~cm} \\
\text { thick (turbidites?). Thin section is calcareous spiculite } \\
\text { (a few spicules are siliceous) with rare calcitized } \\
\text { radiolarians(?). Matrix is noncalcareous and } \\
\text { calcareous mud with dolomite rhombs and trace } \\
\text { monocrystalline quartz. } \\
\text { Heavy-mineral concentrate is chiefly pyritized spine } \\
\text { fillings and (or) spicules. } \\
13.6 \mathrm{~kg} \text { of rock was processed. } \\
\text { Shale } 20 \mathrm{ft} \text { above limestone interval yielded common } \\
\text { small circular spores and scolecodonts and rare } \\
\text { ?densospore rim fragments of possible Carboniferous } \\
\text { age (H. Haga, unpublished fossil report, 1997). }\end{array}$ \\
\hline
\end{tabular}


Table 3. Conodont samples from the Kuna Formation-Continued.

[All faunas identified by A.G. Harris. Structural units determined by L.E. Young. EMA, Endicott Mountains allochthon. CAI, conodont color alteration index. Letters in field number refer to collector: AD, J.A. Dumoulin; AK, J.S. Kelley; and MD, C.F. Mayfield. DDH, diamond drill hole. Interval thicknesses in drill holes are drilled thicknesses and have not been corrected for dip of bedding. Lithologic data under remarks are field descriptions unless otherwise indicated; thin section observations by J.A. Dumoulin. No., number; indet., indeterminate; loc., locality]

\begin{tabular}{|c|c|c|c|c|c|}
\hline $\begin{array}{l}\text { Locality No., } \\
\text { structural unit }\end{array}$ & $\begin{array}{l}\text { Quadrangle, } \\
\text { latitude n./ } \\
\text { longitude w. }\end{array}$ & $\begin{array}{c}\text { Conodont fauna and CAI } \\
\text { [field No.; USGS collection No.] }\end{array}$ & Age & $\begin{array}{c}\text { Conodont biofacies and } \\
\text { depositional } \\
\text { environment }\end{array}$ & Remarks \\
\hline \multirow[t]{2}{*}{$\begin{array}{l}107 \\
\text { EMA (Key } \\
\text { Creek plate) }\end{array}$} & \multirow[t]{2}{*}{$\begin{array}{l}\text { De Long Mtns. } \\
\text { B-1 } \\
68^{\circ} 15^{\prime} 31.4^{\prime \prime} / \\
162^{\circ} 13^{\prime} 20^{\prime \prime}\end{array}$} & $\begin{array}{l}1 \text { Pa element fragment Gnathodus texanus } \\
\text { Roundy? } \\
12 \text { indet. bar and blade fragments } \\
\text { CAI }=3.5 \\
{[97 \mathrm{AK} 74 \mathrm{~A}]}\end{array}$ & $\begin{array}{l}\text { latest Early-middle Late } \\
\text { Mississippian (late Osagean- } \\
\text { early Chesterian). }\end{array}$ & $\begin{array}{l}\text { Indeterminate (too few } \\
\text { conodonts). Probably } \\
\text { open-marine } \\
\text { depositional setting. }\end{array}$ & $\begin{array}{l}\text { Part of } 158 \text {-ft measured section of upper Kuna } \\
\text { Formation and lower Etivluk Group, sampled chiefly } \\
\text { for radiolarians; see table } 14 \text { for additional samples } \\
\text { from this locality. Sample } \sim 5 \mathrm{ft} \text { above base of section } \\
\text { and } \sim 83 \mathrm{ft} \text { below contact with Etivluk. } \\
\text { Brown-gray carbonate (beds } \sim 5 \mathrm{~cm} \text { thick) interbedded } \\
\text { with chert. Thin section is fine-grained dolomite } \\
\text { (crystals } 20-100 \mu \mathrm{m} \text { ), micrite, and sparry calcite, with } \\
<1 \% \text { monocrystalline quartz, trace crinoid ossicles, } \\
\text { and irregular spar-filled fenestrae(?). } \\
\text { Heavy-mineral concentrate is chiefly barite, minor } \\
\text { dolomitized and baritized composite grains, and very } \\
\text { minor pink fluorite(?). } \\
6.5 \mathrm{~kg} \text { of rock was processed. } \\
\text { Loc. } 6 \text { (app. 1) of Dumoulin and others (2004). }\end{array}$ \\
\hline & & $\begin{array}{l}\text { Gnathodus texanus Roundy (adults and juveniles, } \\
\text { many complete): } \\
23 \mathrm{~Pa}, 3 \mathrm{~Pb}, 4 \mathrm{M} \text {, and } 3 \mathrm{Sc} \text { elements [pl. 1, fig. 17] } \\
1 \mathrm{M} \text { element Idioprioniodus sp. indet. } \\
6 \mathrm{~Pa} \text { elements (mostly fragments) } \\
\text { Rhachistognathus prolixus Baesemann and Lane } \\
\text { [pl. 1, fig. 16] } \\
\text { Unassigned elements: } \\
1 \mathrm{M} \text { and } 1 \text { Sc element } \\
213 \text { indet. bar, blade, and platform fragments } \\
\text { (overwhelmingly free blade fragments of, most } \\
\text { likely, G. texanus) } \\
\text { CAI=3.5 } \\
\text { [97AK74F; 33404-PC] }\end{array}$ & $\begin{array}{l}\text { middle Late Mississippian } \\
\text { (early Chesterian). }\end{array}$ & $\begin{array}{l}\text { G. texanus biofacies: } \\
\text { open-marine shelf or } \\
\text { deeper water } \\
\text { depositional } \\
\text { environment. Presence } \\
\text { of Rhachistognathus } \\
\text { suggests seaward } \\
\text { transport of high- } \\
\text { energy, shoal-water } \\
\text { forms. }\end{array}$ & $\begin{array}{l}\text { Sample } \sim 40 \mathrm{ft} \text { above } 97 \mathrm{AK} 74 \mathrm{~A} \text { and } \sim 44 \mathrm{ft} \text { below } \\
\text { contact with Etivluk Group. } \\
\text { Chert in nodular beds, } 2 \text { - to } 10 \text {-cm-thick, with shale } \\
\text { partings, overlain by medium-dark-gray, laminated, } \\
\text { fine-grained limestone. Thin section is chert with } \\
\text { abundant radiolarians and sponge spicules (siliceous, } \\
\text { calcareous, and pyritized). Radiolarians few to } 50 \% \\
\text { and graded(?); some have well-preserved test } \\
\text { structure. } \\
\text { Heavy-mineral concentrate is chiefly baritized } \\
\text { composite grains (including chert) and conodonts. } \\
7.1 \text { kg of rock was processed. }\end{array}$ \\
\hline $\begin{array}{l}158 \\
\text { EMA (Key } \\
\text { Creek plate, } \\
\text { near transition } \\
\text { to Red Dog } \\
\text { plate) }\end{array}$ & $\begin{array}{l}\text { De Long Mtns. } \\
\text { A-2 } \\
68^{\circ} 05^{\prime} 8.2^{\prime \prime} / \\
162^{\circ} 45^{\prime} 37.1^{\prime \prime}\end{array}$ & $\begin{array}{l}\text { Nearly all the conodonts are partly or extensively } \\
\text { coated with gritty organic matter. } \\
2 \text { incomplete Pa elements Pseudopolygnathus sp. } \\
\text { Unassigned elements: } \\
1 \mathrm{M} \text { and } 1 \mathrm{~S} \\
18 \text { indet. bar, blade, and platform elements } \\
\mathrm{CAI}=\sim 2.5-3.5 \\
\text { [DDH } 587 \text { (east of Paalaaq deposit), } 134 \mathrm{ft} ; 33421 \text { - } \\
\text { PC] }\end{array}$ & Early Mississippian & $\begin{array}{l}\text { Indeterminate (too few } \\
\text { conodonts). }\end{array}$ & $\begin{array}{l}\text { 3-ft-thick turbidite bed in Kuna Formation; sample } \\
>100 \mathrm{ft} \text { above gradational contact with Kayak Shale. } \\
\text { See table } 11 \text { for Kayak sample from this locality. } \\
\text { Dark-gray, carbonaceous, shaly limestone with } \\
\text { calcitized radiolarians(?). Thin section is peloids, } \\
\text { lime mud intraclasts, calcareous spicules, and } \\
\text { calcitized radiolarians in a finely crystalline matrix of } \\
\text { calcite (and lesser dolomite?). } \\
0.4 \text { kg of rock was processed. } \\
\text { Sample collected by J.A. Dumoulin. }\end{array}$ \\
\hline
\end{tabular}


Table 4. Conodont samples from deep-water facies of the Lisburne Group in the Picnic Creek allochthon

[All faunas identified by A.G. Harris. Structural units determined by L.E. Young. PCA, Picnic Creek allochthon. CAI, conodont color alteration index. Letters in field number refer to collector unless otherwise indicated: AD, J.A. Dumoulin; EK, I. Ellersieck; JS, J.M. Schmidt; and KE, K. Evans. Lithologic data under remarks are field descriptions unless otherwise indicated; thin section observations by J.A. Dumoulin. No., number; indet., indeterminate; loc., locality]

\begin{tabular}{|c|c|c|c|c|c|}
\hline $\begin{array}{l}\text { Locality No., } \\
\text { structural unit }\end{array}$ & $\begin{array}{l}\text { Quadrangle, } \\
\text { latitude n./ } \\
\text { longitude w. }\end{array}$ & $\begin{array}{c}\text { Conodont fauna and CAI } \\
\text { [field No.; USGS collection No.] }\end{array}$ & Age & $\begin{array}{c}\text { Conodont biofacies and } \\
\text { depositional } \\
\text { environment } \\
\end{array}$ & Remarks \\
\hline \multirow[t]{2}{*}{$\begin{array}{l}7 \\
\text { PCA (Amaruk } \\
\text { plate) }\end{array}$} & \multirow[t]{2}{*}{\begin{tabular}{|l|} 
De Long Mtns. \\
B-3 \\
$68^{\circ} 18^{\prime} 11.8^{\prime \prime} /$ \\
$163^{\circ} 14^{\prime} 9.5^{\prime \prime}$
\end{tabular}} & $\begin{array}{l}\text { Barren } \\
{[\text { KE98-16B] }}\end{array}$ & & & $\begin{array}{l}\text { Sample } 100 \mathrm{ft} \text { below highest exposed Lisburne Group. } \\
\text { Light-gray-weathering, very light gray, laminated } \\
\text { dolostone, partly siliceous and locally interbedded } \\
\text { with chert; some grainy (bioclastic?) layers. Thin } \\
\text { section is euhedral dolomite mosaic with minor } \\
\text { intercrystal silica; some vugs contain dead oil(?). } \\
\text { Heavy-mineral concentrate includes rare siliceous } \\
\text { single and double-axon sponge spicules. } \\
5.42 \text { kg of rock was processed. } \\
\text { Loc. } 2 \text { (fig. 2) of Dumoulin and others (2004). }\end{array}$ \\
\hline & & $\begin{array}{l}\text { Barren } \\
{[\text { KE98-16C] }}\end{array}$ & & & $\begin{array}{l}\text { Sample } \sim 80 \mathrm{ft} \text { below KE98-16B. } \\
\text { Brown, laminated dolostone and mudstone } \\
\text { (turbidites?). Thin section is dark, noncalcareous } \\
\text { mudstone with scattered siliceous sponge spicules } \\
\text { and rare, small, carbonate bioclast fragments. } \\
3.2 \mathrm{~kg} \text { of rock was processed. }\end{array}$ \\
\hline $\begin{array}{l}10 \\
\text { PCA (Amaruk } \\
\text { plate) }\end{array}$ & $\begin{array}{l}\text { De Long Mtns. } \\
\text { A-3 } \\
68^{\circ} 10^{\prime} 34.7^{\prime \prime /} \\
163^{\circ} 34^{\prime} 31.8^{\prime \prime}\end{array}$ & $\begin{array}{l}2 \text { Pa elements Polygnathus cf. Po. communis } \\
\text { Branson and Mehl (both partly covered with } \\
\text { adventitious quartz silt) } \\
4 \text { indet. bar, blade, and platform fragments } \\
\text { CAI=3 } \\
\text { [79EK180C] }\end{array}$ & $\begin{array}{l}\text { late Late Devonian-late Early } \\
\text { Mississippian (Famennian- } \\
\text { Osagean). }\end{array}$ & $\begin{array}{l}\text { Indeterminate (too few } \\
\text { conodonts). }\end{array}$ & $\begin{array}{l}\text { Sample from top of interval of shaly limestone. } \\
\text { Po. communis (as its name implies) is an extremely } \\
\text { common conodont throughout its range worldwide, } \\
\text { but is rare in samples of this age from the Red Dog } \\
\text { area. } \\
\text { Fossil loc. 25, Mayfield and others (1990). }\end{array}$ \\
\hline $\begin{array}{l}19 \\
\text { PCA (Amaruk } \\
\text { plate) }\end{array}$ & \begin{tabular}{|l|} 
De Long Mtns. \\
A-3 \\
$68^{\circ} 08^{\prime} 55.7^{\prime \prime /}$ \\
$163^{\circ} 23^{\prime} 9.7^{\prime \prime}$
\end{tabular} & $\begin{array}{l}8 \text { Pa elements Bispathodus utahensis Sandberg and } \\
\text { Gutschick } \\
6 \text { juvenile Pa elements Gnathodus girtyi Hass? } \\
1 \text { Pa element Gnathodus cf. G. pseudosemiglaber } \\
\text { Thompson and Fellows } \\
\text { Hindeodus cristulus (Youngquist and Miller) } \\
10 \text { Pa and } 1 \text { Pb elements } \\
\text { Unassigned elements: } \\
7 \mathrm{M} \text { ( } 2 \text { morphotypes), } 1 \mathrm{Sa}, 3 \mathrm{Sb} \text { ( } 2 \text { morphotypes) } \\
\& 4 \mathrm{Sc} \text { ( } 3 \text { morphotypes) } \\
100 \text { indet. bar, blade, and platform fragments } \\
\text { CAI=3.5 } \\
\text { [8-17-83A; 29235-PC] }\end{array}$ & $\begin{array}{l}\text { middle Late Mississippian } \\
\text { (late Meramecian) on the } \\
\text { basis of the conodonts in } \\
\text { this sample and in 8-17- } \\
\text { 83B (loc. 20) (see } \\
\text { Remarks). }\end{array}$ & $\begin{array}{l}\text { Mixed biofacies; } \\
\text { postmortem mixing of } \\
\text { conodonts from a } \\
\text { range of normal- } \\
\text { marine shelf or } \\
\text { platform } \\
\text { environments. }\end{array}$ & $\begin{array}{l}\text { Sample taken within } 15 \mathrm{~m} \text { of top of Lisburne Group } \\
\text { and } 150 \text { to } 200 \mathrm{~m} \text { stratigraphically above } 8-17-83 \mathrm{~B} \\
\text { (loc. } 20 \text { ). } \\
\text { Medium-dark-gray, medium-light-gray-weathering, } \\
\text { very fine grained, massive dolostone interbedded } \\
\text { with laminated chert. Thin section is nonferroan, } \\
\text { slightly calcitic(?) dolostone; crystals } 20 \text { to } 140 \mu \mathrm{m} \text {. } \\
4.94 \mathrm{~kg} \text { of rock was processed. } \\
\text { Sample collected by A.G. Harris. } \\
\text { Loc. } 8 \text { (fig. } 2 \text { ) of Dumoulin and others (2004). }\end{array}$ \\
\hline
\end{tabular}


Table 4. Conodont samples from deep-water facies of the Lisburne Group in the Picnic Creek allochthon-Continued.

[All faunas identified by A.G. Harris. Structural units determined by L.E. Young. PCA, Picnic Creek allochthon. CAI, conodont color alteration index. Letters in field number refer to collector unless otherwise indicated: AD, J.A. Dumoulin; EK, I. Ellersieck; JS, J.M. Schmidt; and KE, K. Evans. Lithologic data under remarks are field descriptions unless otherwise indicated; thin section observations by J.A. Dumoulin. No., number; indet., indeterminate; loc., locality]

\begin{tabular}{|c|c|c|c|c|c|}
\hline $\begin{array}{l}\text { Locality No., } \\
\text { structural unit }\end{array}$ & $\begin{array}{l}\text { Quadrangle, } \\
\text { latitude n./ } \\
\text { longitude w. }\end{array}$ & $\begin{array}{c}\text { Conodont fauna and CAI } \\
\text { [field No.; USGS collection No.] }\end{array}$ & Age & $\begin{array}{c}\text { Conodont biofacies and } \\
\text { depositional } \\
\text { environment }\end{array}$ & Remarks \\
\hline \begin{tabular}{|l}
20 \\
PCA (Amaruk \\
plate)
\end{tabular} & $\begin{array}{l}\text { De Long Mtns. } \\
\text { A-3 } \\
68^{\circ} 08^{\prime} 49.8^{\prime \prime} / \\
163^{\circ} 23^{\prime} 18.4^{\prime \prime}\end{array}$ & $\begin{array}{l}2 \text { Pa elements Bispathodus utahensis Sandberg and } \\
\text { Gutschick } \\
\text { Cavusgnathus regularis Youngquist and Miller } \\
3 \mathrm{~Pa} \text { and } 1 \mathrm{M} \text { elements [pl. 1, fig. 26] } \\
2 \text { Sb-Sc elements Kladognathus sp. indet. } \\
1 \text { unassigned Sa element } \\
40 \text { indet. bar, blade, and platform fragments } \\
\text { CAI=3.5 } \\
\text { [8-17-83B; 29236-PC] }\end{array}$ & $\begin{array}{l}\text { middle Late Mississippian } \\
\text { (late Meramecian). }\end{array}$ & $\begin{array}{l}\text { Indeterminate (too few } \\
\text { conodonts); species } \\
\text { association indicates } \\
\text { derivation from a } \\
\text { shallow and (or) mid- } \\
\text { shelf or platform } \\
\text { environment. }\end{array}$ & $\begin{array}{l}\text { Sample taken } 150-200 \mathrm{~m} \text { stratigraphically below } 8-17- \\
83 \mathrm{~A} \text { (loc. 19) from sequence of thin- to medium- } \\
\text { bedded dolostone, siliceous dolostone, and blue- } \\
\text { black laminated chert. } \\
\text { Sampled } 8 \text {-inch bed of medium-dark-gray, light-gray- } \\
\text { weathering, laminated, very fine grained dolostone. } \\
\text { Thin section is nonferroan, finely crystalline (20 to } \\
150 \mu \text { m) calcitic dolostone. } \\
5.0 \mathrm{~kg} \text { of rock was processed. } \\
\text { Sample collected by A.G. Harris. } \\
\text { Loc. } 8 \text { (fig. } 2 \text { ) of Dumoulin and others (2004). }\end{array}$ \\
\hline $\begin{array}{l}34 \\
\text { PCA (Wulik } \\
\text { plate) }\end{array}$ & $\begin{array}{l}\text { De Long Mtns. } \\
\text { B-2 } \\
68^{\circ} 21^{\prime} 54^{\prime \prime} / \\
162^{\circ} 50^{\prime} 30^{\prime \prime}\end{array}$ & $\begin{array}{l}\text { Barren } \\
{[\text { KE98-24 (+10 ft)] }}\end{array}$ & & & $\begin{array}{l}\text { Part of a section of Lisburne Group originally } \\
\text { measured and sampled by K.Evans and A. Banet in } \\
1998 \text { and then remeasured and more extensively } \\
\text { sampled by J. Dumoulin and A. Harris in } 1999 \text { and } \\
2000 .^{*} \text {, position within section recalibrated by } \\
\text { Dumoulin and Harris. \#, sample taken by Dumoulin } \\
\text { and Harris. } \\
\text { Sample taken } ~ 10 \text { ft above base of section and base of } \\
\text { Lisburne Group, which overlies Kayak Shale. } \\
\text { Large ( } 1 \text { x } 3 \text { m), light-brownish-gray-weathering } \\
\text { carbonate concretion(?) or slump block in thin- } \\
\text { bedded, silicified, dark-gray noncarbonate } \\
\text { mudstone. Thin section contains abundant } \\
\text { radiolarians in a matrix of darker (muddy?) fine- } \\
\text { grained carbonate (dolomite?). Most radiolarians } \\
\text { replaced by a single calcite crystal, but details of test } \\
\text { structure locally well preserved. Trace detrital quartz } \\
\text { and plagioclase. } \\
6.2 \text { kg of rock was processed. } \\
\text { Loc. } 3 \text { (app. 1) of Dumoulin and others (2004). }\end{array}$ \\
\hline
\end{tabular}


Table 4. Conodont samples from deep-water facies of the Lisburne Group in the Picnic Creek allochthon-Continued.

[All faunas identified by A.G. Harris. Structural units determined by L.E. Young. PCA, Picnic Creek allochthon. CAI, conodont color alteration index. Letters in field number refer to collector unless otherwise indicated: AD, J.A. Dumoulin; EK, I. Ellersieck; JS, J.M. Schmidt; and KE, K. Evans. Lithologic data under remarks are field descriptions unless otherwise indicated; thin section observations by J.A. Dumoulin. No., number; indet., indeterminate; loc., locality]

\begin{tabular}{|c|c|c|c|c|c|}
\hline $\begin{array}{l}\text { Locality No., } \\
\text { structural unit }\end{array}$ & $\begin{array}{l}\text { Quadrangle, } \\
\text { latitude n./ } \\
\text { longitude w. }\end{array}$ & $\begin{array}{c}\text { Conodont fauna and CAI } \\
\text { [field No.; USGS collection No.] }\end{array}$ & Age & $\begin{array}{c}\text { Conodont biofacies and } \\
\text { depositional } \\
\text { environment } \\
\end{array}$ & Remarks \\
\hline \multirow[t]{3}{*}{$\begin{array}{l}34 \\
\text { PCA (Wulik } \\
\text { plate) } \\
\text { [Cont.] }\end{array}$} & \multirow[t]{3}{*}{$\begin{array}{l}\text { De Long Mtns. } \\
\text { B-2 } \\
68^{\circ} 21^{\prime} 54^{\prime \prime /} \\
162^{\circ} 50^{\prime} 30^{\prime \prime}\end{array}$} & $\begin{array}{l}\text { Barren } \\
\text { [KE98-24 (+ } 80 \mathrm{ft})] \#\end{array}$ & & & $\begin{array}{l}\text { Sample taken } \sim 80 \mathrm{ft} \text {. above base of measured section. } \\
\text { Pinkish-gray-weathering, dark-gray, } 30 \text { - to } 100-\mathrm{cm}- \\
\text { thick beds of laminated(?), blocky-weathering, fine- } \\
\text { grained calcareous radiolarite. Thin-section is } \\
\text { dolomitic calcareous radiolarite with lesser } \\
\text { calcareous spicules. } \\
\text { Heavy-mineral concentrate is chiefly composite } \\
\text { phosphatic carbonate rock fragments with minor to } \\
\text { common bioclasts (including phosphatized spicules } \\
\text { and spines, phosphatized steinkerns of ostracodes, } \\
\text { radiolarians, and spines, and phosphatic brachiopod } \\
\text { fragments). Radiolarian spines are abundant in the } \\
<60 \text { mesh heavy-mineral fraction. } \\
11.0 \mathrm{~kg} \text { of rock was processed. }\end{array}$ \\
\hline & & $\begin{array}{l}1 \text { Pa element Bispathodus utahensis Sandberg and } \\
\text { Gutschick } \\
6 \text { indet. bar, blade, and platform fragments } \\
\text { CAI=3 } \\
\text { [KE98-24 (+210 ft); 33641-PC]\# }\end{array}$ & $\begin{array}{l}\text { Early, but not earliest, } \\
\text { Mississippian (late } \\
\text { Kinderhookian to at least } \\
\text { Osagean). }\end{array}$ & $\begin{array}{l}\text { Indeterminate (too few } \\
\text { conodonts). }\end{array}$ & $\begin{array}{l}\text { Sample taken } \sim 210 \mathrm{ft} \text {. above base of measured section. } \\
\text { Medium-dark-gray, fine-grained, partly dolomitized } \\
\text { limestone containing probable small bioclasts. } \\
\text { Collected } 20 \text {-cm-thick relatively chert free bed. Thin } \\
\text { section is bioturbated calcareous spiculite. } \\
12.1 \mathrm{~kg} \text { of rock was processed. }\end{array}$ \\
\hline & & $\begin{array}{l}6 \text { Pa elements Bispathodus utahensis Sandberg and } \\
\text { Gutschick [pl. 1, figs. 21, 22] } \\
37 \text { indet. bar, blade, and platform fragments } \\
\text { CAI }=2 \text { or } 3 \\
\text { [KE98-24 (+308 ft); 33600-PC]* }\end{array}$ & $\begin{array}{l}\text { Early, but not earliest, } \\
\text { Mississippian (late } \\
\text { Kinderhookian-Osagean). }\end{array}$ & $\begin{array}{l}\text { Indeterminate (too few } \\
\text { generically } \\
\text { identifiable } \\
\text { conodonts); likely } \\
\text { postmortem transport } \\
\text { within or from a } \\
\text { bispathodid biofacies. } \\
\text { The conodonts and } \\
\text { associated spicules } \\
\text { and radiolarians } \\
\text { indicate a basinal } \\
\text { depositional setting. }\end{array}$ & $\begin{array}{l}\text { Sample taken } ~ 210 \mathrm{ft} \text { above base of measured section. } \\
\text { Very fine grained lime mudstone lens several meters } \\
\text { thick within black shale. Thin section is finely } \\
\text { interlaminated micrite and noncarbonate mud that } \\
\text { contain } 30-50 \% \text { sponge spicules (most calcareous, } \\
\text { some siliceous, some phosphatized), minor } \\
\text { calcareous and pyritized radiolarians, crinoid } \\
\text { ossicles, and quartz silt and sand. } \\
\text { Heavy-mineral concentrate is chiefly phosphatized } \\
\text { composite grains, phosphatized spicules, radiolarian } \\
\text { steinkerns, and minor pyrite and pyritized- } \\
\text { phosphatized composite grains, spicules, and } \\
\text { radiolarians. } \\
6.6 \text { kg of rock was processed. }\end{array}$ \\
\hline
\end{tabular}


Table 4. Conodont samples from deep-water facies of the Lisburne Group in the Picnic Creek allochthon-Continued.

[All faunas identified by A.G. Harris. Structural units determined by L.E. Young. PCA, Picnic Creek allochthon. CAI, conodont color alteration index. Letters in field number refer to collector unless otherwise indicated: AD, J.A. Dumoulin; EK, I. Ellersieck; JS, J.M. Schmidt; and KE, K. Evans. Lithologic data under remarks are field descriptions unless otherwise indicated; thin section observations by J.A. Dumoulin. No., number; indet., indeterminate; loc., locality]

\begin{tabular}{|c|c|c|c|c|c|}
\hline $\begin{array}{l}\text { Locality No., } \\
\text { structural unit }\end{array}$ & $\begin{array}{l}\text { Quadrangle, } \\
\text { latitude n./ } \\
\text { longitude w. }\end{array}$ & $\begin{array}{c}\text { Conodont fauna and CAI } \\
\text { [field No.; USGS collection No.] }\end{array}$ & Age & $\begin{array}{c}\text { Conodont biofacies and } \\
\text { depositional } \\
\text { environment }\end{array}$ & Remarks \\
\hline \multirow[t]{4}{*}{$\begin{array}{l}34 \\
\text { PCA (Wulik } \\
\text { plate) } \\
\text { [Cont.] }\end{array}$} & \multirow[t]{4}{*}{$\begin{array}{l}\text { De Long Mtns. } \\
\text { B-2 } \\
68^{\circ} 21^{\prime} 54^{\prime \prime} / \\
162^{\circ} 50^{\prime} 30^{\prime \prime}\end{array}$} & $\begin{array}{l}1 \text { indet. bar fragment } \\
\text { CAI=3 } \\
{[\text { KE98-24 (+273 ft); 33642-PC]\# }}\end{array}$ & $\begin{array}{l}\text { Early, but not earliest, } \\
\text { Mississippian (late } \\
\text { Kinderhookian to at least } \\
\text { Osagean). }\end{array}$ & $\begin{array}{l}\text { Indeterminate (too few } \\
\text { conodonts). }\end{array}$ & $\begin{array}{l}\text { Sample taken } \sim 273 \mathrm{ft} \text { above base of measured section. } \\
\text { Collected } 20 \text {-cm-thick bed near top of prominent } 15-\mathrm{ft} \\
\text { outcrop that becomes increasingly carbonate rich } \\
\text { upward. Medium-dark-gray, yellowish-gray- } \\
\text { weathering, fine-grained carbonate with probable } \\
\text { small bioclasts and millimeter-scale parallel } \\
\text { laminae. Thin section is calcareous spiculite with } \\
\text { lesser calcitized radiolarians. } \\
\text { Heavy-mineral concentrate includes rare phosphatized } \\
\text { pelmatozoan ossicles. } \\
8.86 \mathrm{~kg} \text { of rock was processed. } \\
\end{array}$ \\
\hline & & $\begin{array}{l}\text { All conodonts are fragments } \\
\text { Kladognathus } \text { sp. indet. } \\
2 \mathrm{~Pa}-\mathrm{Pb}, 4 \mathrm{M}, 2 \mathrm{Sa} \text {, and } 3 \mathrm{Sb}-\mathrm{Sc} \text { elements } \\
\text { Hindeodus crassidentatus }(\text { Branson and Mehl) } \\
1 \mathrm{~Pb}, 2 \mathrm{M}, 2 \mathrm{Sa} \text {, and } 1 \mathrm{Sc} \text { elements [pl. 2, figs. } 1 \text {, } \\
\text { 2] } \\
92 \text { indet. bar, blade, and platform fragments } \\
\mathrm{CAI}=2.5-3 \\
{[\mathrm{KE} 98-24(+382 \mathrm{ft}) ; 33601-\mathrm{PC}]^{*}}\end{array}$ & $\begin{array}{l}\text { Early, but not earliest } \\
\text { Mississippian (late } \\
\text { Kinderhookian to } \\
\text { Osagean). }\end{array}$ & $\begin{array}{l}\text { Indeterminate (too few } \\
\text { generically } \\
\text { identifiable } \\
\text { conodonts); } \\
\text { postmortem transport } \\
\text { within or from a } \\
\text { shallow-water } \\
\text { depositional setting. }\end{array}$ & $\begin{array}{l}\text { Sample taken } \sim 275 \mathrm{ft} \text { above base of measured section. } \\
\text { Dolomitic(?) limestone beds, } 25 \text { to } 30 \mathrm{~cm} \text { thick. Thin } \\
\text { section is irregularly laminated, subhedral, fine- } \\
\text { grained dolomite(?) with siliceous and calcareous } \\
\text { sponge spicules, rare radiolarians and chert nodules; } \\
\text { some laminae are rich in peloids. } \\
5.0 \mathrm{~kg} \text { of rock was processed. }\end{array}$ \\
\hline & & $\begin{array}{l}2 \text { juvenile Pa elements Gnathodus sp. indet. } \\
2 \text { juvenile Pa elements Vogelgnathus cf. V. } \\
\text { pesaquidi Purnell and von Bitter [pl. 2, figs. 3, 4] } \\
7 \text { indet. bar, blade, and platform fragments } \\
\text { CAI }=2.5-3 \\
{[\text { KE98-24 (+360 ft);33643-PC]\# }}\end{array}$ & $\begin{array}{l}\text { Late Mississippian (early } \\
\text { Meramecian-middle } \\
\text { Chesterian). }\end{array}$ & $\begin{array}{l}\text { Indeterminate (too few } \\
\text { conodonts); size of } \\
\text { conodonts suggests a } \\
\text { postmortem winnow } \\
\text { within or from a slope } \\
\text { or basin depositional } \\
\text { setting. }\end{array}$ & $\begin{array}{l}\text { Sample taken } \sim 360 \mathrm{ft} \text { above base of measured section, } \\
\text { at base of continuous thick carbonate-rich interval. } \\
\text { Medium-light-gray- and light-brownish-gray- } \\
\text { weathering, medium-dark-gray, very fine grained } \\
\text { limestone beds } 2-10 \mathrm{~cm} \text { thick with faint laminae and } \\
\text { chert layers parallel to bedding that make up } 5-15 \% \\
\text { of outcrop. Thin-section is calcareous spiculite with } \\
\text { siliceous nodules and lenses. } \\
\text { Heavy-mineral concentrate is chiefly phosphatic } \\
\text { brachiopod fragments. } \\
10.9 \mathrm{~kg} \text { of rock was processed. } \\
\end{array}$ \\
\hline & & $\begin{array}{l}\text { Barren } \\
{[\text { KE98-24 (+505 ft) }]^{*}}\end{array}$ & & & $\begin{array}{l}\text { Sample taken } ~ 380 \mathrm{ft} \text { above base of measured section. } \\
\text { Very fine grained lime mudstone in contorted beds; } \\
\text { may be a slump block. Thin section is finely } \\
\text { laminated calcareous spiculite with minor calcitized } \\
\text { radiolarians, peloids, and articulated ostracodes. } \\
\text { Heavy-mineral concentrate is chiefly fluorite. } \\
6.2 \mathrm{~kg} \text { of rock was processed. }\end{array}$ \\
\hline
\end{tabular}


Table 4. Conodont samples from deep-water facies of the Lisburne Group in the Picnic Creek allochthon-Continued.

[All faunas identified by A.G. Harris. Structural units determined by L.E. Young. PCA, Picnic Creek allochthon. CAI, conodont color alteration index. Letters in field number refer to collector unless otherwise indicated: AD, J.A. Dumoulin; EK, I. Ellersieck; JS, J.M. Schmidt; and KE, K. Evans. Lithologic data under remarks are field descriptions unless otherwise indicated; thin section observations by J.A. Dumoulin. No., number; indet., indeterminate; loc., locality]

\begin{tabular}{|c|c|c|c|c|c|}
\hline $\begin{array}{l}\text { Locality No., } \\
\text { structural unit }\end{array}$ & $\begin{array}{l}\text { Quadrangle, } \\
\text { latitude n./ } \\
\text { longitude w. }\end{array}$ & $\begin{array}{c}\text { Conodont fauna and CAI } \\
\text { [field No.; USGS collection No.] }\end{array}$ & Age & $\begin{array}{c}\text { Conodont biofacies and } \\
\text { depositional } \\
\text { environment }\end{array}$ & Remarks \\
\hline $\begin{array}{l}34 \\
\text { PCA (Wulik } \\
\text { plate) } \\
\text { [Cont.] }\end{array}$ & $\begin{array}{l}\text { De Long Mtns. } \\
\text { B-2 } \\
68^{\circ} 21^{\prime} 54^{\prime \prime} / \\
162^{\circ} 50^{\prime} 30^{\prime \prime}\end{array}$ & $\begin{array}{l}1 \text { juvenile Pa element fragment Bispathodus } \\
\text { utahensis Sandberg and Gutschick } \\
1 \text { juvenile Pa element Cavusgnathus regularis } \\
\text { Youngquist and Miller [pl. 1, fig. 20] } \\
2 \text { M elements Kladognathus sp. } \\
24 \text { indet. bar, blade, and platform fragments } \\
\text { CAI=3 } \\
\text { [KE98-24 (+590 ft); 33602-PC]* }\end{array}$ & $\begin{array}{l}\text { middle Late Mississippian } \\
\text { (late Meramecian). }\end{array}$ & $\begin{array}{l}\text { Indeterminate (too few } \\
\text { conodonts); } \\
\text { postmortem winnow } \\
\text { into quiet-water } \\
\text { (probably deep-water) } \\
\text { depositional setting. }\end{array}$ & $\begin{array}{l}\text { Sample taken } \sim 440 \mathrm{ft} \text { above base of measured section. } \\
\text { Top of measured section and close to top of Lisburne } \\
\text { Group. Fine-grained limestone. Thin section is } \\
\text { finely laminated calcareous spiculite with minor } \\
\text { calcitized radiolarians, peloids, and articulated } \\
\text { ostracodes. } \\
6.0 \mathrm{~kg} \text { of rock was processed. }\end{array}$ \\
\hline $\begin{array}{l}35 \\
\text { PCA (Wulik } \\
\text { plate) }\end{array}$ & \begin{tabular}{|l|} 
De Long Mtns. \\
B-2 \\
$68^{\circ} 21^{\prime} 56^{\prime \prime} /$ \\
$162^{\circ} 49^{\prime} 54.5^{\prime \prime}$
\end{tabular} & $\begin{array}{l}\text { Conodonts are predominantly incomplete and } \\
\text { somewhat common within a large phosphatized } \\
\text { heavy-mineral concentrate. } \\
\text { early and middle Osagean conodonts: } \\
\text { 7 Pa elements Gnathodus cuneiformis Mehl and } \\
\text { Thomas [pl. 1, fig. 24] } \\
\text { Osagean-early Chesterian conodonts: } \\
1 \text { Pa element Gnathodus semiglaber Bischoff? } \\
\text { late Osagean-early Chesterian conodonts: } \\
\text { 36 Pa elements (mostly fragments) Gnathodus } \\
\text { texanus Roundy [pl. 1, fig. 25] } \\
\text { 18 Idioprioniodus sp. indet. fragments } \\
\text { early Chesterian conodonts: } \\
\text { 2 Pa elements Rhachistognathus prolixus } \\
\text { Baesemann and Lane [pl. 1, fig. 23] } \\
\text { Unassigned elements: } \\
\text { 11 Pb elements (mostly fragments) of Osagean- } \\
\text { Meramecian morphotype } \\
\text { 178 indet. bar, blade, and platform fragments } \\
\text { CAI=2.5 } \\
\text { [99AD19D; 33481-PC] }\end{array}$ & $\begin{array}{l}\text { No older than middle Late } \\
\text { Mississippian (early } \\
\text { Chesterian): mixture of } \\
\text { early-middle Osagean, late } \\
\text { Osagean-early Chesterian } \\
\text { conodonts. }\end{array}$ & $\begin{array}{l}\text { Indeterminate; lag } \\
\text { concentrate } \\
\text { containing a mixture } \\
\text { of largely outer shelf } \\
\text { to off shelf conodonts } \\
\text { of mixed Osagean to } \\
\text { at least early } \\
\text { Chesterian age. }\end{array}$ & $\begin{array}{l}\text { Samples stratigraphically highest carbonate bed in } \\
\text { Lisburne Group here; underlies grassy swale with } \\
\text { black silicified mudstone float (uppermost Lisburne } \\
\text { Group?) and then medium-gray-green radiolarian } \\
\text { chert (Etivluk Group). See table } 14 \text { for radiolarian } \\
\text { samples from the Etivluk at this locality. 99AD19D } \\
\text { is approximately equivalent to highest part of } \\
\text { measured section KE98-24 (loc. 34), which is } 0.3 \\
\text { miles to southwest. } \\
\text { Very fetid limestone (crinoidal grainstone) with 3- to } \\
\text { 4-mm-long black mudstone clasts; forms 0.5-m- } \\
\text { thick irregular outcrop with local black chert } \\
\text { nodules. Thin section is crinoidal grainstone with } \\
\text { bryozoan fragments and brachiopod(?) spines; many } \\
\text { bioclasts replaced (totally or in part) by phosphate. } \\
\text { Heavy-mineral concentrate is chiefly phosphatized } \\
\text { rock fragments (some ferruginous), phosphatized } \\
\text { bioclasts (including pelmatozoan fragments and } \\
\text { ostracode, gastropod, and pelecypod steinkerns) and } \\
\text { phosphatic brachiopod fragments. } \\
8.6 \text { kg of rock was processed. }\end{array}$ \\
\hline
\end{tabular}


Table 4. Conodont samples from deep-water facies of the Lisburne Group in the Picnic Creek allochthon-Continued.

[All faunas identified by A.G. Harris. Structural units determined by L.E. Young. PCA, Picnic Creek allochthon. CAI, conodont color alteration index. Letters in field number refer to collector unless otherwise indicated: AD, J.A. Dumoulin; EK, I. Ellersieck; JS, J.M. Schmidt; and KE, K. Evans. Lithologic data under remarks are field descriptions unless otherwise indicated; thin section observations by J.A. Dumoulin. No., number; indet., indeterminate; loc., locality]

\begin{tabular}{|c|c|c|c|c|c|}
\hline $\begin{array}{l}\text { Locality No., } \\
\text { structural unit }\end{array}$ & $\begin{array}{l}\text { Quadrangle, } \\
\text { latitude n./ } \\
\text { longitude w. }\end{array}$ & $\begin{array}{c}\text { Conodont fauna and CAI } \\
\text { [field No.; USGS collection No.] }\end{array}$ & Age & \begin{tabular}{|c|}
$\begin{array}{c}\text { Conodont biofacies and } \\
\text { depositional } \\
\text { environment }\end{array}$ \\
\end{tabular} & Remarks \\
\hline $\begin{array}{l}37 \\
\text { PCA (Wulik } \\
\text { plate) }\end{array}$ & $\begin{array}{l}\text { De Long Mtns. } \\
\text { B-2 } \\
68^{\circ} 21^{\prime} 23^{\prime \prime} / \\
162^{\circ} 56^{\prime} 33.7^{\prime \prime}\end{array}$ & $\begin{array}{l}2 \text { Pa elements Gnathodus cuneiformis Mehl and } \\
\text { Thomas } \\
1 \text { Pa element Gnathodus sp. indet. } \\
1 \text { Pa element Eotaphrus burlingtonensis Pierce and } \\
\text { Langenheim [pl. 2, figs. 7, 8] } \\
1 \text { Pa element Polygnathus communis Branson and } \\
\text { Mehl [pl. 2, figs. 9, 10] } \\
1 \text { S element bar fragment Scaliognathus anchoralis } \\
\text { Branson and Mehl } \\
60 \text { indet. bar, blade, and platform fragments } \\
\text { CAI=2.5-3 } \\
\text { [79EK184B; 27554-PC] }\end{array}$ & $\begin{array}{l}\text { middle late Early } \\
\text { Mississippian (middle } \\
\text { Osagean; Sc. anchoralis- } \\
\text { Do. latus Zone). }\end{array}$ & $\begin{array}{l}\text { Polygnathid biofacies; } \\
\text { postmortem transport } \\
\text { within or from a } \\
\text { shelfal depositional } \\
\text { setting. }\end{array}$ & $\begin{array}{l}\text { Crinoidal limestone near contact with noncalcareous } \\
\text { cherts and shales. } \\
8.2 \mathrm{~kg} \text { of rock was processed. } \\
\text { Fossil loc. 13, Ellersieck and others (1990). }\end{array}$ \\
\hline $\begin{array}{l}38 \\
\text { PCA (Wulik } \\
\text { plate) }\end{array}$ & $\begin{array}{l}\text { De Long Mtns. } \\
\text { B-2 } \\
68^{\circ} 20^{\prime} 43.8^{\prime \prime} / \\
162^{\circ} 56^{\prime} 41.2^{\prime \prime}\end{array}$ & $\begin{array}{l}2 \text { "Hindeodella segaformis" Bischoff s.f. element } \\
\text { fragments } \\
4 \text { indet. bar and blade fragments } \\
\text { Conodonts are partly to substantially covered with } \\
\text { adventitious organic matter so that CAI is } \\
\text { approximate but not greater than } 3 \text {. } \\
\text { [98AD11D; 33417-PC] }\end{array}$ & $\begin{array}{l}\text { middle late Early } \\
\text { Mississippian (middle } \\
\text { Osagean; Sc. anchoralis-D, } \\
\text { latus Zone). }\end{array}$ & $\begin{array}{l}\text { Indeterminate (too few } \\
\text { conodonts); } \\
\text { conodonts present } \\
\text { indicate relatively } \\
\text { deep-water } \\
\text { depositional setting, } \\
\text { probably a } \\
\text { postmortem winnow. }\end{array}$ & $\begin{array}{l}\text { 20-m-thick section of thin-bedded to laminated, } \\
\text { medium-gray, very fine grained limestone with } \\
\text { black to brown shale partings and thin chert } \\
\text { interbeds. Calcareous layers here are probable } \\
\text { turbidites. Thin section is 1- to 4-mm-thick layers of } \\
\text { calcisilt and fine-grained calcisand alternating with } \\
0.5 \text { - to } 1 \text {-mm-thick layers of brown lime mud; } \\
\text { scattered dolomite rhombs (60-100 } \mu \text { m) especially in } \\
\text { muddy layers. Rare identifiable bioclasts mostly } \\
\text { calcareous and lesser siliceous spicules. } \\
\text { Heavy-mineral concentrate is chiefly angular to } \\
\text { subrounded green to blue-green mineral (glauconite } \\
\text { or zeolite?). } \\
9.5 \text { kg of rock was processed. }\end{array}$ \\
\hline $\begin{array}{l}39 \\
\text { PCA (Amaruk } \\
\text { plate) }\end{array}$ & $\begin{array}{l}\text { De Long Mtns. } \\
\text { B-2 } \\
68^{\circ} 17^{\prime} 28.5^{\prime \prime} / \\
163^{\circ} 11^{\prime} 52.9^{\prime \prime}\end{array}$ & $\begin{array}{l}16 \text { Pa elements (incomplete or fragments) } \\
\text { Bispathodus stabilis (Branson and Mehl) or } \\
\text { Bispathodus utahensis Sandberg and Gutschick } \\
\text { Kladognathus sp. indet. } \\
2 \mathrm{M}, 1 \mathrm{Sa} \text {, and } 2 \mathrm{Sb}-\mathrm{Sc} \text { element fragments } \\
1 \text { unassigned } \mathrm{M} \text { element fragment } \\
61 \text { indet. bar, blade, and platform fragments } \\
\text { CAI=2.5-3 } \\
\text { [KE98-15B; 33502-PC] }\end{array}$ & $\begin{array}{l}\text { Early, but not earliest, } \\
\text { through early Late } \\
\text { Mississippian (middle } \\
\text { Kinderhookian- } \\
\text { Meramecian) }\end{array}$ & $\begin{array}{l}\text { Postmortem transport } \\
\text { from or within the } \\
\text { bispathodid biofacies; } \\
\text { depositional setting } \\
\text { difficult to refine as } \\
\text { both single-row } \\
\text { bispathodids and } \\
\text { kladognathids were } \\
\text { likely surface } \\
\text { swimmers. }\end{array}$ & $\begin{array}{l}\text { Light-gray-weathering, medium-light-gray, fine- } \\
\text { grained crinoidal dolostone, laminated and } \\
\text { interbedded with gray and tan chert. Outcrop } \\
\text { features suggest a distal ramp setting. Thin section is } \\
\text { dolostone with relict crinoid fragments. } \\
\text { Heavy-mineral concentrate includes very pale purple } \\
\text { fluorite. } \\
3.3 \mathrm{~kg} \text { of rock was processed. }\end{array}$ \\
\hline
\end{tabular}


Table 4. Conodont samples from deep-water facies of the Lisburne Group in the Picnic Creek allochthon-Continued.

[All faunas identified by A.G. Harris. Structural units determined by L.E. Young. PCA, Picnic Creek allochthon. CAI, conodont color alteration index. Letters in field number refer to collector unless otherwise indicated: AD, J.A. Dumoulin; EK, I. Ellersieck; JS, J.M. Schmidt; and KE, K. Evans. Lithologic data under remarks are field descriptions unless otherwise indicated; thin section observations by J.A. Dumoulin. No., number; indet., indeterminate; loc., locality]

\begin{tabular}{|c|c|c|c|c|c|}
\hline $\begin{array}{l}\text { Locality No., } \\
\text { structural unit }\end{array}$ & $\begin{array}{l}\text { Quadrangle, } \\
\text { latitude } \mathrm{n} . / \\
\text { longitude w. }\end{array}$ & $\begin{array}{c}\text { Conodont fauna and CAI } \\
\text { [field No.; USGS collection No.] }\end{array}$ & Age & $\begin{array}{c}\text { Conodont biofacies and } \\
\text { depositional } \\
\text { environment }\end{array}$ & Remarks \\
\hline $\begin{array}{l}59 \\
\text { PCA (Amaruk } \\
\text { plate) }\end{array}$ & $\begin{array}{l}\text { De Long Mtns. } \\
\text { A-2 } \\
68^{\circ} 11^{\prime} 24^{\prime \prime} / \\
163^{\circ} 8^{\prime} 13.1^{\prime \prime}\end{array}$ & $\begin{array}{l}42 \text { juvenile and subadult Pa elements Bispathodus } \\
\text { stabilis (Branson and Mehl) or Bi. utahensis } \\
\text { Sandberg and Gutschick } \\
\text { Kladognathus sp. } \\
18 \mathrm{M}, 9 \mathrm{Sa} \text {, and } 36 \mathrm{Sb}-\mathrm{Sc} \text { elements } \\
\text { Unassigned elements: } \\
5 \mathrm{~Pb} \text { (3 morphotypes), } 7 \mathrm{M} \text { (3 morphotypes), } 1 \\
\mathrm{Sb} \text {, and } 1 \mathrm{Sc} \\
420 \text { indet. bar, blade, and platform fragments } \\
\text { CAI=2.5-3 } \\
\text { [98JS8G2; 33612-PC] }\end{array}$ & $\begin{array}{l}\text { Early, but not earliest, to early } \\
\text { Late Mississippian (middle } \\
\text { Kinderhookian- } \\
\text { Meramecian). }\end{array}$ & $\begin{array}{l}\text { Postmortem transport } \\
\text { within or from the } \\
\text { bispathodid- } \\
\text { kladognathid } \\
\text { biofacies; deep-water } \\
\text { depositional setting. }\end{array}$ & $\begin{array}{l}\text { Fine-grained, sucrosic, locally spiculitic, buff- to tan- } \\
\text { weathering dolostone interbedded with chert. } \\
\text { Dolostone in graded, locally contorted beds } 1 \text { to } 2 \\
\text { cm thick that are probable turbidites. Thin section is } \\
\text { fine-grained euhedral dolomite mosaic with trace } \\
\text { detrital quartz silt and sand and local phosphatic(?) } \\
\text { laminae. } \\
\text { Heavy-mineral concentrate consists chiefly of } \\
\text { phosphatized composite grains. } \\
5.8 \text { kg of rock was processed. }\end{array}$ \\
\hline $\begin{array}{l}117 \\
\text { PCA (Wulik } \\
\text { plate) }\end{array}$ & $\begin{array}{l}\text { Misheguk Mtn. } \\
\text { B-5 } \\
68^{\circ} 21^{\prime} 54^{\prime \prime} / \\
161^{\circ} 46^{\prime} 15^{\prime \prime}\end{array}$ & $\begin{array}{l}\text { All conodonts are robust fragments reflecting the } \\
\text { high-energy derivation of their host rock. } \\
6 \text { Idioprioniodus sp. indet. robust fragments } \\
10 \text { incomplete Pa elements Pseudopolygnathus } \\
\text { oxypageus Lane, Sandberg, and Ziegler } \\
36 \text { Pa elements Pseudopolygnathus spp. indet. } \\
1 \text { unassigned M elements } \\
31 \text { indet. bar, blade, and platform fragments } \\
\text { CAI= 2.5 } \\
{[00 \mathrm{AD} 25 \mathrm{~A} ; 33636-\mathrm{PC}]}\end{array}$ & $\begin{array}{l}\text { late Early Mississippian (late } \\
\text { early-middle Osagean; } \\
\text { Upper G. typicus Zone } \\
\text { through lower } 2 / 3 \text { of } \\
\text { succeeding Sc. anchoralis- } \\
\text { Do. latus Zone). }\end{array}$ & $\begin{array}{l}\text { Postmortem transport } \\
\text { from or within the } \\
\text { pseudopolygnathid } \\
\text { biofacies; postmortem } \\
\text { transport within or } \\
\text { from an outer shelf or } \\
\text { slope depositional } \\
\text { setting. }\end{array}$ & $\begin{array}{l}\text { Medium-gray- and pale yellow-brown-weathering, } \\
\text { medium-dark-gray, pelmatozoan grainstone from } \\
0.5-3 \mathrm{~cm} \text { thick beds above and below thicker chert } \\
\text { layers. Chert beds contain abundant silicified } \\
\text { pelmatozoan ossicles. Thin-section is a diverse } \\
\text { skeletal supportstone; bioclasts include crinoids, } \\
\text { brachiopods, ostracodes, and foraminifers. } \\
\text { Heavy-mineral concentrate is chiefly dolomitized } \\
\text { bioclasts (predominantly pelmatozoan ossicles and } \\
\text { rare phosphatized fenestrate bryozoan fragments) } \\
\text { and minor fluorite. } \\
13.1 \text { kg of rock was processed. } \\
\text { Loc. } 4 \text { (app. 1) of Dumoulin and others (2004). }\end{array}$ \\
\hline
\end{tabular}


Table 4. Conodont samples from deep-water facies of the Lisburne Group in the Picnic Creek allochthon-Continued.

[All faunas identified by A.G. Harris. Structural units determined by L.E. Young. PCA, Picnic Creek allochthon. CAI, conodont color alteration index. Letters in field number refer to collector unless otherwise indicated: AD, J.A. Dumoulin; EK, I. Ellersieck; JS, J.M. Schmidt; and KE, K. Evans. Lithologic data under remarks are field descriptions unless otherwise indicated; thin section observations by J.A. Dumoulin. No., number; indet., indeterminate; loc., locality]

\begin{tabular}{|c|c|c|c|c|c|}
\hline $\begin{array}{l}\text { Locality No., } \\
\text { structural unit }\end{array}$ & $\begin{array}{l}\text { Quadrangle, } \\
\text { latitude } \mathrm{n} . / \\
\text { longitude w. }\end{array}$ & $\begin{array}{c}\text { Conodont fauna and CAI } \\
\text { [field No.; USGS collection No.] }\end{array}$ & Age & \begin{tabular}{|c|} 
Conodont biofacies and \\
depositional \\
environment
\end{tabular} & Remarks \\
\hline $\begin{array}{l}117 \\
\text { PCA (Wulik } \\
\text { plate) } \\
\text { [Cont.] }\end{array}$ & $\begin{array}{l}\text { Misheguk Mtn. } \\
\text { B-5 } \\
68^{\circ} 21^{\prime} 54^{\prime \prime} / \\
161^{\circ} 46^{\prime} 15^{\prime \prime}\end{array}$ & $\begin{array}{l}5 \text { Pa element fragments Bispathodus utahensis } \\
\text { Sandberg and Gutschick? } \\
\text { Kladognathus tenuis } \\
3 \mathrm{Sa} \text {, } 5 \mathrm{Sb}-\mathrm{Sc} \\
3 \text { juvenile Pa elements Polygnathus communis } \\
\text { Branson and Mehl } \\
\text { 1Sc and } 35 \mathrm{~S} \text { element bar fragments (short and } \\
\text { long) Scaliognathus anchoralis Branson and } \\
\text { Mehl [pl. 2, figs. 5, 6] } \\
1 \text { juvenile Pa element Synclydognathus geminus } \\
\text { (Hinde) } \\
\text { Unassigned elements: } \\
7 \mathrm{~Pb} \text { (5 morphotypes), } 7 \mathrm{M} \text { (5 morphotypes), } 3 \\
\text { Sa, and } 2 \mathrm{Sc} \text { (2 morphotypes) } \\
145 \text { indet. bar, blade, and platform fragments } \\
\text { CAI=2 } \\
\text { [00AD25F; 33637-PC] }\end{array}$ & $\begin{array}{l}\text { middle late Early } \\
\text { Mississippian (middle } \\
\text { Osagean; Sc. anchoralis- } \\
\text { Do. latus Zone). }\end{array}$ & $\begin{array}{l}\text { Post-mortem transport } \\
\quad \text { (likely a winnow) } \\
\text { from a mix of outer } \\
\text { shelf and slope } \\
\text { environments. }\end{array}$ & $\begin{array}{l}\text { Float block on talus slope at same general locale as } \\
\text { 00AD25A. } \\
\text { Block } 40 \text { x } 10 \mathrm{~cm} \text { of light-gray-weathering, pinkish- } \\
\text { medium-dark-gray, fine-grained limestone and black } \\
\text { chert in 2-cm alternations. Limestone partly } \\
\text { silicified. Thin-section is calcareous spiculite with } \\
\text { rare radiolarians; some spicules pyritized. } \\
\text { Heavy-mineral concentrate includes composite } \\
\text { phosphatic, very fine grained siliciclastic rock } \\
\text { fragments and rare sponge spicules. } \\
7.7 \mathrm{~kg} \text { of rock was processed. }\end{array}$ \\
\hline
\end{tabular}


Table 5. Conodont samples from other deep-water strata of the Lisburne Group

[All faunas identified by A.G. Harris. Structural units determined by L.E. Young. EMA, Endicott Mountains allochthon; IRA, Ipnavik River allochthon. CAI, conodont color alteration index. Letters in field number refer to collector: AD, J.A. Dumoulin; and AK, J.S. Kelley. Lithologic data under remarks are field descriptions unless otherwise indicated; thin section observations by J.A. Dumoulin. No., number; indet., indeterminate; loc., locality]

\begin{tabular}{|c|c|c|c|c|c|}
\hline $\begin{array}{l}\text { Locality No., } \\
\text { structural unit }\end{array}$ & $\begin{array}{l}\text { Quadrangle, } \\
\text { latitude n./ } \\
\text { longitude w. }\end{array}$ & $\begin{array}{c}\text { Conodont fauna and CAI } \\
\text { [field No.; USGS collection No.] }\end{array}$ & Age & $\begin{array}{l}\text { Conodont biofacies and } \\
\text { depositional environment }\end{array}$ & Remarks \\
\hline $\begin{array}{l}85 \\
\text { EMA } \\
\text { (Wolverine } \\
\text { Creek plate; } \\
\text { Rok window) }\end{array}$ & $\begin{array}{l}\text { De Long Mtns. } \\
\text { A-2 } \\
68^{\circ} 01^{\prime} 31^{\prime \prime} / \\
162^{\circ} 48^{\prime} 12^{\prime \prime}\end{array}$ & $\begin{array}{l}1 \text { deformed incomplete Pa element Bispathodus } \\
\text { stabilis (Branson and Mehl) or Bi. utahensis } \\
\text { Sandberg and Gutschick } \\
1 \text { unassigned M element } \\
1 \text { indet. blade or platform fragment } \\
\text { CAI=3 } \\
\text { [97AK1A; 33392-PC] }\end{array}$ & $\begin{array}{l}\text { Early Mississippian } \\
\text { (Kinderhookian-Osagean). }\end{array}$ & $\begin{array}{l}\text { Indeterminate (too few } \\
\text { conodonts). }\end{array}$ & $\begin{array}{l}\text { Sample from section of unnamed deep-water facies } \\
\text { underlying the Kogruk Formation. } \\
\text { Sample from } \sim 5 \mathrm{~m} \text { of dark-gray to black chert and } \\
\text { cherty carbonate in nodular beds } 1 \text { to } 5 \mathrm{~cm} \text { thick } \\
\text { that may be partly bioturbated; overlies } \sim 7 \mathrm{~m} \text { of } \\
\text { dark shale and mudstone. Thin section is dolomite } \\
\text { rhombs and rhombohedral clusters } 60 \text { to } 500 \mu \mathrm{m} \\
\text { in diameter in a black organic-rich matrix with a } \\
1-\mathrm{cm} \text {-thick lens of brown spiculitic chert. } \\
7.0 \mathrm{~kg} \text { of rock was processed. }\end{array}$ \\
\hline $\begin{array}{l}89 \\
\text { EMA } \\
\text { (Wolverine } \\
\text { Creek plate; } \\
\text { Rok window) }\end{array}$ & $\begin{array}{l}\text { De Long Mtns. } \\
\text { A-2 } \\
68^{\circ} 00^{\prime} 25^{\prime \prime} / \\
162^{\circ} 48^{\prime} 22.6^{\prime \prime}\end{array}$ & $\begin{array}{l}2 \text { Pa elements Cavusgnathus unicornis Youngquist } \\
\text { and Miller } \\
4 \mathrm{~Pa} \text { elements Gnathodus sp. indet. } \\
1 \mathrm{Sc} \text { element Kladognathus tenuis } \\
\text { Unassigned elements: } \\
3 \mathrm{~Pb}(2 \text { morphotypes) and } 1 \mathrm{Sc} \\
48 \text { indet. bar, blade and platform fragments } \\
\text { CAI=2 or } 3 \text { (nearly all conodonts covered with or } \\
\text { invaded by organic material) } \\
\text { [98AD13A; 33418-PC] }\end{array}$ & $\begin{array}{l}\text { middle-late Late } \\
\text { Mississippian (late } \\
\text { Meramecian-Chesterian). }\end{array}$ & \begin{tabular}{|l|} 
Indeterminate (too few \\
conodonts). The \\
cavusgnathids are very \\
large fragments compared \\
to the rest of the \\
conodont fauna and \\
indicate postmortem \\
transport from a shallow- \\
water environment; the \\
relatively large number of \\
indeterminate fragments \\
also indicates postmortem \\
transport. Two of the \\
gnathodids are complete, \\
suggesting they lived \\
nearby in a middle shelf \\
or deeper water \\
depositional setting. \\
\end{tabular} & $\begin{array}{l}\text { Sample from section of unnamed deep-water facies } \\
\text { depositionally underlying the Kogruk Formation. } \\
\text { Sample from } 0.5 \mathrm{~m} \text { of dark-gray to black, light- } \\
\text { gray-weathering, very fine grained limestone in } 1 \\
\text { to } 4 \text {-cm-thick beds, above } 4 \mathrm{~m} \text { of thinner bedded, } \\
\text { beige, shaly limestone and overlain by brownish } \\
\text { black shale and mudstone. Thin section is very } \\
\text { fine-grained skeletal packstone made up chiefly of } \\
\text { calcareous sponge spicules ( } 20 \times 400 \mu \mathrm{m} \text { or less) } \\
\text { and lesser calcitized radiolarians (test structure } \\
\text { locally well-preserved). } \\
9.7 \mathrm{~kg} \text { of rock was processed. } \\
\text { Loc. } 21 \text { (fig. 2) of Dumoulin and others (2004). }\end{array}$ \\
\hline $\begin{array}{l}120 \\
\text { IRA (Nachralik } \\
\text { Pass plate) }\end{array}$ & $\begin{array}{l}\text { Misheguk Mtn. } \\
\text { B-4 } \\
68^{\circ} 27^{\prime} 47^{\prime \prime} / \\
161^{\circ} 20^{\prime} 7.8^{\prime \prime}\end{array}$ & $\begin{array}{l}\text { Bispathodus utahensis Sandberg and Gutschick } \\
27 \mathrm{~Pa} \text { (mostly fragments) and } 3 \mathrm{~Pb} \text { elements } \\
1 \text { incomplete P element Geniculatus sp. [fig. } 2 \text {, } \\
\text { figs. } 11,12] \\
1 \text { bar fragment Scaliognathus anchoralis Branson } \\
\text { and Mehl } \\
\text { Unassigned elements: } \\
5 \mathrm{~Pb}(4 \text { morphotypes), } 2 \mathrm{M} \text { ( } 2 \text { morphotypes), } 1 \mathrm{Sb} \text {, } \\
\text { and } 5 \mathrm{Sc}(3 \text { morphotypes) } \\
192 \text { indet. bar, blade, and platform fragments } \\
\text { CAI=2.5-3 } \\
\text { [00AD26A; 33638-PC] }\end{array}$ & $\begin{array}{l}\text { middle late Early } \\
\text { Mississippian (middle } \\
\text { Osagean; Sc. anchoralis- } \\
\text { Do. latus Zone). }\end{array}$ & $\begin{array}{l}\text { Postmortem transport } \\
\text { within or from the } \\
\text { bispathodid biofacies; } \\
\text { transport from a shelf and } \\
\text { (or) slope depositional } \\
\text { setting. }\end{array}$ & $\begin{array}{l}\text { Sample from very fine grained, light-gray- } \\
\text { weathering, pinkish medium-gray dolostone layer, } \\
\sim 0.5 \text { to } 1 \mathrm{~m} \text { thick, that forms notable recessive } \\
\text { interval in outcrop of black chert. Dolostone beds } \\
\text { are } 1 \text { to } 6 \mathrm{~cm} \text { thick and parallel- and cross- } \\
\text { laminated. Thin section is dolomitic calcareous } \\
\text { radiolarite. } \\
\text { Heavy-mineral concentrate includes minor } \\
\text { dolomitized bioclasts. } \\
9.0 \mathrm{~kg} \text { of rock was processed. } \\
\text { Loc. } 5 \text { (app. 1) of Dumoulin and others (2004). }\end{array}$ \\
\hline
\end{tabular}


Table 6. Conodont samples from the Kogruk Formation

[All faunas identified by A.G. Harris. Structural units determined by L.E. Young. EMA, Endicott Mountains allochthon; KRA, Kelly River allochthon. CAI, conodont color alteration index. Letters in field number refer to collector: AD, J.A. Dumoulin; AK, J.S. Kelley; AKD, K.D. Kelley; JS, J.M. Schmidt; KE, K. Evans; KS, K.W. Sherwood; MD, C.F. Mayfield; and TR, I.L. Tailleur. DDH, diamond drill hole. Interval thicknesses in drill holes are drilled thicknesses and have not been corrected for Te field descriptions unless otherwise indicated; thin section observations by J.A. Dumoulin. No., number; indet, indeterminate; loc., locality]

\begin{tabular}{|c|c|c|c|c|c|}
\hline $\begin{array}{l}\text { Locality No., } \\
\text { structural unit }\end{array}$ & $\begin{array}{l}\text { Quadrangle, } \\
\text { latitude } \mathrm{n} . / \\
\text { longitude w. }\end{array}$ & $\begin{array}{c}\text { Conodont fauna and CAI } \\
\text { [field No.; USGS collection No.] }\end{array}$ & Age & \begin{tabular}{|c|}
$\begin{array}{c}\text { Conodont biofacies and } \\
\text { depositional } \\
\text { environment }\end{array}$ \\
\end{tabular} & Remarks \\
\hline $\begin{array}{l}2 \\
\text { KRA (Chimney } \\
\text { plate) }\end{array}$ & $\begin{array}{l}\text { De Long Mtns. } \\
\text { A-4 } \\
68^{\circ} 11^{\prime} 14.3^{\prime \prime /} \\
163^{\circ} 56^{\prime} 6.5^{\prime \prime}\end{array}$ & $\begin{array}{l}\text { Virtually all specimens are severely broken or } \\
\text { incomplete. } \\
14 \text { Pa element fragments Bispathodus stabilis } \\
\text { (Branson and Mehl) or Bi. utahensis Sandberg } \\
\text { and Gutschick } \\
\text { Kladognathus sp. } \\
2 \text { Pa-Pb, } 6 \mathrm{M}, 1 \mathrm{Sa} \text {, and } 11 \text { Sb-Sc elements } \\
\text { Synclydognathus geminus (Hinde) } \\
3 \text { Pa and } 9 \mathrm{M} \& \text { S elements } \\
1 \text { unassigned ozarkodinid Sc element } \\
204 \text { indet. bar, blade, and platform fragments } \\
\text { CAI=2 } \\
\text { [99AD26AA; 33482-PC] }\end{array}$ & $\begin{array}{l}\text { Early-middle Late } \\
\text { Mississippian (middle } \\
\text { Kinderhookian-early } \\
\text { Chesterian, probably no } \\
\text { younger than Meramecian). }\end{array}$ & \begin{tabular}{|l|} 
Postmortem transport \\
within or from the \\
kladognathid- \\
bispathodid biofacies; \\
species association \\
and taphonomy \\
indicates a relatively \\
high-energy shallow- \\
water depositional \\
environment.
\end{tabular} & $\begin{array}{l}\text { Sample near base of cherty limestone member of } \\
\text { Kogruk Formation of Mayfield and others (1990) } \\
\text { from 0.5-m-thick interval with no chert bounded by } \\
\text { irregular white chert bands; outcrop contains local } \\
\text { silicified bryozoans and horn corals. } \\
\text { Massive, very light gray-weathering, pinkish-gray, } \\
\text { slightly fetid, sugary calcareous dolostone with } \\
\text { abundant crinoid ossicles. Thin section is partly } \\
\text { dolomitized crinoidal supportstone. } \\
\text { Heavy-mineral concentrate includes phosphatized } \\
\text { bioclasts (bryozoans) and phosphatic bioclasts } \\
\text { (phosphatic brachiopod fragments and ichthyoliths). } \\
9.5 \text { kg of rock was processed. }\end{array}$ \\
\hline $\begin{array}{l}3 \\
\text { Olistolith, } \\
\text { possibly } \\
\text { derived from } \\
\text { KRA }\end{array}$ & $\begin{array}{l}\text { De Long Mtns. } \\
\text { A-4 } \\
68^{\circ} 06^{\prime} 18^{\prime \prime} / \\
164^{\circ} 06^{\prime} 24^{\prime \prime}\end{array}$ & $\begin{array}{l}1 \text { subadult Pa element Gnathodus sp. of late } \\
\text { Osagean-early Chesterian morphotype } \\
1 \text { Sb-Sc element fragment Kladognathus } \text { sp. indet. } \\
3 \text { indet. bar, blade, and platform fragments } \\
\text { CAI=1.5-2 } \\
\text { [79MD180;27567-PC] }\end{array}$ & \begin{tabular}{|l} 
latest Early-middle Late \\
Mississippian (late \\
Osagean-early Chesterian).
\end{tabular} & $\begin{array}{l}\text { Indeterminate (too few } \\
\text { conodonts). } \\
\text { Conodonts indicate } \\
\text { normal-marine } \\
\text { shallow-shelf to slope } \\
\text { environment. }\end{array}$ & $\begin{array}{l}\text { Limestone. } \\
\text { Fossil loc. 32, Mayfield and others (1990). }\end{array}$ \\
\hline $\begin{array}{l}5 \\
\text { KRA (Wulik } \\
\text { Peaks plate) }\end{array}$ & $\begin{array}{l}\text { De Long Mtns. } \\
\text { B-3 } \\
68^{\circ} 17^{\prime} 37^{\prime \prime} / \\
163^{\circ} 22^{\prime} 04^{\prime \prime}\end{array}$ & $\begin{array}{l}7 \text { indet. bar, blade, and platform fragments } \\
\text { CAI difficult to determine as all conodonts are } \\
\text { worn fragments; }>2 \text { to } 3.5-4 \text {. } \\
\text { [00AD4B; 33625-PC] }\end{array}$ & $\begin{array}{l}\text { Mississippian on the basis of } \\
\text { stratigraphic position and } \\
\text { stratigraphic unit. }\end{array}$ & $\begin{array}{l}\text { Indeterminate; no } \\
\text { generically } \\
\text { identifiable } \\
\text { conodonts. }\end{array}$ & $\begin{array}{l}\text { Near top of Kogruk Formation here; sample from } \\
\text { highest carbonate rock overlain by } \sim 3 \text { m of recessive } \\
\text { black shale that contains radiolarian-bearing } \\
\text { phosphate nodules } 3 \text { to } 4 \mathrm{~cm} \text { in diameter. Etivluk } \\
\text { Group chert overlies Kogruk; see table } 14 \text { for } \\
\text { Etivluk radiolarian faunas from this locality. } \\
\text { Light-gray-weathering, medium- to medium-light- } \\
\text { gray, thick- to massive-bedded limestone with } 10- \\
15 \% \text { chert; grainstone/packstone with large bioclasts } \\
\text { including pelmatozoan ossicles and solitary corals. } \\
\text { Thin-section is pelmatozoan-bryozoan packstone } \\
\text { with ostracodes, brachiopod fragments, calcareous } \\
\text { sponge spicules, and a trace of quartz silt; silica } \\
\text { partly replaces some bioclasts. Fabric is packed to } \\
\text { overpacked with some sutured contacts. } \\
\text { Heavy-mineral concentrate includes phosphatized rock } \\
\text { fragments and bioclasts and minor ichthyoliths. } \\
10.55 \mathrm{~kg} \text { of rock was processed. } \\
\text { Loc. } 1 \text { (fig. 2) of Dumoulin and others (2004). }\end{array}$ \\
\hline
\end{tabular}


Table 6. Conodont samples from the Kogruk Formation-Continued.

[All faunas identified by A.G. Harris. Structural units determined by L.E. Young. EMA, Endicott Mountains allochthon; KRA, Kelly River allochthon. CAI, conodont color alteration index. Letters in field number refer to collector: AD, J.A. Dumoulin; AK, J.S. Kelley; AKD, K.D. Kelley; JS, J.M. Schmidt; KE, K. Evans; KS, K.W. Sherwood; MD, C.F. Mayfield; and TR, I.L. Tailleur. DDH, diamond drill hole. Interval thicknesses in drill holes are drilled thicknesses and have not been corrected for

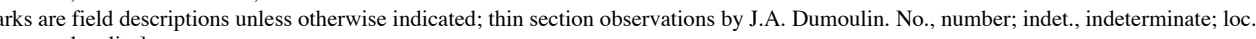
locality]

\begin{tabular}{|c|c|c|c|c|c|}
\hline $\begin{array}{l}\text { Locality No., } \\
\text { structural unit }\end{array}$ & $\begin{array}{l}\text { Quadrangle, } \\
\text { latitude n./ } \\
\text { longitude w. }\end{array}$ & $\begin{array}{c}\text { Conodont fauna and CAI } \\
\text { [field No.; USGS collection No.] }\end{array}$ & Age & $\begin{array}{c}\text { Conodont biofacies and } \\
\text { depositional } \\
\text { environment }\end{array}$ & Remarks \\
\hline $\begin{array}{l}6 \\
\text { KRA (Wulik } \\
\text { Peaks plate) }\end{array}$ & $\begin{array}{l}\text { De Long Mtns. } \\
\text { B-3 } \\
68^{\circ} 18^{\prime} 19.2^{\prime \prime} / \\
163^{\circ} 19^{\prime} 25^{\prime \prime}\end{array}$ & $\begin{array}{l}3 \text { well preserved Pa elements Bispathodus } \\
\text { utahensis Sandberg and Gutschick } \\
1 \text { large posterior Pa element fragment } \\
\text { Cavusgnathus sp. indet. } \\
\text { Kladognathus sp. } \\
2 \mathrm{M} \text { and } 2 \mathrm{Sb}-\mathrm{Sc} \text { elements } \\
\text { Synclydognathus geminus (Roundy) } \\
1 \mathrm{Sb} \text { and } 1 \mathrm{Sc} \text { elements } \\
11 \text { indet. bar, blade, and platform fragments } \\
\text { CAI=3 } \\
\text { [79MD146D; 27556-PC] }\end{array}$ & $\begin{array}{l}\text { middle Late Mississippian } \\
\text { (late Meramecian). }\end{array}$ & $\begin{array}{c}\text { Indeterminate (too few } \\
\text { conodonts); likely a } \\
\text { mix of shallow- and } \\
\text { deeper-water taxa. }\end{array}$ & Fossil loc. 29, Mayfield and others (1990). \\
\hline $\begin{array}{l}8 \\
\text { KRA (?Wulik } \\
\text { Peaks plate) }\end{array}$ & $\begin{array}{l}\text { De Long Mtns. } \\
\text { A-3 } \\
68^{\circ} 13^{\prime} 43.5^{\prime \prime} \\
163^{\circ} 13^{\prime} 01.2^{\prime \prime}\end{array}$ & $\begin{array}{l}1 \text { incomplete Pa element Bispathodus utahensis } \\
\text { Sandberg and Gutshick } \\
1 \text { anterior Pa element fragment Cavusgnathus sp. } \\
\text { indet. } \\
2 \text { incomplete Pa element fragments Hindeodus spp. } \\
27 \mathrm{~Pa} \text { elements Gnathodus homopunctatus Ziegler } \\
30 \mathrm{~Pa} \text { elements Gnathodus texanus Roundy } \\
\text { Synclydognathus geminus Hinde } \\
5 \mathrm{~Pa}, 2 \mathrm{~Pb} \text {, and } 16 \mathrm{~S} \text { elements } \\
\text { Unassigned elements: } \\
3 \mathrm{~Pb}(2 \text { morphotypes) and } 2 \mathrm{Sa} \text { ( } 2 \text { morphotypes) } \\
158 \text { indet. bar, blade, and platform fragments } \\
\mathrm{CAI}=2 \\
\text { [04AD39A; 33784-PC] }\end{array}$ & $\begin{array}{l}\text { middle Late Mississippian } \\
\text { (late Meramecian-very } \\
\text { early Chesterian; Lower } \\
\text { Cavusgnathus Zone into } \\
\text { succeeding Gn. bilineatus- } \\
\text { Upper Cavusgnathus Zone). }\end{array}$ & $\begin{array}{l}\text { Postmortem transport } \\
\text { from a gnathodid } \\
\text { biofacies. Outer shelf } \\
\text { or deeper water } \\
\text { depositional } \\
\text { environment }\end{array}$ & $\begin{array}{l}\text { Sample taken from uppermost well-exposed Lisburne } \\
\text { Group, underlying Etivluk Group and Okpikruak } \\
\text { Formation (Mayfield et al., 1990). } \\
\text { Sample from upper } 30 \mathrm{~cm} \text { of exposure, consists of } \\
\text { light-gray-weathering, medium-gray limestone with } \\
\text { sparse glauconite (skeletal grainstone with crinoids } \\
\text { and brachiopods) in 10- to 30-cm-thick beds. } \\
\text { Interval contains <10\% blackish brown, irregular } \\
\text { chert (replacing burrows?); upper part appears to be } \\
\text { bored. } \\
\text { Heavy-mineral concentrate is paleontologically diverse } \\
\text { and contains more than 95\% phosphatic and } \\
\text { phosphatized bioclasts (in order of decreasing } \\
\text { abundance: crinoid columnals and ossicles; } \\
\text { phosphatized ostracode, gastropod, and bivalve } \\
\text { steinkerns; phosphatic brachiopod fragments; } \\
\text { ichthyoliths; and phosphatized bryozoan fragments) } \\
\text { and rare glauconite. } \\
6.0 \text { kg of rock was processed }\end{array}$ \\
\hline $\begin{array}{l}21 \\
\text { KRA (Chimney } \\
\text { plate) }\end{array}$ & \begin{tabular}{|l} 
De Long Mtns. \\
A-3 \\
$68^{\circ} 08^{\prime} 39.2^{\prime \prime /}$ \\
$163^{\circ} 23^{\prime} 13^{\prime \prime}$
\end{tabular} & $\begin{array}{l}2 \text { Pa element fragments Polygnathus sp. indet. of } \\
\text { Middle Devonian-Early Mississippian } \\
\text { morphotype } \\
1 \mathrm{M} \text { (apatognathiform) element of Famennian-early } \\
\text { Chesterian morphotype } \\
8 \text { indet. bar, blade, and platform fragments } \\
\text { CAI=3.5 } \\
\text { [8-17-83C }]\end{array}$ & $\begin{array}{l}\text { late Late Devonian-Early } \\
\text { Mississippian (Famennian- } \\
\text { Osagean). }\end{array}$ & $\begin{array}{l}\text { Indeterminate (too few } \\
\text { conodonts). }\end{array}$ & $\begin{array}{l}\text { Kogruk Formation(?); collected near structural base of } \\
\text { thrust sheet. Dark-gray, thick-bedded micrite. Thin } \\
\text { section is fine-grained pelloidal-skeletal } \\
\text { grainstone/packstone; bioclasts include crinoids, } \\
\text { brachiopods, and ostracodes, as well as notable } \\
\text { calcispheres and girvanellid and other algae. } \\
5.34 \text { kg of rock was processed. } \\
\text { Sample collected by A.G. Harris. }\end{array}$ \\
\hline
\end{tabular}


Table 6. Conodont samples from the Kogruk Formation-Continued.

[All faunas identified by A.G. Harris. Structural units determined by L.E. Young. EMA, Endicott Mountains allochthon; KRA, Kelly River allochthon. CAI, conodont color alteration index. Letters in field number refer to collector: AD, J.A. Dumoulin; AK, J.S. Kelley; AKD, K.D. Kelley; JS, J.M. Schmidt; KE, K. Evans; KS, K.W. Sherwood; MD, C.F. Mayfield; and TR, I.L. Tailleur. DDH, diamond drill hole. Interval thicknesses in drill holes are drilled thicknesses and have not been corrected for e field desc locality]

\begin{tabular}{|c|c|c|c|c|c|}
\hline $\begin{array}{l}\text { Locality No., } \\
\text { structural unit }\end{array}$ & $\begin{array}{l}\text { Quadrangle, } \\
\text { latitude n./ } \\
\text { longitude w. }\end{array}$ & $\begin{array}{c}\text { Conodont fauna and CAI } \\
\text { [field No.; USGS collection No.] }\end{array}$ & Age & \begin{tabular}{|c|}
$\begin{array}{c}\text { Conodont biofacies and } \\
\text { depositional } \\
\text { environment }\end{array}$ \\
\end{tabular} & Remarks \\
\hline \multirow[t]{2}{*}{$\begin{array}{l}22 \\
\text { KRA (Chimney } \\
\text { plate) }\end{array}$} & \multirow[t]{2}{*}{$\begin{array}{l}\text { De Long Mtns. } \\
\text { A-3 } \\
68^{\circ} 08^{\prime} 30^{\prime \prime} \\
163^{\circ} 23^{\prime} 12^{\prime \prime}\end{array}$} & $\begin{array}{l}1 \text { Pa element fragment Bispathodus utahensis } \\
\text { Sandberg and Gutschick } \\
1 \text { Pa element Cavusgnathus sp. } \\
\text { Unassigned elements: } \\
1 \mathrm{~Pb}, 1 \mathrm{M} \text {, and } 1 \mathrm{Sb} \\
9 \text { indet. bar, blade, and platform fragments } \\
\text { CAI=3.5 } \\
\text { [8-17-83D; 29237-PC] }\end{array}$ & $\begin{array}{l}\text { probably middle Late } \\
\text { Mississippian (late } \\
\text { Meramecian-early } \\
\text { Chesterian(?), probably late } \\
\text { Meramecian). }\end{array}$ & $\begin{array}{l}\text { Indeterminate (too few } \\
\text { conodonts). }\end{array}$ & $\begin{array}{l}\text { Medium-dark-gray, light-gray-weathering, very fine } \\
\text { grained, slightly dolomitic limestone and chert } \\
\text { (limestone } 60 \% \text {, chert } 40 \% \text { ); } 50-100 \mathrm{ft} \text { above } 8-17- \\
83 \mathrm{C} \text { (loc. } 21 \text { ). Thin section is fine-grained pelloidal } \\
\text { calcisiltite; bioclasts include calcispheres, calcareous } \\
\text { spicules, rare crinoids, and ostracodes. Minor } \\
\text { scattered dolomite rhombs; trace quartz (and } \\
\text { feldspar?) silt along stylolites. } \\
\text { 5.04 kg of rock was processed. } \\
\text { Sample collected by A.G. Harris. }\end{array}$ \\
\hline & & $\begin{array}{l}2 \text { Pa elements Bispathodus utahensis Sandberg and } \\
\text { Gutschick } \\
\text { Cavusgnathus sp. indet. } \\
1 \mathrm{~Pa} \text { and } 1 \mathrm{M} \text { elements } \\
1 \mathrm{~Pb} \text { element Synclydognathus } \text { sp. indet. } \\
\text { Unassigned elements: } \\
1 \mathrm{Sa} \text { and } 1 \mathrm{Sc} \\
17 \text { indet. bar, blade, and platform fragments } \\
\text { CAI=3 } \\
\text { [8-17-83E; 29238-PC] }\end{array}$ & $\begin{array}{l}\text { probably middle Late } \\
\text { Mississippian (late } \\
\text { Meramecian-early } \\
\text { Chesterian(?), probably late } \\
\text { Meramecian). }\end{array}$ & $\begin{array}{l}\text { Indeterminate (too few } \\
\text { conodonts); probably } \\
\text { shallow to moderate } \\
\text { depth shelf or } \\
\text { platform. }\end{array}$ & $\begin{array}{l}\text { Sample taken a few hundred ft SE of 8-17-83D. } \\
\text { Massive, cliff-forming medium-gray, laminated, } \\
\text { siliceous limestone. Cliff is } 80 \% \text { blue-black chert } \\
\text { and } 20 \% \text { siliceous limestone. Thin section is } \\
\text { calcareous sponge spicule-pelloid packstone with } \\
\text { rare crinoid ossicles and radiolarians(?) and a trace } \\
\text { of quartz (and feldspar?) silt. } \\
6.64 \text { kg of rock was processed. } \\
\text { Sample collected by A.G. Harris. }\end{array}$ \\
\hline $\begin{array}{l}23 \\
\text { KRA (Chimney } \\
\text { plate) }\end{array}$ & $\begin{array}{l}\text { De Long Mtns. } \\
\text { A-3 } \\
68^{\circ} 07^{\prime} 44.6^{\prime \prime} / \\
163^{\circ} 23^{\prime} 25.2^{\prime \prime}\end{array}$ & $\begin{array}{l}6 \text { indet. bar, blade, and platform fragments } \\
\text { CAI }=4 \\
{[8-17-83 \mathrm{~F}]}\end{array}$ & Silurian-Permian & $\begin{array}{l}\text { Indeterminate (too few } \\
\text { conodonts). }\end{array}$ & $\begin{array}{l}\text { Dark-gray, fine-grained, crudely bedded limestone that } \\
\text { does not contain chert. Thin section is skeletal- } \\
\text { pelloidal packstone with diverse bioclasts, including } \\
\text { crinoid and brachiopod fragments, ostracodes, } \\
\text { foraminifers, and various algae; trace quartz and } \\
\text { feldspar silt. } \\
6.26 \text { kg of rock was processed. } \\
\text { Sample collected by A.G. Harris. }\end{array}$ \\
\hline $\begin{array}{l}32 \\
\text { KRA (Wulik } \\
\text { Peaks plate) }\end{array}$ & $\begin{array}{l}\text { De Long Mtns. } \\
\text { A-3 } \\
68^{\circ} 00^{\prime} 20.5^{\prime \prime} / \\
163^{\circ} 15^{\prime} 19.5^{\prime \prime}\end{array}$ & $\begin{array}{l}1 \text { Pa element Cavusgnathus unicornis Youngquist } \\
\text { and Miller } \\
1 \mathrm{~S} \text { element fragment Synclydognathus } \text { sp. indet. } \\
10 \text { indet. bar, blade, and platform fragments } \\
\text { CAI=2.5 } \\
\text { [79TR142; 27595-PC] }\end{array}$ & $\begin{array}{l}\text { middle Late Mississippian } \\
\text { (late Meramecian-early } \\
\text { Chesterian). }\end{array}$ & \begin{tabular}{|l|} 
Indeterminate (too few \\
conodonts). \\
Conodonts indicate \\
shallow-water, near a \\
restricted or \\
intermittently \\
restricted depositional \\
setting. \\
\end{tabular} & Fossil loc. 20, Mayfield and others (1990). \\
\hline
\end{tabular}


Table 6. Conodont samples from the Kogruk Formation-Continued.

[All faunas identified by A.G. Harris. Structural units determined by L.E. Young. EMA, Endicott Mountains allochthon; KRA, Kelly River allochthon. CAI, conodont color alteration index. Letters in field number refer to collector: AD, J.A. Dumoulin; AK, J.S. Kelley; AKD, K.D. Kelley; JS, J.M. Schmidt; KE, K. Evans; KS, K.W. Sherwood; MD, C.F. Mayfield; and TR, I.L. Tailleur. DDH, diamond drill hole. Interval thicknesses in drill holes are drilled thicknesses and have not been corrected for

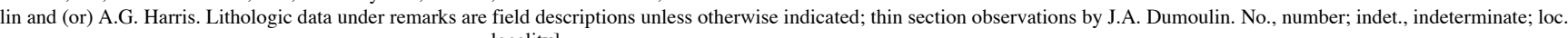
locality]

\begin{tabular}{|c|c|c|c|c|c|}
\hline $\begin{array}{l}\text { Locality No., } \\
\text { structural unit }\end{array}$ & $\begin{array}{l}\text { Quadrangle, } \\
\text { latitude n./ } \\
\text { longitude w. }\end{array}$ & $\begin{array}{c}\text { Conodont fauna and CAI } \\
\text { [field No.; USGS collection No.] }\end{array}$ & Age & $\begin{array}{c}\text { Conodont biofacies and } \\
\text { depositional } \\
\text { environment }\end{array}$ & Remarks \\
\hline \multirow[t]{2}{*}{$\begin{array}{l}40 \\
\text { KRA (Wulik } \\
\text { Peaks) }\end{array}$} & \multirow[t]{2}{*}{$\begin{array}{l}\text { De Long Mtns. } \\
\text { B-2 } \\
68^{\circ} 16^{\prime} 20.3^{\prime \prime} / \\
163^{\circ} 11^{\prime} 10.7^{\prime \prime}\end{array}$} & $\begin{array}{l}4 \text { Pa elements Bispathodus utahensis Sandberg and } \\
\text { Gutschick } \\
1 \text { Pa element Cavusgnathus unicornis Youngquist } \\
\text { and Miller } \\
2 \text { posterior Pa element fragments Cavusgnathus sp. } \\
\text { indet. } \\
1 \text { Pa element fragment Hindeodus sp. indet. or } \\
\text { Synclydognathus sp. indet. } \\
5 \text { Sb-Sc elements Kladognathus tenuis (Branson } \\
\text { and Mehl) } \\
29 \text { indet. bar, blade, and platform fragments } \\
\text { CAI=3 } \\
\text { [KE98-14D1; 33499-PC] }\end{array}$ & $\begin{array}{l}\text { middle Late Mississippian } \\
\text { (late Meramecian). }\end{array}$ & $\begin{array}{l}\text { Indeterminate (too few } \\
\text { generically } \\
\text { identifiable } \\
\text { conodonts). } \\
\text { Conodonts present } \\
\text { indicate postmortem } \\
\text { transport within or } \\
\text { from a shallow-water } \\
\text { depositional } \\
\text { environment. }\end{array}$ & $\begin{array}{l}\text { Float sample from lower part of Kogruk Formation(?); } \\
\text { see table } 7 \text { for Utukok Formation sample from this } \\
\text { locality. } \\
\text { Medium-gray-weathering, medium-dark-gray } \\
\text { limestone; crinoid-coral pack/wackestone. Thin } \\
\text { section is poorly sorted skeletal pack/wackestone } \\
\text { with trace quartz silt. Bioclasts consist of 30-50\% } \\
\text { crinoid ossicles, 5-15\% foraminifers, and lesser } \\
\text { algae, ostracodes, and bryozoan fragments. } \\
\text { Heavy-mineral concentrate includes phosphatized } \\
\text { bioclasts and rare ichthyoliths. } \\
3.9 \text { kg of rock was processed. }\end{array}$ \\
\hline & & $\begin{array}{l}2 \text { Pa element fragments Bispathodus utahensis } \\
\text { Sandberg and Gutschick } \\
1 \text { Pa fragment of a cavusgnathid } \\
\text { Kladognathus sp. indet. } \\
\text { 1 Sa and } 3 \text { Sc element fragments } \\
4 \text { indet. bar and blade fragments } \\
\text { CAI=3 } \\
\text { [KE98-14D2; 33500-PC] }\end{array}$ & $\begin{array}{l}\text { late Early-middle Late } \\
\text { Mississippian (late } \\
\text { Osagean-Meramecian). }\end{array}$ & $\begin{array}{l}\text { Indeterminate (too few } \\
\text { conodonts); } \\
\text { postmortem transport } \\
\text { within or from a } \\
\text { relatively shallow- } \\
\text { water depositional } \\
\text { setting. }\end{array}$ & $\begin{array}{l}\text { Outcrop sample, lower part of Kogruk Formation. } \\
\text { Limestone with irregular chert lenses and local } \\
\text { syringoporid corals. Thin section is crinoidal } \\
\text { packstone with notable foraminifers and lesser algae, } \\
\text { ostracodes, bryozoan fragments, and calcispheres. } \\
\text { Heavy-mineral concentrate consists chiefly of coarsely } \\
\text { phosphatized bioclasts (including pelmatozoans, } \\
\text { spine steinkerns, and bryozoans). } \\
4.32 \mathrm{~kg} \text { of rock was processed. }\end{array}$ \\
\hline $\begin{array}{l}2 \\
\text { EMA } \\
\text { (Wolverine } \\
\text { Creek plate; } \\
\text { Mt. Raven } \\
\text { window) }\end{array}$ & $\begin{array}{l}\text { De Long Mtns. } \\
\text { A-2 } \\
68^{\circ} 13^{\prime} 28.2^{\prime \prime} / \\
162^{\circ} 50^{\prime} 28.4^{\prime \prime}\end{array}$ & $\begin{array}{l}6 \text { Pa elements Gnathodus bilineatus (Roundy) } \\
\text { (early form) } \\
49 \mathrm{~Pa} \text { (juveniles to adults) elements Gnathodus } \\
\text { pseudosemiglaber Thompson and Fellows } \\
\text { Gnathodus sp. indet. } \\
4 \mathrm{~Pb}, 5 \mathrm{M}, 2 \mathrm{Sa} \text {, and } 3 \mathrm{Sc} \text { elements } \\
7 \text { Idioprioniodus sp. indet. element fragments } \\
\text { Lochriea commutata (Branson and Mehl) } \\
18 \mathrm{~Pa} \text { (all juveniles to subadults) and } 18 \mathrm{M} \\
\text { elements } \\
1 \mathrm{M} \text { element Kladognathus sp. indet. } \\
311 \text { indet. bar, blade, and platform fragments } \\
\text { CAI=4 } \\
\text { [99AD13I; 33480-PC] }\end{array}$ & $\begin{array}{l}\text { middle Late Mississippian } \\
\text { (late Meramecian-early } \\
\text { Chesterian). }\end{array}$ & $\begin{array}{l}\text { Postmortem transport } \\
\text { within the gnathodid- } \\
\text { lochrieid biofacies; } \\
\text { conodonts indicate a } \\
\text { slope depositional } \\
\text { setting. }\end{array}$ & $\begin{array}{l}\text { Sample from stratigraphically highest carbonate bed in } \\
\text { Lisburne Group here, which underlies several meters } \\
\text { of black chert (uppermost Lisburne), which underlies } \\
\text { white-weathering radiolarian chert (basal Siksikpuk } \\
\text { Formation). } \\
\text { Carbonate layer is } 1 \text { m thick, medium dark gray, very } \\
\text { light gray to pinkish gray weathering, fine grained, } \\
\text { very fetid, and contains possible peloids. It forms } \\
\text { uneven } 15 \text { to } 20 \mathrm{~cm} \text {-thick beds with nodules of black } \\
\text { vitreous chert to } 60 \mathrm{~cm} \text { long. Thin section is organic- } \\
\text { rich, fine-grained, euhedral dolomite(?) mosaic, with } \\
\text { rare bioclasts. } \\
\text { Heavy-mineral concentrate includes phosphatized } \\
\text { peloids(?) and composite phosphatized grains, as } \\
\text { well as lesser phosphatized steinkerns of gastropods, } \\
\text { ostracodes, pelecypods, and spines. } \\
11.4 \text { kg of rock was processed. } \\
\text { Loc. } 4 \text { (fig. 2) of Dumoulin and others (2004). }\end{array}$ \\
\hline
\end{tabular}


Table 6. Conodont samples from the Kogruk Formation-Continued.

[All faunas identified by A.G. Harris. Structural units determined by L.E. Young. EMA, Endicott Mountains allochthon; KRA, Kelly River allochthon. CAI, conodont color alteration index. Letters in field number refer to collector: AD, J.A. Dumoulin; AK, J.S. Kelley; AKD, K.D. Kelley; JS, J.M. Schmidt; KE, K. Evans; KS, K.W. Sherwood; MD, C.F. Mayfield; and TR, I.L. Tailleur. DDH, diamond drill hole. Interval thicknesses in drill holes are drilled thicknesses and have not been corrected for locality]

\begin{tabular}{|c|c|c|c|c|c|}
\hline $\begin{array}{l}\text { Locality No., } \\
\text { structural unit }\end{array}$ & $\begin{array}{l}\text { Quadrangle, } \\
\text { latitude n./ } \\
\text { longitude w. }\end{array}$ & $\begin{array}{c}\text { Conodont fauna and CAI } \\
\text { [field No.; USGS collection No.] }\end{array}$ & Age & $\begin{array}{c}\text { Conodont biofacies and } \\
\text { depositional } \\
\text { environment }\end{array}$ & Remarks \\
\hline $\begin{array}{l}43 \\
\text { EMA } \\
\text { (Wolverine } \\
\text { Creek plate; } \\
\text { Mt. Raven } \\
\text { window) }\end{array}$ & $\begin{array}{l}\text { De Long Mtns } \\
\text { A-2 } \\
68^{\circ} 13^{\prime} 18^{\prime \prime} / \\
162^{\circ} 44^{\prime} 18^{\prime \prime}\end{array}$ & $\begin{array}{l}\text { Barren } \\
{[\text { KS98-105] }}\end{array}$ & & & $\begin{array}{l}\text { Sample submitted as Utukok Formation(?) but facies } \\
\text { and position suggest upper part of Kogruk } \\
\text { Formation. Strata are } \sim 10 \mathrm{~m} \text { south of Etivluk Group; } \\
\text { contact between the two units may be a fault. } \\
\text { Rusty-brown dolomite or impure limestone } \\
\text { interbedded with dark-gray shale. Thin section is } \\
\text { cherty fine-grained dolomitic limestone with rare } \\
\text { radiolarians (some filled with chalcedony, others } \\
\text { with carbonate) and calcareous and siliceous } \\
\text { spicules. } \\
12.2 \mathrm{~kg} \text { of rock was processed. } \\
\end{array}$ \\
\hline $\begin{array}{l}44 \\
\text { EMA } \\
\text { (Wolverine } \\
\text { Creek plate; } \\
\text { Mt. Raven } \\
\text { window) }\end{array}$ & $\begin{array}{l}\text { De Long Mtns. } \\
\text { A-2 } \\
68^{\circ} 12^{\prime} 35.9^{\prime \prime /} \\
162^{\circ} 52^{\prime} 48^{\prime \prime}\end{array}$ & $\begin{array}{l}\text { Barren } \\
\text { [KE98-27] }\end{array}$ & & & $\begin{array}{l}\text { Light-gray dolostone. Thin section is finely crystalline } \\
\text { euhedral/subhedral dolomite mosaic with } \sim 10 \% \\
\text { quartz that occurs between crystals and fills vugs and } \\
\text { rare molds of crinoid ossicles. Some vugs contain } \\
\text { dead oil. } \\
\text { Heavy-mineral concentrate includes indeterminate } \\
\text { phosphatized bioclasts. } \\
2.94 \mathrm{~kg} \text { of rock was processed. }\end{array}$ \\
\hline $\begin{array}{l}45 \\
\text { EMA } \\
\text { (Wolverine } \\
\text { Creek plate; } \\
\text { Mt. Raven } \\
\text { window) }\end{array}$ & $\begin{array}{l}\text { De Long Mtns. } \\
\text { A-2 } \\
68^{\circ} 12^{\prime} 30^{\prime \prime} \\
162^{\circ} 53^{\prime} 32^{\prime \prime}\end{array}$ & $\begin{array}{l}\text { Cavusgnathus sp. indet. } \\
1 \mathrm{M} \text { and } 3 \mathrm{~Pa} \text { element fragments } \\
1 \mathrm{Sc} \text { element Hindeodus sp. indet. } \\
\text { Kladognathus sp. } \\
3 \mathrm{M} \text { and } 3 \mathrm{Sb}-\mathrm{Sc} \text { elements } \\
\text { Synclydognathus geminus (Hinde) } \\
3 \mathrm{~Pa}, 1 \mathrm{M}, 1 \mathrm{Sa} \text {, and } 1 \mathrm{Sb} \text { elements } \\
74 \text { chiefly small indet. bar, blade, and platform } \\
\text { fragments } \\
\text { CAI=3 } \\
\text { [98AD9A; 33415-PC] }\end{array}$ & $\begin{array}{l}\text { middle Late Mississippian } \\
\text { (late Meramecian-early } \\
\text { Chesterian). }\end{array}$ & $\begin{array}{l}\text { Indeterminate (too few } \\
\text { conodonts). } \\
\text { Postmortem mixture } \\
\text { of species } \\
\text { representing shallow- } \\
\text { water, near restricted } \\
\text { depositional setting. }\end{array}$ & $\begin{array}{l}\text { Sample taken several hundred feet stratigraphically } \\
\text { below top of Kogruk Formation. } \\
\text { Subcrop of massive, light-gray, crinoidal dolostone } \\
\text { (packstone/grainstone) with irregular stringers of } \\
\text { black chert. Thin section is dolostone (crystals } 20 \\
\mu \mathrm{m} \text { to }>1 \mathrm{~mm} \text {, most } 100-200 \mu \mathrm{m} \text { ) with relict } \\
\text { pelmatozoan fragments } 1 \text { to } 7 \mathrm{~mm} \text { in diameter; } \\
\text { abundant vugs (1 to } 4 \mathrm{~mm} \text { ), some of which look like } \\
\text { molds of crinoid ossicles. } \\
10.3 \mathrm{~kg} \text { of rock was processed. } \\
\text { Loc. } 5 \text { (fig. } 2 \text { ) of Dumoulin and others (2004). }\end{array}$ \\
\hline
\end{tabular}


Table 6. Conodont samples from the Kogruk Formation-Continued.

[All faunas identified by A.G. Harris. Structural units determined by L.E. Young. EMA, Endicott Mountains allochthon; KRA, Kelly River allochthon. CAI, conodont color alteration index. Letters in field number refer to collector: AD, J.A. Dumoulin; AK, J.S. Kelley; AKD, K.D. Kelley; JS, J.M. Schmidt; KE, K. Evans; KS, K.W. Sherwood; MD, C.F. Mayfield; and TR, I.L. Tailleur. DDH, diamond drill hole. Interval thicknesses in drill holes are drilled thicknesses and have not been corrected for 作 field descriptions unless otherwise indicated; thin section observations by J.A. Dumoulin. No., number; indet, indeterminate; loc., locality]

\begin{tabular}{|c|c|c|c|c|c|}
\hline $\begin{array}{l}\text { Locality No., } \\
\text { structural unit }\end{array}$ & $\begin{array}{l}\text { Quadrangle, } \\
\text { latitude } \mathrm{n} . / \\
\text { longitude w. }\end{array}$ & $\begin{array}{c}\text { Conodont fauna and CAI } \\
\text { [field No.; USGS collection No.] }\end{array}$ & Age & \begin{tabular}{|c|}
$\begin{array}{c}\text { Conodont biofacies and } \\
\text { depositional } \\
\text { environment }\end{array}$ \\
\end{tabular} & Remarks \\
\hline $\begin{array}{l}46 \\
\text { KRA (Wulik } \\
\text { Peaks plate) }\end{array}$ & $\begin{array}{l}\text { De Long Mtns. } \\
\text { A-2 } \\
68^{\circ} 13^{\prime} 38^{\prime \prime} / \\
163^{\circ} 04^{\prime} 14.2^{\prime \prime}\end{array}$ & $\begin{array}{l}3 \text { juvenile Pa elements Cavusgnathus unicornis } \\
\text { Youngquist and Miller } \\
1 \text { juvenile Pa element Gnathodus texanus Roundy } \\
2 \text { Sb elements Synclydognathus sp. indet. } \\
\text { Unassigned elements: } \\
2 \mathrm{~Pb}, 2 \mathrm{M} \text { ( } 2 \text { morphotypes), } 1 \mathrm{Sa} \text {, and } 2 \mathrm{Sc} \text { (two } \\
\text { morphotypes) } \\
\text { CAI=3 } \\
\text { [8-16-83D; 29230-PC] }\end{array}$ & $\begin{array}{l}\text { middle Late Mississippian } \\
\text { (late Meramecian-early } \\
\text { Chesterian). }\end{array}$ & $\begin{array}{l}\text { Indeterminate (too few } \\
\text { conodonts and mixed } \\
\text { biofacies). }\end{array}$ & $\begin{array}{l}\text { Medium-gray, fine- to medium-grained, medium- to } \\
\text { thick-bedded, bioclastic, bioturbated wackestone } \\
\text { containing } ~ 20-30 \% \text { chert. Thin section is } \\
\text { overpacked, skeletal-pelloidal packstone; bioclasts } \\
\text { (80\% of grains) include pelmatozoan, bryozoan, and } \\
\text { brachiopod fragments and articulated ostracodes. } \\
\text { Minor nonferroan dolomite and detrital quartz silt. } \\
6.4 \text { kg of rock was processed. } \\
\text { Sample collected by A.G. Harris. }\end{array}$ \\
\hline $\begin{array}{l}56 \\
\text { KRA (Wulik } \\
\text { Peaks) }\end{array}$ & $\begin{array}{l}\text { De Long Mtns. } \\
\text { A-2 } \\
68^{\circ} 11^{\prime} 34.5^{\prime \prime /} \\
163^{\circ} 10^{\prime} 0.8^{\prime \prime}\end{array}$ & $\begin{array}{l}\text { 1 Sa element Kladognathus sp. indet. } \\
1 \text { unassigned robust digyrate Sc element } \\
1 \text { indet. bar fragment } \\
\text { CAI }=2.5 \\
\text { [KE98-13; 33498-PC] }\end{array}$ & Mississippian & $\begin{array}{l}\text { Indeterminate; too few } \\
\text { conodonts. }\end{array}$ & $\begin{array}{l}\text { Near top of Kogruk Formation here. See table } 7 \text { for } \\
\text { Utukok Formation sample from this locality. } \\
\text { Medium-gray limestone with crinoid ossicles. Thin } \\
\text { section is skeletal supportstone with locally } \\
\text { dolomitized matrix; bioclasts include pelmatozoan, } \\
\text { bryozoan, ostracode, and brachiopod fragments, } \\
\text { foraminifers, and diverse algae. } \\
\text { Heavy-mineral concentrate is chiefly phosphatized } \\
\text { rock fragments and minor bioclasts, ichthyoliths, and } \\
\text { anhedral pyrite and chalcopyrite. } \\
6.1 \text { kg of rock was processed. } \\
\end{array}$ \\
\hline $\begin{array}{l}58 \\
\text { KRA? }\end{array}$ & $\begin{array}{l}\text { De Long Mtns. } \\
\text { A-2 } \\
68^{\circ} 11^{\prime} 27.6^{\prime \prime /} \\
163^{\circ} 08^{\prime} 24^{\prime \prime}\end{array}$ & $\begin{array}{l}1 \text { indet. bar fragment } \\
\text { CAI=3 } \\
\text { [98JS8E; 33611-PC] }\end{array}$ & Mississippian & $\begin{array}{l}\text { Indeterminate (too few } \\
\text { conodonts). }\end{array}$ & $\begin{array}{l}\text { Undolomitized Kogruk Formation. Thin section is } \\
\text { fine-grained skeletal grainstone with foraminifers, } \\
\text { algae, crinoid ossicles and articulated ostracode } \\
\text { valves. } \\
5.8 \mathrm{~kg} \text { of rock was processed. } \\
\end{array}$ \\
\hline $\begin{array}{l}60 \\
\text { KRA (Wulik } \\
\text { Peaks) }\end{array}$ & $\begin{array}{l}\text { De Long Mtns. } \\
\text { A-2 } \\
68^{\circ} 11^{\prime} 09^{\prime \prime} / \\
163^{\circ} 11^{\prime} 19.5^{\prime \prime}\end{array}$ & $\begin{array}{l}6 \text { juvenile Pa elements Gnathodus } \\
\text { pseudosemiglaber Bischoff } \\
8 \text { juvenile or fragmentary Pa elements Gnathodus } \\
\text { sp. indet. } \\
\text { Unassigned elements: } \\
1 \mathrm{Sa} \text { and } 2 \mathrm{Sc} \\
92 \text { small indet. bar, blade, and platform fragments } \\
\text { CAI=3 } \\
\text { [KE98-12; 33497-PC] }\end{array}$ & $\begin{array}{l}\text { late Early-middle Late } \\
\text { Mississippian (middle } \\
\text { Osagean-Meramecian; } \\
\text { middle } S c \text {. anchoralis-Do. } \\
\text { latus Zone through } \\
\text { Meramecian). }\end{array}$ & $\begin{array}{l}\text { Indeterminate (too few } \\
\text { generically } \\
\text { identifiable } \\
\text { conodonts). } \\
\text { Conodonts present } \\
\text { suggest postmortem } \\
\text { winnow in an open- } \\
\text { marine setting. }\end{array}$ & $\begin{array}{l}\text { Top of Kogruk Formation here. } \\
\text { Light-gray-weathering, medium-light-gray, fine- } \\
\text { grained limy dolostone. Thin section is dolomitic } \\
\text { crinoid wacke/packstone; some crinoids are still } \\
\text { calcite, but others are replaced by silica. } \\
\text { Heavy-mineral concentrate includes minor phosphatic } \\
\text { brachiopod fragments and rare ichthyoliths. } \\
5.8 \text { kg of rock was processed. }\end{array}$ \\
\hline
\end{tabular}


Table 6. Conodont samples from the Kogruk Formation-Continued.

[All faunas identified by A.G. Harris. Structural units determined by L.E. Young. EMA, Endicott Mountains allochthon; KRA, Kelly River allochthon. CAI, conodont color alteration index. Letters in field number refer to collector: AD, J.A. Dumoulin; AK, J.S. Kelley; AKD, K.D. Kelley; JS, J.M. Schmidt; KE, K. Evans; KS, K.W. Sherwood; MD, C.F. Mayfield; and TR, I.L. Tailleur. DDH, diamond drill hole. Interval thicknesses in drill holes are drilled thicknesses and have not been corrected for

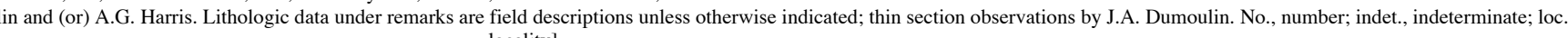
locality]

\begin{tabular}{|c|c|c|c|c|c|}
\hline $\begin{array}{l}\text { Locality No., } \\
\text { structural unit }\end{array}$ & $\begin{array}{l}\text { Quadrangle, } \\
\text { latitude n./ } \\
\text { longitude w. }\end{array}$ & $\begin{array}{c}\text { Conodont fauna and CAI } \\
\text { [field No.; USGS collection No.] }\end{array}$ & Age & \begin{tabular}{|c|}
$\begin{array}{c}\text { Conodont biofacies and } \\
\text { depositional } \\
\text { environment }\end{array}$ \\
\end{tabular} & Remarks \\
\hline $\begin{array}{l}62 \\
\text { KRA (Wulik } \\
\text { Peaks plate) }\end{array}$ & $\begin{array}{l}\text { De Long Mtns. } \\
\text { A-2 } \\
68^{\circ} 10^{\prime} 51^{\prime \prime} / \\
163^{\circ} 10^{\prime} 25.6^{\prime \prime}\end{array}$ & $\begin{array}{l}1 \text { juvenile Pa element Cavusgnathus unicornis } \\
\text { Youngquist and Miller } \\
2 \text { indet. bar and blade fragments } \\
\text { CAI }=4 \text { ? } \\
\text { [98JS7K; 33609-PC] }\end{array}$ & $\begin{array}{l}\text { Late, but not earliest Late } \\
\text { Mississippian (late } \\
\text { Meramecian-Chesterian). }\end{array}$ & $\begin{array}{l}\text { Indeterminate (too few } \\
\text { conodonts). }\end{array}$ & $\begin{array}{l}\text { Top of Kogruk Formation here. See tables } 7 \text { and } 9 \text { for } \\
\text { additional samples from this locality. } \\
\text { Light-gray limestone. Thin section is coarse-grained, } \\
\text { packed, skeletal grainstone; main bioclasts are } \\
\text { crinoid ossicles, bryozoans, and brachiopod } \\
\text { fragments with lesser articulated, thick-shelled } \\
\text { ostracodes and trace quartz and plagioclase feldspar } \\
\text { silt. } \\
\text { Heavy-mineral concentrate includes phosphatized } \\
\text { composite grains, peloids, and bioclasts. } \\
5.7 \text { kg of rock was processed. }\end{array}$ \\
\hline $\begin{array}{l}68 \\
\text { EMA } \\
\text { (Wolverine } \\
\text { Creek plate; } \\
\text { Mt. Raven } \\
\text { window) }\end{array}$ & $\begin{array}{l}\text { De Long Mtns. } \\
\text { A-2 } \\
68^{\circ} 10^{\prime} 42^{\prime \prime} / \\
162^{\circ} 41^{\prime} 12^{\prime \prime}\end{array}$ & $\begin{array}{l}2 \text { unassigned Sc element fragments ( } 2 \\
\text { morphotypes) } \\
11 \text { indet. bar, blade, and platform fragments } \\
\text { CAI }=3 \\
{[\mathrm{KS} 98-90 ; 33607-\mathrm{PC}]}\end{array}$ & Post-Ordovician Paleozoic & $\begin{array}{l}\text { Indeterminate (too few } \\
\text { conodonts). }\end{array}$ & $\begin{array}{l}\text { Sample near base of Kogruk Formation, which is here } \\
\text { thrust above Etivluk Group. } \\
\text { Medium- to medium-light-gray, finely laminated } \\
\text { dolostone }(40 \%) \text { and black chert }(60 \%) \text { in even beds } \\
2 \text { to } 3 \mathrm{~cm} \text { thick. Thin section is finely crystalline } \\
\text { euhedral to subhedral dolomite mosaic with rare } \\
\text { relict crinoid ossicles(?). } \\
6.82 \mathrm{~kg} \text { of rock was processed. }\end{array}$ \\
\hline $\begin{array}{l}69 \\
\text { KRA (Kelly } \\
\text { plate) }\end{array}$ & $\begin{array}{l}\text { De Long Mtns. } \\
\text { A-2 } \\
68^{\circ} 09^{\prime} 41^{\prime \prime} \\
162^{\circ} 45^{\prime} 10^{\prime \prime}\end{array}$ & $\begin{array}{l}5 \text { Pa element fragments Cavusgnathus unicornis } \\
\text { Youngquist and Miller } \\
\text { Hindeodus sp. indet. } \\
3 \mathrm{~Pa} \text { and } 1 \text { Sa elements (all fragments) } \\
1 \mathrm{Sb}-\mathrm{Sc} \text { element Kladognathus sp. indet. } \\
48 \text { indet. bar, blade, and platform fragments } \\
\text { CAI=2.5-3 } \\
\text { [98AD6E; 33414-PC] }\end{array}$ & \begin{tabular}{|l|} 
middle-late Late \\
Mississippian (late \\
Meramecian-Chesterian).
\end{tabular} & $\begin{array}{l}\text { Indeterminate (too few } \\
\text { conodonts). } \\
\text { Conodont fragments } \\
\text { present indicate } \\
\text { derivation from a } \\
\text { shallow-water } \\
\text { depositional } \\
\text { environment. }\end{array}$ & $\begin{array}{l}\text { Near contact with Utukok Formation as mapped by } \\
\text { Ellersieck and others (1990); probably in lower third } \\
\text { of Kogruk Formation. } \\
\text { Massive, light-gray-weathering, medium-dark-gray } \\
\text { limestone with scattered fossils (mostly crinoids) and } \\
20-30 \% \text { chert. Thin section is peloidal-skeletal } \\
\text { packstone to grainstone with minor detrital quartz } \\
\text { sand and abundant stylolites and fractures; many } \\
\text { bioclasts are micritized. } \\
8.9 \text { kg of rock was processed. }\end{array}$ \\
\hline $\begin{array}{l}73 \\
\text { EMA } \\
\text { (Wolverine } \\
\text { Creek plate; } \\
\text { Rok window) }\end{array}$ & $\begin{array}{l}\text { De Long Mtns. } \\
\text { A-2 } \\
68^{\circ} 07^{\prime} 00^{\prime \prime} / \\
163^{\circ} 08^{\prime} 36^{\prime \prime}\end{array}$ & $\begin{array}{l}5 \text { Pa elements Gnathodus pseudosemiglaber } \\
\text { Thompson and Fellows } \\
21 \text { Pa elements Gnathodus texanus Roundy } \\
13 \text { Pa element fragments Gnathodus spp.? indet. } \\
1 \text { Pa element fragment Rhachistognathus prolixus } \\
\text { Baesemann and Lane } \\
202 \text { indet. bar, blade, and platform fragments } \\
\text { CAI=2.5 } \\
\text { [KE98-25A; 33603-PC] }\end{array}$ & $\begin{array}{l}\text { Likely middle Late } \\
\text { Mississippian (very early } \\
\text { Chesterian). }\end{array}$ & $\begin{array}{l}\text { Postmortem transport } \\
\text { from or within the } \\
\text { gnathodid biofacies. } \\
\text { Outer shelf or deeper } \\
\text { water depositional } \\
\text { environment. }\end{array}$ & $\begin{array}{l}\text { Uppermost Kogruk Formation; see table } 7 \text { for Utukok } \\
\text { Formation sample from this locality. } \\
\text { Sample from top of cliff. Partly dolomitized skeletal } \\
\text { grainstone interbedded with black chert. Thin section } \\
\text { is euhedral/subhedral dolomite mosaic with as much } \\
\text { as } 30 \% \text { quartz that occurs between crystals and fills } \\
\text { vugs and rare molds of crinoid ossicles. Some vugs } \\
\text { contain dead oil. } \\
\text { Heavy-mineral concentrate includes dolomitized and } \\
\text { phosphatized composite grains and bioclasts } \\
\text { (bryozoans and pelmatozoans). } \\
5.1 \text { kg of rock was processed. } \\
\text { Loc. } 14 \text { (fig. 2) of Dumoulin and others (2004). } \\
\end{array}$ \\
\hline
\end{tabular}


Table 6. Conodont samples from the Kogruk Formation-Continued.

[All faunas identified by A.G. Harris. Structural units determined by L.E. Young. EMA, Endicott Mountains allochthon; KRA, Kelly River allochthon. CAI, conodont color alteration index. Letters in field number refer to collector: AD, J.A. Dumoulin; AK, J.S. Kelley; AKD, K.D. Kelley; JS, J.M. Schmidt; KE, K. Evans; KS, K.W. Sherwood; MD, C.F. Mayfield; and TR, I.L. Tailleur. DDH, diamond drill hole. Interval thicknesses in drill holes are drilled thicknesses and have not been corrected for re field descriptions unless otherwise indicated; thin section observations by J.A. Dumoulin. No., number; indet., indeterminate; loc. locality]

\begin{tabular}{|c|c|c|c|c|c|}
\hline $\begin{array}{l}\text { Locality No., } \\
\text { structural unit }\end{array}$ & $\begin{array}{l}\text { Quadrangle, } \\
\text { latitude } \mathrm{n} . / \\
\text { longitude w. }\end{array}$ & $\begin{array}{c}\text { Conodont fauna and CAI } \\
\text { [field No.; USGS collection No.] }\end{array}$ & Age & \begin{tabular}{|c|} 
Conodont biofacies and \\
depositional \\
environment \\
\end{tabular} & Remarks \\
\hline $\begin{array}{l}73 \\
\text { EMA } \\
\text { (Wolverine } \\
\text { Creek plate; } \\
\text { Rok window) } \\
\text { [cont.] }\end{array}$ & $\begin{array}{l}\text { De Long Mtns. } \\
\text { A-2 } \\
68^{\circ} 07^{\prime} 00^{\prime \prime} / \\
163^{\circ} 08^{\prime} 36^{\prime \prime}\end{array}$ & $\begin{array}{l}\text { Kladognathus sp. } \\
6 \mathrm{M}, 1 \mathrm{Sa} \text {, and } 16 \mathrm{Sb}-\mathrm{Sc} \text { elements } \\
7 \mathrm{~Pa} \text { elements Polygnathus mehli Thompson [pl. 2, } \\
\text { figs. } 14,15] \\
\text { Synclydognathus geminus (Hinde) } \\
2 \mathrm{~Pa} \text { and } 5 \mathrm{M} \text { and } \mathrm{S} \text { elements (chiefly fragments) } \\
\text { Unassigned elements: } \\
2 \mathrm{~Pb}(2 \text { morphotypes) and } 2 \mathrm{Sc} \text { ( } 2 \text { morphotypes) } \\
26 \text { indet. bar, blade, and platform fragments } \\
\text { CAI=3 } \\
{[\text { KE98-25B; 33604-PC] }}\end{array}$ & $\begin{array}{l}\text { late Early Mississippian (late } \\
\text { middle-earliest late } \\
\text { Osagean; uppermost } S c \text {. } \\
\text { anchoralis-Do. latus Zone } \\
\text { into succeeding earliest Po. } \\
\text { mehli-Lower Gn. texanus } \\
\text { Zone). }\end{array}$ & $\begin{array}{l}\text { Postmortem transport } \\
\text { from or within the } \\
\text { kladognathid- } \\
\text { polygnathid biofacies. } \\
\text { Shelfal depositional } \\
\text { setting. }\end{array}$ & $\begin{array}{l}\text { Sample taken at base of cliff, } 220 \mathrm{ft} \text { below KE98- } \\
25 \mathrm{~A} \text {. } \\
\text { Light-gray-weathering, medium-gray limestone with } \\
\text { crinoid ossicles and local corals. Thin section is } \\
\text { skeletal packstone with dolomitized matrix; bioclasts } \\
\text { include pelmatozoans, bryozoans, and lesser } \\
\text { brachiopods, ostracodes, algae, foraminifers, and } \\
\text { calcareous spicules. } \\
\text { Heavy-mineral concentrate includes dolomitized } \\
\text { indeterminate bioclasts, rare phosphatic brachiopod } \\
\text { fragments, and ichthyoliths. } \\
3.7 \mathrm{~kg} \text { of rock was processed. }\end{array}$ \\
\hline $\begin{array}{l}75 \\
\text { EMA } \\
\text { (Wolverine } \\
\text { Creek plate; } \\
\text { Rok window) }\end{array}$ & $\begin{array}{l}\text { De Long Mtns. } \\
\text { A-2 } \\
68^{\circ} 05^{\prime} 54^{\prime \prime} / \\
163^{\circ} 0657^{\prime \prime}\end{array}$ & $\begin{array}{l}\text { Conodonts are mostly fragments-breakage is } \\
\text { mainly due to brittle deformation and is not } \\
\text { depositional. } \\
11 \text { Pa elements Cavusgnathus unicornis } \\
\text { Youngquist and Miller } \\
6 \text { largely incomplete Pa elements Hindeodus } \\
\text { minutus (Ellison) } \\
\text { Kladognathus tenuis } \\
2 \text { Pa-Pb (fragments), } 7 \mathrm{M}, 14 \text { Sb-Sc elements } \\
20 \text { Pa elements Rhachistognathus prolixus } \\
\text { Baesemann and Lane } \\
\text { Synclydognathus geminus (Hinde) } \\
11 \text { Pa and } 12 \mathrm{~S} \text { and M elements } \\
\text { Unassigned elements: } \\
4 \mathrm{M}(3 \text { morphotypes) and } 1 \mathrm{Sa} \\
236 \text { indet. bar, blade, and platform fragments } \\
\text { CAI=3.5-4 } \\
\text { [00AD21A; 33632-PC] }\end{array}$ & $\begin{array}{l}\text { middle Late Mississippian, } \\
\text { (very early Chesterian). }\end{array}$ & $\begin{array}{l}\text { Synclydognathid- } \\
\text { cavusgnathid } \\
\text { biofacies; shallow- } \\
\text { water, near high- } \\
\text { energy depositional } \\
\text { setting. }\end{array}$ & $\begin{array}{l}\text { About } 10 \mathrm{~m} \text { below top of Kogruk Formation at } \\
\text { stratigraphic contact with Siksikpuk Formation. } \\
\text { Light-gray- to very light gray-weathering, medium- } \\
\text { gray and pinkish-gray, massively bedded, finely to } \\
\text { moderately sucrosic cherty dolostone in beds } 0.7 \text { to } 1 \\
\text { m thick; chert randomly distributed in irregular } \\
\text { masses } 10 \text { to } 30 \mathrm{~cm} \text { in size. Some areas } \\
\text { autobrecciated. Part of large continuous section } \\
\text { along cutbank of Wulik River. Thin section is } \\
\text { dolostone with relict crinoid ossicles. } \\
\text { Heavy-mineral concentrate contains phosphatized } \\
\text { bioclasts (including phosphatized pelmatozoan } \\
\text { ossicles, gastropod steinkerns, bryozoan zooecial } \\
\text { fillings, and spines) and phosphatic bioclasts } \\
\text { (ichthyoliths). } \\
11.5 \mathrm{~kg} \text { of rock was processed. } \\
\text { Loc. } 15 \text { (fig. 2) of Dumoulin and others (2004). }\end{array}$ \\
\hline
\end{tabular}


Table 6. Conodont samples from the Kogruk Formation-Continued.

[All faunas identified by A.G. Harris. Structural units determined by L.E. Young. EMA, Endicott Mountains allochthon; KRA, Kelly River allochthon. CAI, conodont color alteration index. Letters in field number refer to collector: AD, J.A. Dumoulin; AK, J.S. Kelley; AKD, K.D. Kelley; JS, J.M. Schmidt; KE, K. Evans; KS, K.W. Sherwood; MD, C.F. Mayfield; and TR, I.L. Tailleur. DDH, diamond drill hole. Interval thicknesses in drill holes are drilled thicknesses and have not been corrected for

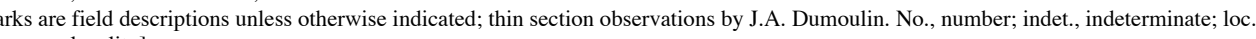
locality]

\begin{tabular}{|c|c|c|c|c|c|}
\hline $\begin{array}{l}\text { Locality No., } \\
\text { structural unit }\end{array}$ & $\begin{array}{l}\text { Quadrangle, } \\
\text { latitude n./ } \\
\text { longitude w. }\end{array}$ & $\begin{array}{c}\text { Conodont fauna and CAI } \\
\text { [field No.; USGS collection No.] }\end{array}$ & Age & $\begin{array}{c}\text { Conodont biofacies and } \\
\text { depositional } \\
\text { environment }\end{array}$ & Remarks \\
\hline $\begin{array}{l}82 \\
\text { EMA } \\
\text { (Wolverine } \\
\text { Creek plate; } \\
\text { Rok window) }\end{array}$ & $\begin{array}{l}\text { De Long Mtns. } \\
\text { A-2 } \\
68^{\circ} 01^{\prime} 30^{\prime \prime} / \\
163^{\circ} 01^{\prime} 48^{\prime \prime}\end{array}$ & $\begin{array}{l}2 \text { Pa element fragments Bispathodus stabilis } \\
\text { (Branson and Mehl) or Bi. utahensis Sandberg } \\
\text { and Gutschick } \\
4 \text { Pa element fragments Bispathodus sp. indet. } \\
\text { (double-row) } \\
1 \text { unassigned M element } \\
19 \text { indet. bar, blade, and platform fragments } \\
\text { CAI=3.5-4 } \\
\text { [8-15-83B; 29227-PC] }\end{array}$ & $\begin{array}{l}\text { late Late Devonian- early } \\
\text { Early Mississippian (late } \\
\text { Famennian-Kinderhookian) }\end{array}$ & $\begin{array}{l}\text { Indeterminate (too few } \\
\text { conodonts); conodonts } \\
\text { derived from a } \\
\text { normal-marine, } \\
\text { probably shallow- } \\
\text { water depositional } \\
\text { setting. }\end{array}$ & $\begin{array}{l}\text { Upper part of Kogruk Formation. Age atypically old } \\
\text { for Kogruk; conodonts may have been reworked } \\
\text { from underlying older rocks. } \\
\text { Medium-gray to light-brownish-gray, light-gray- } \\
\text { weathering, siliceous, very fine grained, laminated } \\
\text { dolostone with massive replacement chert. Thin } \\
\text { section is nonferroan dolostone (crystals } 20-200 \mu \mathrm{m} \text { ) } \\
\text { with a cherty zone rich in siliceous sponge spicules } \\
\text { and radiolarians(?). Some dolomite rhombs replaced } \\
\text { by silica. } \\
7.9 \text { kg of rock was processed. } \\
\text { Sample collected by A.G. Harris. }\end{array}$ \\
\hline $\begin{array}{l}84 \\
\text { EMA } \\
\text { (Wolverine } \\
\text { Creek plate; } \\
\text { Rok window) }\end{array}$ & $\begin{array}{l}\text { De Long Mtns. } \\
\text { A-2 } \\
68^{\circ} 00^{\prime} 34.6^{\prime \prime} \\
162^{\circ} 59^{\prime} 49^{\prime \prime}\end{array}$ & $\begin{array}{l}\text { Kladognathus } \mathrm{sp} \text {. indet. } \\
3 \mathrm{M}, 2 \mathrm{Sa} \text {, and } 4 \mathrm{Sb}-\mathrm{Sc} \text { elements } \\
1 \mathrm{~Pb} \text { and } 1 \mathrm{Sb} \text { unassigned elements } \\
53 \text { indet. bar, blade, and platform fragments } \\
6 \text { ichthyoliths } \\
\mathrm{CAI}=3-3.5 \\
\text { [98AD15A; 33420-PC] }\end{array}$ & $\begin{array}{l}\text { Mississippian, but not very } \\
\text { earliest Mississippian } \\
\text { (middle Kinderhookian- } \\
\text { Chesterian). }\end{array}$ & $\begin{array}{l}\text { Indeterminate (too few } \\
\text { conodonts). } \\
\text { Conodonts present } \\
\text { suggest shallow-water } \\
\text { depositional setting. }\end{array}$ & $\begin{array}{l}\text { Sample from Kogruk Formation, stratigraphically } \\
\text { above Utukok Formation sample 98AD14G (loc. 83, } \\
\text { table 7). } \\
\text { Six-meter-thick section (sample near base) of light- } \\
\text { gray-weathering, grayish-black, fetid limestone } \\
\text { (mudstone to wackestone to packstone with crinoids, } \\
\text { peloids?), in beds } 5 \mathrm{~cm} \text { thick containing rare chert } \\
\text { nodules. Thin section is skeletal packstone; bioclasts } \\
\text { are mainly crinoid ossicles and lesser foraminifers, } \\
\text { bryozoan fragments, spicules and algae(?). Matrix } \\
\text { contains } 20 \mu \mathrm{m} \text { dolomite rhombs; silica partly } \\
\text { replaces some rhombs and bioclasts. } \\
\text { Heavy-mineral concentrate includes phosphatized } \\
\text { lithic fragments and minor talc-bearing metamorphic } \\
\text { rock fragments. } \\
9.1 \text { kg of rock was processed. }\end{array}$ \\
\hline $\begin{array}{l}87 \\
\text { EMA } \\
\text { (Wolverine } \\
\text { Creek plate; } \\
\text { Rok window) }\end{array}$ & $\begin{array}{l}\text { De Long Mtns. } \\
\text { A-2 } \\
68^{\circ} 01^{\prime} 20^{\prime \prime} \\
162^{\circ} 46^{\prime} 25^{\prime \prime}\end{array}$ & $\begin{array}{l}1 \text { Sa element of Hindeodus sp. indet } \\
\text { CAI }=\sim 3 \text { or } 3.5 \\
\text { [98AKD13] }\end{array}$ & $\begin{array}{l}\text { No older than early Early } \\
\text { Mississippian (middle } \\
\text { Kinderhookian) and no } \\
\text { younger than very earliest } \\
\text { Early Triassic. }\end{array}$ & $\begin{array}{l}\text { Indeterminate (too few } \\
\text { conodonts). }\end{array}$ & $\begin{array}{l}\text { Sample from Kogruk Formation overlying Kayak } \\
\text { Shale on Anxiety Ridge; sample from base of } 50 \text {-ft- } \\
\text { thick Kogruk outcrop. } \\
\text { Medium-gray, fine- to medium-crystalline dolostone } \\
\text { with rare bioclasts and (or) peloids; } 30 \% \text { black chert. } \\
\text { Thin section is dolostone with sparse relict fossils, } \\
\text { including crinoid ossicles to } 2 \text { mm with silicified } \\
\text { centers. Dolomite crystals mostly subhedral, } 300- \\
400 \mu \text { m, with locally abundant intercrystalline } \\
\text { organic material. } \\
7.7 \text { kg of rock was processed. }\end{array}$ \\
\hline
\end{tabular}


Table 6. Conodont samples from the Kogruk Formation-Continued.

[All faunas identified by A.G. Harris. Structural units determined by L.E. Young. EMA, Endicott Mountains allochthon; KRA, Kelly River allochthon. CAI, conodont color alteration index. Letters in field number refer to collector: AD, J.A. Dumoulin; AK, J.S. Kelley; AKD, K.D. Kelley; JS, J.M. Schmidt; KE, K. Evans; KS, K.W. Sherwood; MD, C.F. Mayfield; and TR, I.L. Tailleur. DDH, diamond drill hole. Interval thicknesses in drill holes are drilled thicknesses and have not been corrected for locality]

\begin{tabular}{|c|c|c|c|c|c|}
\hline $\begin{array}{l}\text { Locality No., } \\
\text { structural unit }\end{array}$ & $\begin{array}{l}\text { Quadrangle, } \\
\text { latitude n./ } \\
\text { longitude w. }\end{array}$ & $\begin{array}{c}\text { Conodont fauna and CAI } \\
\text { [field No.; USGS collection No.] }\end{array}$ & Age & \begin{tabular}{|c|} 
Conodont biofacies and \\
depositional \\
environment
\end{tabular} & Remarks \\
\hline $\begin{array}{l}93 \\
\text { KRA(?) } \\
\text { (?Amphitheatre } \\
\text { plate) }\end{array}$ & $\begin{array}{l}\text { DeLong Mtns. } \\
\text { B-1 } \\
68^{\circ} 25^{\prime} 42^{\prime \prime} \\
162^{\circ} 15^{\prime} 50.4^{\prime \prime}\end{array}$ & $\begin{array}{l}9 \text { juvenile Pa elements Gnathodus cf. Gn. defectus } \\
\text { Dunn } \\
2 \text { Pa elements Rhachistognathus muricatus (Dunn) } \\
29 \text { bar, blade, and platform fragments } \\
\text { CAI=3.5-4 } \\
\text { [04AD22A; 33783-PC] }\end{array}$ & $\begin{array}{l}\text { late Late Mississippian (late } \\
\text { Chesterian; Rh. muricatus } \\
\text { Zone through Rh. primus } \\
\text { Zone). }\end{array}$ & $\begin{array}{l}\text { Indeterminate (too few } \\
\text { conodonts); conodonts } \\
\text { present suggest } \\
\text { derivation from a } \\
\text { relatively shallow- } \\
\text { water depositional } \\
\text { setting. }\end{array}$ & $\begin{array}{l}\text { Upper Kogruk Formation(?); highest Lisburne Group } \\
\text { exposed. On trend with Lisburne at localities } 94 \text { and } \\
116 \text { (this table), but black shale and phosphate that } \\
\text { makes up the uppermost Lisburne at these localities } \\
\text { not seen here. Instead, contact zone consists of } \sim 2 \mathrm{~m} \\
\text { of Kogruk talus, overlain by } 5 \text { m of grass, overlain } \\
\text { by rubble of Etivluk Group (olive-brown to } \\
\text { greenish-gray mudstone overlain by green chert). } \\
\text { See text for further discussion of this sample. } \\
\text { Gray-weathering, dark-gray, mildly fetid, quite sooty } \\
\text { limestone. Sample from } 15 \text {-cm-thick interval with 3- } \\
\text { to 5-cm-thick nodular beds. Skeletal mudstone to } \\
\text { wackestone(?), with abundant fossils on bedding } \\
\text { planes; bioclasts include brachiopods to } 5 \text { cm in } \\
\text { diameter, articulated crinoid columnals to } 10 \text { cm } \\
\text { long, and silicified rugose corals. Thin section is } \\
\text { skeletal supportstone that consists of large crinoid, } \\
\text { bryozoan, and brachiopod fragments in a fine- } \\
\text { grained matrix of peloids, small bioclasts (including } \\
\text { foraminifers), calcite cement, and scattered dolomite } \\
\text { rhombs. Some bioclasts partly silicified and (or) } \\
\text { dolomitized. } \\
\text { Heavy-mineral concentrate includes lesser phosphatic } \\
\text { carapace and brachiopod shell fragments. } \\
6.0 \text { kg of rock was processed. }\end{array}$ \\
\hline
\end{tabular}


Table 6. Conodont samples from the Kogruk Formation-Continued.

[All faunas identified by A.G. Harris. Structural units determined by L.E. Young. EMA, Endicott Mountains allochthon; KRA, Kelly River allochthon. CAI, conodont color alteration index. Letters in field number refer to collector: AD, J.A. Dumoulin; AK, J.S. Kelley; AKD, K.D. Kelley; JS, J.M. Schmidt; KE, K. Evans; KS, K.W. Sherwood; MD, C.F. Mayfield; and TR, I.L. Tailleur. DDH, diamond drill hole. Interval thicknesses in drill holes are drilled thicknesses and have not been corrected for locality]

\begin{tabular}{|c|c|c|c|c|c|}
\hline $\begin{array}{l}\text { Locality No., } \\
\text { structural unit }\end{array}$ & $\begin{array}{l}\text { Quadrangle, } \\
\text { latitude n./ } \\
\text { longitude w. }\end{array}$ & $\begin{array}{c}\text { Conodont fauna and CAI } \\
\text { [field No.; USGS collection No.] }\end{array}$ & Age & $\begin{array}{c}\text { Conodont biofacies and } \\
\text { depositional } \\
\text { environment }\end{array}$ & Remarks \\
\hline $\begin{array}{l}94 \\
\text { KRA } \\
\text { (Amphitheatre } \\
\text { plate) }\end{array}$ & $\begin{array}{l}\text { De Long Mtns. } \\
\text { B-1 } \\
68^{\circ} 23^{\prime} 39.5^{\prime \prime} / \\
162^{\circ} 27^{\prime} 21^{\prime \prime}\end{array}$ & $\begin{array}{l}\text { Barren } \\
\text { [03AD27B] }\end{array}$ & & & $\begin{array}{l}\text { Upper Lisburne Group, } 2.5 \mathrm{~m} \text { below top of unit. } \\
\text { Uppermost Lisburne is black shale capped by a layer } \\
\text { of phosphate pebbles to } 14 \mathrm{~cm} \text { in diameter. Basal } \\
\text { Siksikpuk Formation is orange-weathering, greenish } \\
\text { mudstone, with a thin zone of distinctly yellow- } \\
\text { orange-stained clay at the contact. } \\
\text { Sample from top of } 1 \text {-m-thick interval of light-gray- } \\
\text { weathering phosphatic limestone, in } 10 \text { to } 30 \mathrm{~cm} \\
\text { thick beds, underlain and overlain by several m of } \\
\text { carbonaceous black shale. Fresh surface of limestone } \\
\text { has "salt and pepper" look, with abundant black fine- } \\
\text { to coarse-sand-sized phosphate peloids and ooids, as } \\
\text { well as crinoid ossicles and brachiopods. Possible } \\
\text { cm-scale burrows; some filled with phosphate } \\
\text { pellets, others with chert. Thin section is } \\
\text { pelmatozoan-brachiopod-phosphatic clast } \\
\text { grainstone; some phosphatic clasts are ooids. } \\
\text { Heavy-mineral concentrate is mainly variably pyritic } \\
\text { black to dark-gray phosphatized spheroidal grains, } \\
\text { rare phosphatized pelmatozoan ossicles, and very } \\
\text { rare phosphatized gastropod steinkerns. } \\
8.6 \text { kg of rock was processed. } \\
\text { Loc. } 15 \text { (app. 1) of Dumoulin and others (2004). }\end{array}$ \\
\hline
\end{tabular}


Table 6. Conodont samples from the Kogruk Formation-Continued.

[All faunas identified by A.G. Harris. Structural units determined by L.E. Young. EMA, Endicott Mountains allochthon; KRA, Kelly River allochthon. CAI, conodont color alteration index. Letters in field number refer to collector: AD, J.A. Dumoulin; AK, J.S. Kelley; AKD, K.D. Kelley; JS, J.M. Schmidt; KE, K. Evans; KS, K.W. Sherwood; MD, C.F. Mayfield; and TR, I.L. Tailleur. DDH, diamond drill hole. Interval thicknesses in drill holes are drilled thicknesses and have not been corrected for are field descriptions unless otherwise indicated; thin section observations by J.A. Dumoulin. No., number; indet., indeterminate; loc. locality]

\begin{tabular}{|c|c|c|c|c|c|}
\hline $\begin{array}{l}\text { Locality No., } \\
\text { structural unit }\end{array}$ & $\begin{array}{l}\text { Quadrangle, } \\
\text { latitude n./ } \\
\text { longitude w. }\end{array}$ & $\begin{array}{c}\text { Conodont fauna and CAI } \\
\text { [field No.; USGS collection No.] }\end{array}$ & Age & $\begin{array}{c}\text { Conodont biofacies and } \\
\text { depositional } \\
\text { environment } \\
\end{array}$ & Remarks \\
\hline $\begin{array}{l}94 \\
\text { KRA } \\
\text { (Amphitheatre } \\
\text { plate) } \\
\text { [cont.] }\end{array}$ & $\begin{array}{l}\text { De Long Mtns. } \\
\text { B-1 } \\
68^{\circ} 23^{\prime} 39.5^{\prime \prime} / \\
162^{\circ} 27^{\prime} 21^{\prime \prime}\end{array}$ & $\begin{array}{l}5 \text { incomplete Pa elements Gnathodus texanus } \\
\text { Roundy } \\
1 \text { Sa element fragment Kladognathus sp. indet. } \\
28 \text { Pa elements Rhachistognathus prolixus } \\
\text { Baesemann and Lane } \\
1 \text { subadult incomplete Rhachistognathus sp. indet. } \\
\text { Unassigned elements: } \\
1 \mathrm{~Pb} \text { and } 3 \mathrm{M} \\
140 \text { indet. bar, blade, and platform fragments } \\
1 \text { conodont or bryozoan pearl } \\
\text { CAI=2.0-2.5 } \\
\text { [03AD27F; 33758-PC] }\end{array}$ & $\begin{array}{l}\text { middle Late Mississippian } \\
\text { (early Chesterian). } \\
\text { The co-occurrence of Gn. } \\
\text { texanus and Rh. prolixus, } \\
\text { restricts the age of the } \\
\text { collection to the early } \\
\text { Chesterian. }\end{array}$ & $\begin{array}{l}\text { Indeterminate; too few } \\
\text { conodonts. The most } \\
\text { common conodonts in } \\
\text { the collection are } \\
\text { normal-marine species } \\
\text { that occur in platform } \\
\text { to deep-water } \\
\text { depositional settings. } \\
\text { Rh. prolixus is the } \\
\text { earliest } \\
\text { rhachistognathid; } \\
\text { younger species } \\
\text { typically occupied } \\
\text { high-energy, } \\
\text { relatively shallow- } \\
\text { water depositional } \\
\text { regimes. }\end{array}$ & $\begin{array}{l}\text { Upper Lisburne Group, } \sim 5 \mathrm{~m} \text { below top of unit. } \\
\text { Sample from several-m-thick interval of limestone } \\
\text { with partings of black sooty shale. Beds thin (from } \\
10 \text { to } 1 \mathrm{~cm} \text { ) and fine (limestone less grainy, shaly } \\
\text { partings increase) upward. Lower beds are dark- } \\
\text { gray, medium-gray-weathering skeletal } \\
\text { wackestone/packstone with crinoid ossicles and } \\
\text { productid brachiopods to } 3 \mathrm{~cm} \text {. Upper beds are } \\
\text { crinoid mudstone to wackestone that weathers } \\
\text { medium dark gray; beds wavy and irregular. Both } \\
\text { upper and lower beds included in sample. Thin } \\
\text { section is skeletal packstone/grainstone to mudstone } \\
\text { (mostly wacke/packstone) with dark, noncarbonate } \\
\text { mud matrix and minor dolomite and barite; bioclasts } \\
\text { mostly pelmatozoan fragments, lesser brachiopods, } \\
\text { and ostracodes. } \\
\text { Heavy-mineral concentrate consists mainly of } \\
\text { phosphatized rock fragments, dolomitized } \\
\text { pelmatozoan ossicles, lesser phosphatized gastropod } \\
\text { steinkerns, and scarce phosphatic brachiopod } \\
\text { fragments. } \\
10 \text { kg of rock was processed. }\end{array}$ \\
\hline $\begin{array}{l}99 \\
\text { KRA } \\
\text { (Amphitheatre } \\
\text { plate) }\end{array}$ & $\begin{array}{l}\text { De Long Mtns. } \\
\text { B-1 } \\
68^{\circ} 19^{\prime} 22^{\prime \prime} / \\
162^{\circ} 20^{\prime} 10^{\prime \prime}\end{array}$ & $\begin{array}{l}1 \text { gnathodid } \mathrm{M} \text { element } \\
\text { Unassigned elements: } \\
3 \text { juvenile } \mathrm{Pa}(2 \text { morphotypes) and } 1 \mathrm{Sa} \\
38 \text { indet. bar, blade, and platform fragments } \\
\mathrm{CAI}=2.5 \\
{[00 \mathrm{AD} 10 \mathrm{~A} ; 33629-\mathrm{PC}]}\end{array}$ & Mississippian. & $\begin{array}{l}\text { Indeterminate (too few } \\
\text { conodonts). }\end{array}$ & $\begin{array}{l}\text { About } 30 \mathrm{~m} \text { above base of Kogruk Formation, which } \\
\text { here overlies the micritic limestone unit of Curtis } \\
\text { and others (1990); see table } 8 \text { for a sample from the } \\
\text { micritic limestone unit at this locality. } \\
\text { Light-gray- to medium-light-gray-weathering, } \\
\text { medium-dark-gray lime mudstone/wackestone with } \\
\text { 10\% brown to black chert (probably replacing } \\
\text { bioclastic intervals); beds } 10 \text { to } 30 \mathrm{~cm} \text { thick. Thin } \\
\text { section is dolomitic calcareous spiculite with rare } \\
\text { crinoid ossicles. } \\
\text { Heavy-mineral concentrate includes fragments of } \\
\text { phosphatic chert and rare phosphatic brachiopod } \\
\text { fragments. } \\
11.52 \mathrm{~kg} \text { of rock was processed. } \\
\text { Loc. } 14 \text { (app. 1) of Dumoulin and others (2004). }\end{array}$ \\
\hline
\end{tabular}


Table 6. Conodont samples from the Kogruk Formation-Continued.

[All faunas identified by A.G. Harris. Structural units determined by L.E. Young. EMA, Endicott Mountains allochthon; KRA, Kelly River allochthon. CAI, conodont color alteration index. Letters in field number refer to collector: AD, J.A. Dumoulin; AK, J.S. Kelley; AKD, K.D. Kelley; JS, J.M. Schmidt; KE, K. Evans; KS, K.W. Sherwood; MD, C.F. Mayfield; and TR, I.L. Tailleur. DDH, diamond drill hole. Interval thicknesses in drill holes are drilled thicknesses and have not been corrected for are field descriptions unless otherwise indicated; thin section observations by J.A. Dumoulin. No., number; indet., indeterminate; loc., locality]

\begin{tabular}{|c|c|c|c|c|c|}
\hline $\begin{array}{l}\text { Locality No., } \\
\text { structural unit }\end{array}$ & $\begin{array}{l}\text { Quadrangle, } \\
\text { latitude } \mathrm{n} . / \\
\text { longitude w. }\end{array}$ & $\begin{array}{c}\text { Conodont fauna and CAI } \\
\text { [field No.; USGS collection No.] }\end{array}$ & Age & $\begin{array}{c}\text { Conodont biofacies and } \\
\text { depositional } \\
\text { environment } \\
\end{array}$ & Remarks \\
\hline \multirow[t]{2}{*}{$\begin{array}{l}110 \\
\text { KRA (Kelly } \\
\text { plate) }\end{array}$} & \multirow[t]{2}{*}{$\begin{array}{l}\text { De Long Mtns. } \\
\text { A-1 } \\
68^{\circ} 14^{\prime} 01.7^{\prime \prime \prime} / \\
162^{\circ} 05^{\prime} 10.8^{\prime \prime}\end{array}$} & $\begin{array}{l}\text { Kladognathus sp. indet. } \\
1 \mathrm{M} \text { and } 2 \mathrm{Sb}-\mathrm{Sc} \text { elements } \\
1 \text { unassigned } \mathrm{Pb} \text { element } \\
46 \text { small indet. bar, blade, and platform fragments } \\
\mathrm{CAI}=2.0-2.5 \\
{[03 \mathrm{AD} 29 \mathrm{~A} ; 33763-\mathrm{PC}]}\end{array}$ & $\begin{array}{l}\text { Mississippian; the only } \\
\text { generically identifiable } \\
\text { conodont in this collection } \\
\text { is Kladognathus, which } \\
\text { ranges from within the } \\
\text { Kinderhookian through } \\
\text { Chesterian. }\end{array}$ & $\begin{array}{l}\text { Indeterminate (too few } \\
\text { generically } \\
\text { determinate } \\
\text { conodonts); the } \\
\text { relatively common, } \\
\text { small indeterminate } \\
\text { conodont fragments } \\
\text { indicate a postmortem } \\
\text { hydraulic winnow. }\end{array}$ & $\begin{array}{l}\text { Sample } \sim 10-15 \mathrm{~m} \text { stratigraphically below top of } \\
\text { Kogruk Formation, which is here structurally } \\
\text { overlain by ultramafic rocks of the Misheguk } \\
\text { Mountain allochthon; contact probably a normal } \\
\text { fault. } \\
\text { Light- to medium-brownish-gray limestone, weathers } \\
\text { light to very light gray. Skeletal wackestone with } \\
\text { local colonial and solitary corals; } 30 \text {-cm-thick } \\
\text { interval with } 10 \text { - to } 15-\mathrm{cm} \text {-thick beds. Thin section is } \\
\text { fractured and deformed crinoid grainstone with } \\
\text { bryozoans, brachiopods, and rare foraminifers. } \\
6.4 \text { kg of rock was processed. } \\
\end{array}$ \\
\hline & & $\begin{array}{l}\text { Kladognathus sp. } \\
1 \mathrm{Sa} \text { and } 1 \mathrm{Sb}-\mathrm{Sc} \text { element fragments } \\
5 \mathrm{~S} \text { elements Synclydognathus geminus (Hinde) } \\
\text { Unassigned elements: } \\
2 \mathrm{~Pa} \text { and } 1 \mathrm{Sc} \\
48 \text { rather small indet. bar, blade, and platform } \\
\text { fragments } \\
\text { CAI=2.0-2.5 } \\
\text { [03AD29B; 33761-PC] }\end{array}$ & $\begin{array}{l}\text { late Early-middle Late } \\
\text { Mississippian (from within } \\
\text { the early Osagean-very } \\
\text { early Chesterian). }\end{array}$ & $\begin{array}{l}\text { Indeterminate (too few } \\
\text { conodonts). Likely a } \\
\text { winnow; all } \\
\text { conodonts are small, } \\
\text { relatively thin element } \\
\text { fragments. }\end{array}$ & $\begin{array}{l}\text { Sample } \sim 2-3 \mathrm{~m} \text { stratigraphically above 03AD29A. } \\
\text { Rubble crop (30-cm-thick beds) of medium-brownish- } \\
\text { gray limestone, weathers light gray. Skeletal } \\
\text { packstone with crinoid ossicles, silicified rugose } \\
\text { corals, and peloids(?). Thin section is partly } \\
\text { dolomitized crinoid supportstone with rare coral } \\
\text { fragments and patchy chert replacement. } \\
6.4 \text { kg of rock was processed. }\end{array}$ \\
\hline $\begin{array}{l}111 \\
\text { KRA }\end{array}$ & $\begin{array}{l}\text { De Long Mtns. } \\
\text { A-1 } \\
68^{\circ} 11^{\prime} 41.8^{\prime \prime} / \\
162^{\circ} 27^{\prime} 22^{\prime \prime}\end{array}$ & $\begin{array}{l}10 \text { deformed and sheared Pa element fragments } \\
\text { Bispathodus sp. indet. [either Bi. stabilis } \\
\text { (Branson and Mehl) or Bi. utahensis Sandberg } \\
\text { and Gutschick)] } \\
39 \text { indet. bar, blade, and platform fragments } \\
\text { CAI=3-3.5 } \\
\text { [97AK23A] }\end{array}$ & $\begin{array}{l}\text { late Late Devonian-middle } \\
\text { Late Mississippian (middle } \\
\text { Famennian-Meramecian). }\end{array}$ & $\begin{array}{l}\text { Single-row bispathodid } \\
\text { biofacies; normal- } \\
\text { marine, shallow- to } \\
\text { deep-water } \\
\text { depositional setting. }\end{array}$ & $\begin{array}{l}\text { Sample from Kogruk Formation in fault contact below } \\
\text { Utukok Formation; see table } 7 \text { for Utukok sample } \\
\text { from this locality. } \\
\text { Medium-light-gray-weathering dolostone with rare } \\
\text { crinoid fragments and irregular dark-gray to black } \\
\text { chert nodules. Thin section is partly dolomitized } \\
\text { skeletal wackestone/packstone with dolomite rhombs } \\
(40-200 \mu \mathrm{m}) \text { and }<1 \% \text { detrital quartz (and feldspar?) } \\
\text { silt and sand. Bioclasts mostly crenulated brachiopod } \\
\text { fragments (to } 1 \mathrm{~cm} \text { ) and crinoid ossicles, with minor } \\
\text { calcispheres. } \\
5.0 \text { kg of rock was processed. }\end{array}$ \\
\hline
\end{tabular}


Table 6. Conodont samples from the Kogruk Formation-Continued.

[All faunas identified by A.G. Harris. Structural units determined by L.E. Young. EMA, Endicott Mountains allochthon; KRA, Kelly River allochthon. CAI, conodont color alteration index. Letters in field number refer to collector: AD, J.A. Dumoulin; AK, J.S. Kelley; AKD, K.D. Kelley; JS, J.M. Schmidt; KE, K. Evans; KS, K.W. Sherwood; MD, C.F. Mayfield; and TR, I.L. Tailleur. DDH, diamond drill hole. Interval thicknesses in drill holes are drilled thicknesses and have not been corrected for locality]

\begin{tabular}{|c|c|c|c|c|c|}
\hline $\begin{array}{l}\text { Locality No., } \\
\text { structural unit }\end{array}$ & $\begin{array}{l}\text { Quadrangle, } \\
\text { latitude n./ } \\
\text { longitude w. }\end{array}$ & $\begin{array}{c}\text { Conodont fauna and CAI } \\
\text { [field No.; USGS collection No.] }\end{array}$ & Age & $\begin{array}{c}\text { Conodont biofacies and } \\
\text { depositional } \\
\text { environment }\end{array}$ & Remarks \\
\hline $\begin{array}{ll}113 \\
\text { KRA }\end{array}$ & $\begin{array}{l}\text { De Long Mtns. } \\
\text { A-1 } \\
68^{\circ} 12^{\prime} 00^{\prime \prime} / \\
162^{\circ} 26^{\prime} 12^{\prime \prime}\end{array}$ & $\begin{array}{l}\text { All conodonts are multiply fractured and all are } \\
\text { incomplete. } \\
1 \text { mid Pa element fragment Hindeodus sp. indet. } \\
1 \text { deformed and fractured incomplete Pa element } \\
\text { Cavusgnathus? sp. indet. } \\
1 \text { Pa posterior tip fragment Cavusgnathus sp. indet. } \\
14 \text { indet. bar, blade, and platform fragments } \\
\text { CAI=3-3.5 } \\
\text { [97AK46A; 33394-PC] }\end{array}$ & $\begin{array}{l}\text { middle-late Late } \\
\text { Mississippian (late } \\
\text { Meramecian-Chesterian). }\end{array}$ & $\begin{array}{l}\text { Indeterminate (too few } \\
\text { generically } \\
\text { identifiable } \\
\text { conodonts). }\end{array}$ & $\begin{array}{l}\text { Samples Kogruk Formation just above contact with } \\
\text { underlying Utukok Formation; see table } 7 \text { for } \\
\text { Utukok sample from this locality. } \\
\text { Light-gray-weathering, medium-gray limestone with } \\
10 \text { to } 20 \% \text { black irregular chert nodules. Thin section } \\
\text { is pervasively dolomitized limestone with abundant } \\
\text { calcite-filled fractures and veins. Dolomite } \sim 80 \% \text { or } \\
\text { more, in euhedral rhombs } 20-200 \mu \mathrm{m} \text { in diameter. A } \\
\text { few remnant crinoid ossicles (to } 1 \mathrm{~mm} \text { diameter) } \\
\text { may still be calcite. } \\
7.0 \mathrm{~kg} \text { of rock was processed. }\end{array}$ \\
\hline \multirow[t]{2}{*}{$\begin{array}{l}114 \\
\text { KRA }\end{array}$} & \multirow[t]{2}{*}{$\begin{array}{l}\text { De Long Mtns. } \\
\text { A-1 } \\
68^{\circ} 12^{\prime} 0.6^{\prime \prime} / \\
162^{\circ} 25^{\prime} 12^{\prime \prime}\end{array}$} & $\begin{array}{l}1 \text { incomplete Pa element Cavusgnathus sp. indet. } \\
1 \text { juvenile Pa element Gnathodus homopunctatus } \\
\text { (Ziegler)? [pl. 2, fig. 13] } \\
7 \text { indet. bar, blade, and platform fragments } \\
\text { CAI=3-3.5 } \\
\text { [97AK51A; 33395-PC] }\end{array}$ & $\begin{array}{l}\text { Late Mississippian (late } \\
\text { Meramecian-early } \\
\text { Chesterian). }\end{array}$ & $\begin{array}{l}\text { Indeterminate (too few } \\
\text { generically } \\
\text { identifiable } \\
\text { conodonts). }\end{array}$ & $\begin{array}{l}\text { Sample taken at top of } \sim 40 \text {-ft-thick outcrop of } \\
\text { limestone and chert overlying } 5 \text {-ft-thick rubble zone } \\
\text { overlying outcrop of Kayak Shale(?). Thin section is } \\
\text { siliceous spiculite with rare radiolarians }(?) \text { and } \\
\text { subordinate dolomite rhombs }(8-25 \mu \mathrm{m}) \text { cross-cut by } \\
\text { fractures and quartz veins. } \\
7.9 \mathrm{~kg} \text { of rock was processed. }\end{array}$ \\
\hline & & $\begin{array}{l}5 \text { Pa element fragments Bispathodus stabilis } \\
\text { (Branson and Mehl) or Bi. utahensis Sandberg } \\
\text { and Gutschick } \\
1 \text { unassigned Pb element } \\
27 \text { indet. bar, blade, and platform fragments } \\
\text { CAI=3 } \\
\text { [97AK51C; 33401-PC] }\end{array}$ & $\begin{array}{l}\text { Mississippian (late } \\
\text { Kinderhookian-early } \\
\text { Chesterian). }\end{array}$ & $\begin{array}{l}\text { Indeterminate (too few } \\
\text { generically } \\
\text { identifiable } \\
\text { conodonts). } \\
\text { Conodonts present } \\
\text { indicate postmortem } \\
\text { transport in a normal- } \\
\text { marine environment. }\end{array}$ & $\begin{array}{l}\text { Sample taken at base of } \sim 40 \text {-ft-thick outcrop of } \\
\text { limestone and chert just above 5-ft-thick rubble zone } \\
\text { overlying outcrop of Kayak Shale(?). Thin section is } \\
\text { calcareous spiculite with minor crinoid ossicles and } \\
\text { thin-shelled, spar-filled ostracodes, a lime mud } \\
\text { matrix and trace feldspar(?). } \\
\text { Heavy-mineral concentrate includes phosphatic } \\
\text { brachiopod fragments. } \\
7.0 \text { kg of rock was processed. }\end{array}$ \\
\hline
\end{tabular}


Table 6. Conodont samples from the Kogruk Formation-Continued.

[All faunas identified by A.G. Harris. Structural units determined by L.E. Young. EMA, Endicott Mountains allochthon; KRA, Kelly River allochthon. CAI, conodont color alteration index. Letters in field number refer to collector: AD, J.A. Dumoulin; AK, J.S. Kelley; AKD, K.D. Kelley; JS, J.M. Schmidt; KE, K. Evans; KS, K.W. Sherwood; MD, C.F. Mayfield; and TR, I.L. Tailleur. DDH, diamond drill hole. Interval thicknesses in drill holes are drilled thicknesses and have not been corrected for 作 locality]

\begin{tabular}{|c|c|c|c|c|c|}
\hline $\begin{array}{l}\text { Locality No., } \\
\text { structural unit }\end{array}$ & $\begin{array}{l}\text { Quadrangle, } \\
\text { latitude n./ } \\
\text { longitude w. }\end{array}$ & $\begin{array}{c}\text { Conodont fauna and CAI } \\
\text { [field No.; USGS collection No.] }\end{array}$ & Age & $\begin{array}{c}\text { Conodont biofacies and } \\
\text { depositional } \\
\text { environment }\end{array}$ & Remarks \\
\hline $\begin{array}{l}116 \\
\text { KRA (Kelly } \\
\text { plate) }\end{array}$ & $\begin{array}{l}\text { Misheguk Mtn. } \\
\text { B-5 } \\
68^{\circ} 27^{\prime} 37.6^{\prime \prime} / \\
161^{\circ} 58^{\prime} 23.4^{\prime \prime}\end{array}$ & $\begin{array}{l}1 \mathrm{~Pa} \text { element fragment Gnathodus texanus Roundy } \\
1 \text { incomplete Pa element Hindeodus sp. } \\
\text { Idioprioniodus sp. } \\
1 \mathrm{~Pb} \text { and } 2 \text { bar fragments } \\
1 \mathrm{Sb}-\mathrm{Sc} \text { element Kladognathus sp. } \\
18 \mathrm{~Pa} \text { elements (mostly juveniles) } \\
\text { Rhachistognathus prolixus } \text { Baesemann and Lane } \\
\text { Unassigned elements: } \\
6 \mathrm{~Pb}(3 \text { morphotypes), } 4 \mathrm{M} \text {, and } 1 \mathrm{Sa} \\
111 \text { indet. bar, blade, and platform fragments } \\
\text { Conodonts are partly to considerably coated with } \\
\text { organic matter so that CAI is approximate but not } \\
\text { greater than } 2.5 \text {. } \\
\text { [03AD38B; } 33762-\mathrm{PC}]\end{array}$ & $\begin{array}{l}\text { middle Late Mississippian } \\
\text { (early Chesterian). } \\
\text { This fauna correlates well } \\
\text { with that of 03AD27F (loc. } \\
\text { 94, this table). }\end{array}$ & $\begin{array}{l}\text { Indeterminate (too few } \\
\text { generically } \\
\text { determinate } \\
\text { conodonts). The } \\
\text { rhachistognathids } \\
\text { represent post-mortem } \\
\text { transport (a winnow) } \\
\text { from a shallow-water } \\
\text { setting; the relative } \\
\text { abundance of very } \\
\text { small indeterminate } \\
\text { bar, blade, and } \\
\text { platform fragments } \\
\text { reinforces the } \\
\text { interpretation of a } \\
\text { winnow. }\end{array}$ & $\begin{array}{l}\text { Uppermost Lisburne Group. Sample from a } 50 \text {-cm- } \\
\text { thick interval of limestone overlain by } \sim 1 \mathrm{~m} \text { of black } \\
\text { shale capped by } \sim 5-10 \mathrm{~cm} \text { of limy phosphatic rubble. } \\
\text { The phosphate is overlain by } \sim 10 \text { m of cover, and } \\
\text { then orange-weathering, olive-gray siliceous } \\
\text { mudstone of the Etivluk Group. } \\
\text { Dark-gray to dark-brownish-gray limestone, weathers } \\
\text { medium light gray, in } 5 \text { - to } 10-\mathrm{cm} \text {-thick beds; } \\
\text { crinoid-brachiopod wacke/packstone with bioclasts } \\
\text { (some silicified) to } 2 \mathrm{~cm} \text { long. Thin section is } \\
\text { brachiopod packstone with crinoid ossicles and } \\
\text { ostracodes, minor quartz silt and dolomite rhombs, } \\
\text { and rare phosphatic(?) clasts. } \\
\text { Heavy-mineral concentrate (nonmagnetic fraction) is } \\
\text { chiefly phosphatic and phosphatized mudstone to } \\
\text { siltstone fragments with minor phosphatic } \\
\text { brachiopod fragments and phosphatic tubes or tube } \\
\text { linings and rare composite glauconite-phosphate- } \\
\text { rhombohedral dolomite grains. } \\
3.0 \text { kg of rock was processed. } \\
\text { Loc. } 17 \text { (app. 1) of Dumoulin and others (2004). }\end{array}$ \\
\hline $\begin{array}{l}121 \\
\text { EMA (Key } \\
\text { Creek plate) }\end{array}$ & $\begin{array}{l}\text { Misheguk Mtn. } \\
\text { A-4 } \\
68^{\circ} 14^{\prime} 35^{\prime \prime} / \\
161^{\circ} 20^{\prime} 16^{\prime \prime}\end{array}$ & $\begin{array}{l}\text { All conodonts are small, mechanically broken } \\
\text { fragments. Although they have relatively low } \\
\text { thermal maturity, the conodonts have undergone } \\
\text { substantial brittle deformation. } \\
1 \text { Pa element fragment of a cavusgnathoid } \\
2 \text { juvenile incomplete Pa elements Gnathodus sp. } \\
\text { indet. } \\
1 \text { Pa element fragment Hindeodus sp. indet. } \\
1 \text { Sb-Sc element fragment Kladognathus } \text { sp. indet. } \\
\text { Synclydognathus sp. indet. } \\
2 \mathrm{M} \text { and (or) S element fragments } \\
\text { Unassigned elements: } \\
1 \mathrm{M} \text { and } 1 \text { Sa (fragments) } \\
134 \text { indet. bar, blade, and platform fragments } \\
\text { CAI=3 } \\
\text { [00AD28A; 33639-PC] }\end{array}$ & $\begin{array}{l}\text { early-middle Late } \\
\text { Mississippian } \\
\text { (Meramecian-early } \\
\text { Chesterian). }\end{array}$ & $\begin{array}{l}\text { Indeterminate (too few } \\
\text { generically } \\
\text { determinate } \\
\text { conodonts). Species } \\
\text { association indicates a } \\
\text { probable inner to } \\
\text { middle shelf } \\
\text { depositional setting }\end{array}$ & $\begin{array}{l}\text { Kogruk and Kuna Formations appear to interfinger in } \\
\text { this area. Sample from 8- to 10-m-high massive } \\
\text { outcrop of Kogruk. } \\
\text { Medium-gray to medium-dark-gray limestone, } \\
\text { weathers mottled light to medium light gray. Thin- } \\
\text { section is partly dolomitized crinoidal supportstone } \\
\text { with abundant veins of sparry calcite. } \\
\text { Heavy-mineral concentrate includes phosphatized rock } \\
\text { fragments and lesser indeterminate phosphatized } \\
\text { bioclasts. } \\
9.85 \mathrm{~kg} \text { of rock was processed. } \\
\text { Loc. } 11 \text { (app. 1) of Dumoulin and others (2004). }\end{array}$ \\
\hline
\end{tabular}


Table 6. Conodont samples from the Kogruk Formation-Continued.

[All faunas identified by A.G. Harris. Structural units determined by L.E. Young. EMA, Endicott Mountains allochthon; KRA, Kelly River allochthon. CAI, conodont color alteration index. Letters in field number refer to collector: AD, J.A. Dumoulin; AK, J.S. Kelley; AKD, K.D. Kelley; JS, J.M. Schmidt; KE, K. Evans; KS, K.W. Sherwood; MD, C.F. Mayfield; and TR, I.L. Tailleur. DDH, diamond drill hole. Interval thicknesses in drill holes are drilled thicknesses and have not been corrected for

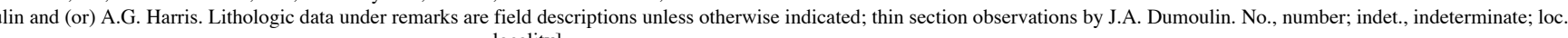
locality]

\begin{tabular}{|c|c|c|c|c|c|}
\hline $\begin{array}{l}\text { Locality No., } \\
\text { structural unit }\end{array}$ & $\begin{array}{l}\text { Quadrangle, } \\
\text { latitude n./ } \\
\text { longitude w. }\end{array}$ & $\begin{array}{c}\text { Conodont fauna and CAI } \\
\text { [field No.; USGS collection No.] }\end{array}$ & Age & $\begin{array}{c}\text { Conodont biofacies and } \\
\text { depositional } \\
\text { environment }\end{array}$ & Remarks \\
\hline $\begin{array}{l}147 \\
\text { Olistolith } \\
\text { derived from } \\
\text { EMA } \\
\text { (Wolverine } \\
\text { Creek plate) }\end{array}$ & $\begin{array}{l}\text { De Long Mtns. } \\
\text { A-2 } \\
68^{\circ} 04^{\prime} 30^{\prime \prime} / \\
162^{\circ} 50^{\prime} 12.5^{\prime \prime}\end{array}$ & $\begin{array}{l}6 \text { Pa element fragments Hindeodus crassidentatus } \\
\text { (Branson and Mehl)? } \\
26 \text { indet. bar, blade, and platform fragments } \\
\\
\text { CAI=2-2.5 } \\
\text { [DDH 550 (Aqqaluk deposit), composite conodont } \\
\text { sample: } 273.5-274.5,277.5-278.5,287-288 \mathrm{ft} ; \\
\text { 33619-PC] }\end{array}$ & \begin{tabular}{|l|} 
Early, but not earliest, \\
Mississippian (middle \\
Kinderhookian-Osagean).
\end{tabular} & $\begin{array}{l}\text { Indeterminate (too few } \\
\text { identifiable } \\
\text { conodonts); conodonts } \\
\text { present indicate } \\
\text { postmortem transport } \\
\text { within or from a high- } \\
\text { energy, relatively } \\
\text { shallow-water } \\
\text { depositional setting. } \\
\end{array}$ & $\begin{array}{l}\text { Kogruk Formation(?) near base of drill hole. } \\
273.5 \text {-288.8 ft: Light- to medium-gray, bioclastic, } \\
\text { dolomitized, carbonate rock. } \\
\text { Thin-section data: } \\
287 \mathrm{ft} \text { : Brecciated dolostone with relict bioclasts } \\
\text { (crinoid ossicles). } \\
6.3 \mathrm{~kg} \text { of rock was processed. }\end{array}$ \\
\hline $\begin{array}{l}148 \\
\text { Olistolith } \\
\text { derived from } \\
\text { EMA } \\
\text { (Wolverine } \\
\text { Creek plate) }\end{array}$ & $\begin{array}{l}\text { De Long Mtns. } \\
\text { A-2 } \\
68^{\circ} 04^{\prime} 28.6^{\prime \prime} / \\
162^{\circ} 50^{\prime} 04.3^{\prime \prime}\end{array}$ & $\begin{array}{l}7 \text { Pa elements (all incomplete) Bispathodus } \\
\text { utahensis Sandberg and Gutschick? } \\
\text { Hindeodus crassidentatus (Branson and Mehl) } \\
3 \mathrm{~Pa} \text { (all incomplete) and } 1 \mathrm{~Pb} \text { elements } \\
\text { 1 Sb-Sc element Kladognathus sp. indet. } \\
\text { Unassigned elements: } \\
1 \mathrm{M}, 1 \mathrm{Sa} \text {, } 1 \mathrm{Sb} \text {, and } 7 \mathrm{Sc} \text { (3 morphotypes) } \\
117 \text { indet. bar, blade, and platform fragments } \\
\text { CAI=2 } \\
\text { [DDH } 605 \text { (Main deposit), composite conodont } \\
\text { sample: } 350-351,354-355.5,356-357,357.5- \\
358.5,359-360.3,365.3-367 \mathrm{ft} ; 33620-\mathrm{PC}]\end{array}$ & \begin{tabular}{|l|} 
Early, but not earliest, \\
Mississippian (late \\
Kinderhookian-Osagean).
\end{tabular} & $\begin{array}{l}\text { Indeterminate (too few } \\
\text { identifiable } \\
\text { conodonts); conodonts } \\
\text { present indicate } \\
\text { postmortem transport } \\
\text { within or from a high- } \\
\text { energy, relatively } \\
\text { shallow-water } \\
\text { depositional setting. }\end{array}$ & $\begin{array}{l}\text { Kogruk Formation(?) (middle? part of unit). } \\
\text { 350-367 ft: Calcareous dolostone to dolomitic } \\
\text { limestone; medium-gray to medium-light-gray, } \\
\text { variably brecciated. } \\
\text { Thin-section data: } \\
\text { 350, } 362.8 \mathrm{ft}: \text { Brecciated dolostone with relict bioclasts } \\
\text { (crinoid ossicles). } \\
\text { Heavy-mineral concentrate contains composite } \\
\text { dolomite-bearing phosphatized rock fragments and } \\
\text { indeterminate bioclasts. } \\
9.54 \mathrm{~kg} \text { of rock was processed. }\end{array}$ \\
\hline $\begin{array}{l}159 \\
\text { EMA (Key } \\
\text { Creek plate; } \\
\text { Port Road } \\
\text { succession of } \\
\text { Dumoulin and } \\
\text { others, 2004) }\end{array}$ & $\begin{array}{l}\text { Noatak D-3 } \\
67^{\circ} 51^{\prime} 04^{\prime \prime} / \\
163^{\circ} 15^{\prime} 25^{\prime \prime} \\
\end{array}$ & $\begin{array}{l}1 \text { incomplete Pa element Bispathodus utahensis } \\
\text { Sandberg and Gutschick } \\
1 \text { incomplete Pa element Hindeodus sp. indet. } \\
\text { Kladognathus sp. indet. } \\
6 \mathrm{M} \text { and } 5 \mathrm{Sb}-\mathrm{Sc} \text { elements } \\
1 \text { unassigned ozarkodinid Pb element } \\
1 \text { Sc element Synclydognathus geminus (Hinde) } \\
68 \text { indet. bar, blade, and platform fragments } \\
\\
\text { CAI=2.5 } \\
\text { [DDH 1103, composite conodont Sample 1: 643.2- } \\
\text { 43.8, 644-44.5, 645.5-46, 648.5-49, 658-58.5, } \\
\text { 660-60.5, 662-62.5, 663-63.5, 667-67.3 ft; } \\
\text { 33658-PC] }\end{array}$ & $\begin{array}{l}\text { late Early-middle Late } \\
\text { Mississippian (Osagean- } \\
\text { early Chesterian). }\end{array}$ & $\begin{array}{l}\text { Indeterminate (too few } \\
\text { conodonts); conodonts } \\
\text { that are present all } \\
\text { indicate postmortem } \\
\text { transport within or } \\
\text { from a relatively high- } \\
\text { energy, shallow-water } \\
\text { depositional } \\
\text { environment. }\end{array}$ & $\begin{array}{l}\text { Lowest Kogruk Formation, just above gradational } \\
\text { contact with Utukok Formation at } \sim 668 \mathrm{ft} \text {. See tables } \\
7 \text { and } 11 \mathrm{for} \text { additional samples from this drill hole. } \\
643.2-667.3 \mathrm{ft} \text { : White to light-gray, bioclastic } \\
\text { (crinoidal), dolomitized carbonate with } 15-30 \% \\
\text { light- to medium-gray chert nodules and interbeds as } \\
\text { much as } 2.5 \mathrm{ft} \text { thick of green noncalcareous } \\
\text { mudstone (chert and mudstone not sampled for } \\
\text { conodonts). } \\
\text { Thin section data: } \\
643.8 \mathrm{ft} \text { : Brecciated dolostone with patchy chert. } \\
665 \mathrm{ft} \text { Coarse crystalline dolostone with patchy chert, } \\
\text { relict crinoid ossicles to } 4 \text { mm in diameter, and rare } \\
\text { bryozoan fragments. } \\
\text { Heavy-mineral concentrate includes scarce coralline } \\
\text { fragments. } \\
6.3 \text { kg of rock was processed. } \\
\text { Loc. } 8 \text { (app. 1) of Dumoulin and others (2004). }\end{array}$ \\
\hline
\end{tabular}


Table 6. Conodont samples from the Kogruk Formation-Continued.

[All faunas identified by A.G. Harris. Structural units determined by L.E. Young. EMA, Endicott Mountains allochthon; KRA, Kelly River allochthon. CAI, conodont color alteration index. Letters in field number refer to collector: AD, J.A. Dumoulin; AK, J.S. Kelley; AKD, K.D. Kelley; JS, J.M. Schmidt; KE, K. Evans; KS, K.W. Sherwood; MD, C.F. Mayfield; and TR, I.L. Tailleur. DDH, diamond drill hole. Interval thicknesses in drill holes are drilled thicknesses and have not been corrected for locality]

\begin{tabular}{|c|c|c|c|c|c|}
\hline $\begin{array}{l}\text { Locality No., } \\
\text { structural unit }\end{array}$ & $\begin{array}{l}\text { Quadrangle, } \\
\text { latitude n./ } \\
\text { longitude w. }\end{array}$ & $\begin{array}{c}\text { Conodont fauna and CAI } \\
\text { [field No.; USGS collection No.] }\end{array}$ & Age & $\begin{array}{c}\text { Conodont biofacies and } \\
\text { depositional } \\
\text { environment }\end{array}$ & Remarks \\
\hline $\begin{array}{l}160 \\
\text { EMA (Key } \\
\text { Creek plate; } \\
\text { Port Road } \\
\text { succession of } \\
\text { Dumoulin and } \\
\text { others, 2004) }\end{array}$ & $\begin{array}{l}\text { Noatak D-3 } \\
67^{\circ} 52^{\prime} 40^{\prime \prime} / \\
163^{\circ} 11^{\prime \prime} 00^{\prime \prime}\end{array}$ & $\begin{array}{l}\text { Bispathodus utahensis Sandberg and Gutschick } \\
71 \mathrm{~Pa}, 1 \mathrm{~Pb}, 3 \mathrm{M}, 1 \mathrm{Sb} \text { and } 1 \mathrm{Sc} \text { elements } \\
1 \mathrm{P} \text { element fragment Embaysgnathus? sp. } \\
\text { Idioprioniodus spp. indet. } \\
2 \mathrm{~Pa}, 2 \mathrm{~Pb}, 5 \mathrm{M} \text { (mostly incomplete), and } 4 \mathrm{~S} \\
\text { elements } \\
\text { Kladognathus tenuis (Branson and Mehl) } \\
1 \mathrm{~Pa}-\mathrm{Pb}, 19 \mathrm{M}, 10 \mathrm{Sa}, 38 \mathrm{Sb}-\mathrm{Sc} \text {, and } 23 \mathrm{bar} \\
\text { fragments (all conodonts are incomplete) } \\
285 \text { indet. bar, blade, and platform fragments } \\
\text { CAI=3 } \\
\text { [DDH 1104, composite conodont sample } 1: 678-80 \text {, } \\
695-95.5,699-701,703-04,709-10,711-11.5 \mathrm{ft} \text {; } \\
\text { 33662-PC] }\end{array}$ & $\begin{array}{l}\text { latest Early-earliest Late } \\
\text { Mississippian (late } \\
\text { Osagean-early Meramecian; } \\
\text { Po. mehli-Lower Gn. } \\
\text { texanus Zone to Gn. } \\
\text { homopunctatus-Upper Gn. } \\
\text { texanus Zone, possibly Gn. } \\
\text { homopunctatus-Upper Gn. } \\
\text { texanus Zone). }\end{array}$ & $\begin{array}{l}\text { Post-mortem transport } \\
\text { within or from a } \\
\text { bispathodid- } \\
\text { kladognathid } \\
\text { biofacies. } \\
\text { Taphonomy suggests } \\
\text { a relatively shallow- } \\
\text { water depositional } \\
\text { setting. }\end{array}$ & $\begin{array}{l}\text { Upper Kogruk Formation, here overlain by Ikalukrok } \\
\text { unit of Kuna Formation; contact (at } 670 \mathrm{ft} \text { ) could be } \\
\text { a fault. } \\
678-711.5 \mathrm{ft} \text { : Very light to light-gray (outer), medium- } \\
\text { light to light-gray (inner), locally vuggy, locally } \\
\text { mottled dolostone with } 15-20 \% \text { medium-dark-gray } \\
\text { (outer), brownish-black (inner) chert in } 1 \mathrm{~cm} \text { to } 1 \mathrm{ft} \\
\text { thick layers; bioclasts (crinoid ossicles, bryozoan? } \\
\text { fragments) especially notable in chert. About } 20- \\
40 \% \text { of dolostone is partly silicified, medium- to } \\
\text { medium-light-gray (outer). (Chert and partly } \\
\text { silicified dolostone not sampled for conodonts.) } \\
\text { Driller notes cave at } 699-707.5 \mathrm{ft} \text {. } \\
\text { Thin section data: } \\
680,681 \mathrm{ft} \text { : Dolostone with relict crinoid ossicles and } \\
\text { local patchy to pervasive chert replacement. } \\
709.2 \mathrm{ft} \text { : Dolostone (crystals mostly } 300-400 \mu \text { m) with } \\
\text { rare open vugs, some of which may be crinoid } \\
\text { ossicle molds. } \\
709.4 \mathrm{ft} \text { : Like } 709.2 \text {, but some vugs partly filled with } \\
\text { quartz and others contain dead oil. } \\
\text { Heavy-mineral concentrate is chiefly phosphatic } \\
\text { bioclasts (brachiopod fragments) and phosphatized } \\
\text { bioclasts and rock fragments. } \\
7.1 \text { kg of rock was processed. } \\
\text { Loc. } 9 \text { (app. } 1 \text { ) of Dumoulin and others (2004). }\end{array}$ \\
\hline
\end{tabular}


Table 6. Conodont samples from the Kogruk Formation-Continued.

[All faunas identified by A.G. Harris. Structural units determined by L.E. Young. EMA, Endicott Mountains allochthon; KRA, Kelly River allochthon. CAI, conodont color alteration index. Letters in field number refer to collector: AD, J.A. Dumoulin; AK, J.S. Kelley; AKD, K.D. Kelley; JS, J.M. Schmidt; KE, K. Evans; KS, K.W. Sherwood; MD, C.F. Mayfield; and TR, I.L. Tailleur. DDH, diamond drill hole. Interval thicknesses in drill holes are drilled thicknesses and have not been corrected for locality]

\begin{tabular}{|c|c|c|c|c|c|}
\hline $\begin{array}{l}\text { Locality No., } \\
\text { structural unit }\end{array}$ & $\begin{array}{l}\text { Quadrangle, } \\
\text { latitude } \mathrm{n} . / \\
\text { longitude w. }\end{array}$ & $\begin{array}{c}\text { Conodont fauna and CAI } \\
\text { [field No.; USGS collection No.] }\end{array}$ & Age & \begin{tabular}{|c|}
$\begin{array}{c}\text { Conodont biofacies and } \\
\text { depositional } \\
\text { environment }\end{array}$ \\
\end{tabular} & Remarks \\
\hline $\begin{array}{l}160 \\
\text { EMA (Key } \\
\text { Creek plate; } \\
\text { Port Road } \\
\text { succession of } \\
\text { Dumoulin and } \\
\text { others, 2004) } \\
\text { [cont.] }\end{array}$ & $\begin{array}{l}\text { Noatak D-3 } \\
67^{\circ} 52^{\prime} 40^{\prime \prime} / \\
163^{\circ} 11^{\prime \prime} 00^{\prime \prime}\end{array}$ & $\begin{array}{l}\text { Abundant conodonts; none are complete, a minor } \\
\text { number are identifiable to genus and (or) species, } \\
\text { and most are relatively small indeterminate } \\
\text { fragments. } \\
32 \text { incomplete to nearly complete Pa elements } \\
\text { Bispathodus utahensis Sandberg and Gutschick } \\
\text { Idioprioniodus cf. I. healdi (Roundy) } \\
7 \mathrm{~Pb} \text {, } 4 \mathrm{M}, 5 \mathrm{Sc} \text { elements } \\
\text { Kladognathus sp. indet. } \\
1 \mathrm{Pa-Pb}, 12 \mathrm{M}, 1 \mathrm{Sa} \text {, and } 35 \mathrm{Sb}-\mathrm{Sc} \text { elements (all } \\
\text { fragments) } \\
\text { Unassigned elements: } \\
4 \mathrm{~Pb} \text { and } 1 \mathrm{M} \\
655 \text { indet. bar, blade, and platform fragments } \\
\mathrm{CAI}=3 \\
\text { [DDH } 1104 \text {, composite conodont sample 2: } 713.5- \\
14,714-720 \mathrm{ft} \text { (only } 2 \mathrm{ft} \text { of section recovered in } \\
\text { this 6-ft-interval; } 1.5 \mathrm{ft} \text { section was sampled); } \\
33663-\mathrm{PC}]\end{array}$ & $\begin{array}{l}\text { latest Early-early Late } \\
\text { Mississippian (late } \\
\text { Osagean-Meramecian). } \\
\text { The overlying collection } \\
\text { restricts the upper age limit } \\
\text { of this sample to the } G n \text {. } \\
\text { homopunctatus-Upper } G n \text {. } \\
\text { texanus Zone (early } \\
\text { Meramecian). }\end{array}$ & $\begin{array}{l}\text { Post-mortem transport } \\
\text { from or within a } \\
\text { bispathodid- } \\
\text { kladognathid } \\
\text { biofacies. Breakage } \\
\text { indicates transport } \\
\text { within or from a high- } \\
\text { energy depositional } \\
\text { setting. }\end{array}$ & $\begin{array}{l}\text { Kogruk Formation, upper part, immediately below } \\
\text { DDH } 1104 \text { composite conodont sample } 1 . \\
713.5-720 \mathrm{ft} \text { : Dolostone (locally cherty) as described } \\
\text { above. } \\
\text { Heavy-mineral concentrate is chiefly partly pyritic to } \\
\text { nonpyritic phosphatic and phosphatized bioclasts } \\
\text { (including phosphatic brachiopod fragments). } \\
2.4 \text { kg of rock was processed. }\end{array}$ \\
\hline
\end{tabular}


Table 7. Conodont samples from the Utukok Formation

[All faunas identified by A.G. Harris. Structural units determined by L.E. Young. EMA, Endicott Mountains allochthon; KRA, Kelly River allochthon; PCA, Picnic Creek allochthon. CAI, conodont color alteration index. Letters in field number refer to collector: AD, J.A. Dumoulin; AK, J.S. Kelley; Cx, S.M. Curtis; EK, I. Ellersieck; JS, J.M. Schmidt; KE, K. Evans; K.S., K.W. Sherwood; and TR, I.L. Tailleur. DDH, diamond drill hole. All drill hole samples collected by J.A. Dumoulin. Lithologic data under remarks are field descriptions unless otherwise indicated; thin section observations by J.A. Dumoulin. No., number; indet., indeterminate; loc., locality]

\begin{tabular}{|c|c|c|c|c|c|}
\hline $\begin{array}{l}\text { Locality No., } \\
\text { structural unit }\end{array}$ & $\begin{array}{l}\text { Quadrangle, } \\
\text { latitude n./ } \\
\text { longitude w. }\end{array}$ & $\begin{array}{c}\text { Conodont fauna and CAI } \\
\text { [field No.; USGS collection No.] }\end{array}$ & Age & $\begin{array}{c}\text { Conodont biofacies and } \\
\text { depositional } \\
\text { environment }\end{array}$ & Remarks \\
\hline $\begin{array}{l}9 \\
\text { KRA (Kelly } \\
\text { plate) }\end{array}$ & $\begin{array}{l}\text { De Long Mtns. } \\
\text { A-3 } \\
68^{\circ} 11^{\prime} 44.6^{\prime \prime} / \\
163^{\circ} 41^{\prime} 54.4^{\prime \prime}\end{array}$ & $\begin{array}{l}3 \text { Pa elements Eotaphrus burlingtonensis Pierce } \\
\text { and Langenheim? } \\
10 \mathrm{M} \text { elements Gnathodus sp. indet. } \\
\text { Hindeodus crassidentatus (Branson and Mehl) } \\
15 \mathrm{~Pa}, 4 \mathrm{~Pb}, 1 \mathrm{M}, 2 \mathrm{Sa}, 14 \mathrm{Sb} \text {, and } 8 \mathrm{Sc} \text { elements } \\
\text { Idioprioniodus sp. indet. } \\
1 \mathrm{~Pb}, 2 \mathrm{M} \text {, and } 2 \mathrm{Sc} \text { elements } \\
\text { Kladognathus sp. } \\
24 \mathrm{M}, 12 \mathrm{Sa} \text {, and } 36 \mathrm{Sb}-\mathrm{Sc} \text { elements } \\
2 \mathrm{~Pa} \text { elements Polygnathus communis Branson and } \\
\text { Mehl } \\
\text { Synclydognathus geminus (Hinde) } \\
34 \mathrm{~Pa}, 3 \mathrm{~Pb} \text {, and } 19 \mathrm{M} \text { and S elements } \\
\text { Unassigned elements; } \\
12 \mathrm{~Pa} \text { (3 morphotypes; all fragments), } 6 \mathrm{~Pb} \text { (4 } \\
\text { morphotypes), } 10 \mathrm{M} \text { (3 morphotypes), } 7 \mathrm{Sc}(3 \\
\text { morphotypes) } \\
274 \text { indet. bar, blade, and platform fragments } \\
\text { CAI=2 } \\
\text { [00AD5A; 33626-PC] }\end{array}$ & $\begin{array}{l}\text { late Early Mississippian } \\
\quad \text { (middle-late Osagean; Sc. } \\
\text { anchoralis-D. latus Zone- } \\
\text { Po. mehli-Lower Gn. } \\
\text { texanus Zone). }\end{array}$ & $\begin{array}{l}\text { Mixed biofacies; } \\
\text { postmortem transport } \\
\text { from a range of } \\
\text { shallow-water } \\
\text { depositional } \\
\text { environments. }\end{array}$ & $\begin{array}{l}\text { Within lower part of Utukok Formation (base is a } \\
\text { fault). } \\
\text { Light-gray-weathering, medium-gray, irregularly } \\
\text { bedded skeletal supportstone with variable amounts } \\
\text { of quartz; beds } 3 \text { to } 20 \mathrm{~cm} \text { thick. Thin section is a } \\
\text { diverse skeletal grainstone with 25\% noncarbonate } \\
\text { detrital grains (mainly quartz); bioclasts include } \\
\text { brachiopods, crinoids, ostracodes, and foraminifers. } \\
\text { Heavy-mineral concentrate includes scarce } \\
\text { phosphatized bryozoan fragments, rare ichthyoliths, } \\
\text { and phosphatized foraminiferan steinkerns. } \\
9.6 \text { kg of rock was processed. }\end{array}$ \\
\hline $\begin{array}{l}18 \\
\text { Olistolith }\end{array}$ & $\begin{array}{l}\text { De Long Mtns. } \\
\text { A-3 } \\
68^{\circ} 08^{\prime} 24^{\prime \prime} / \\
163^{\circ} 25^{\prime} 48^{\prime \prime}\end{array}$ & $\begin{array}{l}1 \text { Pa element Bispathodus plumulus nodosus } \\
\text { (Rhodes, Austin, and Druce) } \\
9 \text { Pa element anterior platform fragments } \\
\text { Siphonodella sp. indet. of middle-late } \\
\text { Kinderhookian morphotype } \\
15 \text { indet. blade and platform fragments (most } \\
\text { probably small siphonodellid fragments) } \\
\text { CAI=2.5-3 } \\
\text { [79TR144; 27596-PC] }\end{array}$ & $\begin{array}{l}\text { early Early Mississippian } \\
\text { (middle-late } \\
\text { Kinderhookian). }\end{array}$ & $\begin{array}{l}\text { Siphonodellid biofacies; } \\
\text { postmortem transport } \\
\text { within or from a } \\
\text { middle shelf or } \\
\text { deeper, relatively } \\
\text { quiet water } \\
\text { depositional setting. }\end{array}$ & $\begin{array}{l}\text { This collection resembles faunules from the Rough } \\
\text { Mountain unit of the Lisburne Group (Dumoulin and } \\
\text { Harris, 1997). } \\
\text { Fossil loc. 21, Mayfield and others (1990). }\end{array}$ \\
\hline
\end{tabular}


Table 7. Conodont samples from the Utukok Formation-Continued.

[All faunas identified by A.G. Harris. Structural units determined by L.E. Young. EMA, Endicott Mountains allochthon; KRA, Kelly River allochthon; PCA, Picnic Creek allochthon. CAI, conodont color alteration index. Letters in field number refer to collector: AD, J.A. Dumoulin; AK, J.S. Kelley; Cx, S.M. Curtis; EK, I. Ellersieck; JS, J.M. Schmidt; KE, K. Evans; K.S., K.W. Sherwood; and TR, I.L. Tailleur. DDH, diamond drill hole. All drill hole samples collected by J.A. Dumoulin. Lithologic data under remarks are field descriptions unless otherwise indicated; thin section observations by J.A. Dumoulin. No., number; indet., indeterminate; loc., locality]

\begin{tabular}{|c|c|c|c|c|c|}
\hline $\begin{array}{l}\text { Locality No., } \\
\text { structural unit }\end{array}$ & $\begin{array}{l}\text { Quadrangle, } \\
\text { latitude n./ } \\
\text { longitude w. }\end{array}$ & $\begin{array}{c}\text { Conodont fauna and CAI } \\
\text { [field No.; USGS collection No.] }\end{array}$ & Age & \begin{tabular}{|c|} 
Conodont biofacies and \\
depositional \\
environment
\end{tabular} & Remarks \\
\hline $\begin{array}{l}25 \\
\text { Olistolith, } \\
\text { probably } \\
\text { derived from } \\
\text { PCA or KRA }\end{array}$ & $\begin{array}{l}\text { De Long Mtns. } \\
\text { A-3 } \\
68^{\circ} 06^{\prime} 06^{\prime \prime} / \\
163^{\circ} 34^{\prime} 18^{\prime \prime}\end{array}$ & $\begin{array}{l}3 \text { Pa elements (all incomplete) Bispathodus stabilis } \\
\text { (Branson and Mehl) or Bi. utahensis Sandberg } \\
\text { and Gutschick } \\
17 \text { Pa elements Bispathodus plumulus nodosus } \\
\text { (Rhodes, Austin, and Druce) } \\
10 \text { Pa elements Hindeodus crassidentatus (Hinde) } \\
4 \text { Sc elements Kladognathus sp. indet. } \\
7 \text { Pa elements Polygnathus bischoffi Rhodes, } \\
\text { Austin and Druce [pl. 2, figs. 20, 21] } \\
15 \text { Pa elements Polygnathus communis communis } \\
\text { Branson and Mehl and Po. c. carina Hass } \\
1 \text { Pa element (incomplete) Polygnathus vogesi } \\
1 \text { large Pa fragment Polygnathus sp. indet. } \\
2 \text { Pa elements Pseudopolygnathus marginatus } \\
\text { (Branson and Mehl)? [pl. 2, figs. 23, 24] } \\
1 \text { Pa element Pseudopolygnathus multistriatus } \\
\text { Mehl and Thomas [pl. 2, figs. 25, 26] } \\
13 \text { Pa elements (most incomplete but very large) } \\
\text { Pseudopolygnathus } \mathrm{n} \text {. sp.? of Dumoulin and } \\
\text { Harris (1997, fig. } 9 \text { H) from the Anuik River } \\
\text { sequence of the Endicott Mtns. allochthon } \\
\text { Siphonodella spp. } \\
14 \text { Pa (mostly fragments and juveniles), } 1 \text { Pb, and } \\
1 \mathrm{M} \text { elements } \\
1 \text { Sb element Synclydognathus geminus (Hinde) } \\
\text { [pl. 2, fig. 22] } \\
\text { Unassigned elements: } \\
9 \text { Pb (+5 morphotypes), 11 M (+5 morphotypes), } \\
5 \mathrm{Sa} \text { (2 morphotypes), } 2 \text { Sb (2 morphotypes), and } \\
4 \mathrm{Sc} \text { (3 morphotypes) } \\
52 \text { indet. bar, blade, and platform fragments } \\
\\
\text { CAI=3 } \\
\text { [79EK175C; 27553-PC] }\end{array}$ & $\begin{array}{l}\text { early Early Mississippian } \\
\text { (middle-late } \\
\text { Kinderhookian). }\end{array}$ & $\begin{array}{l}\text { Mixed biofacies; } \\
\text { postmortem transport } \\
\text { in a shelf depositional } \\
\text { setting. Most of the } \\
\text { conodonts (with the } \\
\text { exception of the } \\
\text { siphonodellids) } \\
\text { suggest a relatively } \\
\text { high-energy, shallow- } \\
\text { water setting. }\end{array}$ & $\begin{array}{l}\text { Bedded limestone underlying interbedded limestone } \\
\text { and chert. } \\
\text { This fauna resembles that of the Rough Mountain } \\
\text { Creek unit of the Lisburne Group (Dumoulin and } \\
\text { Harris 1997; Mull and others, 1997). } \\
\text { Fossil loc. 24, Mayfield and others (1990). }\end{array}$ \\
\hline
\end{tabular}


Table 7. Conodont samples from the Utukok Formation-Continued.

[All faunas identified by A.G. Harris. Structural units determined by L.E. Young. EMA, Endicott Mountains allochthon; KRA, Kelly River allochthon; PCA, Picnic Creek allochthon. CAI, conodont color alteration index. Letters in field number refer to collector: AD, J.A. Dumoulin; AK, J.S. Kelley; Cx, S.M. Curtis; EK, I. Ellersieck; JS, J.M. Schmidt; KE, K. Evans; K.S., K.W. Sherwood; and TR, I.L. Tailleur. DDH, diamond drill hole. All drill hole samples collected by J.A. Dumoulin. Lithologic data under remarks are field descriptions unless otherwise indicated; thin section observations by J.A. Dumoulin. No., number; indet., indeterminate; loc., locality]

\begin{tabular}{|c|c|c|c|c|c|}
\hline $\begin{array}{l}\text { Locality No., } \\
\text { structural unit }\end{array}$ & $\begin{array}{l}\text { Quadrangle, } \\
\text { latitude n./ } \\
\text { longitude w. }\end{array}$ & $\begin{array}{c}\text { Conodont fauna and CAI } \\
\text { [field No.; USGS collection No.] }\end{array}$ & Age & $\begin{array}{c}\text { Conodont biofacies and } \\
\text { depositional } \\
\text { environment }\end{array}$ & Remarks \\
\hline $\begin{array}{l}31 \\
\text { EMA (Key } \\
\text { Creek plate) }\end{array}$ & $\begin{array}{l}\text { De Long Mtns. } \\
\text { A-3 } \\
68^{\circ} 02^{\prime} 21.8^{\prime \prime} / \\
163^{\circ} 12^{\prime} 03.6^{\prime \prime}\end{array}$ & $\begin{array}{l}2 \text { incomplete Pa elements Polygnathus bischoffi } \\
\text { Rhodes, Austin, and Druce } \\
8 \text { Pa element fragments Polygnathus communis } \\
\text { communis Branson and Mehl } \\
1 \text { juvenile Pa element Polygnathus flabellus } \\
\text { Branson and Mehl? } \\
9 \text { Pa element fragments Polygnathus sp. indet. and } \\
\text { (or) Pseudopolygnathus sp. indet. } \\
1 \text { Pa element Pseudopolygnathus sp. [pl. 2, fig. 17] } \\
2 \text { juvenile Pa elements Pseudopolygnathus? sp. } \\
\text { indet. } \\
6 \text { Pa element fragments Pseudopolygnathus spp. } \\
\text { indet. } \\
\text { Synclydognathus geminus (Hinde) } \\
1 \text { Pb and } 1 \mathrm{~S} \text { elements } \\
\text { Unassigned elements: } \\
3 \text { Pb (2 morphotypes), } 1 \mathrm{M}, 3 \mathrm{Sa} \text { (2 } \\
\text { morphotypes), and } 1 \mathrm{Sc} \\
137 \text { indet. bar, blade, and platform fragments } \\
\text { CAI=3.5 } \\
\text { [00AD6A; 33627-PC] }\end{array}$ & $\begin{array}{l}\text { late Early Mississippian } \\
\text { (middle-middle late } \\
\text { Osagean). }\end{array}$ & $\begin{array}{l}\text { Postmortem transport } \\
\text { within or from a } \\
\text { pseudopolygnathid- } \\
\text { polygnathid biofacies; } \\
\text { platform or shelf } \\
\text { depositional setting. }\end{array}$ & $\begin{array}{l}\text { Lower part of Utukok Formation. } \\
\text { Orange-gray- to medium-light-gray-weathering, } \\
\text { medium-gray, partly bioturbated supportstone with } \\
\text { pelmatozoans in beds } 8 \text { to } 15 \mathrm{~cm} \text { thick. Thin section } \\
\text { is diverse skeletal supportstone with trace quartz silt; } \\
\text { bioclasts include brachiopods, crinoids, ostracodes, } \\
\text { foraminifers, gastropods, and algae(?). } \\
\text { Heavy-mineral concentrate includes micaceous, } \\
\text { quartzose metamorphic(?) rock fragments, white } \\
\text { mica flakes, and rare phosphatized bryozoan and } \\
\text { pelmatozoan fragments and ostracodes. } \\
9.9 \text { kg of rock was processed. } \\
\text { Loc. } 17 \text { (fig. 2) of Dumoulin and others (2004). }\end{array}$ \\
\hline $\begin{array}{l}40 \\
\text { KRA (Wulik } \\
\text { Peaks plate) }\end{array}$ & $\begin{array}{l}\text { De Long Mtns. } \\
\text { B-2 } \\
68^{\circ} 16^{\prime} 20.3^{\prime \prime /} \\
163^{\circ} 11^{\prime} 10.7^{\prime \prime}\end{array}$ & $\begin{array}{l}1 \text { unassigned } \mathrm{Pb} \text { element } \\
8 \text { indet. bar and blade fragments } \\
\mathrm{CAI}=2.5-3 \\
{[\mathrm{KE} 98-14 \mathrm{E} ; 33501-\mathrm{PC}]}\end{array}$ & Mississippian & $\begin{array}{l}\text { Indeterminate (too few } \\
\text { conodonts). }\end{array}$ & $\begin{array}{l}\text { Middle Utukok Formation. See table } 6 \text { for Kogruk } \\
\text { Formation samples from this location. } \\
\text { Thin section is fine-grained skeletal-peloidal } \\
\text { grainstone; bioclasts chiefly calcispheres and } \\
\text { foraminifers with a few outsized crinoid ossicles. } \\
\text { Heavy-mineral concentrate includes barite(?) grains, } \\
\text { minor phosphatized spines, and phosphatic } \\
\text { brachiopod fragments. } \\
2.9 \mathrm{~kg} \text { of rock was processed. }\end{array}$ \\
\hline
\end{tabular}


Table 7. Conodont samples from the Utukok Formation-Continued.

[All faunas identified by A.G. Harris. Structural units determined by L.E. Young. EMA, Endicott Mountains allochthon; KRA, Kelly River allochthon; PCA, Picnic Creek allochthon. CAI, conodont color alteration index. Letters in field number refer to collector: AD, J.A. Dumoulin; AK, J.S. Kelley; Cx, S.M. Curtis; EK, I. Ellersieck; JS, J.M. Schmidt; KE, K. Evans; K.S., K.W. Sherwood; and TR, I.L. Tailleur. DDH, diamond drill hole. All drill hole samples collected by J.A. Dumoulin. Lithologic data under remarks are field descriptions unless otherwise indicated; thin section observations by J.A. Dumoulin. No., number; indet., indeterminate; loc., locality]

\begin{tabular}{|c|c|c|c|c|c|}
\hline $\begin{array}{l}\text { Locality No., } \\
\text { structural unit }\end{array}$ & $\begin{array}{l}\text { Quadrangle, } \\
\text { latitude n./ } \\
\text { longitude w. }\end{array}$ & $\begin{array}{c}\text { Conodont fauna and CAI } \\
\text { [field No.; USGS collection No.] }\end{array}$ & Age & $\begin{array}{c}\text { Conodont biofacies and } \\
\text { depositional } \\
\text { environment }\end{array}$ & Remarks \\
\hline 51 & $\begin{array}{l}\text { De Long Mtns. } \\
\text { A-2 } \\
68^{\circ} 12^{\prime} 42^{\prime \prime} / \\
163^{\circ} 10^{\prime} 42^{\prime \prime}\end{array}$ & $\begin{array}{l}9 \text { indet. small bar and blade fragments } \\
\mathrm{CAI}=3 \\
{[\mathrm{KS} 98-85 ; 33606-\mathrm{PC}]}\end{array}$ & $\begin{array}{l}\text { Ordovician-Triassic on the } \\
\text { basis of conodonts; } \\
\text { Mississippian on the basis } \\
\text { of stratigraphic unit. }\end{array}$ & $\begin{array}{l}\text { Indeterminate (too few } \\
\text { conodonts). }\end{array}$ & $\begin{array}{l}\text { Lower Utukok Formation. } \\
\text { Carbonate tempestites, } 10 \text { to } 40 \mathrm{~cm} \text { thick ( } 40 \%) \text {, } \\
\text { interbedded with gray calcareous shale. Thin section } \\
\text { is calcite>quartz siltstone with locally abundant } \\
\text { calcareous spicules, lesser calcispheres, algae, and } \\
\text { outsized crinoid and bryozoan fragments. Non- } \\
\text { carbonate detritus 5-30\%; chiefly quartz but includes } \\
\text { plagioclase feldspar. } \\
\text { Heavy-mineral concentrate contains scarce phosphatic } \\
\text { shell fragments. } \\
5.1 \text { kg of rock was processed. }\end{array}$ \\
\hline $\begin{array}{l}52 \\
\text { KRA (Wulik } \\
\text { Peaks plate) }\end{array}$ & $\begin{array}{l}\text { De Long Mtns. } \\
\text { A-2 } \\
68^{\circ} 12^{\prime} 24^{\prime \prime} / \\
163^{\circ} 11^{\prime} 48^{\prime \prime}\end{array}$ & $\begin{array}{l}\text { Bispathodus utahensis Sandberg and Gutschick } \\
52 \mathrm{~Pa} \text { and } 1 \mathrm{M} \text { elements [pl. 3, fig. 2] } \\
\text { Kladognathus tenuis (Branson and Mehl) } \\
3 \mathrm{~Pa}-\mathrm{Pb} \text { element fragments, } 5 \mathrm{M}, 2 \mathrm{Sa} \text {, and } 15 \mathrm{Sb}- \\
\text { Sc elements } \\
1 \text { juvenile Pa element Pseudopolygnathus sp. [pl. 3, } \\
\text { fig. 1] } \\
1 \text { Sa element Synclydognathus sp. } \\
5 \text { Pa elements Vogelgnathus cf. V. pesaquidi } \\
\text { Purnell and von Bitter } \\
\text { Unassigned digyrate apparatus: } \\
1 \mathrm{P} \text { and } 2 \text { Sb-Sc elements } \\
\text { Unassigned elements: } \\
6 \mathrm{M} \text { (3 morphotypes), } 4 \mathrm{Sa} \text { (3 morphotypes), } 3 \mathrm{Sb} \\
\text { (3 morphotypes), and } 13 \mathrm{Sc} \text { (5 morphotypes) } \\
232 \text { indet. bar, blade, and platform fragments } \\
\text { CAI=2.5-3 } \\
\text { [97AK107A; 33400-PC] }\end{array}$ & $\begin{array}{l}\text { late Early Mississippian } \\
\text { (middle-late Osagean); the } \\
\text { occurrence of a } \\
\text { pseudopolygnathid with } \\
\text { Vogelgnathus cf. } V \text {. } \\
\text { pesaquidi restricts the age } \\
\text { of the collection to the } \\
\text { middle or late Osagean. } \\
\text { According to Purnell and } \\
\text { von Bitter (1992), } V \text {. } \\
\text { pesaquidi and any } \\
\text { vogelgnathids like it do not } \\
\text { appear below the middle } \\
\text { Osagean, and } \\
\text { Pseudopolygnathus does } \\
\text { not range above the } \\
\text { Osagean. }\end{array}$ & $\begin{array}{l}\text { Bispathodid biofacies; } \\
\text { this is a eurytopic } \\
\text { species that is most } \\
\text { common in shallow- } \\
\text { and deep-water } \\
\text { environments. The } \\
\text { associated conodonts } \\
\text { suggest a shallow- } \\
\text { water depositional } \\
\text { setting. }\end{array}$ & $\begin{array}{l}\text { Top of Utukok Formation. } \\
\text { Medium-light to medium-gray-weathering, medium- } \\
\text { dark-gray to medium-brown-gray, coarse to very } \\
\text { coarse grained limestone. Beds } 10 \text { - to } 40 \text {-cm-thick } \\
\text { with dark-gray argillaceous partings, some cross- } \\
\text { laminae, and local chert nodules. Thin section is very } \\
\text { poorly sorted skeletal packstone; bioclasts (to } 7 \mathrm{~mm} \text { ) } \\
\text { include crinoid ossicles (50\%), brachiopods, } \\
\text { bryozoans, ostracodes, and a few foraminifers. Some } \\
\text { shelter porosity; fair amount of diagenetic alteration, } \\
\text { including silica replacement of bioclasts (and } \\
\text { locally, matrix) and dolomite replacement of mud } \\
\text { matrix (mostly rhombohedral dolomite rhombs }<60 \\
\mu \text { m in diameter). } \\
\text { Heavy-mineral concentrate is chiefly phosphatic and } \\
\text { phosphatized bioclasts including ichthyoliths, } \\
\text { bryozoans, and brachiopods. } \\
14.1 \text { kg of rock was processed. }\end{array}$ \\
\hline
\end{tabular}


Table 7. Conodont samples from the Utukok Formation-Continued.

[All faunas identified by A.G. Harris. Structural units determined by L.E. Young. EMA, Endicott Mountains allochthon; KRA, Kelly River allochthon; PCA, Picnic Creek allochthon. CAI, conodont color alteration index. Letters in field number refer to collector: AD, J.A. Dumoulin; AK, J.S. Kelley; Cx, S.M. Curtis; EK, I. Ellersieck; JS, J.M. Schmidt; KE, K. Evans; K.S., K.W. Sherwood; and TR, I.L. Tailleur. DDH, diamond drill hole. All drill hole samples collected by J.A. Dumoulin. Lithologic data under remarks are field descriptions unless otherwise indicated; thin section observations by J.A. Dumoulin. No., number; indet., indeterminate; loc., locality]

\begin{tabular}{|c|c|c|c|c|c|}
\hline $\begin{array}{l}\text { Locality No., } \\
\text { structural unit }\end{array}$ & $\begin{array}{l}\text { Quadrangle, } \\
\text { latitude n./ } \\
\text { longitude w. }\end{array}$ & $\begin{array}{c}\text { Conodont fauna and CAI } \\
\text { [field No.; USGS collection No.] }\end{array}$ & Age & \begin{tabular}{|c|}
$\begin{array}{c}\text { Conodont biofacies and } \\
\text { depositional } \\
\text { environment }\end{array}$ \\
\end{tabular} & Remarks \\
\hline \multirow[t]{2}{*}{$\begin{array}{l}53 \\
\text { KRA (Wulik } \\
\text { Peaks plate) }\end{array}$} & \multirow[t]{2}{*}{$\begin{array}{l}\text { De Long Mtns. } \\
\text { A-2 } \\
68^{\circ} 12^{\prime} 17^{\prime \prime} / \\
163^{\circ} 08^{\prime} 39^{\prime \prime}\end{array}$} & $\begin{array}{l}\text { Bispathodus stabilis (Branson and Mehl) or Bi. } \\
\text { utahensis Sandberg and Gutschick } \\
3 \text { incomplete } \mathrm{Pa} \text { and } 1 \mathrm{~Pb} \text { elements } \\
\text { Synclydognathus sp. indet. } \\
6 \mathrm{~Pa} \text { (fragments) and } 1 \mathrm{~S} \text { or M (small fragment) } \\
\text { elements } \\
\text { digyrate apparatus } \\
1 \mathrm{~Pa}-\mathrm{Pb} \text { and } 3 \mathrm{Sb}-\mathrm{Sc} \text { element fragments } \\
2 \text { unassigned } \mathrm{M} \text { elements ( } 2 \text { morphotypes) } \\
1 \text { unassigned } \mathrm{Sa} \text { or Sb element fragment } \\
38 \text { indet. bar, blade, and platform fragments } \\
\text { CAI=3 } \\
\text { [97AK96A; 33397-PC] }\end{array}$ & \begin{tabular}{|l} 
Early, but not earliest \\
Mississippian (middle \\
Kinderhookian-Osagean).
\end{tabular} & $\begin{array}{l}\text { Indeterminate (too few } \\
\text { generically } \\
\text { determinate } \\
\text { conodonts). }\end{array}$ & $\begin{array}{l}\text { Top of 250-ft-thick interval within Utukok Formation } \\
\text { that resembles Kogruk Formation in lithofacies. } \\
\text { Medium-light-gray-weathering, medium- to medium- } \\
\text { dark-gray limestone in 5- to 50-cm-thick beds. Thin } \\
\text { section is loosely packed, coarse skeletal grainstone } \\
\text { bioclasts dominantly crinoid ossicles and algae, also } \\
\text { brachiopods, foraminifers, ostracodes, and } \\
\text { echinoderm spines. Skeletal pores filled with calcite } \\
\text { spar. Trace dolomite ( } 20 \mu \mathrm{m} \text { rhombs) replacing } \\
\text { minor amounts of calcite mud(?). } \\
7.1 \mathrm{~kg} \text { of rock was processed. }\end{array}$ \\
\hline & & $\begin{array}{l}\text { Hindeodus crassidentatus (Branson and Mehl) } \\
8 \mathrm{~Pa}, 3 \mathrm{~Pb}, 9 \mathrm{M}, 1 \mathrm{Sa} \text {, and } 2 \mathrm{Sc} \text { elements } \\
\text { Kladognathus sp. } \\
3 \mathrm{Sa} \text { and } 11 \mathrm{Sb}-\mathrm{Sc} \text { elements } \\
\text { Unassigned elements: } \\
4 \mathrm{~Pb}(3 \text { morphotypes) and } 2 \mathrm{Sc} \\
28 \text { indet. bar, blade, and platform fragments } \\
\mathrm{CAI}=3 \\
\text { [97AK96B; 33398-PC] }\end{array}$ & \begin{tabular}{|l} 
Early, but not earliest \\
Mississippian (middle \\
Kinderhookian-Osagean, \\
possibly late Osagean).
\end{tabular} & $\begin{array}{l}\text { Hindeodid biofacies; } \\
\text { relatively shallow } \\
\text { water, normal-marine } \\
\text { to near restricted } \\
\text { depositional setting. }\end{array}$ & $\begin{array}{l}\text { Base of } 250 \text {-ft-thick interval within Utukok Formation } \\
\text { that resembles Kogruk Formation in lithofacies. } \\
\text { Limestone similar to 97AK96A. Thin section is } \\
\text { skeletal grainstone like 97AK96A but more closely } \\
\text { packed, better sorted, finer grained (bioclasts mostly } \\
\text { fine grained, some to } 2 \text { mm). Grains include crinoid } \\
\text { ossicles, foraminifers, algae, brachiopods, and } \\
\text { ostracodes; } 10 \% \text { of bioclasts micritized. Some } \\
\text { skeletal pores filled with micrite, some with calcite } \\
\text { spar. Minor (<1\%) detrital monocrystalline quartz } \\
\text { silt to very fine sand. } \\
\text { Heavy-mineral concentrate is chiefly phosphatized } \\
\text { bioclasts (mainly pelmatozoan fragments and } \\
\text { slightly curved irregular tubes). } \\
7.2 \text { kg of rock was processed. }\end{array}$ \\
\hline
\end{tabular}


Table 7. Conodont samples from the Utukok Formation-Continued.

[All faunas identified by A.G. Harris. Structural units determined by L.E. Young. EMA, Endicott Mountains allochthon; KRA, Kelly River allochthon; PCA, Picnic Creek allochthon. CAI, conodont color alteration index. Letters in field number refer to collector: AD, J.A. Dumoulin; AK, J.S. Kelley; Cx, S.M. Curtis; EK, I. Ellersieck; JS, J.M. Schmidt; KE, K. Evans; K.S., K.W. Sherwood; and TR, I.L. Tailleur. DDH, diamond drill hole. All drill hole samples collected by J.A. Dumoulin. Lithologic data under remarks are field descriptions unless otherwise indicated; thin section observations by J.A. Dumoulin. No., number; indet., indeterminate; loc., locality]

\begin{tabular}{|c|c|c|c|c|c|}
\hline $\begin{array}{l}\text { Locality No., } \\
\text { structural unit }\end{array}$ & $\begin{array}{l}\text { Quadrangle, } \\
\text { latitude n./ } \\
\text { longitude w. }\end{array}$ & $\begin{array}{c}\text { Conodont fauna and CAI } \\
\text { [field No.; USGS collection No.] }\end{array}$ & Age & $\begin{array}{c}\text { Conodont biofacies and } \\
\text { depositional } \\
\text { environment }\end{array}$ & Remarks \\
\hline $\begin{array}{l}53 \\
\text { KRA (Wulik } \\
\text { Peaks plate) } \\
\text { [cont.] }\end{array}$ & $\begin{array}{l}\text { De Long Mtns. } \\
\text { A-2 } \\
68^{\circ} 12^{\prime} 17^{\prime \prime} / \\
163^{\circ} 08^{\prime} 39^{\prime \prime}\end{array}$ & $\begin{array}{l}5 \text { Pa element fragments Bispathodus stabilis } \\
\text { (Branson and Mehl) or Bi. utahensis Sandberg } \\
\text { and Gutschick } \\
1 \text { Pa element fragment Polygnathus sp. indet. } \\
1 \text { Sa element Hindeodus sp. indet. } \\
\text { Unassigned elements: } \\
2 \mathrm{M}, 3 \mathrm{Sa}, 1 \mathrm{Sb} \text {, and } 3 \mathrm{Sc} \text { elements } \\
20 \text { indet. bar, blade, and platform fragments } \\
\text { CAI= 3 } \\
\text { [97AK96C; 33399-PC] }\end{array}$ & \begin{tabular}{|l|} 
Early but not earliest \\
Mississippian (middle \\
Kinderhookian-Osagean)
\end{tabular} & $\begin{array}{l}\text { Indeterminate (too few } \\
\text { conodonts); conodonts } \\
\text { present indicate a } \\
\text { normal-marine, shelf } \\
\text { or platform } \\
\text { depositional setting. }\end{array}$ & $\begin{array}{l}\text { Utukok Formation, } \sim 80 \mathrm{ft} \text { above } 97 \mathrm{AK} 96 \mathrm{~A} . \\
\text { Yellow-gray to pale yellow-brown-weathering, dark- } \\
\text { gray, impure limestone with abundant corals and } \\
\text { brachiopods and local Zoophycus traces. Beds } 1 \text { to } \\
10 \mathrm{~cm} \text { thick. Thin section is skeletal grainstone } \\
\text { similar to } 97 \mathrm{AK} 96 \mathrm{~A}, \text { with a poorly sorted mix of } \\
\text { bigger crinoid ossicles and smaller algae; traces of } \\
\text { quartz silt and minor dolomite rhombs. } \\
\text { Heavy-mineral concentrate includes fluorite, } \\
\text { phosphatic brachiopod fragments, and ichthyoliths. } \\
17.0 \text { kg of rock was processed. }\end{array}$ \\
\hline $\begin{array}{l}54 \\
\text { KRA (Wulik } \\
\text { Peaks plate) }\end{array}$ & \begin{tabular}{|l} 
De Long Mtns. \\
A-2 \\
$68^{\circ} 12^{\prime} 18.2^{\prime \prime} /$ \\
$163^{\circ} 08^{\prime} 10^{\prime \prime}$
\end{tabular} & $\begin{array}{l}\text { Barren } \\
{[97 \mathrm{AK} 99 \mathrm{~A}]}\end{array}$ & & & $\begin{array}{l}\text { Lower part of Utukok Formation ( 160 ft above base). } \\
\text { Thin section is muddy quartz siltstone with } \\
\text { noncalcareous mud and calcite cement. Clasts } \\
\text { mostly monocrystalline quartz, lesser crinoid } \\
\text { ossicles (to } 400 \mu \mathrm{m}) \text {, white mica, and rare chert(?). } \\
7.0 \mathrm{~kg} \text { of rock was processed. }\end{array}$ \\
\hline $\begin{array}{l}55 \\
\text { KRA (Wulik } \\
\text { Peaks plate) }\end{array}$ & $\begin{array}{l}\text { De Long Mtns. } \\
\text { A-2 } \\
68^{\circ} 11^{\prime} 49^{\prime \prime} / \\
163^{\circ} 10^{\prime} 19^{\prime \prime}\end{array}$ & $\begin{array}{l}4 \text { indet. bar and blade fragments } \\
\text { CAI }=4 \\
{[98 J S 10 A ; 33613-P C]}\end{array}$ & $\begin{array}{l}\text { Ordovician-Permian; unit is } \\
\text { known to be Mississippian. }\end{array}$ & $\begin{array}{l}\text { Indeterminate (too few } \\
\text { conodonts). }\end{array}$ & $\begin{array}{l}\text { Near base of Utukok Formation. } \\
\text { Orange-weathering muddy limestone with } \\
\text { brachiopods, hummocky cross-beds, and 30\% } \\
\text { calcareous shale interbeds. Thin section is coarse- } \\
\text { grained crinoidal packstone with minor quartz silt. } \\
\text { Heavy-mineral concentrate includes phosphatized } \\
\text { composite grains, lesser phosphatic brachiopod } \\
\text { fragments, and rare ichthyoliths. } \\
5.7 \mathrm{~kg} \text { of rock was processed. }\end{array}$ \\
\hline
\end{tabular}


Table 7. Conodont samples from the Utukok Formation-Continued.

[All faunas identified by A.G. Harris. Structural units determined by L.E. Young. EMA, Endicott Mountains allochthon; KRA, Kelly River allochthon; PCA, Picnic Creek allochthon. CAI, conodont color alteration index. Letters in field number refer to collector: AD, J.A. Dumoulin; AK, J.S. Kelley; Cx, S.M. Curtis; EK, I. Ellersieck; JS, J.M. Schmidt; KE, K. Evans; K.S., K.W. Sherwood; and TR, I.L. Tailleur. DDH, diamond drill hole. All drill hole samples collected by J.A. Dumoulin. Lithologic data under remarks are field descriptions unless otherwise indicated; thin section observations by J.A. Dumoulin. No., number; indet., indeterminate; loc., locality]

\begin{tabular}{|c|c|c|c|c|c|}
\hline $\begin{array}{l}\text { Locality No., } \\
\text { structural unit }\end{array}$ & $\begin{array}{l}\text { Quadrangle, } \\
\text { latitude } \mathrm{n} . / \\
\text { longitude w. }\end{array}$ & $\begin{array}{c}\text { Conodont fauna and CAI } \\
\text { [field No.; USGS collection No.] }\end{array}$ & Age & $\begin{array}{c}\text { Conodont biofacies and } \\
\text { depositional } \\
\text { environment }\end{array}$ & Remarks \\
\hline $\begin{array}{l}56 \\
\text { KRA (Wulik } \\
\text { Peaks plate) }\end{array}$ & $\begin{array}{l}\text { De Long Mtns. } \\
\text { A-2 } \\
68^{\circ} 11^{\prime} 34.5^{\prime \prime /} / \\
163^{\circ} 10^{\prime} 0.8^{\prime \prime}\end{array}$ & \begin{tabular}{|l} 
Barren \\
[KE98-10C]
\end{tabular} & & & $\begin{array}{l}\text { Lower part of middle unit of Utukok Formation. See } \\
\text { table } 6 \text { for Kogruk Formation sample from this } \\
\text { locality. } \\
\text { Medium-light-gray- to light-brown-gray-weathering, } \\
\text { medium-dark-gray, very fine grained limestone. } \\
\text { Thin section is fine-grained skeletal grainstone with } \\
\sim 5 \% \text { detrital quartz and notable calcareous sponge } \\
\text { spicules and calcispheres. } \\
\text { Heavy-mineral concentrate includes spines and rare } \\
\text { silicified ostracodes with pyrite. } \\
4.4 \text { kg of rock was processed. } \\
\end{array}$ \\
\hline 57 & $\begin{array}{l}\text { De Long Mtns. } \\
\text { A-2 } \\
68^{\circ} 11^{\prime} 24^{\prime \prime} / \\
163^{\circ} 09^{\prime} 03.6^{\prime \prime}\end{array}$ & $\begin{array}{l}\text { Kladognathus sp. } \\
2 \mathrm{M} \text { and } 3 \mathrm{Sb} \text {-Sc elements } \\
1 \mathrm{Sb} \text { element Synclydognathus geminus (Hinde) } \\
\text { Unassigned elements: } \\
1 \mathrm{Sb} \text { and } 1 \mathrm{Sc} \\
30 \text { indet. bar, blade, and platform fragments } \\
\text { CAI=3 } \\
\text { [98JS-8A; 33610-PC] }\end{array}$ & $\begin{array}{l}\text { late Early to middle late Late } \\
\text { Mississippian (Osagean- } \\
\text { early Chesterian); } \\
\text { associated coral indicates } \\
\text { Late Mississippian (see } \\
\text { Remarks). }\end{array}$ & $\begin{array}{l}\text { Indeterminate (too few } \\
\text { conodonts). The } \\
\text { preservation of the } \\
\text { conodonts suggests } \\
\text { significant } \\
\text { postmortem transport. }\end{array}$ & $\begin{array}{l}\text { Transitional contact between Utukok Formation and } \\
\text { overlying Kogruk Formation. } \\
\text { Light-orange-brown-weathering, locally fossiliferous } \\
\text { limestone with corals, brachiopods, and crinoids, } \\
\text { interbedded with light-gray lime grainstone with rare } \\
\text { chert. Thin section is skeletal packstone with } \\
\text { algae(?), pelmatozoan fragments (some partly } \\
\text { silicified), and possible dissolved ooids replaced by } \\
\text { calcite spar. } \\
\text { Heavy-mineral concentrate includes ichthyoliths, lesser } \\
\text { phosphatic brachiopod fragments, phosphatized } \\
\text { bioclasts (mainly crinoid ossicles and tube } \\
\text { steinkerns), and fluorite. } \\
6.0 \text { kg of rock was processed. } \\
\text { Lithostrotion (Siphonodendron) sp. of Late } \\
\text { Mississippian (Meramecian-Chesterian) age occurs } \\
\text { in these strata (R.B. Blodgett, Oregon State } \\
\text { University, unpublished fossil report, 1999). }\end{array}$ \\
\hline $\begin{array}{l}62 \\
\text { KRA (Wulik } \\
\text { Peaks plate) }\end{array}$ & $\begin{array}{l}\text { De Long Mtns. } \\
\text { A-2 } \\
68^{\circ} 10^{\prime} 51 " / \\
163^{\circ} 10^{\prime} 25.6^{\prime \prime}\end{array}$ & $\begin{array}{l}\text { Hindeodus cristulus (Youngquist and Miller) } \\
2 \mathrm{~Pa}, 1 \mathrm{~Pb}, 1 \mathrm{M}, 1 \mathrm{Sa}-\mathrm{Sb} \text {, and } 1 \mathrm{Sc} \text { elements } \\
5 \mathrm{Sb}-\mathrm{Sc} \text { elements Kladognathus } \mathrm{sp} . \\
\text { Unassigned elements: } \\
1 \mathrm{~Pb} \text { and } 1 \mathrm{Sc} \\
28 \text { indet. bar, blade, and platform fragments } \\
\text { CAI=3 } \\
\text { [98JS7H; 33608-PC] }\end{array}$ & $\begin{array}{l}\text { latest Early-early Late } \\
\text { Mississippian (late } \\
\text { Osagean-Meramecian). }\end{array}$ & \begin{tabular}{|l|} 
Indeterminate (too few \\
generically \\
identifiable \\
conodonts); conodonts \\
present indicate post- \\
mortem transport \\
within or from a \\
relatively high- \\
energy, near restricted \\
depositional setting.
\end{tabular} & $\begin{array}{l}\text { See tables } 6 \text { and } 9 \text { for additional samples from this } \\
\text { locality. } \\
\text { Sample from mound-shaped bodies, } \sim 1-1.5 \mathrm{~m} \text { high, } \\
\text { made of crinoid grainstone and local algae(?). Thin } \\
\text { section is fine-grained, skeletal peloidal grainstone } \\
\text { with foraminifers and micritized bioclasts including } \\
\text { outsized bryozoan and pelmatozoan fragments. } \\
\text { Heavy-mineral concentrate is chiefly fluorite and } \\
\text { phosphatized and dolomitized carbonate grains and } \\
\text { bioclasts (mainly pelmatozoan ossicles). } \\
7.32 \text { kg of rock was processed. }\end{array}$ \\
\hline
\end{tabular}


Table 7. Conodont samples from the Utukok Formation-Continued.

[All faunas identified by A.G. Harris. Structural units determined by L.E. Young. EMA, Endicott Mountains allochthon; KRA, Kelly River allochthon; PCA, Picnic Creek allochthon. CAI, conodont color alteration index. Letters in field number refer to collector: AD, J.A. Dumoulin; AK, J.S. Kelley; Cx, S.M. Curtis; EK, I. Ellersieck; JS, J.M. Schmidt; KE, K. Evans; K.S., K.W. Sherwood; and TR, I.L. Tailleur. DDH, diamond drill hole. All drill hole samples collected by J.A. Dumoulin. Lithologic data under remarks are field descriptions unless otherwise indicated; thin section observations by J.A. Dumoulin. No., number; indet., indeterminate; loc., locality]

\begin{tabular}{|c|c|c|c|c|c|}
\hline $\begin{array}{l}\text { Locality No., } \\
\text { structural unit }\end{array}$ & $\begin{array}{l}\text { Quadrangle, } \\
\text { latitude n./ } \\
\text { longitude w. }\end{array}$ & $\begin{array}{c}\text { Conodont fauna and CAI } \\
\text { [field No.; USGS collection No.] }\end{array}$ & Age & \begin{tabular}{|c|}
$\begin{array}{c}\text { Conodont biofacies and } \\
\text { depositional } \\
\text { environment }\end{array}$ \\
\end{tabular} & Remarks \\
\hline $\begin{array}{l}63 \\
\text { EMA } \\
\text { (Wolverine } \\
\text { Creek plate; } \\
\text { Rok window) }\end{array}$ & $\begin{array}{l}\text { De Long Mtns. } \\
\text { A-2 } \\
68^{\circ} 08^{\prime} 45.6^{\prime \prime} / \\
163^{\circ} 05^{\prime} 42.6^{\prime \prime}\end{array}$ & $\begin{array}{l}\text { Hindeodus crassidentatus (Branson and Mehl) } \\
6 \mathrm{~Pa}, 3 \mathrm{~Pb}, 2 \mathrm{M}, 1 \mathrm{Sa}, 2 \mathrm{Sb} \text { and } 1 \mathrm{Sc} \text { elements [pl. } \\
2 \text {, fig. } 27] \\
\text { Kladognathus } \mathrm{sp} . \\
5 \mathrm{M} \text { and } 19 \mathrm{Sb}-\mathrm{Sc} \text { (all incomplete) elements } \\
\text { Synclydognathus geminus (Hinde) } \\
4 \mathrm{~Pa}, 1 \mathrm{M}, 1 \mathrm{Sa}, 1 \mathrm{Sb} 1,5 \mathrm{Sc} \text {, and } 3 \text { indet. S } \\
\text { elements } \\
1 \mathrm{Sb} \text { and } 1 \mathrm{Sc} \text { unassigned elements } \\
142 \text { small indet. bar, blade, and platform fragments } \\
\\
\text { CAI=3.5 } \\
{[\text { KE98-22; 33506-PC] }}\end{array}$ & $\begin{array}{l}\text { late Early Mississippian } \\
\text { (Osagean). }\end{array}$ & $\begin{array}{l}\text { Postmortem transport } \\
\text { within the hindeodid- } \\
\text { synclydognathid } \\
\text { biofacies; species } \\
\text { association suggests } \\
\text { relatively shallow- } \\
\text { water, near and (or) } \\
\text { intermittently } \\
\text { restricted depositional } \\
\text { setting. }\end{array}$ & $\begin{array}{l}\text { Sample from lens-shaped outcrops of limestone and } \\
\text { shale. Thin section is packed crinoid-bryozoan } \\
\text { grainstone with thin layers of packstone; other } \\
\text { bioclasts include ostracode valves, calcareous } \\
\text { spicules, and possible brachiopod fragments. } \\
\text { Heavy-mineral concentrate is chiefly coarsely } \\
\text { phosphatized and dolomitized mostly indeterminate } \\
\text { bioclasts (spines and (or) spicules), minor scarce } \\
\text { phosphatic brachiopod fragments, and ichthyoliths. } \\
6.4 \text { kg of rock was processed. } \\
\text { Loc. } 13 \text { (fig. 2) of Dumoulin and others (2004). }\end{array}$ \\
\hline \multirow[t]{2}{*}{$\begin{array}{l}64 \\
\text { EMA } \\
\text { (Wolverine } \\
\text { Creek plate; } \\
\text { Rok window) }\end{array}$} & \multirow[t]{2}{*}{$\begin{array}{l}\text { DeLong Mtns. } \\
\text { A-2 } \\
68^{\circ} 08^{\prime} 51^{\prime \prime} / \\
163^{\circ} 05^{\prime} 13.8^{\prime \prime}\end{array}$} & $\begin{array}{l}\text { Hindeodus crassidentatus (Branson and Mehl) } \\
3 \mathrm{~Pa} \text { and } 1 \mathrm{M} \text { elements [pl. 2, fig. 28] } \\
\text { Kladognathus sp. indet. } \\
1 \mathrm{Sa} \text { and } 2 \mathrm{Sb}-\mathrm{Sc} \text { elements } \\
\text { Synclydognathus geminus (Hinde) } \\
2 \text { Pa and } 4 \mathrm{~S} \text { and } \mathrm{M} \text { elements [pl. 2, fig. 29] } \\
1 \text { unassigned M element } \\
11 \text { indet. bar, blade, and platform fragments } \\
\text { CAI=3-4 (much organic matter, so CAI cannot be } \\
\text { precisely fixed). } \\
\text { [79CX119A; 33348-PC] }\end{array}$ & $\begin{array}{l}\text { late Early Mississippian } \\
\text { (Osagean). }\end{array}$ & $\begin{array}{l}\text { Postmortem transport } \\
\text { within or from a } \\
\text { shallow-water, } \\
\text { relatively high-energy } \\
\text { depositional setting; } \\
\text { mixed biofacies. }\end{array}$ & $\begin{array}{l}\text { Limestone; stratigraphic position within the Utukok } \\
\text { Formation unknown. } \\
2.7 \mathrm{~kg} \text { of rock was processed. } \\
\text { Fossil loc. 11, Ellersieck and others (1990). }\end{array}$ \\
\hline & & $\begin{array}{l}\text { Hindeodus crassidentatus (Branson and Mehl) } \\
1 \mathrm{~Pa}, 4 \mathrm{M} \text {, and } 1 \mathrm{Sc} \text { elements } \\
\text { Kladognathus sp. indet. } \\
2 \mathrm{Sa} \text { and } 2 \mathrm{Sb}-\mathrm{Sc} \text { elements } \\
\text { Synclydognathus geminus (Hinde) } \\
12 \mathrm{~Pa}, 4 \mathrm{~Pb} \text {, and } 4 \mathrm{~S} \text { and } \mathrm{M} \text { elements } \\
2 \text { unassigned Sc elements } \\
55 \text { indet. bar, blade, and platform fragments } \\
6 \text { ichthyoliths } \\
\text { CAI=4 } \\
\text { [8-16-83F; 29232-PC] }\end{array}$ & $\begin{array}{l}\text { late Early Mississippian } \\
\text { (Osagean). }\end{array}$ & \begin{tabular}{|l} 
Synclydognathid- \\
hindeodid biofacies; \\
relatively shallow- \\
water and possibly \\
near-restricted \\
depositional setting.
\end{tabular} & $\begin{array}{l}\text { Sample near base of Utukok Formation. } \\
\text { Medium-dark-gray, fine- to medium-grained, crinoid- } \\
\text { bearing packstone containing chert and black shale } \\
\text { (60-70\% limestone and } 40-30 \% \text { shale and chert). } \\
\text { Thin section is packed skeletal packstone made up of } \\
\text { ferroan and nonferroan calcite with irregular masses } \\
\text { of ferroan dolomite (crystals }<100 \mu \mathrm{m}) \text {. Bioclasts } \\
\text { mostly }(60-80 \%) \text { pelmatozoan fragments (to } 3 \mathrm{~mm} \text { ), } \\
\text { also bryozoan, coral, red algae(?), and trilobite(?) } \\
\text { fragments. Matrix of brown mud and chert with } \\
\text { abundant tiny calcareous spicules. } \\
5.4 \text { kg of rock was processed. } \\
\text { Sample collected by A.G. Harris. }\end{array}$ \\
\hline
\end{tabular}


Table 7. Conodont samples from the Utukok Formation-Continued.

[All faunas identified by A.G. Harris. Structural units determined by L.E. Young. EMA, Endicott Mountains allochthon; KRA, Kelly River allochthon; PCA, Picnic Creek allochthon. CAI, conodont color alteration index. Letters in field number refer to collector: AD, J.A. Dumoulin; AK, J.S. Kelley; Cx, S.M. Curtis; EK, I. Ellersieck; JS, J.M. Schmidt; KE, K. Evans; K.S., K.W. Sherwood; and TR, I.L. Tailleur. DDH, diamond drill hole. All drill hole samples collected by J.A. Dumoulin. Lithologic data under remarks are field descriptions unless otherwise indicated; thin section observations by J.A. Dumoulin. No., number; indet., indeterminate; loc., locality]

\begin{tabular}{|c|c|c|c|c|c|}
\hline $\begin{array}{l}\text { Locality No., } \\
\text { structural unit }\end{array}$ & $\begin{array}{l}\text { Quadrangle, } \\
\text { latitude n./ } \\
\text { longitude w. }\end{array}$ & $\begin{array}{c}\text { Conodont fauna and CAI } \\
\text { [field No.; USGS collection No.] }\end{array}$ & Age & $\begin{array}{c}\text { Conodont biofacies and } \\
\text { depositional } \\
\text { environment }\end{array}$ & Remarks \\
\hline $\begin{array}{l}70 \\
\text { KRA (Kelly } \\
\text { plate) }\end{array}$ & $\begin{array}{l}\text { De Long Mtns. } \\
\text { A-2 } \\
68^{\circ} 09^{\prime} 13.6^{\prime \prime} / \\
162^{\circ} 37^{\prime} 31^{\prime \prime}\end{array}$ & $\begin{array}{l}1 \text { incomplete Pa element Bispathodus stabilis } \\
\text { (Branson and Mehl) or Bi. utahensis Sandberg } \\
\text { and Gutschick } \\
\text { Kladognathus sp. } \\
1 \mathrm{M}, 2 \mathrm{Sa} \text {, and } 5 \mathrm{Sb}-\mathrm{Sc} \text { elements (all incomplete) } \\
1 \text { incomplete Pa element Polygnathus communis } \\
\text { Branson and Mehl } \\
\text { Synclydognathus libratus (Varker) } \\
1 \text { Pa, } 1 \mathrm{~Pb} \text {, and } 12 \mathrm{~S} \text { and M elements (all } \\
\text { incomplete) } \\
1 \mathrm{Sa} \text { and } 1 \mathrm{Sc} \text { unassigned elements } \\
80 \text { indet. bar, blade, and platform fragments } \\
\text { (mostly small) } \\
\text { CAI=3.5 } \\
\text { [00AD1B; 33623-PC] }\end{array}$ & $\begin{array}{l}\text { late Early Mississippian } \\
\text { (Osagean). }\end{array}$ & $\begin{array}{l}\text { Postmortem transport } \\
\text { within or from a } \\
\text { relatively high- } \\
\text { energy, shallow-water } \\
\text { environment. }\end{array}$ & $\begin{array}{l}\text { Within lower part of Utukok Formation; base of } \\
\text { Utukok is a fault. } \\
\text { Light-gray- to medium-light-gray-weathering, light- to } \\
\text { medium-light-gray, thick- to medium-bedded partly } \\
\text { muddy and bioturbated supportstone with abundant } \\
\text { to common pelmatozoan ossicles. Thin section is } \\
\text { skeletal grainstone with pelmatozoan debris, } \\
\text { foraminifers, and algae, plus a few percent detrital } \\
\text { quartz and plagioclase feldspar. Some bioclasts have } \\
\text { micritic rims. } \\
\text { Heavy-mineral concentrate includes phosphatized rock } \\
\text { fragments and bioclasts (chiefly pelmatozoan } \\
\text { ossicles, and foraminiferan and bryozoan fragments) } \\
\text { and minor fluorite. } \\
10.9 \mathrm{~kg} \text { of rock was processed. }\end{array}$ \\
\hline $\begin{array}{l}73 \\
\text { EMA } \\
\text { (Wolverine } \\
\text { Creek plate; } \\
\text { Rok window) }\end{array}$ & \begin{tabular}{|l|} 
De Long Mtns. \\
A-2 \\
$68^{\circ} 07^{\prime} 00^{\prime \prime} /$ \\
$163^{\circ} 08^{\prime} 36^{\prime \prime}$
\end{tabular} & $\begin{array}{l}\text { Kladognathus } \mathrm{sp} . \\
3 \mathrm{M}, 2 \mathrm{Sa} \text {, and } 3 \mathrm{Sb}-\mathrm{Sc} \text { elements } \\
\text { Synclydognathus geminus (Hinde) } \\
2 \mathrm{~Pa}, 2 \mathrm{~Pb} \text {, and } 1 \mathrm{~S} \text { elements } \\
\text { Unassigned elements: } \\
2 \mathrm{~Pb}, 1 \mathrm{M} \text {, and } 3 \mathrm{Sc} \text { (3 morphotypes) } \\
20 \text { indet. bar, blade, and platform fragments } \\
\mathrm{CAI}=3 \\
{[\mathrm{KE} 98-25 \mathrm{C} ; 33605-\mathrm{PC}]}\end{array}$ & $\begin{array}{l}\text { late Early Mississippian } \\
\text { (Osagean). }\end{array}$ & $\begin{array}{l}\text { Indeterminate (too few } \\
\text { generically } \\
\text { identifiable } \\
\text { conodonts); } \\
\text { postmortem transport } \\
\text { within a shallow- } \\
\text { water depositional } \\
\text { setting. }\end{array}$ & $\begin{array}{l}\text { Upper Utukok Formation. See table } 6 \text { for Kogruk } \\
\text { Formation samples from this location. } \\
\text { Light-gray-weathering, medium-dark-gray, very fine } \\
\text { grained limestone with rare coarse crinoid ossicles. } \\
\text { Thin section is crinoid-bryozoan packstone with a } \\
\text { dolomitized micritic matrix; other bioclasts are } \\
\text { brachiopods, ostracodes, calcareous and siliceous } \\
\text { spicules, and calcispheres. } \\
\text { Heavy-mineral concentrate contains ichthyoliths. } \\
4.2 \mathrm{~kg} \text { of rock was processed. } \\
\text { Loc. } 14 \text { (fig. 2) of Dumoulin and others (2004). }\end{array}$ \\
\hline $\begin{array}{l}74 \\
\text { EMA } \\
\text { (Wolverine } \\
\text { Creek plate; } \\
\text { Rok window) }\end{array}$ & $\begin{array}{l}\text { De Long Mtns. } \\
\text { A-2 } \\
68^{\circ} 066^{\prime} 56.8^{\prime \prime} / \\
163^{\circ} 07^{\prime} 20.8^{\prime \prime}\end{array}$ & $\begin{array}{l}14 \text { indet. small bar and blade fragments } \\
5 \text { ichthyoliths } \\
\text { CAI=3.5-4 } \\
{[00 \mathrm{AD} 3 \mathrm{~A} ; 33624-\mathrm{PC}]}\end{array}$ & $\begin{array}{l}\text { Mississippian on the basis of } \\
\text { stratigraphic position and } \\
\text { stratigraphic unit. }\end{array}$ & $\begin{array}{l}\text { Indeterminate (no } \\
\text { generically } \\
\text { identifiable } \\
\text { conodonts). Probably } \\
\text { a postmortem } \\
\text { winnow. }\end{array}$ & $\begin{array}{l}\text { Base of Utukok Formation or interbedded Utukok } \\
\text { Formation and Kayak Formation. } \\
\text { Grayish-red- to light-orange-weathering, medium- } \\
\text { dark-gray, fetid crinoidal supportstone with up to } \\
50 \% \text { chert and locally abundant brachiopods; beds } \\
\text { undulatory and } 3 \text { to } 7 \mathrm{~cm} \text { thick. Thin section is } \\
\text { crinoidal grainstone with lesser bryozoan and } \\
\text { brachiopod fragments, a few clay lenses } \\
\text { (intraclasts?), and traces of very fine quartz sand. } \\
\text { Heavy-mineral concentrate includes minor dolomitized } \\
\text { bryozoan fragments, indeterminate bioclasts, and } \\
\text { scarce phosphatic brachiopod fragments. } \\
8.0 \text { kg of rock was processed. }\end{array}$ \\
\hline
\end{tabular}


Table 7. Conodont samples from the Utukok Formation-Continued.

[All faunas identified by A.G. Harris. Structural units determined by L.E. Young. EMA, Endicott Mountains allochthon; KRA, Kelly River allochthon; PCA, Picnic Creek allochthon. CAI, conodont color alteration index. Letters in field number refer to collector: AD, J.A. Dumoulin; AK, J.S. Kelley; Cx, S.M. Curtis; EK, I. Ellersieck; JS, J.M. Schmidt; KE, K. Evans; K.S., K.W. Sherwood; and TR, I.L. Tailleur. DDH, diamond drill hole. All drill hole samples collected by J.A. Dumoulin. Lithologic data under remarks are field descriptions unless otherwise indicated; thin section observations by J.A. Dumoulin. No., number; indet., indeterminate; loc., locality]

\begin{tabular}{|c|c|c|c|c|c|}
\hline $\begin{array}{l}\text { Locality No., } \\
\text { structural unit }\end{array}$ & $\begin{array}{l}\text { Quadrangle, } \\
\text { latitude n./ } \\
\text { longitude w. }\end{array}$ & $\begin{array}{c}\text { Conodont fauna and CAI } \\
\text { [field No.; USGS collection No.] }\end{array}$ & Age & $\begin{array}{c}\text { Conodont biofacies and } \\
\text { depositional } \\
\text { environment }\end{array}$ & Remarks \\
\hline 79 & $\begin{array}{l}\text { De Long Mtns. } \\
\text { A-2 } \\
68^{\circ} 01^{\prime} 31.7^{\prime \prime} / \\
163^{\circ} 03^{\prime} 0.6^{\prime \prime}\end{array}$ & $\begin{array}{l}\text { Barren } \\
\text { [KS98-83] }\end{array}$ & & & $\begin{array}{l}\text { Utukok Formation(?) } \\
\text { Argillaceous or siliceous limestone and calcareous } \\
\text { siltstone. Thin section is fine-grained dolostone. } \\
5.1 \mathrm{~kg} \text { of rock was processed. } \\
\end{array}$ \\
\hline 80 & $\begin{array}{l}\text { De Long Mtns. } \\
\text { A-2 } \\
68^{\circ} 01 ' 27.8^{\prime \prime} / \\
163^{\circ} 03^{\prime} 13^{\prime \prime}\end{array}$ & $\begin{array}{l}\text { Barren } \\
\text { [KS98-82] }\end{array}$ & & & $\begin{array}{l}\text { Utukok Formation(?) } \\
\text { Moderate-yellow-brown-weathering, medium-dark- } \\
\text { gray, very fine grained limestone. Thin section is } \\
\text { micrite with scattered, abundant, very fine dolomite } \\
\text { euhedra. } \\
6.52 \mathrm{~kg} \text { of rock was processed. }\end{array}$ \\
\hline $\begin{array}{l}81 \\
\text { KRA (Wulik } \\
\text { Peaks plate) }\end{array}$ & $\begin{array}{l}\text { De Long Mtns. } \\
\text { A-2 } \\
68^{\circ} 01^{\prime} 19.2^{\prime \prime} / \\
163^{\circ} 03^{\prime} 07.2^{\prime \prime}\end{array}$ & $\begin{array}{l}\text { Barren } \\
\text { [98JS5B] }\end{array}$ & & & $\begin{array}{l}\text { Turbidites(?) in lower part of Utukok Formation. } \\
\text { Dark-gray-weathering, dark-gray limestone } \\
\text { (calcarenite) with 5\% black shale partings. Thin } \\
\text { section is deformed calcisiltite with dark (muddy?) } \\
\text { seams and minor skeletal(?) fragments. } \\
4.6 \mathrm{~kg} \text { of rock was processed. } \\
\end{array}$ \\
\hline $\begin{array}{l}83 \\
\text { EMA } \\
\text { (Wolverine } \\
\text { Creek plate; } \\
\text { Rok window) }\end{array}$ & $\begin{array}{l}\text { De Long Mtns. } \\
\text { A-2 } \\
68^{\circ} 00^{\prime} 57.5^{\prime \prime} / \\
162^{\circ} 59^{\prime} 33^{\prime \prime}\end{array}$ & $\begin{array}{l}\text { Kladognathus sp. indet. (chiefly fragments) } \\
1 \mathrm{M}, 1 \mathrm{Sa}, 3 \mathrm{Sb}-\mathrm{Sc} \text {, and } 8 \text { small bar fragments } \\
5 \text { ichthyoliths } \\
\mathrm{CAI}=2 \text { or } 3 \text { (conodonts covered with mineral and } \\
\text { organic matter). } \\
\text { [98AD14G; } 33419-\mathrm{PC}]\end{array}$ & $\begin{array}{l}\text { Mississippian but not very } \\
\text { earliest Mississippian } \\
\text { (middle Kinderhookian- } \\
\text { Chesterian) }\end{array}$ & $\begin{array}{l}\text { Indeterminate (too few } \\
\text { conodonts). } \\
\text { Conodonts present } \\
\text { suggest shallow-water } \\
\text { depositional setting. }\end{array}$ & $\begin{array}{l}\text { Utukok Formation; stratigraphically underlies Kogruk } \\
\text { Formation sampled as 98AD15A (loc. 84, table 6). } \\
\text { Dark-yellow-orange skeletal supportstone (with } \\
\text { bryozoans, crinoids, corals, brachiopods) in irregular } \\
\text { nodular beds as much as } 10 \mathrm{~cm} \text { thick, interbedded } \\
\text { with thinner bedded sandy limestone; sample is from } \\
\text { middle of } 20 \text {-m-thick section. Thin section is skeletal } \\
\text { wackestone to packstone, made up of large crinoid } \\
\text { ossicles (to } 7 \mathrm{~mm} \text { ) and smaller bryozoan and } \\
\text { ostracode? fragments in a matrix of dolomite } \\
\text { rhombs, brown noncalcareous mud, and local calcite } \\
\text { cement. } \\
\text { Heavy-mineral concentrate includes phosphatized rock } \\
\text { fragments and phosphatized bioclasts (chiefly } \\
\text { phosphatic brachiopods and ichthyoliths). } \\
5.7 \text { kg of rock was processed. }\end{array}$ \\
\hline
\end{tabular}


Table 7. Conodont samples from the Utukok Formation-Continued.

[All faunas identified by A.G. Harris. Structural units determined by L.E. Young. EMA, Endicott Mountains allochthon; KRA, Kelly River allochthon; PCA, Picnic Creek allochthon. CAI, conodont color alteration index. Letters in field number refer to collector: AD, J.A. Dumoulin; AK, J.S. Kelley; Cx, S.M. Curtis; EK, I. Ellersieck; JS, J.M. Schmidt; KE, K. Evans; K.S., K.W. Sherwood; and TR, I.L. Tailleur. DDH, diamond drill hole. All drill hole samples collected by J.A. Dumoulin. Lithologic data under remarks are field descriptions unless otherwise indicated; thin section observations by J.A. Dumoulin. No., number; indet., indeterminate; loc., locality]

\begin{tabular}{|c|c|c|c|c|c|}
\hline $\begin{array}{l}\text { Locality No., } \\
\text { structural unit }\end{array}$ & $\begin{array}{l}\text { Quadrangle, } \\
\text { latitude n./ } \\
\text { longitude w. }\end{array}$ & $\begin{array}{c}\text { Conodont fauna and CAI } \\
\text { [field No.; USGS collection No.] }\end{array}$ & Age & $\begin{array}{c}\text { Conodont biofacies and } \\
\text { depositional } \\
\text { environment }\end{array}$ & Remarks \\
\hline $\begin{array}{l}102 \\
\text { KRA (Kelly } \\
\text { plate) }\end{array}$ & \begin{tabular}{|l} 
De Long Mtns. \\
B-1 \\
$68^{\circ} 20^{\prime} 33.8^{\prime \prime} /$ \\
$162^{\circ} 12^{\prime} 47^{\prime \prime}$
\end{tabular} & $\begin{array}{l}1 \text { Pa Gnathodus cuneiformis Mehl and Thomas [pl. } \\
\text { 2, fig. 16] } \\
1 \text { Pa element fragment Hindeodus sp. indet., or } \\
\text { Synclydognathus sp. indet. } \\
1 \text { Pa element fragment Polygnathus sp. indet. or } \\
\text { Pseudopolygnathus sp. indet. } \\
\text { Unassigned elements: } \\
2 \mathrm{M}, 1 \mathrm{Sb} \text {, and } 1 \mathrm{Sc} \\
17 \text { indet. bar, blade, and platform fragments } \\
\text { CAI=2.5 } \\
{[00 \mathrm{AD} 15 \mathrm{~A} ; 33630-\mathrm{PC}]}\end{array}$ & $\begin{array}{l}\text { late Early Mississippian } \\
\text { (lower half of the Osagean; } \\
\text { Lower Gn. typicus } \\
\text { Subzone-Sc. anchoralis-Do. } \\
\text { latus Zone). }\end{array}$ & $\begin{array}{l}\text { Indeterminate (too few } \\
\text { conodonts); conodonts } \\
\text { present indicate } \\
\text { normal-marine } \\
\text { depositional setting. }\end{array}$ & $\begin{array}{l}\text { Five slabs of quartzose limestone and calcareous } \\
\text { sandstone collected from talus train derived from } \\
\text { outcrop source a few hundred meters up valley. } \\
\text { Samples are planar- to crudely-bedded, parallel- and } \\
\text { cross-bedded, grayish-orange- and medium-light- } \\
\text { gray-weathering, medium-gray, quartzose } \\
\text { calcarenite to calcareous sandstone containing } \\
\text { abundant brachiopods (mostly spiriferids) and minor } \\
\text { pelmatozoan ossicles. Strata separated by about } 75 \text { to } \\
100 \mathrm{ft} \text { of dark shale from underlying Devonian } \\
\text { carbonate rocks. Thin-section is skeletal grainstone } \\
\text { with 10-20\% ooids and 5-30\% noncarbonate sand } \\
\text { (mainly quartz, some plagioclase feldspar). Bioclasts } \\
\text { chiefly crinoid ossicles, lesser foraminifers and } \\
\text { brachiopod fragments; some bioclasts micritized. } \\
\text { Heavy-mineral concentrate includes pyritic, silicified, } \\
\text { phosphatic brachiopod fragments, and ichthyoliths. } \\
10.25 \text { kg of rock was processed. } \\
\text { Loc. } 16 \text { (app. 1) of Dumoulin and others (2004). }\end{array}$ \\
\hline $\begin{array}{l}108 \\
\text { KRA }\end{array}$ & $\begin{array}{l}\text { De Long Mtns. } \\
\text { A-1 } \\
68^{\circ} 14^{\prime} 54^{\prime \prime} / \\
162^{\circ} 14^{\prime} 30^{\prime \prime}\end{array}$ & $\begin{array}{l}1 \text { mid Pa element fragment Bispathodus stabilis } \\
\text { (Branson and Mehl) or Bi. utahensis Sandberg } \\
\text { and Gutschick } \\
2 \text { Pa elements Polygnathus communis Branson and } \\
\text { Mehl } \\
1 \text { unassigned M element } \\
\text { CAI=3-3.5 } \\
\text { [97AK65C] }\end{array}$ & $\begin{array}{l}\text { very late Late Devonian-Early } \\
\text { Mississippian (late } \\
\text { Famennian-latest Osagean). }\end{array}$ & Indeterminate. & $\begin{array}{l}\text { Sandstone at base of Utukok Formation, overlying } \\
\text { Devonian carbonate rocks (see table } 12 \text { for samples } \\
\text { from these rocks). } \\
\text { Yellow-gray-weathering, light-medium-gray, fine- } \\
\text { grained, well-sorted calcareous quartz sandstone } \\
\text { with brachiopod(?) molds. Thin section is fairly well } \\
\text { sorted, very fine grained quartz sandstone with } \\
\text { patchy (trace-15\%) calcite cement (and grains?) and } \\
\text { minor dolomite. Clasts are } 75 \% \text { monocrystalline } \\
\text { quartz and 5-10\% chert, with trace polycrystalline } \\
\text { quartz, metamorphic lithic clasts, and crinoid } \\
\text { ossicles (to } 1.5 \mathrm{~mm} \text { ). } \\
\text { Heavy-mineral concentrate includes phosphatized } \\
\text { bioclasts. } \\
7.3 \text { kg of rock was processed. }\end{array}$ \\
\hline
\end{tabular}


Table 7. Conodont samples from the Utukok Formation-Continued.

[All faunas identified by A.G. Harris. Structural units determined by L.E. Young. EMA, Endicott Mountains allochthon; KRA, Kelly River allochthon; PCA, Picnic Creek allochthon. CAI, conodont color alteration index. Letters in field number refer to collector: AD, J.A. Dumoulin; AK, J.S. Kelley; Cx, S.M. Curtis; EK, I. Ellersieck; JS, J.M. Schmidt; KE, K. Evans; K.S., K.W. Sherwood; and TR, I.L. Tailleur. DDH, diamond drill hole. All drill hole samples collected by J.A. Dumoulin. Lithologic data under remarks are field descriptions unless otherwise indicated; thin section observations by J.A. Dumoulin. No., number; indet., indeterminate; loc., locality]

\begin{tabular}{|c|c|c|c|c|c|}
\hline $\begin{array}{l}\text { Locality No., } \\
\text { structural unit }\end{array}$ & $\begin{array}{l}\text { Quadrangle, } \\
\text { latitude } \mathrm{n} . / \\
\text { longitude w. }\end{array}$ & $\begin{array}{c}\text { Conodont fauna and CAI } \\
\text { [field No.; USGS collection No.] }\end{array}$ & Age & \begin{tabular}{|c|}
$\begin{array}{c}\text { Conodont biofacies and } \\
\text { depositional } \\
\text { environment }\end{array}$ \\
\end{tabular} & Remarks \\
\hline $\begin{array}{l}108 \\
\text { KRA } \\
\text { [cont.] }\end{array}$ & $\begin{array}{l}\text { De Long Mtns. } \\
\text { A-1 } \\
68^{\circ} 14^{\prime} 54^{\prime \prime} / \\
162^{\circ} 14^{\prime} 30^{\prime \prime}\end{array}$ & $\begin{array}{l}1 \text { Pa element fragment Polygnathus sp. indet. } \\
\text { Synclydognathus sp. indet. } \\
1 \mathrm{~Pa} \text { (fragment) and } 2 \mathrm{~S} \text { elements } \\
3 \text { digyrate element fragments }(1 \mathrm{~Pa}-\mathrm{Pb} \text { and } 2 \mathrm{Sc}) \\
81 \text { indet. bar, blade, and platform fragments } \\
\text { CAI=3.5 } \\
{[97 \mathrm{AK} 66 ; 33396-\mathrm{PC}]}\end{array}$ & $\begin{array}{l}\text { probably late Early } \\
\text { Mississippian (middle } \\
\text { Kinderhookian-Osagean, } \\
\text { probably Osagean). }\end{array}$ & $\begin{array}{l}\text { Indeterminate (too few } \\
\text { conodonts). Large } \\
\text { number of conodont } \\
\text { fragments indicates } \\
\text { proximity to a high- } \\
\text { energy environment. }\end{array}$ & $\begin{array}{l}\text { Utukok Formation, 70 feet above basal sandstone } \\
\text { (97AK65C). } \\
\text { Thin section is diverse skeletal grainstone with locally } \\
\text { abundant silt to very fine sand that is mostly } \\
\text { monocrystalline quartz, rare polycrystalline quartz } \\
\text { and feldspar(?). Main bioclast is bladed algae(?) (2-4 } \\
\text { mm by } 0.5-0.75 \mathrm{~mm} \text { ); blades were probably } \\
\text { originally aragonite, now calcite spar with micritic } \\
\text { rims. Other clasts include ostracodes, crinoids, } \\
\text { bryozoans, various small algae(?), and trace } \\
\text { ooids(?). } \\
\text { Heavy-mineral concentrate includes phosphatized and } \\
\text { pyritized bioclasts (ostracodes and phosphatic } \\
\text { brachiopod fragments). } \\
6.3 \text { kg of rock was processed. } \\
\end{array}$ \\
\hline $\begin{array}{l}111 \\
\text { KRA }\end{array}$ & $\begin{array}{l}\text { De Long Mtns. } \\
\text { A-1 } \\
68^{\circ} 11^{\prime} 41.8^{\prime \prime /} \\
162^{\circ} 27^{\prime} 22^{\prime \prime}\end{array}$ & $\begin{array}{l}\text { Barren } \\
\text { [97AK23B] }\end{array}$ & & & $\begin{array}{l}\text { Utukok Formation in fault contact above Kogruk } \\
\text { Formation; see table } 6 \text { for Kogruk sample from this } \\
\text { locality. } \\
\text { Light-brown- to dark-yellow-orange-weathering, } \\
\text { medium-gray to light-brownish gray recrystallized } \\
\text { limestone that contains brachiopods and parallel- and } \\
\text { cross-laminae. Thin section is tectonized and } \\
\text { deformed, very fine grained sandstone with rare } \\
\text { bioclasts. Abundant seams, fractures, and veins. } \\
\text { Sandstone grains mostly monocrystalline quartz; } \\
\text { many grains contain fractures filled with white mica. } \\
\text { Tiny flecks of white mica throughout slide. A few } \\
\text { possible crinoid ossicles; most calcite is sparry + } \\
\text { anhedral. } \\
6.5 \text { kg of rock was processed. }\end{array}$ \\
\hline $\begin{array}{ll}113 \\
\text { KRA }\end{array}$ & $\begin{array}{l}\text { De Long Mtns. } \\
\text { A-1 } \\
68^{\circ} 12^{\prime} 00^{\prime \prime} / \\
162^{\circ} 26^{\prime} 12^{\prime \prime}\end{array}$ & $\begin{array}{l}\text { Barren } \\
{[97 \mathrm{AK} 46 \mathrm{~B}]}\end{array}$ & & & $\begin{array}{l}\text { Utukok Formation, } 15 \text { feet below contact with } \\
\text { overlying Kogruk Formation; see table } 6 \text { for Kogruk } \\
\text { sample from this locality. } \\
\text { Gray-brown- and yellow-gray-weathering, light- } \\
\text { medium-gray, fossiliferous sandy limestone. Thin } \\
\text { section is very fine grained quartz sandstone with } \\
\text { silica cement, cross-cut by calcite veins. Grains } \\
\text { mostly ( } 80 \%+\text { ) monocrystalline quartz, also chert, } \\
\text { carbonate, and trace plagioclase and tourmaline. } \\
7.0 \text { kg of rock was processed. }\end{array}$ \\
\hline
\end{tabular}


Table 7. Conodont samples from the Utukok Formation-Continued.

[All faunas identified by A.G. Harris. Structural units determined by L.E. Young. EMA, Endicott Mountains allochthon; KRA, Kelly River allochthon; PCA, Picnic Creek allochthon. CAI, conodont color alteration index. Letters in field number refer to collector: AD, J.A. Dumoulin; AK, J.S. Kelley; Cx, S.M. Curtis; EK, I. Ellersieck; JS, J.M. Schmidt; KE, K. Evans; K.S., K.W. Sherwood; and TR, I.L. Tailleur. DDH, diamond drill hole. All drill hole samples collected by J.A. Dumoulin. Lithologic data under remarks are field descriptions unless otherwise indicated; thin section observations by J.A. Dumoulin. No., number; indet., indeterminate; loc., locality]

\begin{tabular}{|c|c|c|c|c|c|}
\hline $\begin{array}{l}\text { Locality No., } \\
\text { structural unit }\end{array}$ & $\begin{array}{l}\text { Quadrangle, } \\
\text { latitude n./ } \\
\text { longitude w. }\end{array}$ & $\begin{array}{c}\text { Conodont fauna and CAI } \\
\text { [field No.; USGS collection No.] }\end{array}$ & Age & $\begin{array}{c}\text { Conodont biofacies and } \\
\text { depositional } \\
\text { environment }\end{array}$ & Remarks \\
\hline $\begin{array}{l}159 \\
\text { EMA (Key } \\
\text { Creek plate; } \\
\text { Port Road } \\
\text { succession of } \\
\text { Dumoulin and } \\
\text { others, 2004) }\end{array}$ & $\begin{array}{l}\text { Noatak D-3 } \\
67^{\circ} 51^{\prime} 04^{\prime \prime} / \\
163^{\circ} 15^{\prime} 25^{\prime \prime}\end{array}$ & $\begin{array}{l}1 \text { Pa element fragment Bispathodus sp. (no } \\
\text { accessory denticles) } \\
1 \text { Pa element Hindeodus? sp. indet. } \\
1 \text { unassigned Pb element } \\
1 \text { Sb-Sc element Kladognathus sp. indet. } \\
\text { CAI=3 } \\
\text { [DDH 1103, composite conodont sample 2: 674.5- } \\
\text { 75.5, 676-76.3, 687-87.5, 688-90, and 690.5-91 } \\
\text { ft; 33659-PC] }\end{array}$ & $\begin{array}{l}\text { late Early to middle Late } \\
\text { Mississippian (Osagean- } \\
\text { early Chesterian). Likely } P \text {. } \\
\text { mehli-Lower G. texanus } \\
\text { Zone (late Osagean) on the } \\
\text { basis of other conodont } \\
\text { collections from the top of } \\
\text { the Utukok Formation in } \\
\text { the Red Dog mine area. }\end{array}$ & $\begin{array}{l}\text { Indeterminate (too few } \\
\text { conodonts); } \\
\text { postmortem transport } \\
\text { from or within a high- } \\
\text { energy depositional } \\
\text { setting. }\end{array}$ & $\begin{array}{l}\text { Uppermost Utukok Formation. See tables } 6 \text { and } 11 \text { for } \\
\text { additional samples from this drill hole. } \\
674.5-691 \mathrm{ft} \text { : Very light- to medium-gray (outer), } \\
\text { medium-light- to medium-dark-gray (inner) } \\
\text { limestone. Several interbeds of medium-dark-gray to } \\
\text { black, noncalcareous shale as much as } 3 \mathrm{ft} \text { thick and } \\
\sim 5 \% \text { dark-brownish-gray shaly partings ( } 2 \mathrm{~mm}-3.5 \\
\mathrm{~cm} \text { thick); local layers ( } 1-30 \mathrm{~cm} \text { thick) of chert and } \\
\text { partly silicified limestone (shale, chert, and silicified } \\
\text { limestone excluded from conodont sample). } \\
\text { Limestone is bioclastic pack/wackestone with } \\
\text { crinoid ossicles to } 1.5 \mathrm{~cm} \text {. } \\
\text { Thin section data: } \\
679 \mathrm{ft} \text { : Crinoid pack/wackestone with crinoid ossicles } \\
\text { to } 1.2 \mathrm{~cm} \text {, lesser siliceous spicules, and abundant } \\
\text { stylolites. Matrix and bioclasts replaced by chert; } \\
\text { local dolomite rhombs in matrix. } \\
687.5 \mathrm{ft} \text { : Skeletal supportstone with crinoid, bryozoan, } \\
\text { ostracode, and brachiopod fragments. } \\
\text { Heavy-mineral concentrate includes phosphatic and } \\
\text { phosphatized bioclasts (chiefly ichthyoliths) and } \\
\text { minor phosphatic brachiopod fragments. } \\
6.9 \text { kg of rock was processed. } \\
\text { Loc. } 8 \text { (app. 1) of Dumoulin and others (2004). }\end{array}$ \\
\hline
\end{tabular}


Table 7. Conodont samples from the Utukok Formation-Continued.

[All faunas identified by A.G. Harris. Structural units determined by L.E. Young. EMA, Endicott Mountains allochthon; KRA, Kelly River allochthon; PCA, Picnic Creek allochthon. CAI, conodont color alteration index. Letters in field number refer to collector: AD, J.A. Dumoulin; AK, J.S. Kelley; Cx, S.M. Curtis; EK, I. Ellersieck; JS, J.M. Schmidt; KE, K. Evans; K.S., K.W. Sherwood; and TR, I.L. Tailleur. DDH, diamond drill hole. All drill hole samples collected by J.A. Dumoulin. Lithologic data under remarks are field descriptions unless otherwise indicated; thin section observations by J.A. Dumoulin. No., number; indet., indeterminate; loc., locality]

\begin{tabular}{|c|c|c|c|c|c|}
\hline $\begin{array}{l}\text { Locality No., } \\
\text { structural unit }\end{array}$ & $\begin{array}{l}\text { Quadrangle, } \\
\text { latitude } \mathrm{n} . / \\
\text { longitude w. }\end{array}$ & $\begin{array}{c}\text { Conodont fauna and CAI } \\
\text { [field No.; USGS collection No.] }\end{array}$ & Age & \begin{tabular}{|c|}
$\begin{array}{c}\text { Conodont biofacies and } \\
\text { depositional } \\
\text { environment }\end{array}$ \\
\end{tabular} & Remarks \\
\hline $\begin{array}{l}159 \\
\text { EMA (Key } \\
\text { Creek plate; } \\
\text { Port Road } \\
\text { succession of } \\
\text { Dumoulin and } \\
\text { others, 2004) } \\
\text { [cont.] }\end{array}$ & $\begin{array}{l}\text { Noatak D-3 } \\
67^{\circ} 51^{\prime} 04^{\prime \prime} / \\
163^{\circ} 15^{\prime} 25^{\prime \prime}\end{array}$ & $\begin{array}{l}1 \mathrm{P} \text { element of Eotaphrus burlingtonensis Pierce } \\
\text { and Langenheim } \\
\text { Kladognathus sp. indet. } \\
1 \mathrm{M}, 1 \mathrm{Sa} \text {, and } 6 \mathrm{Sb}-\mathrm{Sc} \text { elements, and } 1 \text { fragment } \\
32 \text { indet. bar, blade, and platform fragments } \\
\text { CAI=3 } \\
\text { [DDH 1103, composite conodont sample } 3: 877-80 \text {, } \\
881-84,884.4-85.5,886-886.3 \mathrm{ft} ; 33660-\mathrm{PC}]\end{array}$ & $\begin{array}{l}\text { late Early Mississippian } \\
\text { (middle into late Osagean, } \\
\text { Sc. anchoralis-Do. latus } \\
\text { Zone into lower part of Po. } \\
\text { mehli-Lower G. texanus } \\
\text { Zone); this collection } \\
\text { indicates that at least part of } \\
\text { the upper Utukok does not } \\
\text { extend beyond the middle } \\
\text { part of the mehli-texanus } \\
\text { Zone. }\end{array}$ & $\begin{array}{l}\text { Indeterminate (too few } \\
\text { conodonts). The } \\
\text { taphonomy of these } \\
\text { conodonts indicates } \\
\text { postmortem transport } \\
\text { within or from a high- } \\
\text { energy, shallow-water } \\
\text { depositional setting. }\end{array}$ & $\begin{array}{l}\text { Upper Utukok Formation. Beds fairly steeply dipping; } \\
\sim 110 \mathrm{ft} \text { of actual stratigraphic thickness estimated } \\
\text { between this sample and sample } 2 \text {; total true } \\
\text { stratigraphic thickness of Utukok here probably } \\
\sim 300 \mathrm{ft} \text {. Depositional contact with Kayak Shale at } \\
1272 \mathrm{ft} . \\
877-886.3 \mathrm{ft} . \text { : Very light gray (outer), medium- to } \\
\text { medium-dark-gray (inner), fine- to coarse-grained } \\
\text { bioclastic limestone; mainly crinoidal supportstone } \\
\text { with local stylolites and sparry calcite veins. } \\
\text { Thin section data: } \\
879 \mathrm{ft} \text { : Extensively dolomitized crinoidal limestone, } \\
\text { with bryozoan and brachiopod fragments and rare } \\
\text { phosphatic bioclasts. } \\
885 \mathrm{ft} \text {. Crinoid grainstone with lesser bryozoan and } \\
\text { brachiopod fragments and articulated ostracodes. } \\
\text { Heavy-mineral concentrate includes phosphatized and } \\
\text { phosphatic bioclasts, chiefly ichthyoliths and minor } \\
\text { phosphatic brachiopod fragments. } \\
7.0 \text { kg of rock was processed. }\end{array}$ \\
\hline \begin{tabular}{|l}
167 \\
KRA \\
(Eli plate)
\end{tabular} & $\begin{array}{l}\text { Noatak D-5 } \\
67^{\circ} 50^{\prime} 24^{\prime \prime} / \\
163^{\circ} 20^{\prime} 42^{\prime \prime}\end{array}$ & $\begin{array}{l}2 \text { incomplete Pa elements Bispathodus aculeatus } \\
\text { (Branson and Mehl) } \\
2 \text { incomplete Pa elements Bispathodus stabilis } \\
\text { (Branson and Mehl)? } \\
1 \text { Pa element Clydognathus cavusformis Rhodes, } \\
\text { Austin, and Druce } \\
8 \text { subadult to adult Pa elements (mostly } \\
\text { incomplete) Patrognathus variabilis Rhodes, } \\
\text { Austin and Druce } \\
2 \text { Pa elements Polygnathus communis Branson and } \\
\text { Mehl } \\
1 \text { juvenile Pa element Siphonodella sp. of middle- } \\
\text { late Kinderhookian morphotype [pl. 2, figs. 18, } \\
\text { 19] } \\
57 \text { small indet. bar, blade, and platform elements } \\
\text { CAI=1.5 } \\
\text { [81EK69C; 28583-PC] }\end{array}$ & $\begin{array}{l}\text { early Early Mississippian } \\
\text { (middle-late } \\
\text { Kinderhookian). }\end{array}$ & $\begin{array}{l}\text { High-energy, shallow- } \\
\text { water depositional } \\
\text { regime. }\end{array}$ & $\begin{array}{l}\text { Sandy limestone bed in quartzite. } \\
\text { This conodont fauna is similar to those from the Isikut } \\
\text { member of the Kayak Shale (Mull and others, 1997). } \\
\text { Fossil loc. 1, Mayfield and others (1987). }\end{array}$ \\
\hline
\end{tabular}


Table 8. Conodont samples from unnamed strata that may correlate with the Utukok Formation

[All faunas identified by A.G. Harris. Structural units determined by L.E. Young. EMA, Endicott Mountains allochthon; KRA, Kelly River allochthon. CAI, conodont color alteration index. Letters in field number refer to collector: AD, J.A. Dumoulin. Lithologic data under remarks are field descriptions unless otherwise indicated; thin section observations by J.A. Dumoulin. No., number; indet., indeterminate; loc., locality]

\begin{tabular}{|c|c|c|c|c|c|}
\hline $\begin{array}{l}\text { Locality No., } \\
\text { structural unit }\end{array}$ & $\begin{array}{l}\text { Quadrangle, } \\
\text { latitude n./ } \\
\text { longitude w. }\end{array}$ & $\begin{array}{c}\text { Conodont fauna and CAI } \\
\text { [field No.; USGS collection No.] }\end{array}$ & Age & $\begin{array}{c}\text { Conodont biofacies and } \\
\text { depositional } \\
\text { environment }\end{array}$ & Remarks \\
\hline $\begin{array}{l}95 \\
\text { KRA } \\
\text { (Amphitheatre } \\
\text { plate) }\end{array}$ & $\begin{array}{l}\text { De Long Mtns. } \\
\text { B-1 } \\
68^{\circ} 20^{\prime} 31^{\prime \prime} / \\
162^{\circ} 27^{\prime} 25.2^{\prime \prime}\end{array}$ & $\begin{array}{l}3 \text { incomplete Pa elements Bispathodus utahensis } \\
\text { Sandberg and Gutschick } \\
\text { Kladognathus sp. } \\
1 \mathrm{~Pa}-\mathrm{Pb}, 1 \mathrm{Sa} \text {, and } 3 \mathrm{Sc} \text { elements } \\
\text { Unassigned elements: } \\
1 \mathrm{~Pb}, 2 \mathrm{M} \text { ( } 2 \text { morphotypes), } 2 \mathrm{Sb} \text { ( } 2 \text { morphotypes), } \\
\text { and } 1 \mathrm{Sc} \\
29 \text { indet. bar, blade, and platform fragments } \\
\mathrm{CAI}=2.5-3.0 \\
{[04 \mathrm{AD} 40 \mathrm{H} ; 33785-\mathrm{PC}]}\end{array}$ & $\begin{array}{l}\text { middle Early-middle Late } \\
\text { Mississippian (latest } \\
\text { Kinderhookian-earliest } \\
\text { Chesterian; upper part Upper } \\
\text { Si. Isosticha-Si. crenulata } \\
\text { Zone through lower part } G n \text {. } \\
\text { bilineatus-Upper } \\
\text { Cavusgnathus Zone). }\end{array}$ & $\begin{array}{l}\text { Indeterminate (too few } \\
\text { identifiable } \\
\text { conodonts). Nearly all } \\
\text { conodonts in this } \\
\text { meager collection are } \\
\text { relatively tiny and } \\
\text { incomplete, indicating } \\
\text { postmortem transport, } \\
\text { likely from a shallow- } \\
\text { water depositional } \\
\text { setting. }\end{array}$ & $\begin{array}{l}\text { Sample from at or just above transitional top of map } \\
\text { unit } \mathrm{Mls}_{3} \text { (shale and micritc limestone member) into } \\
\mathrm{Mml}_{3} \text { (micritic limestone unit) of Lisburne Group } \\
\text { (Curtis and others, 1990). Sample taken } \sim 60 \text { to } 70 \mathrm{~m} \\
\text { above exposed base of Lisburne Group here. These } \\
\text { rocks are equivalent to the middle part of the Utukok } \\
\text { Formation in other plates of the KRA. } \\
\text { Sample from 15- to } 25 \text {-cm-thick turbidite bed within a } \\
\text { sequence of sooty black shale and fine-grained } \\
\text { limestone. Turbidite consists of pyritic limestone } \\
\text { (crinoid packstone) with muddier lenses and clasts (to } \\
25 \text { cm long) of laminated mudstone/siltstone. } \\
\text { Heavy-mineral concentrate consists chiefly of } \\
\text { composite bioclastic carbonate-pyrite grains, lesser } \\
\text { pyritic phosphatic and pyritic bioclastic carbonate } \\
\text { grains, and minor phosphatized and phosphatic } \\
\text { bioclasts (mainly bryozoan fragments, lesser } \\
\text { ostracode valves, phosphatic brachiopod fragments, } \\
\text { and rare ichthyoliths and conodonts). } \\
6.0 \text { kg of rock was processed. }\end{array}$ \\
\hline $\begin{array}{l}96 \\
\text { KRA } \\
\text { (Amphitheatre } \\
\text { plate) }\end{array}$ & $\begin{array}{l}\text { De Long Mtns. } \\
\text { B-1 } \\
68^{\circ} 19^{\prime} 26^{\prime \prime} / \\
162^{\circ} 28^{\prime} 30^{\prime \prime}\end{array}$ & $\begin{array}{l}\text { Barren. Not surprising as } 65 \% \text { of the rock did not } \\
\text { dissolve. } \\
\text { [00AD8A] }\end{array}$ & & & $\begin{array}{l}\text { Near structural base of map unit } \mathrm{Mml}_{3} \text { (micritic } \\
\text { limestone unit) of Lisburne Group (Curtis and others, } \\
\text { 1990). } \\
\text { Medium-olive-gray-weathering, moderately } \\
\text { bioturbated mudstone and lesser wackestone(?) in } \\
\text { beds } 30 \text { - to } 80 \text {-cm-thick with some } 5 \text {-cm-thick } \\
\text { intervals of platy beds. Outcrop } 3 \text { to } 5 \text { m thick. Thin } \\
\text { section is bioturbated calcareous spiculite. } \\
10.7 \mathrm{~kg} \text { of rock was processed. } \\
\text { Loc. } 24 \text { (fig. 2) of Dumoulin and others (2004). }\end{array}$ \\
\hline $\begin{array}{l}98 \\
\text { KRA } \\
\text { (Amphitheatre } \\
\text { plate) }\end{array}$ & $\begin{array}{l}\text { De Long Mtns. } \\
\text { B-1 } \\
68^{\circ} 19^{\prime} 47.7^{\prime \prime} / \\
162^{\circ} 19^{\prime} 19.4^{\prime \prime}\end{array}$ & $\begin{array}{l}\text { Barren } \\
{[00 \mathrm{AD} 12 \mathrm{~A}]}\end{array}$ & & & $\begin{array}{l}\text { Base of map unit } \mathrm{Mml}_{3} \text { (micritic limestone unit) of } \\
\text { Lisburne Group (Curtis and others, 1990) in same } \\
\text { section as 00AD9A and 10A (loc. 99). } \\
\text { Stratigraphically overlies Devonian carbonate rocks; } \\
\text { see table } 12 \text { for additional sample from this locality. } \\
\text { Light- to medium-light-gray and grayish-orange- } \\
\text { weathering, medium-dark-gray, massive- to thick- } \\
\text { bedded lime mudstone; no bioclasts noted. Thin } \\
\text { section is peloid-calcisphere supportstone. } \\
11.2 \mathrm{~kg} \text { of rock was processed. } \\
\text { Loc. } 13 \text { (app. 1) of Dumoulin and others (2004). }\end{array}$ \\
\hline
\end{tabular}


Table 8. Conodont samples from unnamed strata that may correlate with the Utukok Formation-Continued.

[All faunas identified by A.G. Harris. Structural units determined by L.E. Young. EMA, Endicott Mountains allochthon; KRA, Kelly River allochthon. CAI, conodont color alteration index. Letters in field number refer to collector: AD, J.A. Dumoulin. Lithologic data under remarks are field descriptions unless otherwise indicated; thin section observations by J.A. Dumoulin. No., number; indet., indeterminate; loc., locality]

\begin{tabular}{|c|c|c|c|c|c|}
\hline $\begin{array}{l}\text { Locality No., } \\
\text { structural unit }\end{array}$ & $\begin{array}{l}\text { Quadrangle, } \\
\text { latitude n./ } \\
\text { longitude w. }\end{array}$ & $\begin{array}{c}\text { Conodont fauna and CAI } \\
\text { [field No.; USGS collection No.] }\end{array}$ & Age & $\begin{array}{c}\text { Conodont biofacies and } \\
\text { depositional } \\
\text { environment }\end{array}$ & Remarks \\
\hline $\begin{array}{l}99 \\
\text { KRA } \\
\text { (Amphitheatre } \\
\text { plate) }\end{array}$ & $\begin{array}{l}\text { De Long Mtns. } \\
\text { B-1 } \\
68^{\circ} 19^{\prime} 22^{\prime \prime} / \\
162^{\circ} 20^{\prime} 10^{\prime \prime}\end{array}$ & $\begin{array}{l}\text { Barren } \\
{[00 \mathrm{AD} 9 \mathrm{~A}]}\end{array}$ & & & $\begin{array}{l}\text { Stratigraphic top of map unit } \mathrm{Mml}_{3} \text { (micritic limestone } \\
\text { unit) of Lisburne Group (Curtis and others, 1990). } \\
\text { Underlies Kogruk Formation; see table } 6 \text { for a } \\
\text { Kogruk sample from this locality. } \\
\text { Light-gray- to medium-brownish-gray-weathering, } \\
\text { medium-dark-gray, bryozoan mudstone with } \\
\text { fenestrate bryozoan fragments as much as } 5 \mathrm{~cm} \text { long; } \\
\text { beds as much as } 15 \text {-cm-thick at sample site, but a } \\
\text { meter lower bedding is platy and } \leq 1 \text { mm in thickness. } \\
\text { Thin section is crinoidal wackestone-mudstone with } \\
\text { bryozoan fragments and calcareous and siliceous } \\
\text { sponge spicules. } \\
8.7 \text { kg of rock was processed. } \\
\text { Loc. } 14 \text { (app. 1) of Dumoulin and others (2004). }\end{array}$ \\
\hline \multirow[t]{2}{*}{$\begin{array}{l}172 \\
\text { EMA (Key } \\
\text { Creek plate) }\end{array}$} & \multirow[t]{2}{*}{\begin{tabular}{|l|} 
Noatak D-3 \\
$67^{\circ} 50^{\prime} 31 " /$ \\
$163^{\circ} 17^{\prime} 44^{\prime \prime}$
\end{tabular}} & $\begin{array}{l}7 \text { Pa element fragments Hindeodus sp. indet. } \\
\text { Kladognathus sp. indet. } \\
6 \mathrm{M}, 2 \mathrm{Sa} \text {, and } 2 \mathrm{Sb}-\mathrm{Sc} \text { element fragments } \\
\text { Unassigned elements: } \\
2 \mathrm{M}, 2 \mathrm{Sa} \text {, and } 2 \mathrm{Sc} \text { ( } 2 \text { morphotypes), all } \\
\text { fragments } \\
107 \text { indet. bar, blade, and platform fragments } \\
\mathrm{CAI}=3 \\
{[00 \mathrm{AD} 23 \mathrm{~A} ; 33633-\mathrm{PC}]}\end{array}$ & $\begin{array}{l}\text { Early, but not earliest, } \\
\text { Mississippian (middle } \\
\text { Kinderhookian through at } \\
\text { least Osagean). }\end{array}$ & $\begin{array}{l}\text { Indeterminate (too few } \\
\text { generically identifiable } \\
\text { conodonts). } \\
\text { The preservation of the } \\
\text { conodonts (all } \\
\text { fragments) and the } \\
\text { abundant ichthyoliths } \\
\text { in this residue suggest } \\
\text { a lag concentrate. }\end{array}$ & $\begin{array}{l}\text { Unnamed limestone, } \sim 150 \mathrm{ft} \text { thick, overlying Kayak } \\
\text { Formation; similar to Utukok Formation in lithofacies } \\
\text { and fauna and provisionally included in that unit by } \\
\text { Dumoulin and others (2004). } \\
\text { Medium-light-gray- and grayish-orange-weathering, } \\
\text { light-brownish-gray, crinoidal skeletal grainstone in } \\
\text { slabby beds } 4 \text { to } 8 \mathrm{~cm} \text { thick. Thin section is crinoid- } \\
\text { bryozoan grainstone with lesser ostracode and } \\
\text { brachiopod fragments and minor dolomite. } \\
\text { Heavy-mineral concentrate includes ichthyoliths and } \\
\text { indeterminate phosphatic fragments. } \\
\text { Original sample weight not recorded. } \\
\text { Loc. } 10 \text { (app. 1) of Dumoulin and others (2004). }\end{array}$ \\
\hline & & $\begin{array}{l}1 \text { Pa fragment Eotaphrus sp. indet. } \\
1 \text { juvenile Pa element Gnathodus sp. indet. } \\
\text { Kladognathus sp. indet. } \\
16 \mathrm{M}, 1 \mathrm{Sa} \text { and } 17 \mathrm{Sb}-\mathrm{Sc} \text { element fragments } \\
\text { Synclydognathus geminus (Hinde) } \\
1 \mathrm{~Pa} \text { and } 2 \mathrm{~S} \text { and } \mathrm{M} \text { elements } \\
\text { Unassigned elements: } \\
2 \mathrm{~Pa}(2 \text { morphotypes--fragments), } 9 \mathrm{~Pb}(3 \\
\text { morphotypes), } 2 \mathrm{M} \text { ( } 2 \text { morphotypes), } 1 \mathrm{Sa} \text {, and } 2 \\
\mathrm{Sc}(2 \text { morphotypes, both fragments) } \\
144 \text { indet. bar, blade, and platform fragments } \\
\text { CAI=3 } \\
\text { [00AD23B; 33634-PC] }\end{array}$ & $\begin{array}{l}\text { late Early Mississippian } \\
\text { (Osagean, possibly middle- } \\
\text { late Osagean) }\end{array}$ & A lag concentrate. & $\begin{array}{l}\text { Same unit and structural level as } 00 \mathrm{AD} 23 \mathrm{~A} \text {, but } 40 \mathrm{ft} \\
\text { higher in section. } \\
\text { Lithofacies like } 00 \mathrm{AD} 23 \mathrm{~A} \text { but beds to } 30 \mathrm{~cm} \text { thick; } \\
\text { contains crinoid ossicles to } 0.75 \mathrm{~cm} \text { in diameter and } \\
\text { partly silicified rugose corals. Thin section is crinoid- } \\
\text { bryozoan grainstone with lesser gastropod(?) and } \\
\text { trilobite(?) fragments. } \\
\text { Heavy-mineral concentrate includes ichthyoliths and } \\
\text { indeterminate phosphatic fragments. } \\
12.9 \mathrm{~kg} \text { of rock was processed. }\end{array}$ \\
\hline
\end{tabular}


Table 9. Conodont samples from the Etivluk Group and Okpikruak Formation

[All faunas identified by A.G. Harris. Structural units determined by L.E. Young. EMA, Endicott Mountains allochthon; KRA, Kelly River allochthon. CAI, conodont color alteration index. Letters in field number refer to collector: AD, J.A. Dumoulin; JS, J.M. Schmidt; KE, K. Evans; and K. S. K.W. Sherwood. DDH, diamond drill hole. Interval thicknesses in drill holes are drilled thicknesses and have not been corrected for dip of bedding. All drill hole samples collected by J.A. Dumoulin. Lithologic data under remarks are field descriptions unless otherwise indicated; thin section observations by J.A. Dumoulin. No., number; indet., indeterminate; loc., locality]

\begin{tabular}{|c|c|c|c|c|c|}
\hline $\begin{array}{l}\text { Locality No., } \\
\text { structural unit }\end{array}$ & $\begin{array}{l}\text { Quadrangle, } \\
\text { latitude n./ } \\
\text { longitude w. }\end{array}$ & $\begin{array}{c}\text { Conodont fauna and CAI } \\
\text { [field No.; USGS collection No.] }\end{array}$ & Age & \begin{tabular}{|c|}
$\begin{array}{c}\text { Conodont biofacies and } \\
\text { depositional } \\
\text { environment }\end{array}$ \\
\end{tabular} & Remarks \\
\hline $\begin{array}{l}36 \\
\text { PCA? }\end{array}$ & $\begin{array}{l}\text { De Long Mtns. } \\
\text { B-2 } \\
68^{\circ} 21^{\prime} 46^{\prime \prime} / \\
162^{\circ} 50^{\prime} 12.7^{\prime \prime}\end{array}$ & $\begin{array}{l}36 \text { Pa element fragments Neogondolella navicula } \\
\text { (Huckriede) group } \\
1 \text { Sa element Neogondolella sp. indet. } \\
\text { CAI=2-2.5 } \\
\text { [KS98-147B; Mes. 35020] }\end{array}$ & $\begin{array}{l}\text { Middle-Late Triassic, most } \\
\text { likely middle Late Triassic. }\end{array}$ & $\begin{array}{l}\text { Postmortem transport } \\
\text { within or from the } \\
\text { neogondolellid } \\
\text { biofacies as virtually } \\
\text { all specimens are } \\
\text { platform elements; } \\
\text { slope or basin } \\
\text { depositional setting. }\end{array}$ & $\begin{array}{l}\text { Otuk Formation of Etivluk Group. } \\
\text { Buff-colored argillaceous carbonate rocks with locally } \\
\text { abundant shell fragments overlying sequence of red } \\
\text { and green cherts. Thin section is bivalve (monotid) } \\
\text { wackestone. } \\
\text { Heavy-mineral concentrate includes minor } \\
\text { phosphatized shell fragments and rare ichthyoliths. } \\
8.8 \text { kg of rock was processed. } \\
\text { Monotid bivalves and Palaeocardita(?) sp. of Late } \\
\text { Triassic age occurs in these strata (identified by R.B. } \\
\text { Blodgett, Oregon State University, unpublished fossil } \\
\text { report, 1999). }\end{array}$ \\
\hline $\begin{array}{l}62 \\
\text { KRA? }\end{array}$ & $\begin{array}{l}\text { De Long Mtns. } \\
\text { A-2 } \\
68^{\circ} 10^{\prime} 51^{\prime \prime} / \\
163^{\circ} 10^{\prime} 25.6^{\prime \prime}\end{array}$ & $\begin{array}{l}\text { Triassic conodonts: } \\
4 \text { juvenile and incomplete Pa elements } \\
\text { Neogondolella sweeti Kozur? } \\
1 \text { juvenile Pa element Neogondolella sp. indet. } \\
\text { Pennsylvanian conodonts: } \\
2 \text { juvenile Pa elements Declinognathodus } \\
\text { noduliferus (Ellison and Graves) subsp. indet. } \\
\text { (base of Pennsylvanian-early Desmoinesian) } \\
\text { Mississippian and (or) Pennsylvanian conodonts: } \\
2 \text { Pa element fragments of Cavusgnathus sp. indet. } \\
\text { or Adetognathus sp. indet. (late Meramecian- } \\
\text { Chesterian or latest Chesterian-early Early } \\
\text { Permian) } \\
1 \text { Pa element fragment Rhachistognathus sp. indet. } \\
\text { (early Chesterian-early Atokan) } \\
\text { Mississippian conodonts: } \\
2 \text { Pa element fragments Gnathodus texanus } \\
\text { Roundy? (late Osagean-earliest Chesterian) } \\
2 \text { Sb-Sc Kladognathus sp. indet. elements (middle } \\
\text { Kinderhookian-Chesterian) } \\
2 \text { incomplete S elements Synclydognathus sp. indet. } \\
\text { (Osagean-earliest Chesterian) } \\
1 \text { unassigned M element } \\
60 \text { indet. bar, blade, and platform fragments } \\
\text { CAI=2.5-3 } \\
\text { [98JS9N; Mes. 35021] }\end{array}$ & $\begin{array}{l}\text { The collection contains } \\
\text { conodonts from at least three } \\
\text { sources. These are: } \\
\text { (1) middle Early Triassic } \\
\text { (Smithian) conodonts from } \\
\text { the Etivluk Group; } \\
\text { (2) earliest Early-Middle } \\
\text { Pennsylvanian (earliest } \\
\text { Morrowan-early } \\
\text { Desmoinesian) conodonts } \\
\text { from a correlative of the } \\
\text { Wahoo Limestone of the } \\
\text { Lisburne Group (possibly } \\
\text { the Nuka Formation); and } \\
\text { (3) Mississippian conodonts } \\
\text { from the Kogruk Formation } \\
\text { and (or) related units. }\end{array}$ & $\begin{array}{l}\text { The Triassic conodonts } \\
\text { are normal-marine, } \\
\text { middle shelf to deeper } \\
\text { water forms. The } \\
\text { Pennsylvanian } \\
\text { conodonts are } \\
\text { characteristic of mainly } \\
\text { shallow-water, high- } \\
\text { energy environments. } \\
\text { The Mississippian } \\
\text { conodonts include } \\
\text { shallow and deeper } \\
\text { water shelf species. }\end{array}$ & $\begin{array}{l}\text { Boulder of heterolithic carbonate breccia within the } \\
\text { Jurassic-Cretaceous Okpikruak Formation. Main } \\
\text { lithology is ooid-skeletal packstone derived from the } \\
\text { Mississippian Kogruk Formation(?), but conodont } \\
\text { fauna indicates that younger carbonate lithologies } \\
\text { (probable Otuk Formation of the Etivluk Group and } \\
\text { possible Nuka Formation) are also present. See tables } \\
6 \text { and } 7 \text { for additional samples from this locality. } \\
\text { Boulder } 3 \mathrm{~m} \text { across of matrix-supported carbonate } \\
\text { breccia with lesser chert clasts; clasts } 20-30 \%, \\
\text { subrounded to subangular. Thin section is heterolithic } \\
\text { clastic carbonate; clasts chiefly ooid-bioclastic } \\
\text { packstone with dolomitic matrix and lesser ooid } \\
\text { grainstone. } \\
\text { Heavy-mineral concentrate is chiefly phosphatized } \\
\text { rock fragments. } \\
5.4 \text { kg of rock was processed. }\end{array}$ \\
\hline
\end{tabular}


Table 9. Conodont samples from the Etivluk Group and Okpikruak Formation - Continued.

[All faunas identified by A.G. Harris. Structural units determined by L.E. Young. EMA, Endicott Mountains allochthon; KRA, Kelly River allochthon. CAI, conodont color alteration index. Letters in field number refer to collector: AD, J.A. Dumoulin;

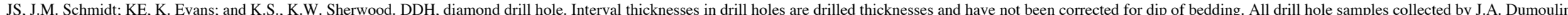
Lithologic data under remarks are field descriptions unless otherwise indicated; thin section observations by J.A. Dumoulin. No., number; indet., indeterminate; loc., locality]

\begin{tabular}{|c|c|c|c|c|c|}
\hline $\begin{array}{l}\text { Locality No., } \\
\text { structural unit }\end{array}$ & $\begin{array}{l}\text { Quadrangle, } \\
\text { latitude n./ } \\
\text { longitude w. }\end{array}$ & $\begin{array}{c}\text { Conodont fauna and CAI } \\
\text { [field No.; USGS collection No.] }\end{array}$ & Age & $\begin{array}{c}\text { Conodont biofacies and } \\
\text { depositional } \\
\text { environment }\end{array}$ & Remarks \\
\hline $\begin{array}{l}66 \\
\text { EMA (Red Dog } \\
\text { plate) }\end{array}$ & $\begin{array}{l}\text { De Long Mtns. } \\
\text { A-2 } \\
68^{\circ} 10^{\prime} 18^{\prime \prime} / \\
162^{\circ} 50^{\prime} 48^{\prime \prime}\end{array}$ & $\begin{array}{l}1 \text { Pa element Neogondolella of the Neogondolella } \\
\text { navicula Group } \\
\text { CAI=2 } \\
\text { [KE98-28; Mes. 35019] }\end{array}$ & $\begin{array}{l}\text { Conodonts indicate a late } \\
\text { Middle-Late Triassic age, } \\
\text { but radiolarians restrict age } \\
\text { to middle Late Triassic (late } \\
\text { middle-early late Norian). }\end{array}$ & $\begin{array}{l}\text { Indeterminate (too few } \\
\text { conodonts); most likely } \\
\text { normal-marine } \\
\text { depositional setting. }\end{array}$ & $\begin{array}{l}\text { Otuk Formation (Etivluk Group). See table } 14 \text { for } \\
\text { radiolarian collection from this locality. } \\
\text { Olive-gray, very fine grained dolostone(?) interbedded } \\
\text { with radiolarian chert. Carbonate beds contain rare } \\
\text { thin bivalve shells identified as Monotis(?) sp. of Late } \\
\text { Triassic age by R.B. Blodgett (Oregon State } \\
\text { University, unpublished fossil report, 1999). Thin } \\
\text { section is very fine crystalline dolomite mosaic with } \\
\text { minor intercrystalline quartz and a few thin bivalve } \\
\text { shells partly replaced by quartz and phosphate. } \\
6.0 \text { kg of rock was processed. }\end{array}$ \\
\hline $\begin{array}{l}67 \\
\text { EMA } \\
\text { (Wolverine } \\
\text { Creek plate; } \\
\text { Mt. Raven } \\
\text { window) }\end{array}$ & $\begin{array}{l}\text { De Long Mtns. } \\
\text { A-2 } \\
68^{\circ} 11^{\prime} 39^{\prime \prime} / \\
162^{\circ} 40^{\prime} 27.8^{\prime \prime}\end{array}$ & $\begin{array}{l}\text { Barren } \\
\text { [99AD14Z] }\end{array}$ & & & $\begin{array}{l}\text { Sample from basal Siksikpuk Formation depositionally } \\
\text { overlying Lisburne Group; upper Lisburne succession } \\
\text { here much like that at 99AD13 (loc. 42, table 6). } \\
\text { Sample from 10- to 20-cm-thick beds of light-gray- to } \\
\text { moderate-brown-gray-weathering, dark-gray, fine- } \\
\text { grained, peloidal(?) carbonate rock interbedded with } \\
\text { greenish-gray siltstone. Thin section is fine-grained } \\
\text { impure carbonate, possibly altered (calcitized) } \\
\text { volcanic(?) rock. } \\
8.43 \text { kg of rock was processed. } \\
\text { Loc. } 6 \text { (fig. } 2 \text { ) of Dumoulin and others (2004). } \\
\end{array}$ \\
\hline $\begin{array}{l}126 \\
\text { EMA } \\
\text { (Wolverine } \\
\text { Creek plate) }\end{array}$ & $\begin{array}{l}\text { De Long Mtns. } \\
\text { A-2 } \\
68^{\circ} 09^{\prime} 20^{\prime \prime} / \\
163^{\circ} 01^{\prime} 40^{\prime \prime}\end{array}$ & $\begin{array}{l}\text { Barren } \\
\text { [DDH 927 (west of Anarraaq deposit) composite } \\
\text { conodont sample: } 1693.2-94.2,1695-95.3,1696.5- \\
\text { 97, 1699-99.5, 1699.7-1700.7, 1701.2-01.4, } \\
\text { 1701.5-03 ft] }\end{array}$ & & & $\begin{array}{l}\text { Sample from upper subunit of Otuk Formation (Etivluk } \\
\text { Group) 72 ft below conformable contact with } \\
\text { overlying Kingak Formation(?) and } \sim 3 \mathrm{ft} \text { above } \\
\text { faulted contact with middle subunit of Otuk. See table } \\
14 \text { for radiolarian collection from this locality. } \\
1693-1703 \mathrm{ft} \text { : Very light to medium-light-gray (outer), } \\
\text { medium-gray (inner), very fine grained, locally cherty } \\
\text { carbonate with concentrations of monotid bivalves } \\
\text { and dark shaly layers a few centimeters thick (shalier } \\
\text { and chertier intervals not sampled). Thin section at } \\
1699.5 \mathrm{ft} \text { is argillaceous, dolomitic(?) micrite with } \\
\text { sparse small shell fragments, spines, and radiolarians; } \\
\text { some bioclasts partly silicified. } \\
4.5 \text { kg of rock was processed and analyzed by Andrea } \\
\text { Krumhardt, University of Alaska (Fairbanks). }\end{array}$ \\
\hline
\end{tabular}


Table 9. Conodont samples from the Etivluk Group and Okpikruak Formation - Continued.

[All faunas identified by A.G. Harris. Structural units determined by L.E. Young. EMA, Endicott Mountains allochthon; KRA, Kelly River allochthon. CAI, conodont color alteration index. Letters in field number refer to collector: AD, J.A. Dumoulin; JS, J.M. Schmidt; KE, K. Evans; and K. S. K.W. Sherwood. DDH, dimond drill hole. Interval thicknesses in drill holes are drilled thicknesses and have not been corrected for dip of bedding. All drill hole samples collected by J.A. Dumoulin. Lithologic data under remarks are field descriptions unless otherwise indicated; thin section observations by J.A. Dumoulin. No., number; indet., indeterminate; loc., locality]

\begin{tabular}{|c|c|c|c|c|c|}
\hline $\begin{array}{l}\text { Locality No., } \\
\text { structural unit }\end{array}$ & $\begin{array}{l}\text { Quadrangle, } \\
\text { latitude n./ } \\
\text { longitude w. }\end{array}$ & $\begin{array}{c}\text { Conodont fauna and CAI } \\
\text { [field No.; USGS collection No.] }\end{array}$ & Age & $\begin{array}{c}\text { Conodont biofacies and } \\
\text { depositional } \\
\text { environment }\end{array}$ & Remarks \\
\hline $\begin{array}{l}127 \\
\text { EMA } \\
\text { (Wolverine } \\
\text { Creek plate) }\end{array}$ & $\begin{array}{l}\text { De Long Mtns. } \\
\text { A-2 } \\
68^{\circ} 09^{\prime} 40^{\prime \prime} / \\
163^{\circ} 00^{\prime} 20^{\prime \prime}\end{array}$ & $\begin{array}{l}\text { Barren } \\
\text { [DDH } 1110 \text { (west of Anarraaq deposit) composite } \\
\text { conodont sample: 2080.6-82.3, 2083-83.5, 2084.7- } \\
85.2,2087-89 \mathrm{ft}]\end{array}$ & & & $\begin{array}{l}\text { Sample from upper subunit of Otuk Formation (Etivluk } \\
\text { Group) 34 ft below conformable contact with } \\
\text { overlying Kingak Formation(?). Contact with middle } \\
\text { subunit of Otuk not penetrated in this drill hole; upper } \\
\text { subunit is at least } 156 \mathrm{ft} \text { thick. } \\
2080-2089 \mathrm{ft} \text { : Light-gray (outer), medium-dark- to } \\
\text { medium-olive gray (inner), very fine grained cherty } \\
\text { carbonate with local lenses of thin shells (mainly } \\
\text { monotid bivalves) and irregular layers a few } \\
\text { centimeters thick of medium-gray radiolarian chert } \\
\text { and medium-dark-gray shale (chert and shale not } \\
\text { sampled). } \\
\text { Thin section data: } \\
2080.9,2083.5 \mathrm{ft} \text { argillaceous, cherty, dolomitic } \\
\text { micrite with locally abundant monotid bivalve } \\
\text { fragments and sparse, poorly preserved radiolarians. } \\
4.0 \text { kg of rock was processed and analyzed by Andrea } \\
\text { Krumhardt, University of Alaska (Fairbanks). }\end{array}$ \\
\hline
\end{tabular}


Table 10. Conodont samples from the Nuka Formation and related rocks

[All faunas identified by A.G. Harris. Structural units determined by L.E. Young. NRA, Nuka Ridge allochthon. CAI, conodont color alteration index. Letters in field number refer to collector: AD, J.A. Dumoulin; and Cx, S.M. Curtis. Lithologic data under remarks are field descriptions unless otherwise indicated; thin section observations by J.A. Dumoulin. No., number; indet., indeterminate; loc., locality]

\begin{tabular}{|c|c|c|c|c|c|}
\hline $\begin{array}{l}\text { Locality No., } \\
\text { structural unit }\end{array}$ & $\begin{array}{l}\text { Quadrangle, } \\
\text { latitude n./ } \\
\text { longitude w. }\end{array}$ & $\begin{array}{c}\text { Conodont fauna and CAI } \\
\text { [field No.; USGS collection No.] }\end{array}$ & Age & $\begin{array}{c}\text { Conodont biofacies and } \\
\text { depositional } \\
\text { environment }\end{array}$ & Remarks \\
\hline $\begin{array}{l}16 \\
\text { Olistolith } \\
\text { derived from } \\
\text { NRA }\end{array}$ & $\begin{array}{l}\text { De Long Mtns. } \\
\text { A-3 } \\
68^{\circ} 08^{\prime} 00^{\prime \prime} / \\
163^{\circ} 38^{\prime} 42^{\prime \prime}\end{array}$ & $\begin{array}{l}3 \text { Pa element fragments Gnathodus bilineatus } \\
\text { (Roundy) } \\
2 \text { juvenile Pa elements Gnathodus girtyi Hass subsp. } \\
\text { indet. } \\
2 \text { Pa element fragments Gnathodus spp. indet. } \\
1 \text { subadult Pa element Idiognathodus sp. } \\
1 \text { Pb element Idioprioniodus sp. } \\
21 \text { indet. bar, blade, and platform fragments } \\
\text { CAI=2.5-3 } \\
{[79 \mathrm{CX} 125 \mathrm{H}-1 ; 27552-\mathrm{PC}]}\end{array}$ & $\begin{array}{l}\text { No older than late Morrowan } \\
\text { (early Middle } \\
\text { Pennsylvanian); the fauna } \\
\text { contains mainly Chesterian } \\
\text { conodonts with an } \\
\text { Idiognathodus of no older } \\
\text { than earliest Middle } \\
\text { Pennsylvanian age. }\end{array}$ & $\begin{array}{l}\text { Indeterminate (mixed } \\
\text { age-redeposited fauna); } \\
\text { the Late Mississippian } \\
\text { and Pennsylvanian } \\
\text { conodonts are all } \\
\text { normal-marine } \\
\text { relatively shallow- } \\
\text { water forms. }\end{array}$ & $\begin{array}{l}\text { Nuka Formation. } \\
\text { This fauna resembles those collected by J. Siok in } \\
\text { the Killik River quadrangle from a 2-m-thick } \\
\text { phosphatic interval at the top of the Lisburne } \\
\text { Group. The interval contains redeposited } \\
\text { conodonts of Mississippian and Early and Middle } \\
\text { Pennsylvanian age and is no older than } \\
\text { Desmoinesian (late Middle Pennsylvanian) and no } \\
\text { younger than Leonardian (late Early Permian). } \\
\text { Fossil loc. 22, Mayfield and others (1990). }\end{array}$ \\
\hline $\begin{array}{l}118 \\
\text { NRA (Bastille } \\
\text { plate) }\end{array}$ & $\begin{array}{l}\text { Misheguk Mtn. } \\
\text { B-5 } \\
68^{\circ} 19^{\prime} 58.6^{\prime \prime} / \\
161^{\circ} 37^{\prime} 04^{\prime \prime}\end{array}$ & $\begin{array}{l}\text { Barren. No conodonts or other phosphatic or } \\
\text { phosphatized fossil materials were found. } \\
\text { [03AD34C] }\end{array}$ & & & $\begin{array}{l}\text { Mississippian and (or) Devonian limestone (map } \\
\text { unit } \mathrm{MDl}_{5} \text { ) of Curtis and others (1984). These } \\
\text { authors report Devonian corals from <0.5 mi to } \\
\text { the southwest in this unit (fossil loc. } 61 \text { ), and } \\
\text { Middle to early Late Devonian (Frasnian or older) } \\
\text { brachiopods and corals from } \sim 1 \mathrm{mi} \text { to west (fossil } \\
\text { loc. } 59 \text { ). Unit here is } \sim 80 \text { to } 100 \mathrm{~m} \text { thick and is } \\
\text { mostly dolostone with some limestone intervals. } \\
\text { Sample from } 8 \text {-cm-thick bed at base of } 30 \mathrm{~cm} \\
\text { interval of brown-gray limestone that weathers } \\
\text { very pale yellow brown. Beds thin upward to } 5 \\
\text { and then } 3 \mathrm{~cm} \text { thick. Skeletal supportstone with } \\
\text { ostracodes(?). Thin section is partly dolomitized } \\
\text { skeletal packstone; bioclasts mainly small } \\
\text { brachiopods and (or) ostracodes. } \\
6.45 \mathrm{~kg} \text { of rock was processed. }\end{array}$ \\
\hline $\begin{array}{l}119 \\
\text { NRA (Bastille } \\
\text { plate) }\end{array}$ & $\begin{array}{l}\text { Misheguk Mtn. } \\
\text { B-4 } \\
68^{\circ} 28^{\prime} 55^{\prime \prime} / \\
161^{\circ} 19^{\prime} 47^{\prime \prime}\end{array}$ & $\begin{array}{l}\text { Barren. No conodonts or other mineralized fossil } \\
\text { materials were found. } \\
{[00 \mathrm{AD} 27 \mathrm{~A}]}\end{array}$ & & & $\begin{array}{l}\text { Mississippian and (or) Devonian limestone (map } \\
\text { unit } \mathrm{MDl}_{5} \text { ) of Curtis and others (1984). Outcrop } \\
\text { on lower slopes of Copter Peak; sample } \sim 50 \text { to } 100 \\
\text { m below base of Etivluk Group. } \\
\text { Sample from } 30 x 60 \text {-cm lens of skeletal grainstone } \\
\text { within laminated carbonate sequence. Grainstone } \\
\text { is medium-dark-gray-weathering, medium-brown- } \\
\text { gray, and poorly sorted with some lithic grains } \\
\text { and mud clasts to } 1 \mathrm{~cm} \text { long. Thin section is } \\
\text { dolomitic, recrystallized(?) crinoidal supportstone. } \\
9.7 \mathrm{~kg} \text { of rock was processed. }\end{array}$ \\
\hline
\end{tabular}


Table 11. Conodont samples from the Endicott Group

[All faunas identified by A.G. Harris. Structural units determined by L.E. Young. EMA, Endicott Mountains allochthon; PCA Picnic Creek allochthon. CAI, conodont color alteration index. Letters in field number refer to collector: AD, J.A. Dumoulin; AK, J.S. Kelley; Cx, S.M. Curtis; EK, I. Ellersieck; and MD, C.F. Mayfield. DDH, diamond drill hole. Interval thicknesses in drill holes are drilled thicknesses and have not been corrected for dip of bedding. All drill hole samples collected by J.A. Dumoulin and (or) A.G. Harris. Lithologic data under remarks are field descriptions unless otherwise indicated; thin section observations by J.A. Dumoulin. No., number; indet, indeterminate; loc., locality]

\begin{tabular}{|c|c|c|c|c|c|}
\hline $\begin{array}{l}\text { Locality No., } \\
\text { structural unit }\end{array}$ & $\begin{array}{l}\text { Quadrangle, } \\
\text { latitude n./ } \\
\text { longitude w. }\end{array}$ & $\begin{array}{c}\text { Conodont fauna and CAI } \\
\text { [field No.; USGS collection No.] }\end{array}$ & Age & $\begin{array}{c}\text { Conodont biofacies and } \\
\text { depositional } \\
\text { environment }\end{array}$ & Remarks \\
\hline $\begin{array}{l}13 \\
\text { PCA (Amaruk } \\
\text { plate) }\end{array}$ & $\begin{array}{l}\text { De Long Mtns. } \\
\text { A-3 } \\
68^{\circ} 08^{\prime} 38.4^{\prime \prime} / \\
163^{\circ} 38^{\prime} 40.2^{\prime \prime}\end{array}$ & $\begin{array}{l}1 \mathrm{M} \text { element Kladognathus } \mathrm{sp} \text {. indet. } \\
1 \text { posterior Pa element fragment } \\
\text { Pseudopolygnathus } \text { sp. indet. of Mississippian } \\
\text { morphotype } \\
2 \text { indet. bar or blade fragments } \\
5 \text { ichthyoliths } \\
\text { CAI=2.5-3 } \\
\text { [79CX127G] }\end{array}$ & $\begin{array}{l}\text { Early, but not earliest, } \\
\text { Mississippian (middle } \\
\text { Kinderhookian-Osagean). }\end{array}$ & $\begin{array}{l}\text { Indeterminate (too few } \\
\text { conodonts). }\end{array}$ & $\begin{array}{l}\text { Kayak Shale. } \\
\text { Fossil loc. 18, Mayfield and others (1990). }\end{array}$ \\
\hline $\begin{array}{l}15 \\
\text { PCA (Amaruk } \\
\text { plate) }\end{array}$ & $\begin{array}{l}\text { De Long Mtns. } \\
\text { A-3 } \\
68^{\circ} 07^{\prime} 40.7^{\prime \prime} / \\
163^{\circ} 43^{\prime} 04.8^{\prime \prime}\end{array}$ & $\begin{array}{l}5 \text { robust Pa elements Bispathodus stabilis (Branson } \\
\text { and Mehl) } \\
1 \text { Sb-Sc element Kladognathus sp. indet. } \\
10 \text { Pa elements Polygnathus communis Branson } \\
\text { and Mehl } \\
1 \text { Pa element Polygnathus sp. } \\
3 \text { Pa elements Pseudopolygnathus sp. } \\
15 \text { Pa elements Siphonodella spp. of middle-late } \\
\text { Kinderhookian morphotype } \\
9 \text { Pa element large fragments Siphonodella spp. } \\
\text { Unassigned elements: } \\
1 \text { Pb, } 1 \mathrm{M}, 1 \text { Sa, } 1 \mathrm{Sb} \text {, and } 3 \mathrm{Sc} \text { (3 morphotypes) } \\
86 \text { indet. bar, blade, and platform fragments } \\
\\
\text { CAI=2.5-3 } \\
\text { [79MD171D; 27559-PC) }\end{array}$ & $\begin{array}{l}\text { early Early Mississippian } \\
\text { (middle-late } \\
\text { Kinderhookian). }\end{array}$ & $\begin{array}{l}\text { Siphonodellid- } \\
\text { polygnathid biofacies; } \\
\text { open-marine middle- } \\
\text { shelf or deeper water } \\
\text { depositional setting. } \\
\text { The size of the } \\
\text { conodonts suggests a } \\
\text { lag concentrate. }\end{array}$ & $\begin{array}{l}\text { Kayak Shale. } \\
\text { Conodont assemblage is most similar to those from the } \\
\text { Rough Mountain Creek unit of the Lisburne Group } \\
\text { (Dumoulin and Harris, 1997) and from open-marine } \\
\text { facies of the Utukok Formation. } \\
\text { Fossil loc. 31, Mayfield and others (1990). }\end{array}$ \\
\hline
\end{tabular}


Table 11. Conodont samples from the Endicott Group-Continued.

[All faunas identified by A.G. Harris. Structural units determined by L.E. Young. EMA, Endicott Mountains allochthon; PCA Picnic Creek allochthon. CAI, conodont color alteration index. Letters in field number refer to collector: AD, J.A. Dumoulin; AK, J.S. Kelley; Cx, S.M. Curtis; EK, I. Ellersieck; and MD, C.F. Mayfield. DDH, diamond drill hole. Interval thicknesses in drill holes are drilled thicknesses and have not been corrected for dip of bedding. All drill hole samples collected by J.A.
Dumoulin and (or) A.G. Harris. Lithologic data under remarks are field descriptions unless otherwise indicated; thin section observations by J.A. Dumoulin. No., number; indet., indeterminate; loc., locality]

\begin{tabular}{|c|c|c|c|c|c|}
\hline $\begin{array}{l}\text { Locality No., } \\
\text { structural unit }\end{array}$ & $\begin{array}{l}\text { Quadrangle, } \\
\text { latitude n./ } \\
\text { longitude w. }\end{array}$ & $\begin{array}{c}\text { Conodont fauna and CAI } \\
\text { [field No.; USGS collection No.] }\end{array}$ & Age & $\begin{array}{c}\text { Conodont biofacies and } \\
\text { depositional } \\
\text { environment }\end{array}$ & Remarks \\
\hline \multirow[t]{2}{*}{$\begin{array}{l}86 \\
\text { EMA } \\
\text { (Wolverine } \\
\text { Creek plate; } \\
\text { Rok window) }\end{array}$} & \multirow[t]{2}{*}{$\begin{array}{l}\text { De Long Mtns. } \\
\text { A-2 } \\
68^{\circ} 01^{\prime} 17.6^{\prime \prime} / \\
162^{\circ} 47^{\prime} 37.8^{\prime \prime}\end{array}$} & $\begin{array}{l}1 \text { unassigned } \mathrm{M} \text { element of post-Silurian } \\
\text { morphotype } \\
8 \text { indet. bar, blade, and platform fragments } \\
\text { CAI=3-3.5 } \\
\text { [97AK4] }\end{array}$ & $\begin{array}{l}\text { Conodonts in this collection } \\
\text { merely indicate a post- } \\
\text { Silurian Paleozoic age. } \\
\text { However, all other fossil } \\
\text { collections from the Kayak } \\
\text { in the western and central } \\
\text { Brooks Range that have } \\
\text { produced } \\
\text { biostratigraphically useful } \\
\text { fossils restrict the age of the } \\
\text { formation to the early Early } \\
\text { Mississippian } \\
\text { (Kinderhookian). }\end{array}$ & $\begin{array}{l}\text { Indeterminate (no } \\
\text { generically identifiable } \\
\text { conodonts). }\end{array}$ & $\begin{array}{l}\text { Upper part of Kayak Shale, from limy interval } \sim 1 \mathrm{~m} \\
\text { thick, overlying } \sim 10 \mathrm{~m} \text { of interbedded dark gray } \\
\text { argillite and yellow-green-weathering siltstone and } \\
\text { very fine grained sandstone. } \\
\text { Sample from brown-yellow-weathering, iron-stained, } \\
\text { impure fossiliferous limestone. Thin section is } \\
\text { skeletal wackestone/packstone, made up of crinoid } \\
\text { and lesser bryozoan fragments, sponge spicules } \\
\text { (calcareous and siliceous), brachiopods, ostracodes, } \\
\text { and gastropods(?). Bioclasts ( } 30-50 \% \text { of slide) } \\
\text { concentrated into vague layers; matrix is brown, } \\
\text { noncalcareous mudstone with less than } 10 \% \text { quartz } \\
\text { silt. Bioclasts abraded, some partly replaced by } \\
\text { pyrite. One rounded lithoclast ( } 8 \text { mm diameter) } \\
\text { contains abundant calcareous spicules to } 800 \mu \\
\text { (including one biaxial spicule). } \\
\text { Heavy-mineral concentrate contains phosphatic and } \\
\text { phosphatized, partly pyritized and ferruginous } \\
\text { bioclasts (including brachiopods and spine and tube } \\
\text { steinkerns) and minor euhedral pyrite. } \\
7.0 \text { kg of rock was processed. }\end{array}$ \\
\hline & & $\begin{array}{l}\text { Synclydognathus } \mathrm{n} . \mathrm{sp} \text {. } \\
13 \mathrm{~Pa}, 4 \mathrm{~Pb} \text {, and } 7 \mathrm{~S} \text { and } \mathrm{M} \text { elements } \\
33 \text { indet. bar, blade, and platform fragments } \\
\text { CAI=3-3.5 } \\
\text { [97AK5; 33393-PC] }\end{array}$ & $\begin{array}{l}\text { Early, but not earliest, } \\
\text { Mississippian (middle } \\
\text { Kinderhookian through } \\
\text { Osagean). }\end{array}$ & $\begin{array}{l}\text { Synclydognathid } \\
\text { biofacies; normal- } \\
\text { marine, near restricted, } \\
\text { probably shallow- } \\
\text { water depositional } \\
\text { setting. }\end{array}$ & $\begin{array}{l}\text { Upper part of Kayak Shale(?); thicker limestone } \\
\text { overlying 97AK4. } \\
\text { Sample from medium-gray, fossiliferous limestone in } \\
\text { 3- to } 40 \text {-cm-thick beds with prominent cross- } \\
\text { laminae, ripples, and partings of sooty, black, } \\
\text { calcareous shale. Thin section is similar to 97AK4 } \\
\text { but lacks quartz silt. Skeletal wackestone/packstone; } \\
\text { bioclasts 30-40\%, chiefly <4 mm, include crinoid } \\
\text { ossicles, lesser brachiopods, bryozoans, calcareous } \\
\text { and siliceous spicules, and thin-shelled, articulated } \\
\text { ostracodes. Most bioclasts broken and abraded. } \\
\text { Local thin lenses rich in calcareous spicules. } \\
\text { Heavy-mineral concentrate is chiefly ferruginous } \\
\text { bioclasts and composite grains and lesser phosphatic } \\
\text { and phosphatized bioclasts (brachiopods, ostracodes, } \\
\text { and ichthyoliths). } \\
15 \text { kg of rock was processed. }\end{array}$ \\
\hline
\end{tabular}


Table 11. Conodont samples from the Endicott Group-Continued.

[All faunas identified by A.G. Harris. Structural units determined by L.E. Young. EMA, Endicott Mountains allochthon; PCA Picnic Creek allochthon. CAI, conodont color alteration index. Letters in field number refer to collector: AD, J.A. Dumoulin; AK, J.S. Kelley; Cx, S.M. Curtis; EK, I. Ellersieck; and MD, C.F. Mayfield. DDH, diamond drill hole. Interval thicknesses in drill holes are drilled thicknesses and have not been corrected for dip of bedding. All drill hole samples collected by J.A. Dumoulin and (or) A.G. Harris. Lithologic data under remarks are field descriptions unless otherwise indicated; thin section observations by J.A. Dumoulin. No, number; indet, indeterminate; loc., locality]

\begin{tabular}{|c|c|c|c|c|c|}
\hline $\begin{array}{l}\text { Locality No., } \\
\text { structural unit }\end{array}$ & $\begin{array}{l}\text { Quadrangle, } \\
\text { latitude n./ } \\
\text { longitude w. }\end{array}$ & $\begin{array}{c}\text { Conodont fauna and CAI } \\
\text { [field No.; USGS collection No.] }\end{array}$ & Age & $\begin{array}{c}\text { Conodont biofacies and } \\
\text { depositional } \\
\text { environment }\end{array}$ & Remarks \\
\hline $\begin{array}{l}90 \\
\text { EMA }\end{array}$ & $\begin{array}{l}\text { De Long Mtns. } \\
\text { A-2 } \\
68^{\circ} 02^{\prime} 38.2^{\prime \prime} / \\
162^{\circ} 36^{\prime} 9.5^{\prime \prime}\end{array}$ & $\begin{array}{l}\text { Barren } \\
{[97 \mathrm{AK} 115 \mathrm{~A}]}\end{array}$ & & & $\begin{array}{l}\text { Hunt Fork Shale. } \\
\text { Ripple-laminated, very fine to fine-grained sandstone, } \\
\text { locally calcareous with rare brachiopods, in } 0.5-\text { to } \\
1 \text {-m-thick beds. Thin section is very fine grained } \\
\text { sandstone, fairly well sorted, with a few crenulated } \\
\text { brachiopods; notable red-brown iron-rich material } \\
\text { between (and replaces?) grains. Clasts are } \sim 50 \% \\
\text { monocrystalline quartz, lesser chert, polycrystalline } \\
\text { quartz, mafic volcanic lithoclasts (including tuff } \\
\text { fragments?), and trace phosphate(?). } \\
7.0 \mathrm{~kg} \text { of rock was processed. }\end{array}$ \\
\hline $\begin{array}{l}103 \\
\text { EMA (Key } \\
\text { Creek plate) }\end{array}$ & $\begin{array}{l}\text { De Long Mtns. } \\
\text { B-1 } \\
68^{\circ} 21^{\prime} 21^{\prime \prime} / \\
162^{\circ} 02^{\prime} 16^{\prime \prime}\end{array}$ & $\begin{array}{l}\text { Barren } \\
{[00 \mathrm{AD} 17 \mathrm{~A}]}\end{array}$ & & & $\begin{array}{l}\text { Uppermost Kayak Formation stratigraphically below } \\
\text { contact with Kuna Formation; see table } 1 \text { for Kuna } \\
\text { samples from this locality. } \\
\text { Sample from limy concretions (10- to } 30 \text {-cm diameter) } \\
50 \text { to } 180 \mathrm{~cm} \text { below top of Kayak along August } \\
\text { Creek. Medium-gray- to grayish-orange-weathering, } \\
\text { medium-gray to medium-dark-gray, very fine } \\
\text { grained limestone. Thin-section is calcareous } \\
\text { spiculite. } \\
\text { Heavy-mineral concentrate includes pyritized spicules. } \\
10.8 \text { kg of rock was processed. } \\
\text { Loc. } 2 \text { (app. 1) of Dumoulin and others (2004). }\end{array}$ \\
\hline $\begin{array}{l}105 \\
\text { EMA (Key } \\
\text { Creek plate) }\end{array}$ & $\begin{array}{l}\text { De Long Mtns. } \\
\text { B-1 } \\
68^{\circ} 166^{\prime} 34^{\prime \prime} / \\
162^{\circ} 09^{\prime} 14.4^{\prime \prime}\end{array}$ & $\begin{array}{l}\text { Barren } \\
\text { [97AK55C] }\end{array}$ & & & $\begin{array}{l}\text { Upper part of Noatak Sandstone, below contact with } \\
\text { Kayak Shale. } \\
\text { Fine to very fine grained quartz sandstone with local } \\
\text { trough cross-beds, asymmetric ripples, burrows, and } \\
\text { sparse mud chips. Thin section is fine-grained } \\
\text { quartz>chert sandstone with silica cement. Clasts } \\
75 \% \text { monocrystalline quartz, with minor dolomite, } \\
\text { metamorphic and sedimentary lithoclasts, and } \\
\text { polycrystalline quartz. } \\
\text { Heavy-mineral concentrate includes phosphatic } \\
\text { bioclasts (brachiopod fragments) and rare } \\
\text { subrounded to well-rounded stable heavy minerals. } \\
5.9 \mathrm{~kg} \text { of rock was processed. }\end{array}$ \\
\hline
\end{tabular}


Table 11. Conodont samples from the Endicott Group-Continued.

[All faunas identified by A.G. Harris. Structural units determined by L.E. Young. EMA, Endicott Mountains allochthon; PCA Picnic Creek allochthon. CAI, conodont color alteration index. Letters in field number refer to collector: AD, J.A. Dumoulin; AK, J.S. Kelley; Cx, S.M. Curtis; EK, I. Ellersieck; and MD, C.F. Mayfield. DDH, diamond drill hole. Interval thicknesses in drill holes are drilled thicknesses and have not been corrected for dip of bedding. All drill hole samples collected by J.A.

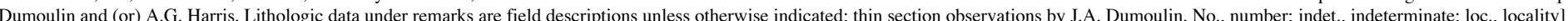

\begin{tabular}{|c|c|c|c|c|c|}
\hline $\begin{array}{l}\text { Locality No., } \\
\text { structural unit }\end{array}$ & $\begin{array}{l}\text { Quadrangle, } \\
\text { latitude n./ } \\
\text { longitude w. }\end{array}$ & $\begin{array}{c}\text { Conodont fauna and CAI } \\
\text { [field No.; USGS collection No.] }\end{array}$ & Age & $\begin{array}{c}\text { Conodont biofacies and } \\
\text { depositional } \\
\text { environment }\end{array}$ & Remarks \\
\hline $\begin{array}{l}115 \\
\text { EMA (Key } \\
\text { Creek plate) }\end{array}$ & $\begin{array}{l}\text { De Long Mtns. } \\
\text { A-1 } \\
68^{\circ} 08^{\prime} 10^{\prime \prime} / \\
162^{\circ} 30^{\prime} 48^{\prime \prime}\end{array}$ & $\begin{array}{l}5 \text { mid-Pa element fragments of the Bispathodus } \\
\text { aculeatus (Branson and Mehl) group } \\
\text { Unassigned elements: } \\
1 \mathrm{M}, 1 \mathrm{Sa} \text {, and } 1 \mathrm{Sc} \text { (fragments) } \\
14 \text { indet. bar, blade, and platform fragments } \\
\text { CAI=3 } \\
\text { [79EK274A] }\end{array}$ & \begin{tabular}{|l} 
latest Late Devonian-early \\
Early Mississippian (late \\
Famennian-Kinderhookian).
\end{tabular} & $\begin{array}{l}\text { Indeterminate (too few } \\
\text { conodonts); conodonts } \\
\text { that are present } \\
\text { indicate a high-energy, } \\
\text { shallow-water } \\
\text { depositional setting. }\end{array}$ & $\begin{array}{l}\text { Calcareous sandstone interval in Kayak Shale; } \\
\text { underlies Kuna Formation (Lisburne Group). } \\
\text { Fossil loc. 9, Curtis and others (1990). }\end{array}$ \\
\hline $\begin{array}{l}158 \\
\text { EMA (Key } \\
\text { Creek plate) }\end{array}$ & $\begin{array}{l}\text { De Long Mtns. } \\
\text { A-2 } \\
68^{\circ} 05^{\prime} 08.2^{\prime \prime} / \\
162^{\circ} 45^{\prime} 37.1^{\prime \prime}\end{array}$ & $\begin{array}{l}11 \text { Pa element fragments of a double-row } \\
\text { bispathodid } \\
1 \text { Pa element fragment of a single-row bispathodid } \\
\text { Kladognathus sp. indet. } \\
1 \mathrm{P}, 4 \mathrm{M}, 1 \mathrm{Sa} \text {, and } 2 \text { Sb-Sc element fragments } \\
1 \text { Pa element Polygnathus inornatus } \text { E.R. Branson } \\
1 \text { Pa element fragment Polygnathus } \text { sp. indet. } \\
1 \text { unassigned Sc element } \\
1 \text { phosphatized ammonoid steinkern } \\
\text { CAI=3 } \\
\text { [DDH } 587 \text { (E-NE of Red Dog Main deposit), } \\
\text { composite conodont sample: } 1764.7-66.7,1770.6- \\
74.7 \text {, and } 1775.0-76.0 \mathrm{ft} ; 33487-\mathrm{PC}]\end{array}$ & $\begin{array}{l}\text { early Early Mississippian } \\
\text { (Kinderhookian). }\end{array}$ & $\begin{array}{l}\text { Postmortem transport } \\
\text { within or from a } \\
\text { bispathodid- } \\
\text { kladognathid biofacies; } \\
\text { likely postmortem } \\
\text { transport from a } \\
\text { normal-marine } \\
\text { shallow-water } \\
\text { depositional setting. }\end{array}$ & $\begin{array}{l}\text { Kayak Shale, several hundred } \mathrm{m} \text { below contact with } \\
\text { Kuna Formation; Kayak in this drill hole may be } \\
\text { structurally thickened. See table } 3 \text { for Kuna sample } \\
\text { from this drill hole. } \\
\text { 1764.7-1776.0 ft: Irregular laminae and beds }(0.1-5 \mathrm{~cm} \\
\text { thick) of mudstone, siltstone, noncalcareous and } \\
\text { calcareous sandstone. Clastic strata have salt-and- } \\
\text { pepper color of medium-dark-gray to very light gray } \\
\text { (outer). Limy beds contain scattered small }(0.5 \mathrm{~cm} \\
\text { diameter) solitary corals and pelmatozoan debris. } \\
\text { Thin section data: } \\
1765.4,1770.2 \text { : Fine-grained quartz sandstone with } \\
\text { patchy carbonate cement and bioclasts. } \\
1772 \text { and } 1774.4 \text { : Crinoid grainstone with } \leq 25 \% \text { quartz } \\
\text { sand. Noncarbonate grains include chert, mica, and } \\
\text { metamorphic and sedimentary lithic clasts. } \\
\text { Heavy-mineral concentrate includes scarce } \\
\text { phosphatized bryozoan fragments and ichthyoliths. } \\
7.54 \text { kg of rock was processed. }\end{array}$ \\
\hline
\end{tabular}


Table 11. Conodont samples from the Endicott Group-Continued.

[All faunas identified by A.G. Harris. Structural units determined by L.E. Young. EMA, Endicott Mountains allochthon; PCA Picnic Creek allochthon. CAI, conodont color alteration index. Letters in field number refer to collector: AD, J.A. Dumoulin; AK, J.S. Kelley; Cx, S.M. Curtis; EK, I. Ellersieck; and MD, C.F. Mayfield. DDH, diamond drill hole. Interval thicknesses in drill holes are drilled thicknesses and have not been corrected for dip of bedding. All drill hole samples collected by J.A.
Dumoulin and (or) A.G. Harris. Lithologic data under remarks are field descriptions unless otherwise indicated; thin section observations by J.A. Dumoulin. No., number; indet, indeterminate; loc., locality]

\begin{tabular}{|c|c|c|c|c|c|}
\hline $\begin{array}{l}\text { Locality No., } \\
\text { structural unit }\end{array}$ & $\begin{array}{l}\text { Quadrangle, } \\
\text { latitude n./ } \\
\text { longitude w. }\end{array}$ & $\begin{array}{c}\text { Conodont fauna and CAI } \\
\text { [field No.; USGS collection No.] }\end{array}$ & Age & $\begin{array}{c}\text { Conodont biofacies and } \\
\text { depositional } \\
\text { environment }\end{array}$ & Remarks \\
\hline $\begin{array}{l}159 \\
\text { EMA (Key } \\
\text { Creek plate; } \\
\text { Port Road } \\
\text { succession of } \\
\text { Dumoulin and } \\
\text { others, 2004) }\end{array}$ & \begin{tabular}{|l|} 
Noatak D-3 \\
$67^{\circ} 51^{\prime} 04^{\prime \prime} /$ \\
$163^{\circ} 15^{\prime} 25^{\prime \prime}$
\end{tabular} & $\begin{array}{l}5 \text { Pa element fragments Bispathodus stablis } \\
\text { (Branson and Mehl) or Bi. utahensis Sandberg and } \\
\text { Gutschick } \\
1 \text { M element Idioprioniodus conjunctus } \\
\text { Kladognathus sp. indet. } \\
10 \mathrm{M} \text {, } 4 \mathrm{Sa} \text {, and } 17 \mathrm{Sb}-\mathrm{Sc} \text { elements, and } 4 \\
\text { fragments } \\
1 \text { unassigned M and } 1 \text { Sa elements } \\
46 \text { indet. bar, blade, and platform fragments } \\
\\
\text { CAI= 3 } \\
\text { [DDH 1103, composite conodont sample 4: 1376.2- } \\
\text { 76.4, 1376.6-76.7, 1377.2-77.6, 1379.5-79.8, } \\
\text { 1381.5-82.5, 1383.2-83.6, 1385.6-86; 1386.1- } \\
86.5,1404.4-06.5,1421-22.2 \mathrm{ft} ; 33661-\mathrm{PC}]\end{array}$ & $\begin{array}{l}\text { Early Mississippian (late } \\
\text { Kinderhookian-middle } \\
\text { Osagean); upper age } \\
\text { constraint based on } \\
\text { conodonts from overlying } \\
\text { Utukok Formation. }\end{array}$ & $\begin{array}{l}\text { Postmortem transport } \\
\text { within or from a } \\
\text { kladognathid biofacies. } \\
\text { This sample represents } \\
\text { a phosphatic lag } \\
\text { concentrate. }\end{array}$ & $\begin{array}{l}\text { Kayak Shale, below contact with Utukok Formation at } \\
\sim 1272 \mathrm{ft} \text {. See tables } 6 \text { and } 7 \text { for additional samples } \\
\text { from this drill hole. } \\
1376.2-1422.2 \mathrm{ft} \text { : Black to grayish-black to medium- } \\
\text { dark-gray noncalcareous shale and mudstone with } \\
\text { waxy to shiny luster, interbedded with very light } \\
\text { gray to medium-gray (outer), medium- to medium- } \\
\text { dark-gray (inner) bioclastic (crinoidal) limestone; } \\
\text { limestone in graded layers } 2 \mathrm{~cm} \text { to } 2 \mathrm{ft} \text { thick that are } \\
\text { locally bioturbated (shale not sampled for } \\
\text { conodonts). } \\
\text { Thin section data: } \\
1376 \mathrm{ft} \text { : Dark shale with laminae } 1-5 \text { mm thick of } \\
\text { calcareous and siliceous spicules. } \\
1376.2 \mathrm{ft} \text { : Dark shale with a partly silicified layer rich } \\
\text { in crinoid fragments with lesser spicules. } \\
1380 \mathrm{ft}: \text { Graded crinoid packstone with brachiopods, } \\
\text { noncarbonate mud matrix, and fitted fabric. } \\
1387 \mathrm{ft} \text { : Skeletal-intraclast grainstone with crinoids, } \\
\text { bryozoans, brachiopods, and phosphatic bioclasts. } \\
1403 \mathrm{ft} \text { Partly bioturbated bryozoan-crinoid } \\
\text { supportstone with seams of dark shale. } \\
\text { Heavy-mineral concentrate is chiefly variably pyritic, } \\
\text { partly phosphatic, very fine grained siliciclastic rock } \\
\text { fragments, phosphatic and phosphatized bioclasts } \\
\text { (chiefly ichthyoliths, minor brachiopods and } \\
\text { gastropod steinkerns), anhedral pyrite, and scarce } \\
\text { pyritized ostracode valves. } \\
7.9 \text { kg of rock was processed. }\end{array}$ \\
\hline $\begin{array}{l}175 \\
\text { EMA (Key } \\
\text { Creek plate) }\end{array}$ & $\begin{array}{l}\text { Noatak D-2 } \\
67^{\circ} 58^{\prime} 36^{\prime \prime} / \\
162^{\circ} 50^{\prime} 00^{\prime \prime}\end{array}$ & $\begin{array}{l}1 \text { gerontic Pa element Bispathodus stabilis } \\
\text { (Branson and Mehl) } \\
3 \text { Pa elements Patrognathus variabilis Rhodes, } \\
\text { Austin and Druce [pl. 3, figs. 7, 8] } \\
11 \text { Pa elements Polygnathus communis Branson } \\
\text { and Mehl [pl. 3, figs. 3, 4] } \\
1 \text { unassigned M element } \\
31 \text { indet. bar, blade, and platform fragments } \\
\text { CAI=4-4.5 } \\
\text { [81EK75F; 28584-PC] }\end{array}$ & $\begin{array}{l}\text { early Early Mississippian } \\
\text { (Kinderhookian). }\end{array}$ & $\begin{array}{l}\text { Mixed shelfal, likely } \\
\text { shallow-water } \\
\text { biofacies }\end{array}$ & $\begin{array}{l}\text { Sandstone member of Kayak Shale, above Kanayut } \\
\text { Conglomerate. } \\
\text { Limy, ferruginous encrinite interbedded with siltstone } \\
\text { and sandstone. } \\
\text { Heavy-mineral concentrate contains abundant } \\
\text { phosphatized steinkerns of a variety of ostracodes } \\
\text { and gastropods. }\end{array}$ \\
\hline
\end{tabular}


Table 11. Conodont samples from the Endicott Group-Continued.

[All faunas identified by A.G. Harris. Structural units determined by L.E. Young. EMA, Endicott Mountains allochthon; PCA Picnic Creek allochthon. CAI, conodont color alteration index. Letters in field number refer to collector: AD, J.A. Dumoulin; AK, J.S. Kelley; Cx, S.M. Curtis; EK, I. Ellersieck; and MD, C.F. Mayfield. DDH, diamond drill hole. Interval thicknesses in drill holes are drilled thicknesses and have not been corrected for dip of bedding. All drill hole samples collected by J.A. Dumoulin and (or) A.G. Harris. Lithologic data under remarks are field descriptions unless otherwise indicated; thin section observations by J.A. Dumoulin. No., number; indet, indeterminate; loc., locality]

\begin{tabular}{|c|c|c|c|c|c|}
\hline $\begin{array}{l}\text { Locality No., } \\
\text { structural unit }\end{array}$ & $\begin{array}{l}\text { Quadrangle, } \\
\text { latitude n./ } \\
\text { longitude w. }\end{array}$ & $\begin{array}{c}\text { Conodont fauna and CAI } \\
\text { [field No.; USGS collection No.] }\end{array}$ & Age & \begin{tabular}{|c|}
$\begin{array}{c}\text { Conodont biofacies and } \\
\text { depositional } \\
\text { environment }\end{array}$ \\
\end{tabular} & Remarks \\
\hline $\begin{array}{l}177 \\
\text { EMA (Key } \\
\text { Creek plate) }\end{array}$ & $\begin{array}{l}\text { Noatak C-4 } \\
67^{\circ} 37^{\prime} 05.1^{\prime \prime} / \\
163^{\circ} 42^{\prime} 17.2^{\prime \prime}\end{array}$ & $\begin{array}{l}1 \text { Bispathodus aculeatus aculeatus (Branson and } \\
\text { Mehl) } \\
6 \text { Pa elements Bispathodus stabilis (Branson and } \\
\text { Mehl) } \\
8 \text { Pa elements (juveniles and fragments) } \\
\text { Clydognathus? sp. or Patrognathus variabilis } \\
\text { Rhodes, Austin and Druce } \\
88 \text { Pa elements Polygnathus communis Branson } \\
\text { and Mehl } \\
3 \text { Pa elements Polygnathus inornatus } \text { E.R. Branson } \\
7 \text { Pa elements (mostly fragments) } \\
\text { Pseudopolygnathus orthoconstrictus (Thomas)? } \\
\text { Unassigned elements: } \\
7 \mathrm{M}, 2 \text { Sa, and } 1 \text { Sc } \\
5 \text { indet. bar, blade, and platform fragments } \\
\text { CAI=4.5 } \\
\text { [81EK108D; 28366-PC) }\end{array}$ & $\begin{array}{l}\text { early Early Mississippian } \\
\text { (Kinderhookian). }\end{array}$ & $\begin{array}{l}\text { Taxa are typical of very } \\
\text { shallow-water, often } \\
\text { quartz-sand-bearing } \\
\text { strata. }\end{array}$ & $\begin{array}{l}\text { Kayak Shale. } \\
\text { Sandy encrinite interbedded with marine sandstone and } \\
\text { siltstone. } \\
\text { Fauna is similar to that of Isikut member of the Kayak } \\
\text { Shale (Mull and others, 1997, Table 1, collns. } \\
\text { 92TM17A, 93TM35L, 93TM35C). }\end{array}$ \\
\hline $\begin{array}{l}178 \\
\text { EMA (Key } \\
\text { Creek plate) }\end{array}$ & $\begin{array}{l}\text { Noatak C-4 } \\
67^{\circ} 36^{\prime} 12^{\prime \prime} / \\
163^{\circ} 51^{\prime} 18^{\prime \prime}\end{array}$ & $\begin{array}{l}\text { Collection contains predominantly juveniles and } \\
\text { small fragments. } \\
1 \text { Pa element fragment Bispathodus stabilis } \\
\text { (Branson and Mehl) or Bi. utahensis Sandberg and } \\
\text { Gutschick } \\
\text { Hindeodus sp. indet. } \\
4 \text { Pa and } 1 \text { Sb elements } \\
1 \text { large Pa element Polygnathus inornatus E.R. } \\
\text { Branson } \\
3 \text { Pa elements Polygnathus sp. indet. (juveniles and } \\
\text { fragments) } \\
1 \text { juvenile Pa Pseudopolygnathus sp. indet. } \\
\text { Synclydognathus geminus (Hinde) } \\
1 \text { Pb and } 1 \text { Sa elements [pl. 3, figs. 5, 6] } \\
\text { Unassigned elements: } \\
4 \mathrm{M}(2 \text { morphotypes) and } 3 \text { Sc (3 morphotypes) } \\
43 \text { small indet. bar, blade, and platform fragments } \\
\text { CAI=4.5-5 } \\
\text { [81MD41E; } 28367-P C]\end{array}$ & $\begin{array}{l}\text { early Early Mississippian } \\
\text { (middle-late } \\
\text { Kinderhookian). }\end{array}$ & $\begin{array}{l}\text { Size and condition of } \\
\text { conodonts indicates a } \\
\text { winnow from a } \\
\text { shallow-water, normal- } \\
\text { marine environment. }\end{array}$ & $\begin{array}{l}\text { Kayak Shale. } \\
\text { Rubbly subcrop of gray limestone interbedded with } \\
\text { siltstone. }\end{array}$ \\
\hline
\end{tabular}


Table 12. Conodont samples from Devonian carbonate rocks

[All faunas identified by A.G. Harris. Structural units determined by L.E. Young. KRA, Kelly River allochthon; PCA, Picnic Creek allochthon. CAI, conodont color alteration index. Letters in field number refer to collector: AD, J.A. Dumoulin; AK, J.S. Kelley; Cx, S.M. Curtis; MD, C.F. Mayfield; and RB, R.B. Blodgett. Lithologic data under remarks are field descriptions unless otherwise indicated; thin section observations by J.A. Dumoulin. No., number; indet., indeterminate; loc., locality]

\begin{tabular}{|c|c|c|c|c|c|}
\hline $\begin{array}{l}\text { Locality No., } \\
\text { Structural unit }\end{array}$ & $\begin{array}{l}\text { Quadrangle, } \\
\text { latitude n./ } \\
\text { longitude w. }\end{array}$ & $\begin{array}{c}\text { Conodont fauna and CAI } \\
\text { [field No.; USGS collection No.] }\end{array}$ & Age & $\begin{array}{l}\text { Conodont biofacies and } \\
\text { depositional environment }\end{array}$ & Remarks \\
\hline $\begin{array}{ll}11 \\
\text { PCA }\end{array}$ & $\begin{array}{l}\text { De Long Mtns. } \\
\text { A-3 } \\
68^{\circ} 10^{\prime} 12^{\prime \prime} / \\
163^{\circ} 28^{\prime} 42^{\prime \prime}\end{array}$ & $\begin{array}{l}\text { 1 Dvorakia? sp. indet. } \\
12 \text { I elements Icriodus symmetricus Branson and } \\
\text { Mehl? } \\
16 \text { I element fragments Icriodus sp. indet. } \\
2 \text { Pa elements Polygnathus cristatus Hinde } \\
1 \text { Pa element fragment Polygnathus cf. Po. } \\
\text { latifossatus Wirth } \\
9 \text { Pa element fragments Polygnathus linguiformis } \\
\text { linguiformis Hinde } \\
\text { Unassigned elements: } \\
1 \mathrm{M} \text { and } 2 \mathrm{Sc} \\
70 \text { indet. bar, blade, and platform fragments } \\
\text { CAI=3.5 } \\
\text { [8-16-83M; } 10860-\mathrm{SD}]\end{array}$ & $\begin{array}{l}\text { late Middle Devonian } \\
\text { (early-late Givetian; } \\
\text { upper Po. varcus } \\
\text { Subzone through at least } \\
\text { K. disparilis Zone). }\end{array}$ & $\begin{array}{l}\text { Icriodid biofacies; high- } \\
\text { energy reef to near reef } \\
\text { and shoal depositional } \\
\text { setting. }\end{array}$ & $\begin{array}{l}\text { Medium-light-gray, medium-gray- and grayish-orange- } \\
\text { weathering, massive-bedded, crinoidal, bioclastic } \\
\text { grainstone. Thin section is partly dolomitized, } \\
\text { loosely packed crinoid grainstone with rare bryozoan } \\
\text { and brachiopod fragments. Crinoid ossicles (to } 5 \mathrm{~mm} \\
\text { in diameter) make up } 80-90 \% \text { of bioclasts. } \\
5.9 \text { kg of rock was processed. } \\
\text { Sample collected by A.G. Harris. }\end{array}$ \\
\hline $\begin{array}{l}12 \\
\text { PCA (Amaruk } \\
\text { plate) }\end{array}$ & $\begin{array}{l}\text { De Long Mtns. } \\
\text { A-3 } \\
68^{\circ} 10^{\prime} 14.6^{\prime \prime} / \\
163^{\circ} 26^{\prime} 13.4^{\prime \prime}\end{array}$ & $\begin{array}{l}\text { 4 Pa element fragments Polygnathus spp. indet. of } \\
\text { Devonian morphotype } \\
11 \text { indet. bar, blade, and platform fragments } \\
\text { CAI }=3-4 \\
\text { [79MD116B] }\end{array}$ & $\begin{array}{l}\text { Middle and (or) Late } \\
\text { Devonian (Eifelian- } \\
\text { middle Frasnian and } \\
\text { (or) Famennian, but not } \\
\text { late Frasnian). }\end{array}$ & $\begin{array}{l}\text { Indeterminate (too few } \\
\text { conodonts); taxa present } \\
\text { and their condition } \\
\text { indicate postmortem } \\
\text { transport within or from a } \\
\text { shelf, probably a high- } \\
\text { energy environment. } \\
\end{array}$ & Fossil loc. 28, Mayfield and others (1990). \\
\hline $\begin{array}{l}14 \\
\text { PCA }\end{array}$ & $\begin{array}{l}\text { De Long Mtns. } \\
\text { A-3 } \\
68^{\circ} 08^{\prime} 12^{\prime \prime} / \\
163^{\circ} 41^{\prime} 06^{\prime \prime}\end{array}$ & $\begin{array}{l}5 \text { indet. bar, blade, and platform fragments of post- } \\
\text { Early Ordovician morphotype } \\
\text { CAI }=3.5 \\
{[8-16-83 \mathrm{~L}]}\end{array}$ & $\begin{array}{l}\text { Middle Ordovician- } \\
\text { Permian }\end{array}$ & $\begin{array}{l}\text { Indeterminate (too few } \\
\text { conodonts). }\end{array}$ & $\begin{array}{l}\text { Outcrop consists of } 85 \% \text { calcareous shale and } 15 \% \\
\text { slightly argillaceous, very fine grained, rippled, } \\
\text { medium-gray, light-gray- to yellowish-gray- } \\
\text { weathering limestone with flute casts. Thin section is } \\
\text { pelloidal, ferroan calcisiltite with seams rich in } \\
\text { ferroan dolomite, quartz, feldspar, and white mica. } \\
5.78 \text { kg of rock was processed. } \\
\text { Sample collected by A.G. Harris. }\end{array}$ \\
\hline
\end{tabular}


Table 12. Conodont samples from Devonian carbonate rocks - Continued.

[All faunas identified by A.G. Harris. Structural units determined by L.E. Young. KRA, Kelly River allochthon; PCA, Picnic Creek allochthon. Letters in field number refer to collector: AD, J.A. Dumoulin; AK, J.S. Kelley: Cx, S.M. Curtis: MD, C.F. Mayfield; and RB, R.B. Blodgett. CAI, conodont color alteration index. Lithologic data under remarks are field descriptions unless otherwise indicated; thin section observations by J.A. Dumoulin. No., number; indet, indeterminate; loc, localityl

\begin{tabular}{|c|c|c|c|c|c|}
\hline $\begin{array}{l}\text { Locality No., } \\
\text { Structural unit }\end{array}$ & $\begin{array}{l}\text { Quadrangle, } \\
\text { latitude n./ } \\
\text { longitude w. }\end{array}$ & $\begin{array}{c}\text { Conodont fauna and CAI } \\
\text { [field No.; USGS collection No.] }\end{array}$ & Age & $\begin{array}{l}\text { Conodont biofacies and } \\
\text { depositional environment }\end{array}$ & Remarks \\
\hline $\begin{array}{ll}26 \\
\text { KRA }\end{array}$ & \begin{tabular}{|l} 
De Long Mtns. \\
A-3 \\
$68^{\circ} 05^{\prime} 42^{\prime \prime} /$ \\
$163^{\circ} 27^{\prime} 42^{\prime \prime}$
\end{tabular} & $\begin{array}{l}\text { Polygnathus cooperi cooperi Klapper } \\
81 \mathrm{~Pa}, 5 \mathrm{~Pb}, 10 \mathrm{M}, 4 \mathrm{Sa}, 3 \mathrm{Sb} \text {, and } 4 \mathrm{Sc} \text { elements } \\
247 \text { indet. bar, blade, and platform fragments } \\
\text { CAI }=3.5 \\
\text { [8-16-83I; 10859-SD] }\end{array}$ & $\begin{array}{l}\text { very latest Early-early } \\
\text { Middle Devonian (latest } \\
\text { Emsian-early Eifelian; } \\
\text { Po. serotinus Zone-Po. } \\
\text { costatus costatus Zone). }\end{array}$ & $\begin{array}{l}\text { Polygnathid biofacies; shelf } \\
\text { or platform depositional } \\
\text { setting. The preservation } \\
\text { of this collection indicates } \\
\text { a relatively high-energy } \\
\text { environment and } \\
\text { therefore shallow water. } \\
\end{array}$ & $\begin{array}{l}\text { Fossiliferous, crinoidal, medium-light-gray, light-gray- } \\
\text { weathering packstone. From talus block on slope of } \\
\text { mountain at } 1250 \mathrm{ft} \text {. Thin section is coarse-grained } \\
\text { pelmatozoan packstone with lesser brachiopods; } \\
\text { largely dolomitized matrix. } \\
7.4 \text { kg of rock was processed. } \\
\text { Sample collected by A.G. Harris. }\end{array}$ \\
\hline $\begin{array}{ll}27 \\
\text { KRA }\end{array}$ & $\begin{array}{l}\text { De Long Mtns. } \\
\text { A-3 } \\
68^{\circ} 05^{\prime} 32^{\prime \prime} / \\
163^{\circ} 27^{\prime} 54^{\prime \prime}\end{array}$ & $\begin{array}{l}\text { Polygnathus linguiformis Hinde } \\
8 \mathrm{~Pa}, 1 \mathrm{M} \text {, and } 1 \mathrm{Sb} \text { (mostly fragments) } \\
77 \text { indet. bar, blade, and platform fragments } \\
\mathrm{CAI}=3-3.5 \\
{[8-16-83 \mathrm{H} ; 10858-\mathrm{SD}]}\end{array}$ & $\begin{array}{l}\text { very latest Early-early } \\
\text { early Late Devonian } \\
\text { (latest Emsian-early } \\
\text { Frasnian; Po. serotinus } \\
\text { Zone-Lower Pa. hassi } \\
\text { Zone). }\end{array}$ & $\begin{array}{l}\text { Indeterminate (too few } \\
\text { conodonts); postmortem } \\
\text { transport within or from } \\
\text { the polygnathid biofacies, } \\
\text { probably normal-marine, } \\
\text { shallow-water } \\
\text { depositional environment. }\end{array}$ & $\begin{array}{l}\text { Medium-gray, fine-grained, fossiliferous, rubbly and } \\
\text { massive-bedded limestone. Thin section is packed, } \\
\text { skeletal-pelloidal packstone. Bioclasts include } \\
\text { pelmatozoan, bryozoan, and brachiopod fragments; } \\
\text { many clasts abraded and micritized. } \\
5.1 \text { kg of rock was processed. } \\
\text { Sample collected by A.G. Harris. }\end{array}$ \\
\hline $\begin{array}{ll}28 \\
\text { KRA }\end{array}$ & $\begin{array}{l}\text { De Long Mtns. } \\
\text { A-3 } \\
68^{\circ} 05^{\prime} 03^{\prime \prime} / \\
163^{\circ} 28^{\prime} 53.5^{\prime \prime}\end{array}$ & $\begin{array}{l}1 \text { posterior fragment Pa element Polygnathus } \\
\text { linguiformis Hinde } \\
1 \text { Pa element Polygnathus pseudofoliatus } \\
\text { Wittekind } \\
1 \text { indet. bar fragment } \\
\text { CAI }=3 \\
\text { [84RB118] }\end{array}$ & $\begin{array}{l}\text { late Middle Devonian } \\
\text { (lower half of Givetian; } \\
\text { Lower-Middle } P \text {. varcus } \\
\text { Subzones), based on } \\
\text { overlapping ages of } \\
\text { brachiopods and } \\
\text { conodonts. Polygnathus } \\
\text { pseudofoliatus ranges } \\
\text { from the } T \text {. } \text { k. australis } \\
\text { Zone into the Middle } P \text {. } \\
\text { varcus Subzone. }\end{array}$ & $\begin{array}{l}\text { Likely a polygnathid } \\
\text { biofacies; probable } \\
\text { normal-marine } \\
\text { depositional environment. }\end{array}$ & $\begin{array}{l}\text { Dark-gray lime mudstone with common silicified } \\
\text { fossils, including brachiopods (predominantly } \\
\text { stringocephalids), stromatoporoids, solitary rugose } \\
\text { corals, and gastropods. The brachiopods indicate a } \\
\text { Givetian age (Blodgett and Dutro, 1992). }\end{array}$ \\
\hline $\begin{array}{l}29 \\
\text { KRA (Wulik } \\
\text { Peaks plate) }\end{array}$ & $\begin{array}{l}\text { De Long Mtns. } \\
\text { A-3 } \\
68^{\circ} 04^{\prime} 59^{\prime \prime} / \\
163^{\circ} 21^{\prime} 59.6^{\prime \prime}\end{array}$ & $\begin{array}{l}\text { Barren } \\
{[99 \mathrm{AD} 22 \mathrm{~A}]}\end{array}$ & & & $\begin{array}{l}\text { Massive, castle-forming, light-gray-weathering, dark- } \\
\text { grayish-brown micritic limestone with rare small } \\
\text { bioclasts and clasts(?), slightly fetid, in } 30 \text { - to } 50- \\
\text { cm-thick beds. Thin section is peloidal-skeletal } \\
\text { supportstone. } \\
\text { This unit intruded by mafic dike(?) (map unit JPm4 of } \\
\text { Mayfield and others, 1990) } \sim 0.6 \mathrm{mi} \text { southwest of this } \\
\text { locality. } \\
9.35 \mathrm{~kg} \text { of rock was processed. }\end{array}$ \\
\hline
\end{tabular}


Table 12. Conodont samples from Devonian carbonate rocks - Continued.

[All faunas identified by A.G. Harris. Structural units determined by L.E. Young. KRA, Kelly River allochthon; PCA, Picnic Creek allochthon. Letters in field number refer to collector: AD, J.A. Dumoulin; AK, J.S. Kelley: Cx, S.M. Curtis: MD, C.F. Mayfield; and RB, R.B. Blodgett. CAI, conodont color alteration index. Lithologic data under remarks are field descriptions unless otherwise indicated; thin section observations by J.A. Dumoulin. No., number; indet, indeterminate; loc, localityl

\begin{tabular}{|c|c|c|c|c|c|}
\hline $\begin{array}{l}\text { Locality No., } \\
\text { Structural unit }\end{array}$ & $\begin{array}{l}\text { Quadrangle, } \\
\text { latitude n./ } \\
\text { longitude w. }\end{array}$ & $\begin{array}{c}\text { Conodont fauna and CAI } \\
\text { [field No.; USGS collection No.] }\end{array}$ & Age & $\begin{array}{l}\text { Conodont biofacies and } \\
\text { depositional environment }\end{array}$ & Remarks \\
\hline $\begin{array}{l}97 \\
\text { KRA } \\
\text { (Amphitheatre } \\
\text { plate) }\end{array}$ & $\begin{array}{l}\text { De Long Mtns. } \\
\text { B-1 } \\
68^{\circ} 20^{\prime} 19.2^{\prime \prime} / \\
162^{\circ} 18^{\prime} 15.2^{\prime \prime}\end{array}$ & $\begin{array}{l}2 \text { posterior Pa element fragments Polygnathus sp. } \\
\text { indet. of Middle Devonian-late Late } \\
\text { Mississippian morphotype } \\
1 \text { unassigned Sc element } \\
1 \text { indet. bar fragment } \\
\text { CAI=3 } \\
\text { [79MD47] }\end{array}$ & $\begin{array}{l}\text { Middle Devonian-late } \\
\text { Late Mississippian. }\end{array}$ & $\begin{array}{l}\text { Indeterminate (too few } \\
\text { conodonts). }\end{array}$ & Fossil loc. 10, Curtis and others (1990). \\
\hline $\begin{array}{l}98 \\
\text { KRA } \\
\text { (Amphitheatre } \\
\text { plate) }\end{array}$ & \begin{tabular}{|l|} 
De Long Mtns. \\
B-1 \\
$68^{\circ} 19^{\prime} 47.7^{\prime \prime} /$ \\
$162^{\circ} 19^{\prime} 19.4^{\prime \prime}$ \\
\end{tabular} & $\begin{array}{l}2 \text { indet. bar and blade fragments } \\
\text { CAI }=3 \\
{[00 \mathrm{AD} 13 \mathrm{~A}]}\end{array}$ & Devonian-Mississippian & $\begin{array}{l}\text { Indeterminate (too few } \\
\text { conodonts). }\end{array}$ & $\begin{array}{l}\text { Top of Devonian(?), overlain at this locality by the } \\
\text { micritic limestone unit of the Lisburne Group (see } \\
\text { table } 8 \text { for sample from this unit). } \\
\text { Crudely massively bedded, medium-gray-weathering, } \\
\text { dark-gray lime mudstone with dark-yellowish-gray } \\
\text { mottles(?) or zones. Thin section is peloidal } \\
\text { supportstone with rare calcispheres. } \\
9.4 \text { kg of rock was processed. }\end{array}$ \\
\hline $\begin{array}{l}101 \\
\text { KRA (Kelly } \\
\text { plate) }\end{array}$ & \begin{tabular}{|l|} 
De Long Mtns. \\
B-1 \\
$68^{\circ} 21^{\prime} 3.7^{\prime \prime} /$ \\
$162^{\circ} 12^{\prime} 12.1^{\prime \prime}$ \\
\end{tabular} & $\begin{array}{l}5 \text { Pa elements Polygnathus angusticostatus } \\
\text { Wittekindt } \\
\text { Vicarious elements of Polygnathus spp.: } \\
2 \mathrm{M}, 1 \mathrm{Sb} \text {, and } 5 \mathrm{Sc} \text { ( } 2 \text { morphotypes) elements } \\
2 \mathrm{~Pa} \text { element fragments Polygnathus sp. indet. } \\
20 \text { indet. bar, blade, and platform fragments } \\
\text { CAI=3-3.5 } \\
\text { [99AD17A; 12673-SD] }\end{array}$ & $\begin{array}{l}\text { middle Middle Devonian } \\
\text { (middle Eifelian into } \\
\text { earliest Givetian). Po. } \\
\text { angusticostatus is } \\
\text { known to range from the } \\
\text { T. } \text { k. australis Zone } \\
\text { through(?) the Po. } x \text {. } \\
\text { ensensis Zone. }\end{array}$ & $\begin{array}{l}\text { Indeterminate (too few } \\
\text { conodonts), but likely a } \\
\text { relatively shallow water } \\
\text { shelf or platform } \\
\text { depositional setting. }\end{array}$ & $\begin{array}{l}\text { Sample taken near base of 6-m-thick outcrop. Late } \\
\text { Middle Devonian brachiopods (fossil loc. } 38 \text { of } \\
\text { Curtis et al., 1990) near here. } \\
\text { Grayish-black, medium-gray-weathering, fetid, } \\
\text { dolomitic lime mudstone in uneven 5- to 7-cm-thick } \\
\text { nodular beds. Thin section is a fine-grained, euhedral } \\
\text { dolomite mosaic. } \\
10.9 \text { kg of rock was processed. }\end{array}$ \\
\hline $\begin{array}{l}104 \\
\text { KRA (Kelly } \\
\text { plate) }\end{array}$ & $\begin{array}{l}\text { De Long Mtns. } \\
\text { B-1 } \\
68^{\circ} 18^{\prime} 52.6^{\prime \prime} / \\
162^{\circ} 08^{\prime} 59.3^{\prime \prime}\end{array}$ & $\begin{array}{l}9 \text { Pa incomplete elements Apatognathus varians } \\
\text { Branson and Mehl } \\
1 \text { Pa element Polygnathus communis communis } \\
\text { Branson and Mehl } \\
5 \text { Pa elements Polygnathus semicostatus Branson } \\
\text { and Mehl } \\
\text { Unassigned elements: } \\
1 \text { Pa (fragment), } 2 \mathrm{~Pb} \text {, and } 1 \mathrm{Sc} \\
50 \text { indet. bar, blade, and platform fragments } \\
\text { CAI=3 } \\
\text { [79CX60A; 10013-SD] }\end{array}$ & $\begin{array}{l}\text { late Late Devonian } \\
\text { (Famennian, not earliest } \\
\text { or latest; within Middle } \\
\text { Pa. crepida Zone to } \\
\text { within Lower Si. } \\
\text { praesulcata expansa } \\
\text { Zone). }\end{array}$ & $\begin{array}{l}\text { Indeterminate (too few } \\
\text { conodonts); species that } \\
\text { are present indicate a } \\
\text { shallow- to middle-shelf } \\
\text { depositional setting. }\end{array}$ & $\begin{array}{l}2.3 \mathrm{~kg} \text { of rock was processed. } \\
\text { Fossil loc. } 12 \text { of Curtis and others (1990). }\end{array}$ \\
\hline
\end{tabular}


Table 12. Conodont samples from Devonian carbonate rocks - Continued.

[All faunas identified by A.G. Harris. Structural units determined by L.E. Young. KRA, Kelly River allochthon; PCA, Picnic Creek allochthon. Letters in field number refer to collector: AD, J.A. Dumoulin; AK, J.S. Kelley: Cx, S.M. Curtis: MD, C.F. Mayfield; and RB, R.B. Blodgett. CAI, conodont color alteration index. Lithologic data under remarks are field descriptions unless otherwise indicated; thin section observations by J.A. Dumoulin. No., number; indet, indeterminate; loc., locality]

\begin{tabular}{|c|c|c|c|c|c|}
\hline $\begin{array}{l}\text { Locality No., } \\
\text { Structural unit }\end{array}$ & $\begin{array}{l}\text { Quadrangle, } \\
\text { latitude n./ } \\
\text { longitude w. }\end{array}$ & $\begin{array}{c}\text { Conodont fauna and CAI } \\
\text { [field No.; USGS collection No.] }\end{array}$ & Age & $\begin{array}{l}\text { Conodont biofacies and } \\
\text { depositional environment }\end{array}$ & Remarks \\
\hline \multirow[t]{3}{*}{$\begin{array}{l}108 \\
\text { KRA }\end{array}$} & \multirow[t]{3}{*}{$\begin{array}{l}\text { De Long Mtns. } \\
\text { A-1 } \\
68^{\circ} 14 . ' 54^{\prime \prime} / \\
162^{\circ} 14^{\prime} 30^{\prime \prime}\end{array}$} & $\begin{array}{l}\text { Barren } \\
{[97 \mathrm{AK} 61 \mathrm{~A}]}\end{array}$ & & & $\begin{array}{l}\text { Sample taken near base of } \sim 500 \text {-ft-thick measured } \\
\text { section of Devonian limestone overlain by Utukok } \\
\text { Formation; see table } 7 \text { for Utukok samples from this } \\
\text { locality. } \\
\text { Light-medium-gray-weathering, medium-gray } \\
\text { limestone with local gastropods and shell hash. Thin } \\
\text { section is micrite with }<10 \% \text { bioclasts, including } \\
\text { calcispheres, lesser ostracodes, and calcareous } \\
\text { spicules(?). Local lenses of peloidal supportstone; } \\
\text { peloids } 20-60 \mu \mathrm{m} \text {, rarely to } 300 \mu \mathrm{m} \text {. Abundant } \\
\text { calcite veins, and some stylolites with trace quartz } \\
\text { silt. } \\
7.2 \mathrm{~kg} \text { of rock was processed. }\end{array}$ \\
\hline & & $\begin{array}{l}1 \text { indet. conodont fragment } \\
\text { CAI }=\sim 3 \\
\text { [97AK62] }\end{array}$ & Ordovician-Permian & Indeterminate. & $\begin{array}{l}\text { Sample taken } \sim 160 \mathrm{ft} \text { above } 97 \mathrm{AK} 61 \mathrm{~A} . \\
\text { Light-medium-gray-weathering, medium-light-gray to } \\
\text { medium-gray limestone with possible peloids, } \\
\text { bioclasts, and symmetrical ripples. Thin section is } \\
\text { peloidal supportstone grading to micrite. Trace } \\
\text { quartz silt along stylolites and abundant calcite } \\
\text { veins. Less than } 5 \% \text { bioclasts, mostly calcispheres. } \\
\text { Possible fenestrae to } 500 \mu \mathrm{m} \text { filled with calcite spar. } \\
\text { Trace dolomite rhombs replaced by silica. } \\
5.6 \mathrm{~kg} \text { of rock was processed. }\end{array}$ \\
\hline & & $\begin{array}{l}1 \text { indet. bar or blade fragment } \\
1 \text { ichthyolith } \\
\text { CAI=3 } \\
\text { [97AK63] }\end{array}$ & Devonian-Permian & Indeterminate. & $\begin{array}{l}\text { Sample taken } \sim 100 \mathrm{ft} \text { above } 97 \mathrm{AK} 62 . \text { Limestone } \\
\text { similar to that at } 97 \mathrm{AK} 61 \text { and } 62 \text {. Thin section is } \\
\text { skeletal-peloidal-mud lump packstone with lenses of } \\
\text { grainstone. Bioclasts include calcispheres, } \\
\text { brachiopods(?), and abundant algae(?). Some } \\
\text { algae(?) have bored micritic rims. } \\
7.3 \mathrm{~kg} \text { of rock was processed. }\end{array}$ \\
\hline $\begin{array}{l}109 \\
\text { KRA (Kelly } \\
\text { plate) }\end{array}$ & $\begin{array}{l}\text { De Long Mtns. } \\
\text { A-1 } \\
68^{\circ} 14^{\prime} 31.6^{\prime \prime} / \\
162^{\circ} 05^{\prime} 31.2^{\prime \prime}\end{array}$ & $\begin{array}{l}\text { No conodonts or other phosphatic or phosphatized } \\
\text { fossils were found. } \\
\text { [03AD23A] }\end{array}$ & & & $\begin{array}{l}\text { Medium- to medium-light-gray dolostone, weathers } \\
\text { light gray, with very fetid (petroliferous) odor. } \\
\text { Outcrop } \sim 0.5 \mathrm{~m} \text { thick; cut by numerous calcite and } \\
\text { quartz veins. Many fractures contain abundant, large } \\
\text { patches of dead oil. Beds } \sim 10 \mathrm{~cm} \text {, uneven. Locally } \\
\text { abundant corals and amphiporid stromatoporoids. } \\
\text { Thin section is dolomite mosaic with ghostly } \\
\text { bioclasts, probably mostly Amphipora sp. } \\
6.4 \mathrm{~kg} \text { of rock was processed. }\end{array}$ \\
\hline
\end{tabular}


Table 13. Conodont samples from the Noatak quadrangle

[All faunas identified by A.G. Harris. ${ }^{+}$Structural unit determined by L.E. Young. All other structural and stratigraphic units as reported by C.F. Mayfield and co-workers. EMA, Endicott Mountains allochthon; KRA, Kelly River allochthon; NRA, Nuka Ridge allochthon; PCA, Picnic Creek allochthon. CAI, conodont color alteration index. Letters in field number refer to collector: EK, I. Ellersieck; MD, C.F. Mayfield; and TR, I.L. Tailleur. *, age revised by A.G. Harris in 2000. Lithologic data under remarks are field descriptions unless otherwise indicated; thin section observations by J.A. Dumoulin. No., number; loc., locality]

\begin{tabular}{|c|c|c|c|c|}
\hline $\begin{array}{l}\text { Locality No., } \\
\text { structural unit }\end{array}$ & $\begin{array}{l}\text { Quadrangle, } \\
\text { latitude } \mathrm{n} . / \\
\text { longitude } \mathrm{w} .\end{array}$ & $\begin{array}{l}\text { Age of conodont fauna and CAI } \\
\text { [field No.; USGS collection No.] }\end{array}$ & Stratigraphic unit & Remarks \\
\hline $\begin{array}{l}161 \\
\text { KRA (Kelly } \\
\text { plate) }\end{array}$ & \begin{tabular}{|l|} 
Noatak D-6 \\
$67^{\circ} 57^{\prime} 34.5^{\prime \prime} /$ \\
$164^{\circ} 40^{\prime} 30^{\prime \prime}$ \\
\end{tabular} & $\begin{array}{l}\text { Late Mississippian-early Early Permian* } \\
\text { CAI }=1-1.5 \\
{[81 \mathrm{MD} 29 \mathrm{C} ; 28585-\mathrm{PC}]} \\
\end{array}$ & Kogruk Formation & $\begin{array}{l}\text { Limestone with gray chert. } \\
\text { Fossil loc. } 5 \text { in Mayfield and others (1987). }\end{array}$ \\
\hline $\begin{array}{l}162 \\
\text { KRA (Kelly } \\
\text { plate) }\end{array}$ & \begin{tabular}{|l|} 
Noatak D-6 \\
$67^{\circ} 57^{\prime} 00^{\prime \prime} /$ \\
$164^{\circ} 39^{\prime} 06^{\prime \prime}$ \\
\end{tabular} & $\begin{array}{l}\text { Late Mississippian (late Meramecian-late Chesterian) } \\
\text { CAI=1 } \\
\text { [81MD29H; 28586-PC] }\end{array}$ & Kogruk Formation & $\begin{array}{l}\text { Limestone with silicified zones containing crinoidal debris. } \\
\text { Fossil loc. } 4 \text { in Mayfield and others (1987). }\end{array}$ \\
\hline \multirow[t]{3}{*}{$\begin{array}{l}163 \\
\text { NRA (Bogie } \\
\text { plate) }\end{array}$} & \multirow[t]{3}{*}{$\begin{array}{l}\text { Noatak D-6 } \\
67^{\circ} 57^{\prime} 18^{\prime \prime} / \\
164^{\circ} 33^{\prime} 36^{\prime \prime}\end{array}$} & \begin{tabular}{|l|} 
Devonian-Permian \\
CAI=1 \\
[81EK141] \\
\end{tabular} & \multirow[t]{3}{*}{ Nuka Formation } & $\begin{array}{l}\text { Sandy limestone. } \\
\text { Fossil loc. } 2 \text { in Mayfield and others (1987). }\end{array}$ \\
\hline & & $\begin{array}{l}\text { Late Mississippian-middle Middle Pennsylvanian (late } \\
\text { Meramecian-early Atokan) } \\
\text { CAI=1 } \\
\text { [8-16-83J; 29233-PC] }\end{array}$ & & $\begin{array}{l}\text { Very light gray to pinkish-gray, terrigenous, fossiliferous grainstone } \\
\text { that contains plagioclase feldspar and quartz granules and sand. } \\
\text { Thin section is bioclastic grainstone with } \leq 7 \% \text { rounded to } \\
\text { subangular noncarbonate detritus, mainly monocrystalline quartz } \\
\text { and lesser plagioclase and microcline feldspar. Bioclasts chiefly } \\
\text { crinoid ossicles and subordinate bryozoan and brachiopod } \\
\text { fragments. Trace amounts of phosphate and glauconite. } \\
6.5 \text { kg of rock was processed. } \\
\text { Sample collected by A.G. Harris. }\end{array}$ \\
\hline & & $\begin{array}{l}\text { Late Mississippian-middle Middle Pennsylvanian (late } \\
\text { Meramecian-early Atokan) } \\
\text { CAI=1 } \\
\text { [8-16-83K; 29234-PC] }\end{array}$ & & $\begin{array}{l}\text { Grayish-red, terrigenous, arkosic wackestone with echinoderm } \\
\text { debris, plagioclase feldspar laths, and subrounded sand, granules, } \\
\text { and pebbles of quartz. Thin section is very poorly sorted, coarse- } \\
\text { grained bioclastic grainstone with } 10 \text { to } 15 \% \text { chiefly rounded } \\
\text { noncarbonate detritus (monocrystalline and polycrystalline quartz, } \\
\text { plagioclase and microcline feldspar, and glauconite). Bioclasts } \\
\text { mainly crinoid and diverse bryozoan fragments. Minor } \\
\text { phosphate(?) and non-ferroan dolomite. } \\
5.78 \text { kg of rock was processed. } \\
\text { Sample collected by A.G. Harris. } \\
\end{array}$ \\
\hline $\begin{array}{l}164 \\
\text { PCA }\end{array}$ & \begin{tabular}{|l|} 
Noatak D-6 \\
$67^{\circ} 53^{\prime} 36^{\prime \prime} /$ \\
$164^{\circ} 55^{\prime} 48^{\prime \prime}$ \\
\end{tabular} & \begin{tabular}{|l|} 
Early Mississippian-Permian \\
CAI $=3$ \\
[81MD9E $]$
\end{tabular} & Kayak Shale & $\begin{array}{l}\text { Buff-weathering limestone between gray shale and black chert. } \\
\text { Fossil loc. } 6 \text { in Mayfield and others (1987). }\end{array}$ \\
\hline $\begin{array}{l}165 \\
\text { NRA (Bogie } \\
\text { plate) }\end{array}$ & \begin{tabular}{|l|} 
Noatak D-6 \\
$67^{\circ} 52^{\prime} 12 " /$ \\
$164^{\circ} 45^{\prime} 30^{\prime \prime}$
\end{tabular} & $\begin{array}{l}\text { Late Mississippian-middle Middle Pennsylvanian (late } \\
\text { Meramecian-early Atokan)* } \\
\text { CAI=2 } \\
\text { [81MD57C; 38368-PC] } \\
\end{array}$ & Nuka Formation & $\begin{array}{l}\text { Gray, very coarse grained, crinoidal limestone, adjacent to arkosic } \\
\text { limestone. } \\
\text { Fossil loc. } 7 \text { in Mayfield and others (1987). }\end{array}$ \\
\hline $\begin{array}{l}166 \\
\text { KRA (Eli plate) }\end{array}$ & \begin{tabular}{|l|} 
Noatak D-6 \\
$67^{\circ} 51^{\prime} 18^{\prime \prime} /$ \\
$164^{\circ} 32^{\prime} 54^{\prime \prime}$ \\
\end{tabular} & $\begin{array}{l}\text { early Early Mississippian (Kinderhookian) } \\
\text { CAI=1 } \\
\text { [81EK149E; 28588-PC] } \\
\end{array}$ & Utukok Formation & $\begin{array}{l}\text { Sandy limestone. } \\
\text { Conodonts indicate very shallow water environment. } \\
\text { Fossil loc. } 3 \text { in Mayfield and others (1987). } \\
\end{array}$ \\
\hline 168 & \begin{tabular}{|l|} 
Noatak D-4 \\
$67^{\circ} 55^{\prime} 18^{\prime \prime} /$ \\
$163^{\circ} 47^{\prime} 42^{\prime \prime}$ \\
\end{tabular} & $\begin{array}{l}\text { early Late Devonian (early Frasnian) } \\
\text { CAI-2.5-3 } \\
\text { [81EK152; 10461-SD] }\end{array}$ & Devonian carbonate rocks & Silty, terrigenous limestone. \\
\hline
\end{tabular}


Table 13. Conodont samples from the Noatak quadrangle-Continued.

[All faunas identified by A.G. Harris. ${ }^{+}$Structural unit determined by L.E. Young. All other structural and stratigraphic units as reported by C.F. Mayfield and co-workers. EMA, Endicott Mountains allochthon; KRA, Kelly River allochthon; NRA, Nuka Ridge allochthon; PCA, Picnic Creek allochthon. CAI, conodont color alteration index. Letters in field number refer to collector: EK, I. Ellersieck; MD, C.F. Mayfield; and TR, I.L. Tailleur. *, age revised by A.G. Harris in 2000. Lithologic data under remarks are field descriptions unless otherwise indicated; thin section observations by J.A. Dumoulin. No., number; loc., locality

\begin{tabular}{|c|c|c|c|c|}
\hline $\begin{array}{l}\text { Locality No., } \\
\text { structural unit }\end{array}$ & $\begin{array}{l}\text { Quadrangle, } \\
\text { latitude n./ } \\
\text { longitude w. }\end{array}$ & $\begin{array}{l}\text { Age of conodont fauna and CAI } \\
\text { [field No.; USGS collection No.] }\end{array}$ & Stratigraphic unit & Remarks \\
\hline 169 & \begin{tabular}{|l|} 
Noatak D-4 \\
$67^{\circ} 52^{\prime} 48^{\prime \prime} /$ \\
$163^{\circ} 39^{\prime} 48^{\prime \prime}$ \\
\end{tabular} & \begin{tabular}{|l|} 
Late Devonian-Mississippian \\
CAI $=2$ \\
[81EK2A]
\end{tabular} & Uncertain & Black, limy shale and limestone turbidites(?) \\
\hline 170 & \begin{tabular}{|l|} 
Noatak D-3 \\
$67^{\circ} 57^{\prime} 24^{\prime \prime} /$ \\
$163^{\circ} 24^{\prime} 00^{\prime \prime}$
\end{tabular} & $\begin{array}{l}\text { Late Devonian-Early Mississippian* } \\
\text { CAI }=2.5 \\
\text { [81MD3] }\end{array}$ & Devonian carbonate rocks & Limestone. \\
\hline $\begin{array}{ll}171 \\
\text { KRA }\end{array}$ & $\begin{array}{l}\text { Noatak D-3 } \\
67^{\circ} 54^{\prime} 18^{\prime \prime} / \\
163^{\circ} 23^{\prime} 30^{\prime \prime}\end{array}$ & $\begin{array}{l}\text { Mississippian* } \\
\text { CAI }=2.5 \\
{[81 \mathrm{MD} 32 \mathrm{~A}]}\end{array}$ & $\begin{array}{l}\text { Lisburne Group } \\
\text { (Kogruk Formation?) }\end{array}$ & Limestone with minor black chert. \\
\hline $\begin{array}{ll}173 \\
\text { EMA }\end{array}$ & \begin{tabular}{|l|} 
Noatak D-3 \\
$67^{\circ} 45^{\prime} 35^{\prime \prime} /$ \\
$163^{\circ} 06^{\prime} 39.7^{\prime \prime}$ \\
\end{tabular} & $\begin{array}{l}\text { Late Devonian-Early Mississippian } \\
\text { CAI=5 } \\
\text { [81TR51] }\end{array}$ & Lisburne Group & Black shale with minor limestone and chert. \\
\hline $\begin{array}{l}174 \\
\text { EMA }(\text { Key } \\
\text { Creek plate) }\end{array}$ & $\begin{array}{l}\text { Noatak D-3 } \\
67^{\circ} 51^{\prime} 24 " / \\
163^{\circ} 04^{\prime} 06^{\prime \prime}\end{array}$ & $\begin{array}{l}\text { early Early Mississippian (middle-late Kinderhookian)* } \\
\text { CAI }=4-4.5 \\
\text { [81EK100A; 28587-PC] }\end{array}$ & Kayak Shale & $\begin{array}{l}\text { Fauna includes Bispathodus stabilis (Branson and Mehl) } \\
1 \text { Pa and } 1 \text { Sc elements [pl. 3, figs. 9, 10] } \\
\text { Sandy limestone. } \\
\text { Very shallow-water species association. }\end{array}$ \\
\hline $\begin{array}{ll}176 \\
\text { EMA }\end{array}$ & $\begin{array}{l}\text { Noatak C-4 } \\
67^{\circ} 40^{\prime} 08.4^{\prime \prime} / \\
163^{\circ} 50^{\prime} 21.2^{\prime \prime}\end{array}$ & $\begin{array}{l}\text { Late Devonian (Famennian)-Late Mississippian (early Chester) } \\
\text { CAI=5 } \\
\text { [81TR76C] }\end{array}$ & $\begin{array}{l}\text { Lisburne Group } \\
\text { (Kuna Formation?) }\end{array}$ & Black, graphitic limestone. \\
\hline $\begin{array}{l}179 \\
\text { EMA }\end{array}$ & \begin{tabular}{|l|} 
Noatak C-4 \\
$67^{\circ} 34^{\prime} 42^{\prime \prime} /$ \\
$163^{\circ} 54^{\prime} 30^{\prime \prime}$
\end{tabular} & $\begin{array}{l}\text { Early Mississippian } \\
\text { CAI }=5 \\
\text { [81TR81F; 28369-PC] }\end{array}$ & Kayak Shale & Limestone. \\
\hline $\begin{array}{l}180 \\
\text { EMA? }\end{array}$ & \begin{tabular}{|l|} 
Noatak C-3 \\
$67^{\circ} 32^{\prime} 48^{\prime \prime} /$ \\
$163^{\circ} 23^{\prime} 30^{\prime \prime}$
\end{tabular} & $\begin{array}{l}\text { Late Devonian-Early Mississippian } \\
\text { CAI }=5 \\
{[81 \text { MD49] }}\end{array}$ & Devonian carbonate rocks & Limestone from small outcrop surrounded by tundra. \\
\hline \multirow[t]{2}{*}{$\begin{array}{l}181 \\
\text { EMA? }\end{array}$} & \multirow[t]{2}{*}{$\begin{array}{l}\text { Noatak C-3 } \\
67^{\circ} 31^{\prime} 06 " / \\
163^{\circ} 26^{\prime} 06^{\prime \prime}\end{array}$} & $\begin{array}{l}\text { Silurian-Triassic } \\
\text { CAI }=5 \\
{[81 \mathrm{EK} 105 \mathrm{~B}]}\end{array}$ & Devonian carbonate rocks & White limestone. \\
\hline & & $\begin{array}{l}\text { early Late Devonian (Frasnian) } \\
\text { CAI=4.5-5 } \\
{[81 \mathrm{EK} 105 \mathrm{C} ; 10451-\mathrm{SD}]}\end{array}$ & Devonian carbonate rocks & $\begin{array}{l}\text { Silty limestone interbedded with limy quartzite and phyllite. } \\
\text { Megafossils in this sample, identified by J.T. Dutro, Jr., include } \\
\text { molds and casts of the brachiopod Spinatrypa sp., thamnoporoid } \\
\text { corals, and the horn coral Macgeea sp. } \\
\text { These strata correlate lithologically and faunally with limestone of } \\
\text { the Nakolik River (map unit Dnl) of Karl and others (1989). }\end{array}$ \\
\hline $\begin{array}{ll}182 \\
\text { EMA }\end{array}$ & \begin{tabular}{|l|} 
Noatak C-3 \\
$67^{\circ} 31^{\prime} 00^{\prime \prime} /$ \\
$163^{\circ} 28^{\prime} 06^{\prime \prime}$
\end{tabular} & $\begin{array}{l}\text { late Middle-early early Late Devonian (Givetian-early Frasnian) } \\
\text { CAI }=5-5.5 \\
\text { [SPL NO 93943; 10865-SD] }\end{array}$ & Devonian carbonate rocks & $\begin{array}{l}\text { Massive limestone from near top of thin carbonate unit that } \\
\text { conformably underlies the Hunt Fork Shale, basal unit of the } \\
\text { Endicott Group (Moore and others, 1986). } \\
\text { These strata correlate lithologically and faunally with limestone of } \\
\text { the Nakolik River (map unit Dnl) of Karl and others (1989). } \\
4.1 \text { kg of rock was processed. } \\
\text { Sample collected by D. Moore, I.L. Tailleur, and L.E. Young. }\end{array}$ \\
\hline
\end{tabular}


Table 13. Conodont samples from the Noatak quadrangle-Continued.

[All faunas identified by A.G. Harris. ${ }^{+}$Structural unit determined by L.E. Young. All other structural and stratigraphic units as reported by C.F. Mayfield and co-workers. EMA, Endicott Mountains allochthon; KRA, Kelly River allochthon; NRA, Nuka Ridge allochthon; PCA, Picnic Creek allochthon. CAI, conodont color alteration index. Letters in field number refer to collector: EK, I. Ellersieck; MD, C.F. Mayfield; and TR, I.L. Tailleur. *, age revised by A.G. Harris in 2000. Lithologic data under remarks are field descriptions unless otherwise indicated; thin section observations by J.A. Dumoulin. No., number; loc., locality

\begin{tabular}{|c|c|c|c|c|}
\hline $\begin{array}{l}\text { Locality No., } \\
\text { structural unit }\end{array}$ & $\begin{array}{l}\text { Quadrangle, } \\
\text { latitude } \mathrm{n} . / \\
\text { longitude w. }\end{array}$ & $\begin{array}{l}\text { Age of conodont fauna and CAI } \\
\text { [field No.; USGS collection No.] }\end{array}$ & Stratigraphic unit & Remarks \\
\hline $\begin{array}{l}183 \\
\text { EMA? }\end{array}$ & $\begin{array}{l}\text { Noatak B-3 } \\
67^{\circ} 26^{\prime} 48^{\prime \prime} / \\
163^{\circ} 17^{\prime} 30^{\prime \prime}\end{array}$ & $\begin{array}{l}\text { Late Devonian-Early Mississippian* } \\
\text { CAI }=5 \\
\text { [81MD46] }\end{array}$ & Devonian carbonate rocks & Gray limestone. \\
\hline $\begin{array}{l}184 \\
\text { NRA }\end{array}$ & \begin{tabular}{|l|} 
Noatak C-1 \\
$67^{\circ} 35^{\prime} 12^{\prime \prime} /$ \\
$162^{\circ} 17^{\prime} 00^{\prime \prime}$
\end{tabular} & $\begin{array}{l}\text { Silurian-Permian } \\
\text { CAI }=4 \\
{[81 \mathrm{EK} 208 \mathrm{~A}]}\end{array}$ & Devonian carbonate rocks & Brown-weathering, silty to sandy limestone. \\
\hline 185 & \begin{tabular}{|l|} 
Noatak C-1 \\
$67^{\circ} 35^{\prime} 30^{\prime \prime} /$ \\
$162^{\circ} 05^{\prime} 18^{\prime \prime}$
\end{tabular} & $\begin{array}{l}\text { Silurian-Middle Devonian, likely Middle Devonian* } \\
\text { CAI }=5-5.5 \\
\text { [81MD72D; 10494-SD] }\end{array}$ & $\begin{array}{l}\text { Devonian carbonate rocks } \\
\text { (Baird Group?) }\end{array}$ & Dark-weathering dolomitic limestone with local black chert nodules \\
\hline 186 & \begin{tabular}{|l|} 
Noatak B-1 \\
$67^{\circ} 25^{\prime} 00^{\prime \prime} /$ \\
$162^{\circ} 05^{\prime} 00^{\prime \prime}$ \\
\end{tabular} & \begin{tabular}{|l|} 
Late Silurian-Middle Devonian \\
CAI=5 \\
[81MD83B; 10492-SD] \\
\end{tabular} & $\begin{array}{l}\text { Devonian carbonate rocks } \\
\text { (Baird Group?) }\end{array}$ & Carbonate with black chert. \\
\hline 187 & \begin{tabular}{|l|} 
Noatak A-3 \\
$67^{\circ} 11^{\prime} 42^{\prime \prime} /$ \\
$163^{\circ} 20^{\prime} 42^{\prime \prime}$
\end{tabular} & \begin{tabular}{|l|} 
Silurian-Triassic \\
CAI $=4$ \\
[81MD67E]
\end{tabular} & $\begin{array}{l}\text { Devonian carbonate rocks } \\
\text { (Baird Group?) }\end{array}$ & Partly metamorphosed limestone. \\
\hline 188 & \begin{tabular}{|l|} 
Noatak A-3 \\
$67^{\circ} 10^{\prime} 18^{\prime \prime} /$ \\
$163^{\circ} 02^{\prime} 30^{\prime \prime}$ \\
\end{tabular} & \begin{tabular}{|l|} 
Silurian-Permian \\
CAI $=4.5$ \\
{$[81 \mathrm{EK} 218 \mathrm{~B}]$} \\
\end{tabular} & $\begin{array}{l}\text { Devonian carbonate rocks } \\
\text { (Baird Group?) }\end{array}$ & Dark gray dolostone and limestone with chert nodules. \\
\hline 189 & \begin{tabular}{|l|} 
Noatak A-1 \\
$67^{\circ} 09^{\prime} 52.6^{\prime \prime} /$ \\
$162^{\circ} 05^{\prime} 17^{\prime \prime}$ \\
\end{tabular} & $\begin{array}{l}\text { Early Mississippian } \\
\text { CAI }=5 \\
\text { [81TR179] }\end{array}$ & Kayak Shale & $\begin{array}{l}\text { Sandy to silty carbonate lens in Kayak Shale. } \\
\text { Heavy-mineral concentrate includes phosphatized gastropod } \\
\text { steinkerns. }\end{array}$ \\
\hline
\end{tabular}


Table 14. Radiolarian samples from the De Long Mountains quadrangle

[All faunas identified by C.D. Blome unless otherwise indicated. Structural units determined by L.E. Young. EMA, Endicott Mountains allochthon; KRA, Kelly River allochthon; PCA, Picnic Creek allochthon. Letters in field number refer to collector: AD, J.A. Dumoulin; AK, J.S. Kelley; and JS, J.M. Schmidt. DDH, diamond drill hole. Interval thicknesses in drill holes are drilled thicknesses and have not been corrected for dip of bedding. All drill hole samples collected by J.A. Dumoulin and (or) A.G. Harris unless otherwise indicated. Lithologic data under remarks are field descriptions unless otherwise indicated; thin section observations by J.A. Dumoulin. No., number; loc., locality]

\begin{tabular}{|c|c|c|c|c|}
\hline $\begin{array}{l}\text { Locality No., } \\
\text { structural unit }\end{array}$ & $\begin{array}{l}\text { Quadrangle, } \\
\text { latitude n./ } \\
\text { longitude w. }\end{array}$ & $\begin{array}{c}\text { Radiolarian fauna } \\
\text { [field No.; USGS collection No.] }\end{array}$ & Age & Remarks \\
\hline \multirow[t]{3}{*}{$\begin{array}{l}5 \\
\text { KRA (Wulik } \\
\text { Peaks plate) }\end{array}$} & \multirow[t]{3}{*}{\begin{tabular}{|l|} 
De Long Mtns. \\
B-3 \\
$68^{\circ} 17^{\prime} 37^{\prime \prime} /$ \\
$163^{\circ} 22^{\prime} 04^{\prime \prime}$
\end{tabular}} & $\begin{array}{l}\text { One possible tumidaspine belonging to the Late Triassic } \\
\text { radiolarian family Capnuchosphaeridae DeWever (1979), } \\
\text { emend. Pessagno (1979), emend. Blome (1983) } \\
\text { [00AD4D; DR 2526] }\end{array}$ & ?Late Triassic & $\begin{array}{l}\text { Sample from upper subunit of Siksikpuk Formation(?) (Etivluk } \\
\text { Group) overlying Kogruk Formation; see table } 6 \text { for conodont } \\
\text { sample from Kogruk at this locality. Triassic age is unlikely for } \\
\text { Siksikpuk. } \\
\text { Greenish-gray chert. Thin section shows locally well-preserved } \\
\text { radiolarians filled with microcrystalline quartz and (or) chalcedony } \\
\text { in matrix of cryptocrystalline to fine-crystalline chert with veins of } \\
\text { barite, a few phosphate(?) clasts, and minor dolomite. } \\
\text { Loc. } 1 \text { (fig. 2) of Dumoulin and others (2004). }\end{array}$ \\
\hline & & $\begin{array}{l}\text { Corum perfectum Blome } \\
\text { Muelleritortis cochleata cochleata (Nakaseko and Nishimura) } \\
\text { Pseudostylosphaera coccostyle (Rüst) } \\
\text { Pseudostylosphaera japonica (Nakaseko and Nishimura) } \\
\text { Pseudostylosphaera nazarovi (Kozur and Mostler) } \\
\text { Triassocampe sp. } \\
\text { [00AD4E; DR 2516] }\end{array}$ & $\begin{array}{l}\text { Middle Triassic (middle to } \\
\text { late Ladinian). }\end{array}$ & $\begin{array}{l}\text { Sample from middle subunit of Otuk Formation (Etivluk Group), } \\
\text { below recessive marker. } \\
\text { Gray chert. Thin section shows abundant, locally well-preserved } \\
\text { radiolarians in matrix of cryptocrystalline to fine-crystalline chert, } \\
\text { with veins of barite, scattered carbonate crystals, and rare } \\
\text { phosphatic bioclasts. }\end{array}$ \\
\hline & & $\begin{array}{l}\text { Capnuchosphaera sp. } \\
\text { Capnuchosphaera sp. aff. C. deweveri DeWever } \\
\text { Capnuchosphaera tricornis DeWever } \\
\text { Paleosaturnalis sp. (fragment) } \\
\text { Pseudostylosphaera helicatum (Nakaseko and Nishimura) } \\
\text { Pseudostylosphaera japonica (Nakaseko and Nishimura) } \\
\text { Abundant Pseudostylosphaera sp. } \\
\text { [00AD4F; DR 2517] }\end{array}$ & $\begin{array}{l}\text { early Late Triassic (early to } \\
\text { middle Carnian) }\end{array}$ & $\begin{array}{l}\text { Sample from middle(?) subunit of Otuk Formation, above recessive } \\
\text { marker. } \\
\text { Medium-gray and white chert; thin section shows abundant } \\
\text { radiolarians in matrix of cryptocrystalline to fine-crystalline chert, } \\
\text { with veins of barite, scattered carbonate crystals, and rare } \\
\text { phosphatic bioclasts. }\end{array}$ \\
\hline $\begin{array}{l}33 \\
\text { PCA (Wulik } \\
\text { plate) }\end{array}$ & \begin{tabular}{|l|} 
De Long Mtns. \\
B-2 \\
$68^{\circ} 24^{\prime} 45^{\prime \prime} /$ \\
$162^{\circ} 37^{\prime} 21.5^{\prime \prime}$
\end{tabular} & $\begin{array}{l}\text { Bipedis acrostylus Bragin } \\
\text { Betraccium sp. aff. B. perilense Carter } \\
\text { Canutus? ingrahamensis Carter } \\
\text { Ferresium sp. } \\
\text { Pantanellium sp. aff. P. fosteri } \text { Pessagno and Blome } \\
\text { All forms very poorly preserved and coated with } \\
\quad \text { manganese/iron stains. } \\
\text { [99AD1D; DR 2490] }\end{array}$ & \begin{tabular}{|l} 
Late Triassic (late Norian or \\
Rhaetian). The ranges of \\
these taxa indicate that the \\
fauna is assignable to either \\
the Betraccium deweveri \\
Zone (late Norian) of \\
Blome (1984) and Blome \\
and others (1988) or the \\
Proparvicingula \\
moniliformis and \\
Globolaxtorum tozeri Zones \\
(Rhaetian) of Carter (1993).
\end{tabular} & $\begin{array}{l}\text { Probable upper subunit of Otuk Formation (Etivluk Group). } \\
\text { Dark-gray- to light-brown-weathering, grayish black siliceous } \\
\text { mudstone in fairly even, } 5 \text { to } 8 \mathrm{~cm} \text { thick beds. Thin section shows } \\
\text { abundant, locally well preserved radiolarians (tests have sharp } \\
\text { edges) and lesser thin "spicules" in a muddy matrix; radiolarian test } \\
\text { shapes fairly diverse. }\end{array}$ \\
\hline
\end{tabular}


Table 14. Radiolarian samples from the De Long Mountains quadrangle-Continued.

[All faunas identified by C.D. Blome unless otherwise indicated. Structural units determined by L.E. Young. EMA, Endicott Mountains allochthon; KRA, Kelly River allochthon; PCA, Picnic Creek allochthon. Letters in field number refer to collector: AD, J.A. Dumoulin; AK, J.S. Kelley; and JS, J.M. Schmidt. DDH, diamond drill hole. Interval thicknesses in drill holes are drilled thicknesses and have not been corrected for dip of bedding. All drill hole samples collected by J.A. Dumoulin and (or) A.G. Harris unless otherwise indicated. Lithologic data under remarks are field descriptions unless otherwise indicated; thin section observations by J.A. Dumoulin. No., number; loc., locality]

\begin{tabular}{|c|c|c|c|c|}
\hline $\begin{array}{l}\text { Locality No., } \\
\text { structural unit }\end{array}$ & $\begin{array}{l}\text { Quadrangle, } \\
\text { latitude n./ } \\
\text { longitude w. }\end{array}$ & $\begin{array}{c}\text { Radiolarian fauna } \\
\text { [field No.; USGS collection No.] }\end{array}$ & Age & Remarks \\
\hline \multirow[t]{2}{*}{$\begin{array}{l}35 \\
\text { PCA (Wulik } \\
\text { plate) }\end{array}$} & \multirow[t]{2}{*}{$\begin{array}{l}\text { De Long Mtns. } \\
\text { B-2 } \\
68^{\circ} 21^{\prime} 56 " \\
162^{\circ} 49^{\prime} 54.5^{\prime \prime}\end{array}$} & $\begin{array}{l}\text { Corum speciosum Blome } \\
\text { Corum regium Kozur and Mostler } \\
\text { Poulpus transitus Kozur and Mostler } \\
\text { Pseudostylosphaera japonica (Nakaseko and Nishimura) } \\
\text { Pseudostylosphaera sp. } \\
\text { All forms poorly preserved } \\
\text { [99AD19A; DR 2525] }\end{array}$ & $\begin{array}{l}\text { early Late Triassic (Carnian, } \\
\text { probably early to middle, } \\
\text { although a few of the taxa } \\
\text { range into the upper } \\
\text { Carnian) }\end{array}$ & $\begin{array}{l}\text { Sample from rubble of Etivluk Group (Otuk Formation?) directly } \\
\text { adjacent to black siliceous mudstone (uppermost Lisburne } \\
\text { Group?). Siksikpuk Formation appears to be missing here. See } \\
\text { table } 4 \text { for Lisburne conodont sample from this locality. } \\
\text { Medium-gray-green chert. Thin section shows scattered to abundant } \\
\text { radiolarians in matrix of cryptocrystalline to fine-crystalline chert; } \\
\text { radiolarians filled with fine-crystalline quartz (locally chalcedony) } \\
\text { some sharp test edges. }\end{array}$ \\
\hline & & $\begin{array}{l}\text { Eptingium sp. cf. E. manfredi Dumitrica } \\
\text { Triassocampe deweveri (Nakaseko and Nishimura) } \\
\text { Triassocampe sp. } \\
\text { Vinassaspongus erendili Tekin } \\
\text { Vinassaspongus subsphaericus Kozur and Mostler } \\
\text { [99AD19B; DR 2520] }\end{array}$ & $\begin{array}{l}\text { late Middle Triassic (probably } \\
\text { middle to late Ladinian) }\end{array}$ & $\begin{array}{l}\text { Subcrop of Etivluk Group (Otuk Formation) chert a few feet } \\
\text { southeast of 99AD19A. } \\
\text { Green chert. Thin section shows scattered to abundant radiolarians in } \\
\text { matrix of cryptocrystalline to fine-crystalline chert; radiolarians } \\
\text { filled with fine-crystalline quartz; many sharp test edges and some } \\
\text { preserved rim structure. }\end{array}$ \\
\hline $\begin{array}{l}41 \\
\text { EMA (Red Dog } \\
\text { plate) }\end{array}$ & $\begin{array}{l}\text { De Long Mtns. } \\
\text { A-2 } \\
68^{\circ} 14^{\prime} 22^{\prime \prime} / \\
162^{\circ} 54^{\prime} 33.8^{\prime \prime}\end{array}$ & $\begin{array}{l}\text { Paleoxyphostylus variospina Won } \\
\text { Scharfenbergia tailleurense Holdsworth and Murchey } \\
\text { ?Scharfenbergia ruestae (Ormiston and Lane) } \\
\text { No albaillellids found, despite lots of picking. Is there } \\
\text { something odd about this facies? } \\
\text { All forms poorly preserved } \\
\text { [98AD10B; DR 2452] }\end{array}$ & $\begin{array}{l}\text { late Late Mississippian-early } \\
\text { Middle Pennsylvanian } \\
\text { (Chesterian to Morrowan). } \\
\text { Faunal group 2 of } \\
\text { Holdsworth and Murchey } \\
\text { (1988) and Assemblage } \\
\text { Zone } 5 \text { (Scharfenbergia } \\
\text { tailleurense assemblage) of } \\
\text { Murchey (1990). } \\
\text { Constraints from conodonts } \\
\text { at this locality restrict age } \\
\text { to early Chesterian. }\end{array}$ & $\begin{array}{l}\text { Chert-rich upper part of Ikalukrok unit, Kuna Formation. Calcareous } \\
\text { radiolarite bed several meters below this sample was collected for } \\
\text { conodonts (98AD10A); see table } 1 \text { for conodont data from this } \\
\text { locality. } \\
\text { Black chert in even beds, } 1 \text { to } 15 \mathrm{~cm} \text { thick; faint millimeter-scale } \\
\text { color laminae. Thin section shows abundant, well preserved } \\
\text { radiolarians (sharp edges) in a muddy, cherty matrix. Most } \\
\text { radiolarians filled with chalcedony; a few with barite. } \\
\text { Loc. } 3 \text { (fig. 2) of Dumoulin and others (2004). }\end{array}$ \\
\hline
\end{tabular}


Table 14. Radiolarian samples from the De Long Mountains quadrangle-Continued.

[All faunas identified by C.D. Blome unless otherwise indicated. Structural units determined by L.E. Young. EMA, Endicott Mountains allochthon; KRA, Kelly River allochthon; PCA, Picnic Creek allochthon. Letters in field number refer to collector: AD, J.A. Dumoulin; AK, J.S. Kelley; and JS, J.M. Schmidt. DDH, diamond drill hole. Interval thicknesses in drill holes are drilled thicknesses and have not been corrected for dip of bedding. All drill hole samples collected by J.A. Dumoulin and (or) A.G. Harris unless otherwise indicated. Lithologic data under remarks are field descriptions unless otherwise indicated; thin section observations by J.A. Dumoulin. No., number; loc., locality]

\begin{tabular}{|c|c|c|c|c|}
\hline $\begin{array}{l}\text { Locality No., } \\
\text { structural unit }\end{array}$ & $\begin{array}{l}\text { Quadrangle, } \\
\text { latitude n./ } \\
\text { longitude w. }\end{array}$ & $\begin{array}{c}\text { Radiolarian fauna } \\
\text { [field No.; USGS collection No.] }\end{array}$ & Age & Remarks \\
\hline $\begin{array}{l}61 \\
\text { KRA (Wulik } \\
\text { Peaks plate) }\end{array}$ & $\begin{array}{l}\text { De Long Mtns. } \\
\text { A-2 } \\
68^{\circ} 11^{\prime} 07^{\prime \prime} / \\
163^{\circ} 10^{\prime} 44.4^{\prime \prime}\end{array}$ & $\begin{array}{l}\text { Entactinia itsukaichiensis Sashida and Tonishi } \\
\text { Entactinia modesta } \text { Sashida and Tonishi } \\
\text { Follicucullus sp. aff. P. monacanthus Ishiga and Imoto } \\
\text { Latentifistula } \text { sp. } \\
\text { Nazarovella } \text { sp. (fragment) } \\
\text { Pseudoalbaillella sp. aff. P. longicornis Ishiga and Imoto } \\
\text { All forms poorly preserved } \\
\text { [98JS7A; DR2485 and } 86]\end{array}$ & $\begin{array}{l}\text { late Early-middle Middle } \\
\text { Permian (middle } \\
\text { Leonardian-middle } \\
\text { Guadalupian). } \\
\text { Fauna assignable to } \\
\text { Pseudoalbaillella globosa } \\
\text { Zone of Ishiga (1986). }\end{array}$ & $\begin{array}{l}\text { Sample from middle(?) subunit of Siksikpuk Formation (Etivluk } \\
\text { Group) overlying Kogruk Formation. Conodont sample 98JS7K, } \\
\text { taken from top of Kogruk slightly to southeast (loc. 62, table 6), } \\
\text { contained late Meramecian-Chesterian conodonts. } \\
\text { Maroon and light-greenish-gray shale and lesser chert. Thin section } \\
\text { consists of abundant radiolarians in a red muddy matrix. } \\
\text { This sample consisted of abundant maroon chert pieces and one large } \\
\text { gray chert piece. The maroon chert (DR 2485) was processed } \\
\text { separately from the gray chert (DR 2486). Preservation of } \\
\text { radiolarians was slightly better in the gray chert and this piece } \\
\text { provided most of the identifiable Permian radiolarians. }\end{array}$ \\
\hline $\begin{array}{l}65 \\
\text { EMA (Red Dog } \\
\text { plate) }\end{array}$ & $\begin{array}{l}\text { De Long Mtns. } \\
\text { A-2 } \\
68^{\circ} 10^{\prime} 02.8^{\prime \prime} / \\
162^{\circ} 52^{\prime} 40^{\prime \prime}\end{array}$ & $\begin{array}{l}\text { Albaillella } \text { sp. aff. A. cartalla Ormiston and Lane (abundant } \\
\text { forms) } \\
\text { Albaillella sp. (same form that is shown in Holdsworth and } \\
\text { Murchey, 1986, pl. 34.1, number 7) } \\
\text { Pylentonema } \text { sp. cf. P. antiqua Deflandre (see fig. 6, nos. 22-23, } \\
\text { Blome, Reed, and Harris, 1998) } \\
\text { Scharfenbergia impella group (Ormiston and Lane) } \\
\text { Triaenosphaera hebes Won (partial test; entactinid with broad } \\
\text { spines) } \\
\text { All forms poorly preserved } \\
\text { [98AD7A; DR 2451] }\end{array}$ & $\begin{array}{l}\text { early Late Mississippian } \\
\text { (Meramecian to lower part } \\
\text { of the Chesterian). Faunal } \\
\text { group 1B of Holdsworth } \\
\text { and Murchey (1988) and } \\
\text { Assemblage Zone } 4 \\
\text { (Scharfenbergia impella gp } \\
\text { - Albaillella sp. aff. A. } \\
\text { cartalla assemblage ) of } \\
\text { Murchey (1990). }\end{array}$ & $\begin{array}{l}\text { Ikalukrok unit of Kuna Formation; Suds prospect. } \\
\text { Dark gray to black chert; hillside rubble crop. Thin section shows } \\
\text { abundant radiolarians that appear well preserved (sharp rims) in a } \\
\text { muddy, cherty matrix. }\end{array}$ \\
\hline $\begin{array}{l}66 \\
\text { EMA (Red Dog } \\
\text { plate) }\end{array}$ & $\begin{array}{l}\text { De Long Mtns. } \\
\text { A-2 } \\
68^{\circ} 10^{\prime} 18^{\prime \prime} / \\
162^{\circ} 50^{\prime} 48^{\prime \prime}\end{array}$ & $\begin{array}{l}\text { Betraccium sp. } \\
\text { Capnuchosphaera sp. aff. C. lenticulata Pessagno } \\
\text { Capnuchosphaera mexicana Pessagno } \\
\text { Capnuchosphaera schenki Blome } \\
\text { Capnuchosphaera smithorum Blome } \\
\text { Capnuchosphaera sp. aff. C. theoloides DeWever } \\
\text { Pseudoheliodiscus viejoensis Pessagno } \\
\text { Sarla vetusta Pessagno } \\
\text { [KE98-28; DR 2487] }\end{array}$ & $\begin{array}{l}\text { Conodonts from this locality } \\
\text { indicate late Middle-Late } \\
\text { Triassic, but radiolarians } \\
\text { restrict age to middle Late } \\
\text { Triassic (late middle-early } \\
\text { late Norian). } \\
\text { Radiolarian fauna assignable } \\
\text { to the Betraccium Zone } \\
\text { (Pantanellium silberlingi } \\
\text { Subzone) of Pessagno and } \\
\text { others (1979) and Blome } \\
\text { and others (1988). } \\
\end{array}$ & $\begin{array}{l}\text { Sample from Otuk Formation (Etivluk Group); see table } 9 \text { for } \\
\text { conodont sample from this locality. } \\
\text { Chert interbedded with very fine grained dolostone. Carbonate } \\
\text { interbeds contain rare thin bivalve shells identified as Monotis(?) } \\
\text { sp. of Late Triassic age by R.B. Blodgett (Oregon State University, } \\
\text { unpublished fossil report, 1999) and yielded a single conodont. }\end{array}$ \\
\hline
\end{tabular}


Table 14. Radiolarian samples from the De Long Mountains quadrangle-Continued.

[All faunas identified by C.D. Blome unless otherwise indicated. Structural units determined by L.E. Young. EMA, Endicott Mountains allochthon; KRA, Kelly River allochthon; PCA, Picnic Creek allochthon. Letters in field number refer to collector: AD, J.A. Dumoulin; AK, J.S. Kelley; and JS, J.M. Schmidt. DDH, diamond drill hole. Interval thicknesses in drill holes are drilled thicknesses and have not been corrected for dip of bedding. All drill hole samples collected by J.A. Dumoulin and (or) A.G. Harris unless otherwise indicated. Lithologic data under remarks are field descriptions unless otherwise indicated; thin section observations by J.A. Dumoulin. No., number; loc., locality]

\begin{tabular}{|c|c|c|c|c|}
\hline $\begin{array}{l}\text { Locality No., } \\
\text { structural unit }\end{array}$ & $\begin{array}{l}\text { Quadrangle, } \\
\text { latitude } \mathrm{n} . / \\
\text { longitude w. }\end{array}$ & $\begin{array}{c}\text { Radiolarian fauna } \\
\text { [field No.; USGS collection No.] }\end{array}$ & Age & Remarks \\
\hline \multirow[t]{3}{*}{$\begin{array}{l}72 \\
\text { EMA (Key } \\
\text { Creek plate) }\end{array}$} & \multirow[t]{3}{*}{$\begin{array}{l}\text { De Long Mtns. } \\
\text { A-2 } \\
68^{\circ} 07^{\prime} 37.8^{\prime \prime} \\
162^{\circ} 49^{\prime} 15.6^{\prime \prime}\end{array}$} & $\begin{array}{l}\text { Belowea variabilis (Ormiston and Lane) } \\
\text { Scharfenbergia ruestae (Ormiston and Lane) } \\
\text { Scharfenbergia sp. (broken arms) } \\
\text { All radiolarians poorly preserved } \\
\text { [00AD20A; DR 2521] }\end{array}$ & $\begin{array}{l}\text { Probably late Late } \\
\text { Mississippian to early } \\
\text { Middle Pennsylvanian } \\
\text { (probably Chesterian- } \\
\text { Morrowan) }\end{array}$ & $\begin{array}{l}\text { Siksikpuk Formation (base of lower subunit) } 0.5 \mathrm{~m} \text { above Kuna } \\
\text { Formation, on southeast side of "Orange Creek." Contact marked } \\
\text { by horizon of mammiform structures with } 40 \mathrm{~cm} \text { of vertical relief. } \\
\text { Sample from } 10 \mathrm{~cm} \text { thick bed of moderate orange weathering, dark } \\
\text { gray (locally slightly olive green) chert. Thin section shows } \\
\text { scattered radiolarians, most filled with chalcedony, in } \\
\text { cryptocrystalline chert matrix; test margins generally sharp and rim } \\
\text { structure locally well preserved. }\end{array}$ \\
\hline & & $\begin{array}{l}\text { Scharfenbergia concentrica (Rüst) } \\
\text { Scharfenbergia ruestae (Ormiston and Lane) } \\
\text { Scharfenbergia tailleurense Holdsworth and Murchey } \\
\text { Scharfenbergia sp. } \\
\text { No albaillelids found } \\
\text { All forms poorly preserved } \\
\text { [00AD20B; DR 2515] }\end{array}$ & $\begin{array}{l}\text { late Late Mississippian to } \\
\text { early Middle Pennsylvanian } \\
\text { (Chesterian to Morrowan) }\end{array}$ & $\begin{array}{l}\text { Upper Kuna Formation, just below contact with Siksikpuk } \\
\text { Formation. Upper Kuna here consists of } \sim 8 \mathrm{~m} \text { of chert above black } \\
\text { shale. } \\
\text { Medium-dark-gray to grayish black chert in } 2 \text { to } 5 \mathrm{~cm} \text { thick beds. } \\
\text { Thin section shows abundant radiolarians, filled with chalcedony } \\
\text { or polycrystalline quartz, in dark muddy matrix; test margins } \\
\text { generally sharp and rim structure locally well preserved; some } \\
\text { siliceous sponge spicules. }\end{array}$ \\
\hline & & $\begin{array}{l}\text { Belowea variabilis (Ormiston and Lane) } \\
\text { Scharfenbergia ruestae (Ormiston and Lane) } \\
\text { [00AD20C; DR 2524] }\end{array}$ & $\begin{array}{l}\text { late Late Mississippian to } \\
\text { early Middle Pennsylvanian } \\
\text { (Chesterian to Morrowan) }\end{array}$ & $\begin{array}{l}\text { Siksikpuk Formation, lower subunit, } 0.5 \mathrm{~m} \text { above 00AD20A. } \\
\text { Medium-gray to olive-gray chert, weathers moderate brown. Thin } \\
\text { section shows scattered to abundant radiolarians filled with } \\
\text { chalcedony or polycrystalline quartz; some fairly well-preserved } \\
\text { with sharp margins. }\end{array}$ \\
\hline \multirow[t]{2}{*}{$\begin{array}{l}88 \\
\text { EMA } \\
\text { (Wolverine } \\
\text { Creek plate) }\end{array}$} & \multirow[t]{2}{*}{$\begin{array}{l}\text { De Long Mtns. } \\
\text { A-2 } \\
68^{\circ} 01^{\prime} 12^{\prime \prime} / \\
162^{\circ} 45^{\prime} 00^{\prime \prime}\end{array}$} & $\begin{array}{l}\text { One cast of ?Albaillella } \text { sp. } \\
\text { Several poorly preserved spumellarians } \\
\text { [98AD18B; DR 2453] }\end{array}$ & Questionable Paleozoic & $\begin{array}{l}\text { Sample from section of unnamed deep-water facies of the Lisburne } \\
\text { Group underlying the Kogruk Formation on Anxiety Ridge. } \\
\text { Medium gray, fine-grained carbonate rock, in part replaced by(?) } \\
\text { black chert. Thin section is fine-grained carbonate (dolomite?) with } \\
\text { irregular zones of chert, relict peloids, and skeletal grains; bioclasts } \\
\text { include spicules and minor but well-preserved radiolarians (sharp } \\
\text { rims). } \\
\text { Loc. } 22 \text { (fig. 2) of Dumoulin and others (2004). }\end{array}$ \\
\hline & & $\begin{array}{l}2 \text { flattened poorly preserved casts of Albaillella } \text { sp. } \\
\text { [98AD18F; DR 2454] }\end{array}$ & \begin{tabular}{|l} 
Paleozoic (Devonian to \\
Middle Pennsylvanian).
\end{tabular} & $\begin{array}{l}\text { Sample from section of unnamed deep-water facies of the Lisburne } \\
\text { Group underlying the Kogruk Formation on Anxiety Ridge, } \sim 0.2 \\
\text { km south of sample 98AD18B. } \\
\text { Black mudstone rubble, weathers into dull, blocky slabs; interbeds of } \\
\text { brownish gray weathering, black fetid dolostone. Thin section is } \\
\text { partly silicified mudstone with locally abundant siliceous bioclasts } \\
\text { including radiolarians and spicules. }\end{array}$ \\
\hline $\begin{array}{l}100 \\
\text { EMA (Key } \\
\text { Creek plate) }\end{array}$ & $\begin{array}{l}\text { De Long Mtns. } \\
\text { B-1 } \\
68^{\circ} 19^{\prime} 00^{\prime \prime} / \\
162^{\circ} 16^{\prime} 35^{\prime \prime}\end{array}$ & $\begin{array}{l}\text { Poorly preserved casts of radiolarians (simple spheres) } \\
\text { [00AD14A; DR 2522] }\end{array}$ & Unknown & $\begin{array}{l}\text { Upper Kuna Formation, } 20 \mathrm{~cm} \text { below contact with the Siksikpuk } \\
\text { Formation; contact here marked by horizon of mammiform } \\
\text { structures with }>5 \mathrm{~cm} \text { of vertical relief. } \\
\text { Black chert in blocky } 5 \text { - to } 8 \text {-cm-thick beds. Thin section shows } \\
\text { abundant, locally very well preserved radiolarians (with rim } \\
\text { structure and sharp test margins) in dark muddy matrix. }\end{array}$ \\
\hline
\end{tabular}


Table 14. Radiolarian samples from the De Long Mountains quadrangle-Continued.

[All faunas identified by C.D. Blome unless otherwise indicated. Structural units determined by L.E. Young. EMA, Endicott Mountains allochthon; KRA, Kelly River allochthon; PCA, Picnic Creek allochthon. Letters in field number refer to collector: AD, J.A. Dumoulin; AK, J.S. Kelley; and JS, J.M. Schmidt. DDH, diamond drill hole. Interval thicknesses in drill holes are drilled thicknesses and have not been corrected for dip of bedding. All drill hole samples collected by J.A. Dumoulin and (or) A.G. Harris unless otherwise indicated. Lithologic data under remarks are field descriptions unless otherwise indicated; thin section observations by J.A. Dumoulin. No., number; loc., locality]

\begin{tabular}{|c|c|c|c|c|}
\hline $\begin{array}{l}\text { Locality No., } \\
\text { structural unit }\end{array}$ & $\begin{array}{l}\text { Quadrangle, } \\
\text { latitude n./ } \\
\text { longitude w. }\end{array}$ & $\begin{array}{c}\text { Radiolarian fauna } \\
\text { [field No.; USGS collection No.] }\end{array}$ & Age & Remarks \\
\hline \multirow[t]{7}{*}{$\begin{array}{l}107 \\
\text { EMA (Key } \\
\text { Creek plate) }\end{array}$} & \multirow[t]{7}{*}{$\begin{array}{l}\text { De Long Mtns. } \\
\text { B-1 } \\
68^{\circ} 15^{\prime} 31.4^{\prime \prime} \\
162^{\circ} 13^{\prime} 20^{\prime \prime}\end{array}$} & $\begin{array}{l}\text { ?Scharfenbergia ruestae (Ormiston and Lane) } \\
\text { Scharfenbergia tailleurense Holdsworth and Murchey } \\
\text { [97AK74B and E] }\end{array}$ & \multirow[t]{2}{*}{$\begin{array}{l}\text { Radiolarians indicate an age } \\
\text { of, most likely, late Late } \\
\text { Mississippian to early } \\
\text { Middle Pennsylvanian } \\
\text { (Chesterian to Morrowan). } \\
\text { Conodonts from this locality } \\
\text { (97AK74F, } 40 \mathrm{ft} \text { above } \\
\text { base of section) indicate } \\
\text { that these strata are no } \\
\text { younger than early } \\
\text { Chesterian (see table } 3 \text { ). }\end{array}$} & $\begin{array}{l}\text { Samples from Kuna Formation; part of } 158 \text {-ft-thick measured section } \\
\text { of upper Kuna and lower Etivluk Group (Siksikpuk Formation). } \\
\text { We consider the contact between the two units to occur } \sim 88 \mathrm{ft} \\
\text { above the base of the section, at the top of a horizon of } \\
\text { mammiform structures as much as } 1 \mathrm{ft} \text { high. See table } 3 \mathrm{for} \\
\text { conodont samples from this locality. } \\
\text { 74B taken } \sim 16 \mathrm{ft} \text { above base of section ( } 72 \mathrm{ft} \text { below top of Kuna) } \\
\text { from medium-dark-gray to black chert in nodular beds } 0.5 \text { to } 10 \mathrm{~cm} \\
\text { thick with shale partings. } \\
74 \mathrm{E} \text { taken } \sim 34 \mathrm{ft} \text { above base of section ( } 54 \mathrm{ft} \text { below top of Kuna) } \\
\text { from similar chert in beds to } 5 \mathrm{~cm} \text { thick with black shale partings. } \\
\text { All faunas from this section identified by K. Reed (unpublished fossil } \\
\text { report, 1998). } \\
\text { Loc. } 6 \text { (app. 1) of Dumoulin and others (2004). }\end{array}$ \\
\hline & & $\begin{array}{l}\text { ?Scharfenbergia ruestae (Ormiston and Lane) } \\
\text { [97AK74D] }\end{array}$ & & $\begin{array}{l}\text { Sample } \sim 26 \mathrm{ft} \text { above base of section and } \sim 62 \mathrm{ft} \text { below top of Kuna } \\
\text { Formation. } \\
\text { Medium-dark-gray to black chert in nodular beds } 0.5 \text { to } 10 \mathrm{~cm} \text { thick } \\
\text { with shale partings. }\end{array}$ \\
\hline & & $\begin{array}{l}\text { ?Scharfenbergia ruestae (Ormiston and Lane) } \\
\text { [97AK } 74 \mathrm{G} \text { and J] }\end{array}$ & $\begin{array}{l}\text { late Late Mississippian to } \\
\text { early Middle Pennsylvanian } \\
\text { (Chesterian to Morrowan). }\end{array}$ & $\begin{array}{l}\text { Samples from Kuna Formation, from medium-dark-gray to black } \\
\text { chert in nodular beds to } 10 \mathrm{~cm} \text { thick with black shale partings. } \\
74 \mathrm{G} \text { taken } \sim 45 \mathrm{ft} \text { above base of section ( } 43 \mathrm{ft} \text { below top of Kuna). } \\
74 \mathrm{~J} \text { taken } \sim 63 \mathrm{ft} \text { above base of section ( } 25 \mathrm{ft} \text { below top of Kuna). }\end{array}$ \\
\hline & & $\begin{array}{l}\text { Glassy spheres (probable casts of spumellarians) } \\
\text { [97AK74H and I] }\end{array}$ & $\begin{array}{l}\text { late Late Mississippian to } \\
\text { early Middle Pennsylvanian } \\
\text { (Chesterian to Morrowan), } \\
\text { based on age of underlying } \\
\text { and overlying samples. }\end{array}$ & $\begin{array}{l}\text { Samples from Kuna Formation, from medium-dark-gray to black } \\
\text { chert in even beds } 2 \text { to } 15 \mathrm{~cm} \text { thick with black shale partings. } \\
74 \mathrm{H} \text { taken } \sim 50 \mathrm{ft} \text { above base of section ( } 38 \mathrm{ft} \text { below top of Kuna). } \\
74 \mathrm{I} \text { taken } \sim 55 \mathrm{ft} \text { above base of section ( } 33 \mathrm{ft} \text { below top of Kuna). }\end{array}$ \\
\hline & & $\begin{array}{l}\text { "late scharfenbergiid" form of Holdsworth and Murchey } \\
\text { [97AK74K and L] }\end{array}$ & \multirow[t]{3}{*}{$\begin{array}{l}\text { late Late Mississippian to } \\
\text { early Middle Pennsylvanian } \\
\text { (Chesterian to Morrowan). }\end{array}$} & $\begin{array}{l}\text { Samples from Kuna Formation, from medium-dark-gray to black } \\
\text { chert in beds } 1 \text { to } 10 \mathrm{~cm} \text { thick with shale partings. } \\
74 \mathrm{~K} \text { taken } \sim 65 \mathrm{ft} \text { above base of section ( } 23 \mathrm{ft} \text { below top of Kuna). } \\
\text { 74L taken } \sim 73 \mathrm{ft} \text { above base of section ( } 15 \mathrm{ft} \text { below top of Kuna). }\end{array}$ \\
\hline & & $\begin{array}{l}\text { Scharfenbergia tailleurense Holdsworth and Murchey } \\
{[97 \mathrm{AK} 74 \mathrm{M} \text { and X] }}\end{array}$ & & $\begin{array}{l}\text { Samples from Kuna Formation. } \\
74 \mathrm{M} \text { taken } \sim 78 \mathrm{ft} \text { above base of section ( } 10 \mathrm{ft} \text { below top of Kuna) } \\
\text { from an interval of siliceous mudstone and } \leq 10 \% \text { medium-dark- } \\
\text { gray to black chert in beds } 0.5 \text { to } 3 \mathrm{~cm} \text { thick. } \\
74 \mathrm{X} \text { taken } \sim 83 \mathrm{ft} \text { above base of section ( } 5 \mathrm{ft} \text { below top of Kuna) from } \\
\text { hard vitreous chert in beds } 3 \text { to } 15 \mathrm{~cm} \text { thick that form mammiform } \\
\text { structures as much as } 1 \mathrm{ft} \text { high and } 2 \mathrm{ft} \text { across. } \\
\end{array}$ \\
\hline & & $\begin{array}{l}\text { ?Scharfenbergia ruestae (Ormiston and Lane) } \\
\text { Scharfenbergia tailleurense Holdsworth and Murchey } \\
\text { late scharfenbergiid" form of Holdsworth and Murchey } \\
\text { [97AK } 74 \mathrm{~N}]\end{array}$ & & $\begin{array}{l}\text { Sample at top of Kuna Formation, } \sim 88 \mathrm{ft} \text { above base of section and } \\
\text { just below contact with overlying Siksikpuk Formation. } \\
\text { Sample from interval of vitreous chert with mammiform structures. }\end{array}$ \\
\hline
\end{tabular}


Table 14. Radiolarian samples from the De Long Mountains quadrangle-Continued.

[All faunas identified by C.D. Blome unless otherwise indicated. Structural units determined by L.E. Young. EMA, Endicott Mountains allochthon; KRA, Kelly River allochthon; PCA, Picnic Creek allochthon. Letters in field number refer to collector: AD, J.A. Dumoulin; AK, J.S. Kelley; and JS, J.M. Schmidt. DDH, diamond drill hole. Interval thicknesses in drill holes are drilled thicknesses and have not been corrected for dip of bedding. All drill hole samples collected by J.A. Dumoulin and (or) A.G. Harris unless otherwise indicated. Lithologic data under remarks are field descriptions unless otherwise indicated; thin section observations by J.A. Dumoulin. No., number; loc., locality]

\begin{tabular}{|c|c|c|c|c|}
\hline $\begin{array}{l}\text { Locality No., } \\
\text { structural unit }\end{array}$ & $\begin{array}{l}\text { Quadrangle, } \\
\text { latitude } \mathrm{n} . / \\
\text { longitude w. }\end{array}$ & $\begin{array}{c}\text { Radiolarian fauna } \\
\text { [field No.; USGS collection No.] }\end{array}$ & Age & Remarks \\
\hline \multirow[t]{5}{*}{$\begin{array}{l}107 \\
\text { EMA (Key } \\
\text { Creek plate) } \\
\text { [Cont.] }\end{array}$} & \multirow[t]{5}{*}{$\begin{array}{l}\text { De Long Mtns. } \\
\text { B-1 } \\
68^{\circ} 15^{\prime} 31.4^{\prime \prime} \\
162^{\circ} 13^{\prime} 20^{\prime \prime}\end{array}$} & $\begin{array}{l}\text { ?Scharfenbergia ruestae (Ormiston and Lane) } \\
\text { Scharfenbergia tailleurense Holdsworth and Murchey } \\
{[97 \mathrm{AK} 74 \mathrm{O}]}\end{array}$ & \multirow[t]{3}{*}{$\begin{array}{l}\text { late Late Mississippian to } \\
\text { early Middle Pennsylvanian } \\
\text { (Chesterian to Morrowan). }\end{array}$} & $\begin{array}{l}\text { Sample from Siksikpuk Formation, } \sim 93 \mathrm{ft} \text { above base of section and } \\
5 \mathrm{ft} \text { above base of unit, from medium-dark-gray to black chert in } \\
\text { even to nodular beds } 3 \text { to } 10 \mathrm{~cm} \text { thick. }\end{array}$ \\
\hline & & $\begin{array}{l}\text { Scharfenbergia tailleurense Holdsworth and Murchey } \\
\text { [97AK74P] }\end{array}$ & & $\begin{array}{l}\text { Sample from Siksikpuk Formation, } \sim 102 \mathrm{ft} \text { above base of section and } \\
14 \mathrm{ft} \text { above base of unit, from medium-dark-gray to black chert in } \\
\text { even to nodular beds } 3 \text { to } 10 \mathrm{~cm} \text { thick. }\end{array}$ \\
\hline & & $\begin{array}{l}\text { Scharfenbergia tailleurense Holdsworth and Murchey } \\
\text { "late scharfenbergiid" form of Holdsworth and Murchey } \\
\text { [97AK74Q] }\end{array}$ & & $\begin{array}{l}\text { Sample from Siksikpuk Formation, } \sim 108 \mathrm{ft} \text { above base of section and } \\
20 \mathrm{ft} \text { above base of unit, from greenish-gray chert in beds } 1 \text { to } 10 \\
\mathrm{~cm} \text { thick. }\end{array}$ \\
\hline & & $\begin{array}{l}\text { Glassy spheres (probable casts of spumellarians) } \\
\text { [97AK74R and S] }\end{array}$ & $\begin{array}{l}\text { late Late Mississippian to } \\
\text { early Middle Pennsylvanian } \\
\text { (Chesterian to Morrowan), } \\
\text { based on age of underlying } \\
\text { and overlying samples. }\end{array}$ & $\begin{array}{l}\text { Samples from Siksikpuk Formation, medium-gray to greenish-gray } \\
\text { chert in beds } 1 \text { to } 5 \mathrm{~cm} \text { thick. } \\
74 \mathrm{R} \text { taken } \sim 113 \mathrm{ft} \text { above base of section ( } 25 \mathrm{ft} \text { above base of unit). } \\
74 \mathrm{~S} \text { taken } \sim 118 \mathrm{ft} \text { above base of section ( } 30 \mathrm{ft} \text { above base of unit). }\end{array}$ \\
\hline & & $\begin{array}{l}\text { Paroneaella? triporosa Holdsworth and Murchey } \\
\text { ?Scharfenbergia ruestae (Ormiston and Lane) } \\
\text { Scharfenbergia tailleurense Holdsworth and Murchey } \\
\text { "late scharfenbergiid" form of Holdsworth and Murchey } \\
\text { [97AK74T] }\end{array}$ & $\begin{array}{l}\text { Probably Early-early Middle } \\
\text { Pennsylvanian (Morrowan), } \\
\text { based on co-occurrence of } \\
\text { P.? triporosa and } S . \\
\text { tailleurense. } P . ? \text { triporosa } \\
\text { in Alaska occurs with } \\
\text { conodonts of early } \\
\text { Morrowan age, and may } \\
\text { range into the early Atokan, } \\
\text { but the range of } S \text {. } \\
\text { tailleurense is most likely } \\
\text { Chesterian to Morrowan. } \\
\end{array}$ & $\begin{array}{l}\text { Sample from Siksikpuk Formation, } \sim 153 \mathrm{ft} \text { above base of section and } \\
65 \mathrm{ft} \text { above base of unit, from interbedded grayish-green chert } \\
\text { (beds } 2 \text { to } 4 \mathrm{~cm} \text { ) and medium-grayish-green shale (beds } 0.5 \text { to } 2 \\
\mathrm{~cm} \text { ). }\end{array}$ \\
\hline 112 & $\begin{array}{l}\text { De Long Mtns. } \\
\text { A-1 } \\
68^{\circ} 11^{\prime} 44.8^{\prime \prime} \\
162^{\circ} 26^{\prime} 46.5^{\prime \prime}\end{array}$ & $\begin{array}{l}\text { Scharfenbergia tailleurense Holdsworth and Murchey } \\
\text { "late scharfenbergiid" form of Holdsworth and Murchey } \\
\text { [97AK40A] }\end{array}$ & $\begin{array}{l}\text { late Late Mississippian to } \\
\text { early Middle Pennsylvanian } \\
\text { (Chesterian to Morrowan) }\end{array}$ & $\begin{array}{l}\text { Kuna Formation, } \sim 20 \mathrm{ft} \text { below contact with overlying Etivluk Group. } \\
\text { Contact marked by horizon of mammilary forms }(?) \leq 0.5 \mathrm{~m} \text { across. } \\
\text { Dark-gray to black chert in blocky beds } 5 \text { to } 20 \mathrm{~cm} \text { thick, with } \\
\text { partings of black siliceous shale. }\end{array}$ \\
\hline $\begin{array}{l}126 \\
\text { EMA } \\
\text { (Wolverine } \\
\text { Creek plate) }\end{array}$ & $\begin{array}{l}\text { De Long Mtns. } \\
\text { A-2 } \\
68^{\circ} 09^{\prime} 20^{\prime \prime} \\
163^{\circ} 01^{\prime} 40^{\prime \prime}\end{array}$ & $\begin{array}{l}\text { Muelleritortis cochleata cochleata (Nakaseko and Nishimura) } \\
\text { Paronaella sp. aff. P. fragilis Kozur and Mostler } \\
\text { Pseudostylosphaera compacta (Nakaseko and Nishimura) } \\
\text { Pseudostylosphaera hellenica (DeWever) } \\
\text { Pseudostylosphaera japonica (Nakaseko and Nishimura) } \\
\text { Tritortis sp. } \\
\text { [DDH } 927 \text { (west of Anarraaq deposit), } 1752 \mathrm{ft} \text {; DR 2519] }\end{array}$ & $\begin{array}{l}\text { late Middle to early Late } \\
\text { Triassic (late Ladinian to } \\
\text { early Carnian) }\end{array}$ & $\begin{array}{l}\text { Sample from middle subunit of Otuk Formation (Etivluk Group), } 46 \\
\mathrm{ft} \text { below contact with upper subunit, } 20 \mathrm{ft} \text { below base of recessive } \\
\text { marker in middle subunit, and } 37 \mathrm{ft} \text { above contact with lower } \\
\text { subunit. } \\
\text { Light to medium gray chert, locally greenish gray. Thin section } \\
\text { shows moderately well preserved, abundant radiolarians, some } \\
\text { with sharp margins; scattered dolomite rhombs. }\end{array}$ \\
\hline
\end{tabular}


Table 14. Radiolarian samples from the De Long Mountains quadrangle-Continued.

[All faunas identified by C.D. Blome unless otherwise indicated. Structural units determined by L.E. Young. EMA, Endicott Mountains allochthon; KRA, Kelly River allochthon; PCA, Picnic Creek allochthon. Letters in field number refer to collector: AD, J.A. Dumoulin; AK, J.S. Kelley; and JS, J.M. Schmidt. DDH, diamond drill hole. Interval thicknesses in drill holes are drilled thicknesses and have not been corrected for dip of bedding. All drill hole samples collected by J.A. Dumoulin and (or) A.G. Harris unless otherwise indicated. Lithologic data under remarks are field descriptions unless otherwise indicated; thin section observations by J.A. Dumoulin. No., number; loc., locality]

\begin{tabular}{|c|c|c|c|c|}
\hline $\begin{array}{l}\text { Locality No., } \\
\text { structural unit }\end{array}$ & $\begin{array}{l}\text { Quadrangle, } \\
\text { latitude n./ } \\
\text { longitude w. }\end{array}$ & $\begin{array}{c}\text { Radiolarian fauna } \\
\text { [field No.; USGS collection No.] }\end{array}$ & Age & Remarks \\
\hline $\begin{array}{l}131 \\
\text { EMA (Red Dog } \\
\text { plate) }\end{array}$ & $\begin{array}{l}\text { De Long Mtns. } \\
\text { A-2 } \\
68^{\circ} 09^{\prime} 46^{\prime \prime} \\
162^{\circ} 57^{\prime} 44^{\prime \prime}\end{array}$ & $\begin{array}{l}\text { Scharfenbergia sp. aff. S. tailleurense Holdsworth and Murchey } \\
\text { All radiolarians poorly preserved as casts only } \\
\text { [DDH } 813 \text { (Anarraaq deposit), } 1771 \mathrm{ft} \text {; DR 2518] }\end{array}$ & Paleozoic & $\begin{array}{l}\text { Ikalukrok unit, } 8 \mathrm{ft} \text { below contact with Siksikpuk Formation and } 21 \mathrm{f} \\
\text { above base of chert interval at top of unit. See table } 1 \text { for conodont } \\
\text { samples from this drill hole. } \\
\text { Dark-gray chert. Thin section shows moderate to abundant } \\
\text { radiolarians filled with fine-crystalline quartz or chalcedony in } \\
\text { dark muddy matrix; margins sharp and rim structure locally well } \\
\text { preserved. }\end{array}$ \\
\hline \multirow[t]{2}{*}{$\begin{array}{l}136 \\
\text { EMA (Red Dog } \\
\text { plate) }\end{array}$} & \multirow[t]{2}{*}{$\begin{array}{l}\text { De Long Mtns. } \\
\text { A-2 } \\
68^{\circ} 06^{\prime} 14.4^{\prime \prime} / \\
162^{\circ} 50^{\prime} 29.8^{\prime \prime}\end{array}$} & $\begin{array}{l}\text { Albaillella sp. aff. A. furcata group Won ( } 2 \text { forms) } \\
\text { All forms very poorly preserved } \\
\text { [DDH } 50 \text { (north of Paalaaq deposit), } 215 \mathrm{ft} \text {; DR 2457] }\end{array}$ & $\begin{array}{l}\text { Late Mississippian-early } \\
\text { Middle Pennsylvanian } \\
\text { (Meramecian to } \\
\text { Morrowan). }\end{array}$ & $\begin{array}{l}\text { Siksikpuk Formation, just above contact with black chert of the } \\
\text { Ikalukrok unit (Kuna Formation). The contact between the two } \\
\text { units may be faulted; breccia occurs along it. } \\
\text { Medium-light-gray (to light bluish gray) chert. Radiolarians notable } \\
\text { in hand sample, but thin section shows only a few round } \\
\text { radiolarian ghosts (some filled with barite). } \\
\text { Loc. } 23 \text { (fig. 2) of Dumoulin and others (2004). }\end{array}$ \\
\hline & & $\begin{array}{l}\text { Albaillella } \text { sp. aff. A. cartalla Ormiston and Lane } \\
\text { Paleoxyphostylus variospina Won } \\
\text { Scharfenbergia tailleurense Holdsworth and Murchey } \\
\text { Triactofenestrella sp. } \\
\text { [DDH 50, } 219 \mathrm{ft} \text {; DR 2489] }\end{array}$ & \begin{tabular}{|l} 
late Late Mississippian-early \\
Middle Pennsylvanian \\
(Chesterian to Morrowan). \\
The concurrent ranges of \\
these four species indicate \\
that the fauna is assignable \\
to the Scharfenbergia \\
tailleurense assemblage \\
(Assemblage Zone 5) of \\
Murchey (1990). \\
\end{tabular} & $\begin{array}{l}\text { Black chert of the Ikalukrok unit (Kuna Formation), about } 4 \mathrm{ft} \text { below } \\
\text { contact with Siksikpuk Formation. } \\
\text { Grayish-black to black chert with notable radiolarians. Thin section } \\
\text { shows abundant, locally well preserved radiolarians (sharp edges) } \\
\text { in a muddy matrix; some tests filled with pyrite, others with barite, } \\
\text { but most with chalcedony. }\end{array}$ \\
\hline $\begin{array}{l}150 \\
\text { EMA (Red Dog } \\
\text { plate) }\end{array}$ & $\begin{array}{l}\text { De Long Mtns. } \\
\text { A-2 } \\
68^{\circ} 04^{\prime} 23^{\prime \prime} / \\
162^{\circ} 50^{\prime} 3.7^{\prime \prime}\end{array}$ & $\begin{array}{l}\text { Albaillella sp. aff. A. furcata group Won } \\
\text { ?Scharfenbergia sp. (one poorly preserved arm) } \\
\text { All radiolarians preserved as casts } \\
\text { [DDH } 484 \text { (Main deposit), } 208 \mathrm{ft} \text {; DR 2458] }\end{array}$ & \begin{tabular}{r|} 
?Mississippian (questionable \\
Meramecian to Morrowan)
\end{tabular} & $\begin{array}{l}\text { Shale and chert of Siksikpuk Formation; sample is about } 12 \mathrm{ft} \text { above } \\
\text { an interval of pyritic barite that is } \sim 8.5 \mathrm{ft} \text { thick and appears to be in } \\
\text { gradational contact with the chert. } \\
\text { Grayish-blue radiolarian chert interbedded with green mudstone; } \\
\text { sample from chert interval } \sim 1 \text { inch thick. } \\
\text { Sample collected by K.D. Kelley. }\end{array}$ \\
\hline
\end{tabular}


Table 14. Radiolarian samples from the De Long Mountains quadrangle-Continued.

[All faunas identified by C.D. Blome unless otherwise indicated. Structural units determined by L.E. Young. EMA, Endicott Mountains allochthon; KRA, Kelly River allochthon; PCA, Picnic Creek allochthon. Letters in field number refer to collector: AD, J.A. Dumoulin; AK, J.S. Kelley; and JS, J.M. Schmidt. DDH, diamond drill hole. Interval thicknesses in drill holes are drilled thicknesses and have not been corrected for dip of bedding. All drill hole samples collected by J.A. Dumoulin and (or) A.G. Harris unless

\begin{tabular}{|c|c|c|c|c|}
\hline $\begin{array}{l}\text { Locality No., } \\
\text { structural unit }\end{array}$ & $\begin{array}{l}\text { Quadrangle, } \\
\text { latitude n./ } \\
\text { longitude w. }\end{array}$ & $\begin{array}{c}\text { Radiolarian fauna } \\
\text { [field No.; USGS collection No.] }\end{array}$ & Age & Remarks \\
\hline $\begin{array}{l}151 \\
\text { EMA (Red Dog } \\
\text { plate) }\end{array}$ & $\begin{array}{l}\text { De Long Mtns. } \\
\text { A-2 } \\
68^{\circ} 04^{\prime} 21.2^{\prime \prime \prime} \\
162^{\circ} 49^{\prime} 59^{\prime \prime}\end{array}$ & $\begin{array}{l}\text { Small poorly preserved radiolarian, probably internal spicules } \\
\text { Few casts of ?Albaillella sp. } \\
\text { All forms very poorly preserved } \\
\text { [DDH } 24 \text { (Main deposit), } 126 \mathrm{ft} \text {; DR 2455] }\end{array}$ & ?Paleozoic & $\begin{array}{l}\text { Ikalukrok unit (Kuna Formation). } \\
\text { Black chert with numerous fractures filled with silica and lesser } \\
\text { pyrite. } \\
\text { Sample collected by K.D. Kelley. }\end{array}$ \\
\hline $\begin{array}{l}152 \\
\text { EMA (Red Dog } \\
\text { plate) }\end{array}$ & $\begin{array}{l}\text { De Long Mtns. } \\
\text { A-2 } \\
68^{\circ} 04^{\prime} 17.5^{\prime \prime} / \\
162^{\circ} 49^{\prime} 47.8^{\prime \prime}\end{array}$ & $\begin{array}{l}\text { Capnuchosphaera sp. (isolated spines called "tumidaspines") } \\
\text { Corum sp. aff. C. perfectum Blome } \\
\text { Corum sp. } \\
\text { Triassocampe sp. } \\
\text { All radiolarians poorly preserved and broken } \\
\text { [DDH } 42 \text { (Main deposit), } 325 \mathrm{ft} \text {; DR 2456] }\end{array}$ & $\begin{array}{l}\text { Late Triassic (late Carnian to } \\
\text { middle Norian). Fauna } \\
\text { assignable to the } \\
\text { Capnodoce Zone of Blome } \\
\text { (1984) and Blome and } \\
\text { others (1988). }\end{array}$ & $\begin{array}{l}\text { Sample from } \sim 10 \text {-ft-thick section of chert and shale logged as } \\
\text { Siksikpuk Formation; collected } \sim 1 \mathrm{ft} \text { above } \sim 15 \text {-ft-thick barite } \\
\text { interval. Radiolarian fauna indicates that unit sampled is the Otuk } \\
\text { Formation. } \\
\text { Dark-grey radiolarian chert interbedded with greenish shale. Chert } \\
\text { interval sampled cut by many silica-filled veins, including one } \\
\text { almost } 0.5 \text { inch wide. } \\
\text { Sample collected by K.D. Kelley. }\end{array}$ \\
\hline
\end{tabular}


Table 15. Conodonts illustrated in Plates 3 and 4 from the Howard Pass quadrangle

[All faunas identified by A.G. Harris. Structural units from Dumoulin and others (2004). CAI, conodont color alteration index. Letters in field number refer to collector: AD, J.A. Dumoulin; ADo, J.H. Dover, ARm, R.T. Miyaoka, and Tr, I.L.Tailleur. * species assignment revised after original publication. No., number; loc., locality]

\begin{tabular}{|c|c|c|c|c|c|}
\hline $\begin{array}{c}\text { Field and } \\
\text { USGS } \\
\text { collection Nos. }\end{array}$ & $\begin{array}{l}\text { Quadrangle, } \\
\text { latitude n./ } \\
\text { longitude w. }\end{array}$ & $\begin{array}{l}\text { Stratigraphic and } \\
\text { structural units }\end{array}$ & Age and CAI of collection & Conodont illustrated & Reference, remarks \\
\hline $\begin{array}{l}\text { 92AD35AA } \\
\text { 32441-PC }\end{array}$ & $\begin{array}{l}\text { Howard Pass } \\
\text { B-3 } \\
68^{\circ} 19^{\prime} 55^{\prime \prime} / \\
157^{\circ} 44^{\prime} 10^{\prime \prime}\end{array}$ & $\begin{array}{l}\text { Kuna Formation } \\
\text { (Lisburne Group) } \\
\text { Endicott Mountains } \\
\text { allochthon (Key } \\
\text { Creek plate) }\end{array}$ & $\begin{array}{l}\text { late Early Mississippian; Upper G. typicus } \\
\text { Subzone or lower part Sc. anchoralis-Do. } \\
\text { latus Zone (early middle Osagean) on the } \\
\text { basis of age constraints from overlying } \\
\text { sample. } \\
\text { CAI= 3 }\end{array}$ & $\begin{array}{l}\text { Bispathodus utahensis Sandberg and } \\
\text { Gutschick [pl. 3, figs. 17-20] } \\
\text { *Gnathodus cuneiformis Mehl and Thomas } \\
\text { [pl. 3, fig. 21] }\end{array}$ & $\begin{array}{l}\text { Dover and others (2004), table 1, loc. } 147 . \\
\text { Collected at type locality of Kuna Formation (as } \\
\text { designated in Mull and others, } 1982 \text { ) from } \\
\text { small outcrop at stream level } \sim 15 \text { m west and } \\
12 \mathrm{~m} \text { topographically lower than base of } \\
\text { 92AD35A measured section. If } 92 \mathrm{AD} 35 \mathrm{AA} \\
\text { strata are in place, they are the lowest } \\
\text { exposure at the type locality. }\end{array}$ \\
\hline $\begin{array}{l}\text { 92AD35C- } \\
15.5 \mathrm{~m} \\
32442-\mathrm{PC}\end{array}$ & $\begin{array}{l}\text { Howard Pass } \\
\text { B-3 } \\
68^{\circ} 20^{\prime} 00^{\prime \prime} / \\
157^{\circ} 44^{\prime} 35^{\prime \prime}\end{array}$ & $\begin{array}{l}\text { Kuna Formation } \\
\text { (Lisburne Group) } \\
\text { Endicott Mountains } \\
\text { allochthon (Key } \\
\text { Creek plate) }\end{array}$ & $\begin{array}{l}\text { late Early Mississippian; Sc. anchoralis-Do. } \\
\text { latus Zone (middle Osagean) } \\
\text { CAI }=\sim 3\end{array}$ & $\begin{array}{l}\text { Scaliognathus anchoralis Branson and } \\
\text { Mehl [pl. 3, fig. 25] }\end{array}$ & $\begin{array}{l}\text { Dover and others (2004), table 1, loc. } 146 . \\
\text { Collected from } 15.5 \mathrm{~m} \text { above base of } \sim 68 \mathrm{~m}- \\
\text { thick section of Kuna Formation at type } \\
\text { locality (as designated in Mull and others, } \\
\text { 1982). }\end{array}$ \\
\hline $\begin{array}{l}\text { 92AD55-22 } \\
\text { 32450-PC }\end{array}$ & $\begin{array}{l}\text { Howard Pass } \\
\text { B-3 } \\
68^{\circ} 19^{\prime} 36^{\prime \prime} / \\
157^{\circ} 45^{\prime} 15^{\prime \prime}\end{array}$ & $\begin{array}{l}\text { Kayak Shale } \\
\text { (Endicott Group) } \\
\text { Endicott Mountains } \\
\text { allochthon (Key } \\
\text { Creek plate) }\end{array}$ & $\begin{array}{l}\text { early Early Mississippian (Kinderhookian) } \\
\mathrm{CAI}=4\end{array}$ & $\begin{array}{l}\text { Bispathodus aculeatus plumulus Rhodes, } \\
\text { Austin, and Druce [pl. 3, figs. 11-12] } \\
\text { *Pseudopolygnathus nodomarginatus } \\
\text { (E.R. Branson) [pl. 3, figs. 13-14] }\end{array}$ & $\begin{array}{l}\text { Dover and others (2004), table } 4 \text {, loc. } 148, \\
\text { Dumoulin and Harris (1997), table } 1, \text { loc. } 2 . \\
\text { About } 1 \mathrm{~km} \mathrm{SW} \text { of type section of the Kuna } \\
\text { Formation; limestone and shale member of the } \\
\text { Kayak Shale, about } 12 \mathrm{~m} \text { below contact with } \\
\text { Rough Mountain Creek unit. }\end{array}$ \\
\hline $\begin{array}{l}\text { 91ADo70C } \\
\text { 31748-PC }\end{array}$ & $\begin{array}{l}\text { Howard Pass } \\
\text { B-3 } \\
68^{\circ} 22^{\prime} 52^{\prime \prime} / \\
157^{\circ} 40^{\prime} 50^{\prime \prime}\end{array}$ & $\begin{array}{l}\text { Kayak Shale } \\
\text { (Endicott Group) } \\
\text { Endicott Mountains } \\
\text { allochthon (Key } \\
\text { Creek plate) } \\
\end{array}$ & $\begin{array}{l}\text { early Early Mississippian (middle to late } \\
\text { Kinderhookian) } \\
\text { CAI= 3 }\end{array}$ & $\begin{array}{l}\text { Bispathodus aculeatus plumulus Rhodes, } \\
\text { Austin, and Druce, nodosus morphotype } \\
\text { [pl. 3, fig. 16] }\end{array}$ & $\begin{array}{l}\text { Dover and others (2004), table 4, loc. 143, } \\
\text { Dumoulin and Harris (1997), table 1, loc. 6, } \\
\text { and Mull and others (1997), table 1. } \\
\text { Limestone and shale member of the Kayak Shale } \\
\text { (Mull and others, 1997). }\end{array}$ \\
\hline $\begin{array}{l}\text { 91AD6U } \\
\text { 31742-PC }\end{array}$ & $\begin{array}{l}\text { Howard Pass } \\
\text { C-3 } \\
68^{\circ} 32^{\prime} 00^{\prime \prime} / \\
157^{\circ} 34^{\prime} 55^{\prime \prime}\end{array}$ & $\begin{array}{l}\text { Rim Butte unit } \\
\text { (Lisburne Group) } \\
\text { Ipnavik River } \\
\text { allochthon } \\
\text { (Ipnavik plate) }\end{array}$ & $\begin{array}{l}\text { No older than late Early Mississippian (no older } \\
\text { than middle Osagean). } \\
{[1.5-2]}\end{array}$ & $\begin{array}{l}\text { Bispathodus utahensis Sandberg and } \\
\text { Gutschick [pl. 3, fig. 15] }\end{array}$ & $\begin{array}{l}\text { Dover and others (2004), table 4, loc. } 54, \\
\text { Dumoulin and others (1993), table 1, loc. } 21 . \\
\text { From } 67 \text {-meter-thick measured section within } \\
\text { Rim Butte unit, } 61 \text { m above base of section. }\end{array}$ \\
\hline $\begin{array}{l}\text { 92ARm37B } \\
\text { 32471-PC }\end{array}$ & $\begin{array}{l}\text { Howard Pass } \\
\text { B-5 } \\
68^{\circ} 233^{\prime} 30^{\prime \prime} / \\
158^{\circ} 48^{\prime} 55^{\prime \prime}\end{array}$ & $\begin{array}{l}\text { Rim Butte unit } \\
\text { (Lisburne Group) } \\
\text { Ipnavik River } \\
\text { allochthon } \\
\text { (Ipnavik plate) } \\
\end{array}$ & $\begin{array}{l}\text { late Early Mississippian; Sc. anchoralis-Do. } \\
\text { latus Zone (middle Osagean). } \\
\text { CAI=3 }\end{array}$ & $\begin{array}{l}\text { Geniculatus claviger Hass, s.f. [pl. 3, figs. } \\
\text { 22-23] }\end{array}$ & Dover and others (2004), table 1, loc. 91. \\
\hline $\begin{array}{l}91 \mathrm{Tr} 09 \mathrm{~B} \\
31840-\mathrm{PC}\end{array}$ & $\begin{array}{l}\text { Howard Pass } \\
\text { C-3 } \\
68^{\circ} 31.07^{\prime} / \\
157^{\circ} 36.05^{\prime}\end{array}$ & $\begin{array}{l}\text { Rim Butte unit } \\
\text { (Lisburne Group) } \\
\text { Ipnavik River } \\
\text { allochthon } \\
\text { (Ipnavik plate) }\end{array}$ & $\begin{array}{l}\text { late Early Mississippian; lower half } S c \text {. } \\
\text { anchoralis-Do. latus Zone (middle Osagean). } \\
\text { CAI=1.5-2 }\end{array}$ & $\begin{array}{l}\text { Doliognathus latus Branson and Mehl [pl. } \\
4 \text {, figs. } 3,4] \\
* \text { Geniculatus claviger Hass, s.f. [pl. 3, fig. } \\
24]\end{array}$ & Dover and others (2004), table 1, loc. 60. \\
\hline
\end{tabular}


Table 15. Conodonts illustrated in Plates 3 and 4 from the Howard Pass quadrangle

[All faunas identified by A.G. Harris. Structural units from Dumoulin and others (2004). CAI, conodont color alteration index. Letters in field number refer to collector: AD, J.A. Dumoulin; ADo, J.H. Dover, ARm, R.T. Miyaoka, and Tr, I.L.Tailleur. * species assignment revised after original publication. No., number; loc., locality]

\begin{tabular}{|c|c|c|c|c|c|}
\hline $\begin{array}{l}\text { Field and } \\
\text { USGS } \\
\text { collection Nos. }\end{array}$ & $\begin{array}{l}\text { Quadrangle, } \\
\text { latitude n./ } \\
\text { longitude w. }\end{array}$ & $\begin{array}{l}\text { Stratigraphic and } \\
\text { structural units }\end{array}$ & Age and CAI of collection & Conodont illustrated & Reference, remarks \\
\hline $\begin{array}{l}91 \mathrm{Tr} 13 \\
31841-\mathrm{PC}\end{array}$ & $\begin{array}{l}\text { Howard Pass } \\
\text { C-3 } \\
68^{\circ} 31.1^{\prime \prime} / \\
157^{\circ} 35.5^{\prime}\end{array}$ & $\begin{array}{l}\text { Rim Butte unit } \\
\text { (Lisburne Group) } \\
\text { Ipnavik River } \\
\text { allochthon } \\
\text { (Ipnavik plate) }\end{array}$ & $\begin{array}{l}\text { late Early Mississippian; lower half } S c \text {. } \\
\text { anchoralis-Do. latus Zone (middle Osagean). } \\
\mathrm{CAI}=\sim 2+\text { heavy gray patina }\end{array}$ & $\begin{array}{l}\text { *Gnathodus pseudosemiglaber Thompson } \\
\text { and Fellows [pl. 3, figs. 28, 30] } \\
\text { Protognathodus cordiformis Lane, } \\
\text { Sandberg and Ziegler [pl. 3, fig. 26] } \\
\text { *Pseudopolygnathus marginata (Branson } \\
\text { and Mehl) [pl. 3, fig. 27] }\end{array}$ & Dover and others (2004), table 1, loc. 59. \\
\hline $\begin{array}{l}\text { 92AD20-14.5 } \\
32426-\mathrm{PC}\end{array}$ & $\begin{array}{l}\text { Howard Pass } \\
\text { C-3 } \\
68^{\circ} 38^{\prime} 10^{\prime \prime} / \\
157^{\circ} 31^{\prime} 40^{\prime \prime}\end{array}$ & $\begin{array}{l}\text { Rim Butte unit } \\
\text { (Lisburne Group) } \\
\text { Ipnavik River } \\
\text { allochthon } \\
\text { (Ipnavik plate) }\end{array}$ & $\begin{array}{l}\text { No older than late Early Mississippian; } S c \text {. } \\
\text { anchoralis-Do. latus Zone (middle Osagean or } \\
\text { younger) with redeposited Famennian (late } \\
\text { Late Devonian) and middle-late } \\
\text { Kinderhookian conodonts. } \\
\text { CAI }=1.5\end{array}$ & $\begin{array}{l}\text { Palmatolepis sp. of Famennian } \\
\text { morphotype redeposited during middle } \\
\text { Osagean or younger Mississippian time } \\
\text { [pl. 3, fig. 29] }\end{array}$ & $\begin{array}{l}\text { Dover and others ( } 2004) \text {, table } 1 \text {, loc. } 27 . \\
\text { Collected about } 14.5 \mathrm{~m} \text { below top of } 42 \text {-m-thick } \\
\text { section of Rim Butte unit. }\end{array}$ \\
\hline $\begin{array}{l}\text { 92AD214-18.5 } \\
\text { 32464-PC }\end{array}$ & $\begin{array}{l}\text { Howard Pass } \\
\text { B-5 } \\
68^{\circ} 23^{\prime} 10^{\prime \prime} / \\
158^{\circ} 54^{\prime} 00^{\prime \prime}\end{array}$ & $\begin{array}{l}\text { Rim Butte unit } \\
\text { (Lisburne Group) } \\
\text { Ipnavik River } \\
\text { allochthon } \\
\text { (Ipnavik plate) }\end{array}$ & $\begin{array}{l}\text { late Early Mississippian; Sc. anchoralis-Do. } \\
\text { latus Zone (middle Osagean). } \\
\text { CAI=2.5 }\end{array}$ & $\begin{array}{l}\text { Doliognathus latus Branson and Mehl [pl. } \\
\text { 4, figs. } 1,2]\end{array}$ & $\begin{array}{l}\text { Dover and others (2004), table } 1, \text { loc. } 89 \text {. } \\
\text { Collected from } 18.5 \mathrm{~m} \text { above base of } \sim 85-\mathrm{m}- \\
\text { thick measured section of Rim Butte unit. }\end{array}$ \\
\hline $\begin{array}{l}\text { 92AD214-44 } \\
\text { 32465-PC }\end{array}$ & $\begin{array}{l}\text { Howard Pass } \\
\text { B-5 } \\
68^{\circ} 23^{\prime} 10^{\prime \prime} / \\
158^{\circ} 54^{\prime} 00^{\prime \prime}\end{array}$ & $\begin{array}{l}\text { Rim Butte unit } \\
\text { (Lisburne Group) } \\
\text { Ipnavik River } \\
\text { allochthon } \\
\text { (Ipnavik plate) } \\
\end{array}$ & $\begin{array}{l}\text { late Early Mississippian Sc. anchoralis-Do. } \\
\text { latus Zone (middle Osagean). } \\
\text { CAI }=2.5-3\end{array}$ & $\begin{array}{l}\text { Mestognathus praebeckmanni Sandberg, } \\
\text { Johnson, Orchard, and von Bitter [pl. 4, } \\
\text { figs. 5, 6] }\end{array}$ & $\begin{array}{l}\text { Dover and others (2004), table } 1, \text { loc. } 89 \text {. } \\
\text { Collected from } 44 \text { m above base of } \sim 85 \text {-m-thick } \\
\text { measured section of Rim Butte unit. }\end{array}$ \\
\hline $\begin{array}{l}\text { 92AD50C } \\
32447-\mathrm{PC}\end{array}$ & $\begin{array}{l}\text { Howard Pass } \\
\text { C-5 } \\
68^{\circ} 44^{\prime} 45^{\prime \prime} / \\
158^{\circ} 55^{\prime} 35^{\prime \prime}\end{array}$ & $\begin{array}{l}\text { Rim Butte unit } \\
\text { (Lisburne Group) } \\
\text { Ipnavik River } \\
\text { allochthon } \\
\text { (Ipnavik plate) }\end{array}$ & $\begin{array}{l}\text { No older than late Early Mississippian;Sc. } \\
\text { anchoralis-Do. latus Zone (middle Osagean) } \\
\text { with redeposited Kinderhookian and possibly } \\
\text { early Osagean conodonts } \\
\text { CAI=1.5-2 }\end{array}$ & $\begin{array}{l}\text { Gnathodus punctatus (Cooper), late } \\
\text { Kinderhookian-very early Osagean } \\
\text { species redeposited during middle } \\
\text { Osagean or younger Mississippian time } \\
\text { [pl. 4, fig. 7] }\end{array}$ & $\begin{array}{l}\text { Dover and others (2004), table } 1 \text {, loc. } 2 . \\
\text { Collected } \sim 15 \mathrm{~m} \text { below top of } \sim 50 \text { - to } 60 \text { m-thick } \\
\text { section of Rim Butte unit. }\end{array}$ \\
\hline
\end{tabular}




\section{PLATE 1}

Conodonts from the Kuna Formation and related deep-water facies of the Lisburne Group (scanning electron micrographs; illustrated specimens are reposited in the U.S. National

Museum, USNM, Washington, D.C.; see tables 1-4 and maps 1 and 2 for conodont fauna, age, biofacies, geologic setting, and location of collections listed below)

Kuna Formation, Ikalukrok unit (Red Dog plate, Endicott Mountains allochthon)

Figure 1. Gnathodus cuneiformis Mehl and Thomas, upper view of incomplete juvenile Pa element, X100, DDH Su31, 1667-1686 ft., USGS colln. 29280-PC, USNM No. 530472 (map 2, loc. 123).

2-4. Gnathodus cuneiformis Mehl and Thomas, Protognathodus praedelicatus Lane, Sandberg, and Ziegler, and Bispathodus utahensis Sandberg and Gutschick, 2 upper and 1 outer lateral views of Pa elements, X100, DH 807, 1282-1297 ft., Gul subplate of Red Dog plate, USGS colln. 33493-PC, USNM Nos. 530473-75 (map 2, loc. 128). 5, 6. Gnathodus texanus Roundy and Cavusgnathus unicornis Youngquist and Miller, upper views of Pa elements, X100, field no. 99AD11BB, USGS colln. 33479-PC, USNM Nos. 530476, 77 (map 1, loc. 76).

7. Gnathodus texanus Roundy, Pa element, upper view, X100, DDH Su16, 558-77 ft., USGS colln. 29279-PC, USNM No. 530478 (map 2, loc. 124).

8. Gnathodus sp., upper view of juvenile Pa element, X100, field no. KE98-19B, USGS colln. 33644-PC, USNM No. 530479 (map 1, no. 48).

Kuna Formation, Kivalina unit (Red Dog plate, Endicott Mountains allochthon) 
9, 10. Scaliognathus anchoralis Branson and Mehl, inner lateral view of incomplete Sa element and lateral view of S element bar fragment, X100, DDH 640, 429-453 ft., Paalaaq deposit, USGS colln. 33488-PC, USNM Nos. 530480, 81 (map 2, no. 142).

Kuna Formation (Key Creek plate, Endicott Mountains allochthon)

11-13. Eotaphrus burlingtonensis Pierce and Langenheim, adult and juvenile Pa elements, upper and lower views, X100, field no. 79MD44C, USGS colln. 27506-PC, USNM Nos. 530482, 83 (map 1, no. 103).

14, 15. Polygnathus communis Branson and Mehl, Pa element, upper view, X180 and Scaliognathus anchoralis, Sc element, inner lateral view, X150, field no. 00AD17H, USGS colln. 33631-PC, USNM Nos. 530484, 85 (map 1, no. 103).

16, 17. Rhachistognathus prolixus Baesemann and Lane and Gnathodus texanus Roundy, Pa elements, upper view, X100, field no. 97AK74F, USGS colln. 33404-PC, USNM Nos. 530486, 87 (map 1, no. 107).

18, 19. Gnathodus cuneiformis Mehl and Thomas, juvenile and subadult Pa elements, upper view, X100, field no. 79MD44C, USGS colln. 27506-PC, USNM Nos. 530488, 89 (map 1, no. 103).

Deep-water facies of the Lisburne Group (Picnic Creek allochthon)

20. Cavusgnathus regularis Youngquist and Miller, juvenile Pa element, outer lateral view, X100, field no. KE98-24 (+590 ft.), Wulik plate, USGS colln. 33602-PC, USNM No. 530490 (map 1, no. 34).

21, 22. Bispathodus utahensis Sandberg and Gutschick, outer and inner lateral views of Pa elements, X100, field no. KE98-24 (+308 ft.), Wulik plate, USGS colln. 33600PC, USNM Nos. 530491, 92 (map 1, no. 34). 
23-25. Rhachistognathus prolixus Baesemann and Lane, Gnathodus cuneiformis Mehl and Thomas, and Gnathodus texanus Roundy, X100, field no. 99AD19D, Wulik plate, USGS colln. 33481-PC, USNM Nos. 530493-95 (map 1, no. 35).

26. Cavusgnathus regularis Youngquist and Miller, Pa element, upper view, X80, field no. 8-17-83B, Amaruk plate, USGS colln. 29236-PC, USNM No. 530496 (map 1, no. 20).

\section{PLATE 2}

Conodonts from deep-water facies of the Lisburne Group and from shallow-water facies (Kogruk and Utukok Formations) of the Lisburne Group (scanning electron micrographs; illustrated specimens are reposited in the U.S. National Museum, USNM, Washington, D.C.; see tables 4-7 and maps 1 and 4 for conodont fauna, age, biofacies, geologic setting, and location of collections listed below)

Deep-water facies of Lisburne Group (Picnic Creek allochthon) (continued)

Figures 1, 2. Hindeodus crassidentatus (Branson and Mehl), inner lateral and posterior views of Sc and Sa elements, X100, field no. KE98-24 (+382 ft.), Wulik plate, USGS colln. 33601-PC, USNM Nos. 530497, 98 (map 1, no. 34).

3, 4. Vogelgnathus cf. V. pesaquidi Purnell and von Bitter, outer lateral views of juvenile Pa elements, X150, field no. KE98-24 (+360 ft.), Wulik plate, USGS colln. 33643PC, USNM Nos. 530499, 530500 (map 1, no. 34).

5, 6. Scaliognathus anchoralis Branson and Mehl, lateral views of S element bar fragments, X100, field no. 00AD25F, Wulik plate, USGS colln. 33637-PC, USNM Nos. 530501, 02 (map 1, no.117). 
7-10. Eotaphrus burlingtonensis Pierce and Langenheim and Polygnathus communis Branson and Mehl, upper and lower views of Pa elements, X100, field no. 79EK184B, Wulik plate, USGS colln. 27554-PC, USNM No. 530503, 04 (map 1, no. 37).

Other deep-water strata of the Lisburne Group

11, 12. Geniculatus sp., upper and lower views of incomplete P element, X100, field no. 00AD26A, Nachralik Pass plate, Ipnavik River allochthon (IRA), USGS colln. 33638-PC, USNM 530505 (map 1, no. 120).

Kogruk Formation

13. Gnathodus homopunctatus Ziegler?, upper view, juvenile Pa element, X100, field no. 97AK51A, Kelly River allochthon (KRA), USGS colln. 33395-PC, USNM No. 530506 (map 1, no. 114).

14, 15. Polygnathus mehli Thompson, upper and lower views, Pa element, X100, field no. KE98-25B, Wolverine Creek plate in Rok window, Endicott Mountains allochthon, EMA, USGS colln. 33604-PC, USNM No. 530507 (map 1, no. 73).

Utukok Formation

16. Gnathodus cuneiformis Mehl and Thomas, upper view, juvenile Pa element, X100, field no. 00AD15A, Utukok Formation, Kelly plate, KRA, USGS colln. 33630-PC, USNM No. 530508 (map 1, no. 102).

17. Pseudopolygnathus sp., abraded subadult Pa element, upper view, X150, field no. 00AD6A, lower part of Utukok Formation, Key Creek plate, EMA, USGS colln. 33627-PC, USNM No. 530509 (map 1, no. 31). 
18, 19. Siphonodella sp., juvenile Pa element, lower and upper views, X150, field no. 81EK69C, Eli plate, KRA, USGS colln. 28583-PC, USNM No. 530510 (map 4, no. 167).

20-26. Polygnathus bischoffi Rhodes, Austin, and Druce, Pa element, upper and lower views, X100; Synclydognathus geminus (Hinde), Sb element, postero-lateral view, X100; Pseudopolygnathus marginatus (Branson and Mehl)?, upper and lower views of subadult Pa element, X150; and Pseudognathodus multistriatus Mehl and Thomas (figs. 25 and 26), X100, field no. 79EK175C; olistolith probably derived from Picnic Creek allochthon (PCA) or KRA, USGS colln. 27553-PC, USNM Nos. 530511-14 (map 1, no. 25).

27. Hindeodus crassidentatus (Branson and Mehl), outer lateral view of Pa element, X90, field no. KE98-22, Wolverine Creek plate in Rok window, EMA, USGS colln. 33506-PC, USNM No. 530515 (map 1, no. 63).

28, 29. Hindeodus crassidentatus (Branson and Mehl) and Synclydognathus geminus (Hinde), outer lateral views of Pa elements, X100, field no. 79CX119A,Wolverine Creek plate in Rok window, EMA, USGS colln. 33348-PC, USNM Nos. 530516, 17 (map 1, no. 64).

$$
\text { PLATE } 3
$$

Conodonts from various units of the Lisburne Group and from the Kayak Shale of the Endicott Group (scanning electron micrographs; illustrated specimens are reposited in the U.S. National Museum, USNM, Washington, D.C.; see tables 7, 11, 13, and 15 and maps 1 and 4 for conodont fauna, age, biofacies, geologic setting, and location of collections listed below) 
Utukok Formation (continued)

Figures 1, 2. Pseudopolygnathus sp. and Bispathodus utahensis Sandberg and Gutschick, upper view of juvenile and adult Pa elements, X150 and X100, respectively, field no. 97AK107A, Wulik Peaks plate, KRA, USGS colln. 33400-PC, USNM Nos. 530518, 19 (map 1, no. 52).

Kayak Shale, Endicott Group

3, 4. Polygnathus communis Branson and Mehl, upper and lower views of $\mathrm{Pa}$ element, X100, field no. 81EK75F, Key Creek plate, EMA, USGS colln. 28584-PC, USNM No. 530520 (map 4, no. 175).

5, 6. Synclydognathus geminus (Hinde), inner lateral views of incomplete $\mathrm{Pb}$ and $\mathrm{Sa}$ elements, X100, field no. 81MD41E, EMA, USGS colln. 28367-PC, USNM Nos. 530521, 22 (map 4, no. 178).

7, 8. Patrognathus variabilis Rhodes, Austin, and Druce, upper and inner lateral views of Pa element, X100, field no. 81EK75F, Key Creek plate, EMA, USGS colln. 28584PC, USNM No. 530523 (map 4, no. 175).

9, 10. Bispathodus stabilis (Branson and Mehl), outer and inner lateral views, Pa and Sc elements, X100, field no. 81EK100A, Key Creek plate, EMA,USGS colln. 28587-PC, USNM Nos. 530524, 25 (map 4, no. 174).

Howard Pass quadrangle

11-14. Bispathodus aculeatus plumulus Rhodes, Austin, and Druce, upper and outer lateral views of Pa element, X100, and Pseudopolygnathus nodomarginatus (E.R. Branson), upper and lower views of Pa element, X80, field no. 92AD55-22, from 
limestone and shale member of the Kayak Shale $\sim 12 \mathrm{~m}$ below contact with the Rough Mountain Creek unit; USGS colln. 32450-PC, USNM Nos. 530526, 27.

15. Bispathodus utahensis Sandberg and Gutschick, outer lateral view of Pa element, X80, field no. 91AD6U, Lisburne Group, Rim Butte unit, Ipnavik River allochthon, USGS colln. 31742-PC, USNM No. 530528.

16. Bispathodus aculeatus plumulus Rhodes, Austin, and Druce, nodosus morphotype, $\mathrm{Pa}$ element, upper lateral view, X80, field no. 91ADo70C, Kayak Shale, limestone and shale member, USGS colln. 31748-PC, USNM No. 530529.

17-21. Bispathodus utahensis Sandberg and Gutschick, Sc, Sb, M, and Pb elements, 2 inner lateral, 1 postero-lateral, and 1 inner lateral views, X50, and Gnathodus cuneiformis Mehl and Thomas, juvenile Pa element, upper view, X100, field no. 92AD35AA, Kuna Formation, USGS colln. 32441-PC, USNM Nos. 530530-34.

22-24. Geniculatus claviger Hass, s.f., Pb? elements. Figs. 22 and 23, upper and oblique lower views of nearly complete element, X80, field no. 92ARM37B, Rim Butte unit, Lisburne Group, Ipnavik River allochthon, USGS colln. 32471-PC, USNM No. 530535, and fig. 24, upper view of incomplete element, X75, field no. 91Tr09B, Rim Butte unit, Lisburne Group, USGS colln. Ipnavik River allochthon, USGS colln. 31840-PC, USNM No. 530536.

25. Scaliognathus anchoralis Branson and Mehl, S element bar fragment, lateral view, X100, field no. 92AD35C-15.5 m, type section of Kuna Formation, USGS colln. 32442-PC, USNM No. 530537.

26-28. Protognathodus cordiformis Lane, Sandberg and Ziegler, X100, Pseudopolygnathus marginatus (Branson and Mehl), X80, and Gnathodus 
pseudosemiglaber Thompson and Fellows, Pa elements,X100, upper views, field no. 91Tr13, Rim Butte unit, Lisburne Group, Ipnavik River allochthon, USGS colln. 31841-PC, USNM Nos. 530538-40.

29. Palmatolepis sp. of Famennian morphotype redeposited during middle Osagean or younger Mississippian time, Pa element, upper view, X60, field no. 92AD20-14.5, Rim Butte unit, Ipnavik River allochthon, USGS colln. 32426-PC, USNM No. 530541.

30. Gnathodus pseudosemiglaber Thompson and Fellows, Pa element, upper view, X80, field no. 91Tr13, Rim Butte unit, Ipnavik River allochthon, USGS colln. 31841-PC, USNM No. 530542.

\section{PLATE 4}

Conodonts from the Lisburne Group, Howard Pass quadrangle (scanning electron micrographs; illustrated specimens are reposited in the U.S. National Museum, USNM, Washington, D.C.; see table 15 for conodont age, geologic setting, and location of collections listed below)

Howard Pass quadrangle (continued)

Figures 1-4. Doliognathus latus Branson and Mehl, P elements: figs. 1 and 2, upper views of two poorly preserved, incomplete P elements, X80, field no. 92AD214-18.5, Rim Butte unit, Lisburne Group, Ipnavik River allochthon, USGS colln. 32464-PC, USNM Nos. 530542, 43; figs. 3 and 4, upper and lower views of relatively complete P element, X75, field no. 91Tr09B, Rim Butte unit, Ipnavik River allochthon, USGS colln. 31840-PC, USNM No. 530544. 
5, 6. Mestognathus praebeckmanni Sandberg, Johnston, Orchard, and von Bitter, upper and lower views of subadult Pa element, X100, field no. 92AD214-44, Rim Butte unit, Lisburne Group, Ipnavik River allochthon, USGS colln. 32465-PC, USNM No. 530545.

7. Gnathodus punctatus (Cooper), Pa element, upper view, X80, field no. 92AD50C, late Kinderhookian-very early Osagean species redeposited during middle Osagean or younger Mississippian time, Rim Butte unit, Ipnavik River allochthon, USGS colln. 32447-PC, USNM No. 530546. 